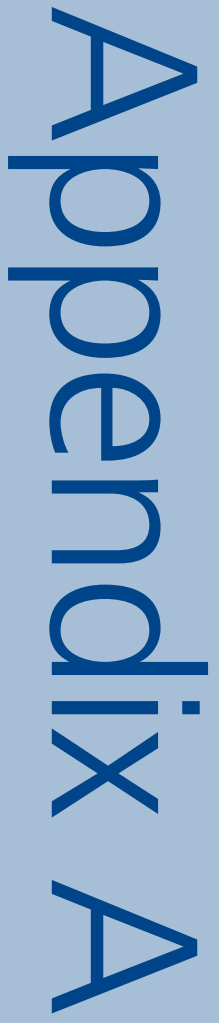




\section{Table A1}

\section{Components of household expenditure, 2008}

based on weighted data and including children's expenditure

\begin{tabular}{|c|c|c|c|c|c|}
\hline & & $\begin{array}{r}\text { rage weekly } \\
\text { expenditure } \\
\text { all house- } \\
\text { holds (f) }\end{array}$ & $\begin{array}{r}\text { Total } \\
\text { weekly } \\
\text { expenditure } \\
(\mathrm{f} \text { million) }\end{array}$ & $\begin{array}{r}\text { Recording } \\
\text { house- } \\
\text { holds } \\
\text { in sample }\end{array}$ & $\begin{array}{r}\text { Percentage } \\
\text { standard } \\
\text { error (full } \\
\text { method) }\end{array}$ \\
\hline \multicolumn{3}{|c|}{ Total number of households } & \multicolumn{3}{|c|}{5,850} \\
\hline \multicolumn{6}{|c|}{ Commodity or service } \\
\hline \multicolumn{2}{|c|}{ Food \& non-alcoholic drinks } & 50.70 & 1,302 & 5,800 & 0.8 \\
\hline 1.1 Food & & 46.70 & 1,198 & 5,800 & 0.8 \\
\hline \multirow{4}{*}{1.1 .1} & Bread, rice and cereals & 4.90 & 125 & 5,680 & 1.0 \\
\hline & 1.1.1.1 Rice & 0.40 & 9 & 1,400 & 6.3 \\
\hline & 1.1.1.2 Bread & 2.60 & 67 & 5,530 & 1.0 \\
\hline & 1.1.1.3 Other breads and cereals & 1.90 & 49 & 4,620 & 1.4 \\
\hline 1.1 .2 & Pasta products & 0.40 & 10 & 2,400 & 2.7 \\
\hline \multirow{3}{*}{1.1 .3} & Buns, cakes, biscuits etc. & 3.20 & 82 & 5,150 & 1.4 \\
\hline & 1.1.3.1 Buns, crispbread and biscuits & 1.80 & 46 & 4,740 & 1.5 \\
\hline & 1.1.3.2 Cakes and puddings & 1.40 & 36 & 3,810 & 2.1 \\
\hline 1.1 .4 & Pastry (savoury) & 0.70 & 17 & 2,000 & 2.5 \\
\hline 1.1.5 & Beef (fresh, chilled or frozen) & 1.60 & 41 & 2,700 & 2.4 \\
\hline 1.1.6 & Pork (fresh, chilled or frozen) & 0.60 & 17 & 1,460 & 3.3 \\
\hline 1.1.7 & Lamb (fresh, chilled or frozen) & 0.60 & 17 & 1,120 & 3.6 \\
\hline 1.1.8 & Poultry (fresh, chilled or frozen) & 1.90 & 49 & 3,030 & 2.0 \\
\hline 1.1 .9 & Bacon and ham & 0.90 & 24 & 2,730 & 2.1 \\
\hline \multirow[t]{5}{*}{ 1.1.10 } & Other meats and meat preparations & 5.20 & 133 & 5,200 & 1.4 \\
\hline & 1.1.10.1 Sausages & 0.70 & 18 & 2,600 & 2.4 \\
\hline & 1.1.10.2 Offal, pate etc. & 0.10 & 3 & 790 & 5.0 \\
\hline & 1.1.10.3 Other preserved or processed meat and meat preparations & 4.30 & 112 & 5,010 & 1.4 \\
\hline & 1.1.10.4 Other fresh, chilled or frozen edible meat & 0.00 & 1 & 40 & 41.7 \\
\hline \multirow[t]{4}{*}{ 1.1.11 } & Fish and fish products & 2.30 & 59 & 3,850 & 2.2 \\
\hline & 1.1.11.1 Fish (fresh, chilled or frozen) & 0.80 & 20 & 1,520 & 3.8 \\
\hline & 1.1.11.2 Seafood, dried, smoked or salted fish & 0.50 & 14 & 1,290 & 4.2 \\
\hline & 1.1.11.3 Other preserved or processed fish and seafood & 1.00 & 26 & 3,010 & 2.6 \\
\hline \multirow[t]{4}{*}{ 1.1.12 } & Milk & 2.60 & 68 & 5,360 & 1.4 \\
\hline & 1.1.12.1 Whole milk & 0.70 & 17 & 1,720 & 3.6 \\
\hline & 1.1.12.2 Low fat milk & 1.80 & 46 & 4,430 & 1.7 \\
\hline & 1.1.12.3 Preserved milk & 0.20 & 4 & 440 & 7.6 \\
\hline 1.1 .13 & Cheese and curd & 1.70 & 43 & 4,180 & 1.6 \\
\hline 1.1.14 & Eggs & 0.60 & 16 & 3,350 & 1.7 \\
\hline \multirow[t]{3}{*}{1.1 .15} & Other milk products & 1.90 & 48 & 4,380 & 1.7 \\
\hline & 1.1.15.1 Other milk products & 0.90 & 22 & 3,330 & 2.1 \\
\hline & 1.1.15.2 Yoghurt & 1.00 & 26 & 3,200 & 2.2 \\
\hline 1.1.16 & Butter & 0.40 & 10 & 1,880 & 2.7 \\
\hline 1.1.17 & Margarine, other vegetable fats and peanut butter & 0.50 & 13 & 2,860 & 2.0 \\
\hline \multirow{3}{*}{ 1.1.18 } & Cooking oils and fats & 0.30 & 8 & 1,380 & 3.7 \\
\hline & 1.1.18.1 Olive oil & 0.10 & 3 & 490 & 6.2 \\
\hline & 1.1.18.2 Edible oils and other edible animal fats & 0.20 & 4 & 990 & 4.6 \\
\hline \multirow[t]{7}{*}{1.1 .19} & Fresh fruit & 3.00 & 76 & 4,920 & 1.6 \\
\hline & 1.1.19.1 Citrus fruits (fresh) & 0.50 & 12 & 2,590 & 2.6 \\
\hline & 1.1.19.2 Bananas (fresh) & 0.50 & 13 & 3,710 & 1.6 \\
\hline & 1.1.19.3 Apples (fresh) & 0.50 & 14 & 2,920 & 2.0 \\
\hline & 1.1.19.4 Pears (fresh) & 0.10 & 4 & 1,180 & 3.5 \\
\hline & 1.1.19.5 Stone fruits (fresh) & 0.40 & 10 & 1,620 & 3.5 \\
\hline & 1.1.19.6 Berries (fresh) & 0.90 & 24 & 2,710 & 2.3 \\
\hline 1.1 .20 & Other fresh, chilled or frozen fruits & 0.40 & 9 & 1,690 & 3.5 \\
\hline 1.1 .21 & Dried fruit and nuts & 0.50 & 12 & 1,860 & 3.4 \\
\hline 1.1 .22 & Preserved fruit and fruit based products & 0.10 & 3 & 1,080 & 4.1 \\
\hline \multirow[t]{6}{*}{ 1.1.23 } & Fresh vegetables & 3.70 & 96 & 5,220 & 1.4 \\
\hline & 1.1.23.1 Leaf and stem vegetables (fresh or chilled) & 0.70 & 19 & 3,440 & 2.0 \\
\hline & 1.1.23.2 Cabbages (fresh or chilled) & 0.40 & 10 & 2,900 & 2.1 \\
\hline & 1.1.23.3 Vegetables grown for their fruit (fresh, chilled or frozen) & 1.30 & 33 & 4,300 & 1.8 \\
\hline & 1.1.23.4 Root crops, non-starchy bulbs and mushrooms & & & & \\
\hline & (fresh, chilled or frozen) & 1.30 & 33 & 4,660 & 1.6 \\
\hline
\end{tabular}

Note: The commodity and service categories are not comparable with those in publications before 2001-02.

The numbering is sequential, it does not use actual COICOP codes.

Please see page xiii for symbols and conventions used in this report. 


\section{Table A1}

\section{Components of household expenditure, 2008 (cont.)}

based on weighted data and including children's expenditure

\begin{tabular}{|c|c|c|c|c|c|c|c|}
\hline & & & & $\begin{array}{r}\text { Average weekly } \\
\text { expenditure } \\
\text { all house- } \\
\text { holds (f) }\end{array}$ & $\begin{array}{r}\text { Total } \\
\text { weekly } \\
\text { expenditure } \\
(\mathrm{f} \text { million) }\end{array}$ & $\begin{array}{r}\text { Recording } \\
\text { house- } \\
\text { holds } \\
\text { in sample }\end{array}$ & $\begin{array}{r}\text { Percentage } \\
\text { standard } \\
\text { error (full } \\
\text { method) }\end{array}$ \\
\hline \multicolumn{8}{|c|}{ Commodity or service } \\
\hline \multirow[t]{23}{*}{1} & \multicolumn{7}{|c|}{ Food \& non-alcoholic drinks (continued) } \\
\hline & & 1.1 .24 & Dried vegetables & 0.00 & 1 & 250 & 56.6 \\
\hline & & 1.1 .25 & Other preserved or processed vegetables & 1.20 & 30 & 4,260 & 0.0 \\
\hline & & 1.1 .26 & Potatoes & 0.90 & 23 & 4,020 & 1.5 \\
\hline & & 1.1 .27 & Other tubers and products of tuber vegetables & 1.30 & 32 & 3,950 & 1.6 \\
\hline & \multirow{3}{*}{\multicolumn{2}{|c|}{1.1 .28}} & Sugar and sugar products & 0.30 & 8 & 2,180 & 2.6 \\
\hline & & & 1.1.28.1 Sugar & 0.20 & 6 & 1,930 & 2.7 \\
\hline & & & 1.1.28.2 Other sugar products & 0.10 & 2 & 510 & 5.6 \\
\hline & & 1.1 .29 & Jams, marmalades & 0.30 & 7 & 1,720 & 3.7 \\
\hline & & 1.1 .30 & Chocolate & 1.50 & 38 & 3,470 & 2.5 \\
\hline & & 1.1 .31 & Confectionery products & 0.60 & 15 & 2,700 & 2.5 \\
\hline & & 1.1 .32 & Edible ices and ice cream & 0.50 & 13 & 1,790 & 2.7 \\
\hline & \multirow{4}{*}{\multicolumn{2}{|c|}{1.1 .33}} & Other food products & 2.30 & 58 & 4,850 & 2.2 \\
\hline & & & 1.1.33.1 Sauces, condiments & 1.10 & 28 & 3,730 & 1.9 \\
\hline & & & 1.1.33.2 Baker's yeast, dessert preparations, soups & 0.80 & 21 & 3,270 & 3.4 \\
\hline & & & 1.1.33.3 Salt, spices, culinary herbs and other food products & 0.40 & 10 & 1,580 & 10.0 \\
\hline & \multirow[t]{7}{*}{1.2} & \multicolumn{2}{|c|}{ Non-alcoholic drinks } & 4.00 & 104 & 5,260 & 1.3 \\
\hline & & 1.2 .1 & Coffee & 0.60 & 15 & 1,770 & 3.1 \\
\hline & & 1.2 .2 & Tea & 0.40 & 11 & 2,050 & 2.3 \\
\hline & & 1.2 .3 & Cocoa and powdered chocolate & 0.10 & 2 & 520 & 6.3 \\
\hline & & 1.2 .4 & Fruit and vegetable juices & 1.10 & 29 & 3,450 & 1.9 \\
\hline & & 1.2 .5 & Mineral or spring waters & 0.20 & 6 & 1,200 & 4.7 \\
\hline & & 1.2 .6 & Soft drinks (inc. fizzy and ready to drink fruit drinks) & 1.60 & 41 & 3,770 & 2.1 \\
\hline \multirow[t]{17}{*}{2} & \multicolumn{3}{|c|}{ Alcoholic drink, tobacco \& narcotics } & 10.80 & 277 & 3,600 & 2.0 \\
\hline & \multirow{6}{*}{\multicolumn{2}{|c|}{2.1 Alcoho }} & ic drinks & 6.20 & 160 & 2,970 & 2.5 \\
\hline & & & Spirits and liqueurs (brought home) & 1.20 & 30 & 820 & 4.1 \\
\hline & & & Wines, fortified wines (brought home) & 3.30 & 84 & 2,030 & 3.5 \\
\hline & & & 2.1.2.1 Wine from grape or other fruit (brought home) & 2.90 & 75 & 1,870 & 3.7 \\
\hline & & & 2.1.2.2 Fortified wine (brought home) & 0.20 & 4 & 210 & 8.9 \\
\hline & & & 2.1.2.3 Champagne and sparkling wines (brought home) & 0.20 & 4 & 170 & 12.1 \\
\hline & \multirow{3}{*}{\multicolumn{2}{|c|}{2.1 .3}} & Beer, lager, ciders and perry (brought home) & 1.70 & 44 & 1,510 & 3.4 \\
\hline & & & 2.1.3.1 Beer and lager (brought home) & 1.50 & 38 & 1,320 & 3.6 \\
\hline & & & 2.1.3.2 Ciders and perry (brought home) & 0.30 & 7 & 390 & 8.0 \\
\hline & & 2.1 .4 & Alcopops (brought home) & 0.10 & 2 & 110 & 16.0 \\
\hline & \multirow[t]{6}{*}{2.2} & \multicolumn{2}{|c|}{ Tobacco and narcotics } & 4.60 & 117 & 1,390 & 3.2 \\
\hline & & 2.2 .1 & Cigarettes & 3.90 & 100 & 1,270 & 3.5 \\
\hline & & \multirow[t]{4}{*}{2.2 .2} & Cigars, other tobacco products and narcotics & 0.70 & 17 & 360 & 7.5 \\
\hline & & & 2.2.2.1 Cigars & 0.10 & 3 & 40 & 20.8 \\
\hline & & & 2.2.2.2 Other tobacco & 0.50 & 13 & 330 & 6.9 \\
\hline & & & 2.2.2.3 Narcotics & {$[0.10]$} & [1] & - & 47.0 \\
\hline 3 & \multicolumn{3}{|c|}{ Clothing \& footwear } & 21.60 & 554 & 3,940 & 2.2 \\
\hline & \multicolumn{3}{|c|}{3.1 Clothing } & 17.50 & 449 & 3,720 & 2.5 \\
\hline & & 3.1 .1 & Men's outer garments & 4.50 & 116 & 1,200 & 5.7 \\
\hline & & 3.1 .2 & Men's under garments & 0.30 & 9 & 440 & 8.1 \\
\hline & & 3.1 .3 & Women's outer garments & 7.60 & 196 & 2,170 & 2.9 \\
\hline & & 3.1.4 & Women's under garments & 1.10 & 27 & 1,110 & 4.9 \\
\hline & & 3.1 .5 & Boys' outer garments (5-15) & 0.80 & 20 & 430 & 7.7 \\
\hline & & 3.1 .6 & Girls' outer garments (5-15) & 1.00 & 25 & 540 & 6.1 \\
\hline & & 3.1.7 & Infants' outer garments (under 5) & 0.60 & 16 & 450 & 7.0 \\
\hline & & 3.1 .8 & Children's under garments (under 16) & 0.30 & 8 & 500 & 6.1 \\
\hline
\end{tabular}

Note: The commodity and service categories are not comparable with those in publications before 2001-02.

The numbering is sequential, it does not use actual COICOP codes.

Please see page xiii for symbols and conventions used in this report. 


\section{Table A1}

\section{Components of household expenditure, 2008 (cont.)}

based on weighted data and including children's expenditure

\begin{tabular}{|c|c|c|c|c|c|c|c|c|}
\hline & & & & & $\begin{array}{r}\text { verage weekly } \\
\text { expenditure } \\
\text { all house- } \\
\text { holds (f) }\end{array}$ & $\begin{array}{r}\text { Total } \\
\text { weekly } \\
\text { expenditure } \\
(\mathrm{f} \text { million) }\end{array}$ & $\begin{array}{r}\text { Recording } \\
\text { house- } \\
\text { holds } \\
\text { in sample }\end{array}$ & $\begin{array}{r}\text { Percentage } \\
\text { standard } \\
\text { error (full } \\
\text { method) }\end{array}$ \\
\hline & modi & ity or se & vice & & & & & \\
\hline 3 & Clot & thing \& & ootwear ( & (continued) & & & & \\
\hline & & 3.1 .9 & Accessor & & 0.70 & 18 & 900 & 5.5 \\
\hline & & & 3.1.9.1 & Men's accessories & 0.20 & 6 & 300 & 8.7 \\
\hline & & & 3.1 .9 .2 & Women's accessories & 0.30 & 8 & 460 & 6.7 \\
\hline & & & 3.1.9.3 & Children's accessories & 0.10 & 3 & 230 & 10.6 \\
\hline & & & 3.1.9.4 & Protective head gear (crash helmets) & {$[0.00]$} & [1] & 20 & 35.2 \\
\hline & & 3.1.10 & Haberda & shery, clothing materials and clothing hire & 0.30 & 7 & 270 & 19.9 \\
\hline & & 3.1.11 & Dry clear & ners, laundry and dyeing & 0.30 & 7 & 200 & 10.4 \\
\hline & & & 3.1.11.1 & Dry cleaners and dyeing & 0.20 & 6 & 160 & 11.3 \\
\hline & & & 3.1.11.2 & Laundry, launderettes & 0.00 & 1 & 40 & 20.2 \\
\hline & 3.2 & Footw & & & 4.10 & 106 & 1,620 & 3.2 \\
\hline & & 3.2 .1 & Footwea & for men & 1.20 & 31 & 420 & 6.2 \\
\hline & & 3.2 .2 & Footwea & r for women & 2.10 & 53 & 950 & 4.4 \\
\hline & & 3.2 .3 & Footwea & r for children (5 to 15 years) and infants (under 5 ) & 0.80 & 21 & 470 & 6.6 \\
\hline & & 3.2 .4 & Repair ar & nd hire of footwear & 0.10 & 1 & 60 & 19.2 \\
\hline 4 & Hou & Ising(ne & )$^{1}$, fuel \& & power & 53.00 & 1,362 & 5,820 & 1.9 \\
\hline & 4.1 & Actual & entals for & housing & 31.60 & 812 & 1,610 & 3.5 \\
\hline & & 4.1.1 & Gross rer & & 31.50 & 810 & 1,610 & 3.5 \\
\hline & & 4.1.2 & less hous & sing benefit, rebates and allowances received & 12.10 & 312 & 1,080 & 3.6 \\
\hline & & 4.1.3 & Net rent & & 19.40 & 498 & 1,220 & 5.1 \\
\hline & & 4.1.4 & Second d & dwelling - rent & {$[0.10]$} & [2] & - & 87.2 \\
\hline & 4.2 & Mainte & hance and & repair of dwelling & 7.20 & 185 & 2,590 & 5.0 \\
\hline & & 4.2 .1 & Central h & heating repairs & 1.50 & 38 & 1,520 & 6.3 \\
\hline & & 4.2 .2 & House $\mathrm{m}$ & laintenance etc. & 3.90 & 100 & 1,150 & 7.0 \\
\hline & & 4.2 .3 & Paint, wa & allpaper, timber & 0.90 & 23 & 440 & 7.6 \\
\hline & & 4.2 .4 & Equipme & nt hire, small materials & 0.90 & 23 & 420 & 17.0 \\
\hline & 4.3 & Water & upply and & miscellaneous services relating to the dwelling & 7.40 & 191 & 4,900 & 1.3 \\
\hline & & 4.3.1 & Water ch & harges & 6.30 & 161 & 4,710 & 0.8 \\
\hline & & 4.3 .2 & Other re & gular housing payments including service charge for rent & 1.10 & 27 & 640 & 8.0 \\
\hline & & 4.3 .3 & Refuse $c c$ & ollection, including skip hire & {$[0.10]$} & [2] & 20 & 34.4 \\
\hline & 4.4 & Electric & ty, gas an & d other fuels & 18.90 & 487 & 5,540 & 1.0 \\
\hline & & 4.4 .1 & Electricit & & 9.00 & 232 & 5,410 & 1.1 \\
\hline & & 4.4 .2 & Gas & & 8.20 & 210 & 4,270 & 1.4 \\
\hline & & 4.4 .3 & Other fu & & 1.70 & 44 & 590 & 7.5 \\
\hline & & & 4.4.3.1 & Coal and coke & 0.20 & 6 & 120 & 20.1 \\
\hline & & & 4.4.3.2 & Oil for central heating & 1.40 & 37 & 480 & 8.0 \\
\hline & & & 4.4.3.3 & Paraffin, wood, peat, hot water etc. & 0.10 & 2 & 50 & 24.9 \\
\hline 5 & Hou & usehold & joods \& se & ervices & 30.10 & 774 & 5,350 & 3.2 \\
\hline & 5.1 & Furnitu & e and fur & nishings, carpets and other floor coverings & 16.60 & 427 & 2,200 & 4.8 \\
\hline & & 5.1 .1 & Furniture & e and furnishings & 13.00 & 333 & 1,820 & 5.4 \\
\hline & & & 5.1.1.1 & Furniture & 11.90 & 304 & 1,250 & 5.7 \\
\hline & & & 5.1 .1 .2 & Fancy, decorative goods & 0.90 & 23 & 740 & 11.1 \\
\hline & & & 5.1 .1 .3 & Garden furniture & 0.20 & 6 & 40 & 29.7 \\
\hline & & 5.1 .2 & Floor cov & verings & 3.70 & 94 & 800 & 6.5 \\
\hline & & & 5.1 .2 .1 & Soft floor coverings & 3.20 & 82 & 750 & 6.6 \\
\hline & & & 5.1 .2 .2 & Hard floor coverings & 0.50 & 12 & 60 & 27.2 \\
\hline & 5.2 & Housel & old textile & & 1.50 & 40 & 1,100 & 5.9 \\
\hline & & 5.2 .1 & Bedroom & n textiles, including duvets and pillows & 0.70 & 18 & 450 & 7.3 \\
\hline & & 5.2 .2 & Other ho & ousehold textiles, including cushions, towels, curtains & 0.80 & 21 & 780 & 8.3 \\
\hline
\end{tabular}

Note: The commodity and service categories are not comparable with those in publications before 2001-02.

The numbering is sequential, it does not use actual COICOP codes.

Please see page xiii for symbols and conventions used in this report.

1 Excluding mortgage interest payments, council tax and NI rates.

2 The figure included in total expenditure is net rent as opposed to gross rent 


\section{Table A1}

\section{Components of household expenditure, 2008 (cont.)}

based on weighted data and including children's expenditure

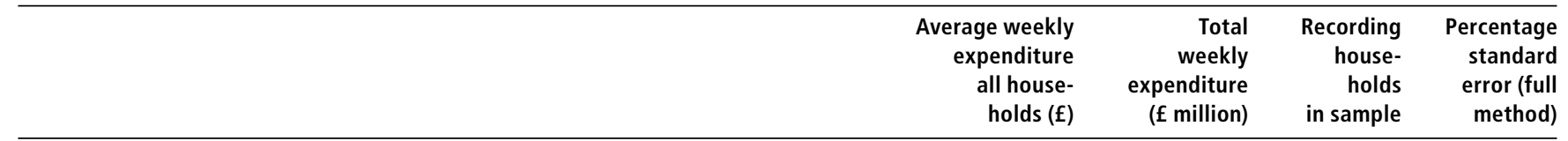

Commodity or service

5 Household goods \& services (continued)

5.3 Household appliances

5.3.1 Gas cookers

3.50
$[0.20]$
0.20
0.70
0.60

1.10
$[0.10]$
0.40
0.20
$[0.00]$
1.30
0.40
0.50
-
0.40
2.10
0.30
0.30
0.40
0.60
0.60
5.10
2.20
1.00
1.10
1.10
0.70
0.20
0.10
0.10
1.80
1.30
0.50
$[0.00]$

90

[4]

5.3.2 Electric cookers, combined gas/electric cookers

5.3.3 Clothes washing machines and drying machines

5.3.4 Refrigerators, freezers and fridge-freezers

5.3.5 Other major electrical appliances, dishwashers, micro-waves vacuum cleaners, heaters etc.

5.3.6 Fire extinguisher, water softener, safes etc

5.3.7 Small electric household appliances, excluding hairdryers

5.3.8 Repairs to gas and electrical appliances and spare parts

5.3.9 Rental/hire of major household appliances

5.4 Glassware, tableware and household utensils

5.4.1 Glassware, china, pottery, cutlery and silverware

5.4.2 Kitchen and domestic utensils

5.4.3 Repair of glassware, tableware and household utensils

5.4.4 Storage and other durable household articles

5.5 Tools and equipment for house and garden

5.5.1 Electrical tools

5.5.2 Garden tools, equipment and accessories e.g. lawn mowers etc.

5.5.3 Small tools

5.5.4 Door, electrical and other fittings

5.5.5 Electrical consumables

5.6 Goods and services for routine household maintenance

5.6.1 Cleaning materials

5.6.1.1 Detergents, washing-up liquid, washing powder

5.6.1.2 Disinfectants, polishes, other cleaning materials etc.

5.6.2 Household goods and hardware

5.6.2.1 Kitchen disposables

5.6.2.2 Household hardware and appliances, matches

5.6.2.3 Kitchen gloves, cloths etc.

5.6.2.4 Pins, needles, tape measures, nails, nuts and bolts etc.

5.6.3 Domestic services, carpet cleaning, hire/repair of furniture/furnishings

5.6.3.1 Domestic services, including cleaners, gardeners, au pairs

5.6.3.2 Carpet cleaning, ironing service, window cleaner

5.6.3.3 Hire/repair of household furniture and furnishings

$490 \quad 11.9$

$-\quad 41.7$

$40 \quad 47.0$

$90 \quad 19.6$

$60 \quad 29.0$

$150 \quad 19.4$

$-\quad 78.1$

$190 \quad 13.9$

$50 \quad 23.3$

50
$-\quad 43.2$

$1,660 \quad 4.5$

$640 \quad 7.5$

$950 \quad 5.9$

540

1,880

$70 \quad 25.4$

280

$380 \quad 10.7$

$450 \quad 12.1$

$1,210 \quad 4.9$

$4,890 \quad 2.8$

$\begin{array}{ll}4,090 & 1.7\end{array}$

$2,840 \quad 2.2$

$3,330 \quad 2.1$

$3,600 \quad 2.3$

$3,050 \quad 2.6$

$680 \quad 6.2$

$950 \quad 4.7$

$370 \quad 7.2$

$910 \quad 7.1$

$360 \quad 9.1$

$640 \quad 8.3$

$-\quad 58.1$

6 Health

6.1 Medical products, appliances and equipment

6.1.1 Medicines, prescriptions and healthcare products

5.10

6.1.1.1 NHS prescription charges and payments

3.10

1.70

0.30

6.1.1.2 Medicines and medical goods (not NHS)

1.20

6.1.1.3 Other medical products (e.g. plasters, condoms, hot water bottle etc.)

6.1.1.4 Non-optical appliances and equipment (e.g. wheelchairs, batteries for hearing aids, shoe build-up)

6.1.2 Spectacles, lenses, accessories and repairs

6.1.2.1 Purchase of spectacles, lenses, prescription sunglasses

6.1.2.2 Accessories/repairs to spectacles/lenses 0.00

6.2 Hospital services 2.00

$\begin{array}{lll}\text { 6.2.1 Out patient services } & 2.00\end{array}$

6.2.1.1 NHS medical, optical, dental and medical auxiliary services $\quad 0.90$

6.2.1.2 Private medical, optical, dental and medical auxiliary services 1.10

6.2.1.3 Other services

6.2.2 In-patient hospital services

Note: The commodity and service categories are not comparable with those in publications before 2001-02. The numbering is sequential, it does not use actual COICOP codes.

Please see page xiii for symbols and conventions used in this report. 


\section{Table A1}

\section{Components of household expenditure, 2008 (cont.)}

based on weighted data and including children's expenditure

\begin{tabular}{|c|c|c|c|c|c|c|c|c|}
\hline & & & & $\begin{array}{r}\text { Avera } \\
\text { e) }\end{array}$ & $\begin{array}{l}\text { age weekly } \\
\text { expenditure } \\
\text { all house- } \\
\text { holds (f) }\end{array}$ & $\begin{array}{r}\text { Total } \\
\text { weekly } \\
\text { expenditure } \\
(\mathrm{f} \text { million) }\end{array}$ & $\begin{array}{r}\text { Recording } \\
\text { house- } \\
\text { holds } \\
\text { in sample }\end{array}$ & $\begin{array}{r}\text { Percentage } \\
\text { standard } \\
\text { error (full } \\
\text { method) }\end{array}$ \\
\hline \multicolumn{9}{|c|}{ Commodity or service } \\
\hline \multirow[t]{50}{*}{7} & \multicolumn{4}{|c|}{ Transport } & 63.40 & 1,628 & 5,000 & 2.0 \\
\hline & \multirow{4}{*}{\multicolumn{2}{|c|}{$\begin{array}{ll}7.1 & \text { Purch } \\
7.1 .1\end{array}$}} & \multirow{2}{*}{\multicolumn{2}{|c|}{$\begin{array}{l}\text { of vehicles } \\
\text { Purchase of new cars and vans }\end{array}$}} & 21.10 & 543 & 1,420 & 4.2 \\
\hline & & & & & 6.60 & 171 & 350 & 7.5 \\
\hline & & & 7.1.1.1 & Outright purchases & 4.20 & 109 & 130 & 10.1 \\
\hline & & & 7.1.1.2 & Loan/Hire Purchase of new car/van & 2.40 & 62 & 230 & 9.5 \\
\hline & \multirow{3}{*}{\multicolumn{2}{|c|}{ 7.1.2 }} & \multicolumn{2}{|c|}{ Purchase of second hand cars or vans } & 13.60 & 350 & 1,080 & 5.1 \\
\hline & & & 7.1.2.1 & Outright purchases & 9.80 & 253 & 640 & 6.7 \\
\hline & & & 7.1.2.2 & Loan/Hire Purchase of second hand car/van & 3.80 & 97 & 500 & 5.7 \\
\hline & & 7.1.3 & \multicolumn{2}{|c|}{ Purchase of motorcycles } & 0.90 & 23 & 80 & 20.5 \\
\hline & & & 7.1.3.1 & Outright purchases of new or second hand motorcycles & 0.40 & 9 & 30 & 23.6 \\
\hline & & & 7.1.3.2 & Loan/Hire Purchase of new or second hand motorcycles & 0.20 & 4 & 20 & 29.5 \\
\hline & & & 7.1.3.3 & Purchase of bicycles and other vehicles & 0.40 & 10 & 30 & 40.5 \\
\hline & & \multicolumn{3}{|c|}{ Operation of personal transport } & 31.80 & 816 & 4,300 & 1.8 \\
\hline & & 7.2 .1 & \multicolumn{2}{|c|}{ Spares and accessories } & 2.40 & 61 & 480 & 11.3 \\
\hline & & & 7.2.1.1 & Car/van accessories and fittings & 0.60 & 15 & 130 & 33.8 \\
\hline & & & 7.2.1.2 & Car/van spare parts & 1.50 & 39 & 270 & 10.9 \\
\hline & & & 7.2.1.3 & Motorcycle accessories and spare parts & {$[0.00]$} & [1] & 20 & 49.0 \\
\hline & & & 7.2.1.4 & Bicycle accessories, repairs and other costs & 0.20 & 5 & 100 & 17.2 \\
\hline & & 7.2 .2 & \multicolumn{2}{|c|}{ Petrol, diesel and other motor oils } & 21.00 & 540 & 3,810 & 1.7 \\
\hline & & & 7.2.2.1 & Petrol & 15.70 & 404 & 3,200 & 1.9 \\
\hline & & & 7.2.2.2 & Diesel oil & 5.30 & 135 & 1,070 & 3.9 \\
\hline & & & 7.2.2.3 & Other motor oils & 0.10 & 2 & 70 & 16.1 \\
\hline & & 7.2 .3 & Repairs & and servicing & 6.20 & 160 & 1,800 & 3.6 \\
\hline & & & 7.2.3.1 & Car or van repairs, servicing and other work & 6.20 & 159 & 1,800 & 3.6 \\
\hline & & & 7.2.3.2 & Motorcycle repairs and servicing & 0.00 & 1 & 20 & 30.2 \\
\hline & & 7.2 .4 & Other $r$ & lotoring costs & 2.10 & 55 & 2,170 & 4.2 \\
\hline & & & 7.2.4.1 & Motoring organisation subscription (e.g. AA and RAC) & 0.40 & 9 & 910 & 5.8 \\
\hline & & & 7.2.4.2 & Garage rent, other costs (excluding fines), car washing etc. & 0.60 & 14 & 310 & 8.5 \\
\hline & & & 7.2.4.3 & Parking fees, tolls, and permits (excluding motoring fines) & 0.80 & 21 & 1,260 & 6.4 \\
\hline & & & 7.2.4.4 & Driving lessons & 0.30 & 8 & 60 & 15.9 \\
\hline & & & 7.2.4.5 & Anti-freeze, battery water, cleaning materials & 0.10 & 3 & 290 & 9.7 \\
\hline & \multirow{4}{*}{\multicolumn{2}{|c|}{$\begin{array}{ll}7.3 & \text { Transp } \\
& 7.3 .1\end{array}$}} & rt servic & & 10.50 & 269 & 2,390 & 4.1 \\
\hline & & & \multicolumn{2}{|c|}{ Rail and tube fares } & 2.40 & 62 & 730 & 5.8 \\
\hline & & & 7.3.1.1 & Season tickets & 1.00 & 25 & 150 & 10.4 \\
\hline & & & 7.3.1.2 & Other than season tickets & 1.50 & 37 & 630 & 6.1 \\
\hline & & 7.3.2 & \multicolumn{2}{|c|}{ Bus and coach fares } & 1.40 & 35 & 1,000 & 5.0 \\
\hline & & & 7.3.2.1 & Season tickets & 0.50 & 12 & 200 & 8.0 \\
\hline & & & \multirow{2}{*}{\multicolumn{2}{|c|}{$\begin{array}{l}\text { 7.3.2.2 Other than season tickets } \\
\text { Combined fares }\end{array}$}} & 0.90 & 23 & 900 & 5.5 \\
\hline & & 7.3.3 & & & 1.20 & 31 & 230 & 9.3 \\
\hline & & & 7.3.3.1 & Combined fares other than season tickets & 0.30 & 8 & 130 & 13.0 \\
\hline & & & \multirow{2}{*}{\multicolumn{2}{|c|}{$\begin{array}{l}\text { 7.3.3.2 Combined fares season tickets } \\
\text { Other travel and transport }\end{array}$}} & 0.90 & 23 & 120 & 11.9 \\
\hline & & 7.3.4 & & & 5.40 & 140 & 1,350 & 7.0 \\
\hline & & & 7.3.4.1 & Air fares (within UK) & 0.30 & 9 & 20 & 37.1 \\
\hline & & & 7.3.4.2 & Air fares (international) & 1.50 & 39 & 50 & 19.7 \\
\hline & & & 7.3.4.3 & School travel & 0.10 & 2 & 40 & 48.8 \\
\hline & & & 7.3.4.4 & Taxis and hired cars with drivers & 1.10 & 29 & 800 & 5.2 \\
\hline & & & 7.3.4.5 & Other personal travel and transport services & 0.20 & 6 & 290 & 11.7 \\
\hline & & & 7.3.4.6 & Hire of self-drive cars, vans, bicycles & 0.20 & 6 & 30 & 27.3 \\
\hline & & & 7.3.4.7 & Car leasing & 1.80 & 46 & 220 & 8.1 \\
\hline & & & 7.3.4.8 & Water travel, ferries and season tickets & 0.10 & 3 & 60 & 28.3 \\
\hline
\end{tabular}

Note: The commodity and service categories are not comparable with those in publications before 2001-02. The numbering is sequential, it does not use actual COICOP codes.

Please see page xiii for symbols and conventions used in this report. 


\section{Table A1}

\section{Components of household expenditure, 2008 (cont.)}

based on weighted data and including children's expenditure

\begin{tabular}{|c|c|c|c|c|c|c|c|c|}
\hline & & & & $\begin{array}{r}\text { Averag } \\
\text { exp } \\
a\end{array}$ & $\begin{array}{l}\text { ge weekly } \\
\text { xpenditure } \\
\text { all house- } \\
\text { holds (f) }\end{array}$ & $\begin{array}{r}\text { Total } \\
\text { weekly } \\
\text { expenditure } \\
(\mathrm{f} \text { million })\end{array}$ & $\begin{array}{r}\text { Recording } \\
\text { house- } \\
\text { holds } \\
\text { in sample }\end{array}$ & $\begin{array}{r}\text { Percentage } \\
\text { standard } \\
\text { error (full } \\
\text { method) }\end{array}$ \\
\hline \multicolumn{9}{|c|}{ Commodity or service } \\
\hline \multirow[t]{11}{*}{8} & \multicolumn{4}{|c|}{ Communication } & 12.00 & 307 & 5,570 & 1.2 \\
\hline & \multirow{5}{*}{$\begin{array}{ll}8.1 & F \\
8.2 & 1 \\
& \varepsilon \\
& \varepsilon \\
& \varepsilon \\
& \varepsilon\end{array}$} & \multirow{2}{*}{\multicolumn{3}{|c|}{$\begin{array}{l}\text { Postal services } \\
\text { Telephone and telefax equipment }\end{array}$}} & 0.50 & 12 & 1,050 & 5.9 \\
\hline & & & & & 0.50 & 13 & 160 & 11.4 \\
\hline & & 8.2 .1 & \multicolumn{2}{|c|}{ Telephone purchase } & 0.10 & 2 & 30 & 25.8 \\
\hline & & 8.2 .2 & \multicolumn{2}{|c|}{ Mobile phone purchase } & 0.40 & 10 & 130 & 12.7 \\
\hline & & 8.2 .3 & \multicolumn{2}{|c|}{ Answering machine, fax machine, modem purchase } & {$[0.00]$} & [0] & - & 66.9 \\
\hline & \multirow[t]{5}{*}{8.3} & Telepl & ne and & elefax services & 11.00 & 282 & 5,530 & 1.1 \\
\hline & & 8.3 .1 & \multicolumn{2}{|c|}{ Telephone account } & 5.70 & 146 & 5,150 & 1.1 \\
\hline & & 8.3 .2 & \multicolumn{2}{|c|}{ Telephone coin and other payments } & 0.00 & 1 & 70 & 18.3 \\
\hline & & 8.3 .3 & \multicolumn{2}{|c|}{ Mobile phone account } & 4.00 & 104 & 2,120 & 2.5 \\
\hline & & 8.3 .4 & \multicolumn{2}{|c|}{ Mobile phone - other payments } & 1.20 & 31 & 920 & 3.9 \\
\hline \multirow[t]{44}{*}{9} & \multicolumn{4}{|c|}{ Recreation \& culture } & 60.10 & 1,543 & 5,790 & 3.6 \\
\hline & \multirow{4}{*}{\multicolumn{2}{|c|}{$\begin{array}{cl}9.1 & \text { Audio } \\
& 9.1 .1\end{array}$}} & isual, ph & otographic and information processing equipment & 7.20 & 184 & 1,720 & 7.2 \\
\hline & & & Audio & quipment and accessories, CD players & 1.30 & 33 & 750 & 7.8 \\
\hline & & & 9.1.1.1 & Audio equipment, CD players including in car & 0.50 & 13 & 130 & 16.7 \\
\hline & & & 9.1 .1 .2 & Audio accessories e.g. tapes, headphones etc. & 0.80 & 20 & 650 & 6.9 \\
\hline & & 9.1 .2 & TV, vid & o and computers & 4.80 & 123 & 1,210 & 8.7 \\
\hline & & & 9.1.2.1 & Purchase of TV and digital decoder & 1.70 & 43 & 140 & 18.4 \\
\hline & & & 9.1 .2 .2 & Satellite dish purchase and installation & {$[0.00]$} & [0] & - & 81.7 \\
\hline & & & 9.1 .2 .3 & Cable TV connection & {$[0.00]$} & [0] & - & 78.0 \\
\hline & & & 9.1 .2 .4 & Video recorder & {$[0.00]$} & [0] & - & 78.0 \\
\hline & & & 9.1 .2 .5 & DVD player/recorder & 0.20 & 5 & 30 & 26.2 \\
\hline & & & 9.1 .2 .6 & Blank, pre-recorded video cassettes, DVDs & 1.00 & 26 & 770 & 6.0 \\
\hline & & & 9.1 .2 .7 & Personal computers, printers and calculators & 1.70 & 43 & 370 & 13.5 \\
\hline & & & 9.1 .2 .8 & Spare parts for TV, video, audio & 0.20 & 4 & 70 & 25.5 \\
\hline & & & 9.1 .2 .9 & Repair of audio-visual, photographic and information & & & & \\
\hline & & & & processing & 0.10 & 2 & 30 & 23.6 \\
\hline & & 9.1 .3 & Photog & aphic, cine and optical equipment & 1.10 & 27 & 150 & 25.0 \\
\hline & & & 9.1 .3 .1 & Photographic and cine equipment & 1.00 & 26 & 100 & 26.1 \\
\hline & & & 9.1 .3 .2 & Camera films & 0.00 & 1 & 30 & 24.4 \\
\hline & & & 9.1.3.3 & Optical instruments, binoculars, telescopes, microscopes & {$[0.00]$} & [1] & 10 & 44.7 \\
\hline & 9.2 & Other & najor dur & ables for recreation and culture & 4.10 & 106 & 130 & 40.1 \\
\hline & & 9.2 .1 & Purchas & e of boats, trailers and horses & {$[2.50]$} & [65] & 10 & 62.1 \\
\hline & & 9.2 .2 & Purchas & e of caravans, mobile homes (including decoration) & 0.50 & 13 & 20 & 47.0 \\
\hline & & 9.2 .3 & Accessc & ries for boats, horses, caravans and motor caravans & 0.20 & 6 & 20 & 44.5 \\
\hline & & 9.2 .4 & Musica & instruments (purchase and hire) & 0.10 & 3 & 40 & 37.8 \\
\hline & & 9.2 .5 & Major c & urables for indoor recreation & [0.00] & [0] & - & 118.5 \\
\hline & & 9.2 .6 & Mainte & nance and repair of other major durables & 0.40 & 10 & 40 & 34.0 \\
\hline & & 9.2.7 & Purchas & e of motor caravan (new and second-hand) - outright purchas & se $[0.30]$ & [9] & - & 45.4 \\
\hline & & 9.2 .8 & Purchas & e of motor caravan (new and second-hand) - loan/HP & {$[0.00]$} & [1] & - & 67.4 \\
\hline & 9.3 & Other & ecreatior & al items and equipment, gardens and pets & 10.70 & 276 & 3,970 & 2.9 \\
\hline & & 9.3.1 & Games, & toys and hobbies & 1.80 & 47 & 1,450 & 4.5 \\
\hline & & 9.3 .2 & Compu & er software and games & 2.00 & 51 & 410 & 8.1 \\
\hline & & & 9.3.2.1 & Computer software and game cartridges & 1.10 & 28 & 350 & 7.2 \\
\hline & & & 9.3.2.2 & Computer games consoles & 0.90 & 23 & 100 & 15.1 \\
\hline & & 9.3 .3 & Equipm & ent for sport, camping and open-air recreation & 0.90 & 23 & 460 & 11.1 \\
\hline & & 9.3 .4 & Horticu & Itural goods, garden equipment and plants etc. & 2.60 & 67 & 2,080 & 4.8 \\
\hline & & & 9.3.4.1 & $\mathrm{BBQ}$ and swings & 0.00 & 1 & 30 & 39.4 \\
\hline & & & 9.3.4.2 & Plants, flowers, seeds, fertilisers, insecticides & 2.50 & 64 & 2,020 & 5.0 \\
\hline & & & 9.3 .4 .3 & Garden decorative & 0.10 & 2 & 80 & 18.4 \\
\hline & & & 9.3.4.4 & Artificial flowers, pot pourri & 0.00 & 1 & 80 & 21.3 \\
\hline & & 9.3 .5 & Pets an & pet food & 3.40 & 87 & 2,120 & 4.9 \\
\hline & & & 9.3.5.1 & Pet food & 1.80 & 47 & 1,990 & 3.3 \\
\hline & & & 9.3.5.2 & Pet purchase and accessories & 0.60 & 15 & 570 & 12.0 \\
\hline & & & 9.3.5.3 & Veterinary and other services for pets identified separately & 1.00 & 25 & 190 & 11.7 \\
\hline
\end{tabular}

Note: The commodity and service categories are not comparable with those in publications before 2001-02.

The numbering is sequential, it does not use actual COICOP codes.

Please see page xiii for symbols and conventions used in this report. 


\section{Table A1}

\section{Components of household expenditure, 2008 (cont.)}

based on weighted data and including children's expenditure

\begin{tabular}{|c|c|c|c|c|c|c|c|c|}
\hline & & & & $\begin{array}{r}\text { Average } \\
\text { expe } \\
\text { al } \\
h\end{array}$ & $\begin{array}{l}\text { e weekly } \\
\text { enditure } \\
\text { IIl house- } \\
\text { holds (f) }\end{array}$ & $\begin{array}{r}\text { Total } \\
\text { weekly } \\
\text { expenditure } \\
(\mathrm{f} \text { million) }\end{array}$ & $\begin{array}{r}\text { Recording } \\
\text { house- } \\
\text { holds } \\
\text { in sample }\end{array}$ & $\begin{array}{r}\text { Percentage } \\
\text { standard } \\
\text { error (full } \\
\text { method) }\end{array}$ \\
\hline \multicolumn{9}{|c|}{ Commodity or service } \\
\hline \multirow[t]{40}{*}{9} & \multicolumn{8}{|c|}{ Recreation \& culture (continued) } \\
\hline & \multirow{7}{*}{\multicolumn{2}{|c|}{$\begin{array}{ll}9.4 & \text { Recrea } \\
9.4 .1\end{array}$}} & ional anc & cultural services & 17.30 & 443 & 5,510 & 2.0 \\
\hline & & & Sports & dmissions, subscriptions, leisure class fees and equipment hire & 4.80 & 123 & 2,080 & 3.9 \\
\hline & & & 9.4.1.1 & Spectator sports: admission charges & 0.50 & 12 & 180 & 14.9 \\
\hline & & & 9.4 .1 .2 & Participant sports (excluding subscriptions) & 1.20 & 30 & 940 & 6.2 \\
\hline & & & 9.4 .1 .3 & Subscriptions to sports and social clubs & 1.50 & 39 & 860 & 6.8 \\
\hline & & & 9.4 .1 .4 & Leisure class fees & 1.60 & 41 & 860 & 5.7 \\
\hline & & & 9.4.1.5 & Hire of equipment for sport and open air recreation & 0.00 & 1 & 20 & 44.6 \\
\hline & \multirow{4}{*}{\multicolumn{2}{|c|}{9.4 .2}} & Cinema & theatre and museums etc. & 1.90 & 50 & 960 & 5.6 \\
\hline & & & 9.4.2.1 & Cinemas & 0.50 & 14 & 520 & 5.1 \\
\hline & & & 9.4.2.2 & Live entertainment: theatre, concerts, shows & 1.10 & 28 & 330 & 9.2 \\
\hline & & & 9.4 .2 .3 & $\begin{array}{l}\text { Museums, zoological gardens, theme parks, houses and } \\
\text { gardens }\end{array}$ & 0.30 & 8 & 210 & 9.2 \\
\hline & \multirow{8}{*}{\multicolumn{2}{|c|}{9.4 .3}} & TV, vide & o, satellite rental, cable subscriptions, TV licences and Internet & 5.80 & 150 & 5,150 & 1.3 \\
\hline & & & 9.4.3.1 & TV licences & 2.20 & 57 & 4,930 & 0.4 \\
\hline & & & 9.4.3.2 & Satellite subscriptions & 2.40 & 63 & 1,760 & 2.6 \\
\hline & & & 9.4.3.3 & Rent for TV/Satellite/VCR & 0.20 & 6 & 190 & 10.1 \\
\hline & & & 9.4.3.4 & Cable subscriptions & 0.50 & 14 & 620 & 6.0 \\
\hline & & & 9.4.3.5 & TV slot meter payments & {$[0.00]$} & [1] & 10 & 37.4 \\
\hline & & & 9.4.3.6 & Video, cassette and CD hire & 0.10 & 2 & 140 & 11.0 \\
\hline & & & 9.4.3.7 & Internet subscription fees & 0.30 & 8 & 310 & 10.0 \\
\hline & \multirow{4}{*}{\multicolumn{2}{|c|}{9.4 .4}} & Miscella & neous entertainments & 1.20 & 31 & 1,110 & 7.6 \\
\hline & & & 9.4 .4 .1 & Admissions to clubs, dances, discos, bingo & 0.60 & 14 & 500 & 8.5 \\
\hline & & & 9.4 .4 .2 & Social events and gatherings & 0.40 & 10 & 270 & 19.2 \\
\hline & & & 9.4.4.3 & Subscriptions for leisure activities and other subscriptions & 0.30 & 7 & 520 & 8.6 \\
\hline & & \multirow[t]{2}{*}{9.4 .5} & Develop & ment of film, deposit for film development, passport & & & & \\
\hline & & & photos, & holiday and school photos & 0.30 & 8 & 250 & 18.2 \\
\hline & & \multirow[t]{5}{*}{9.4 .6} & Gamblit & g payments & 3.20 & 81 & 2,770 & 5.9 \\
\hline & & & 9.4.6.1 & Football pools stakes & 0.10 & 2 & 50 & 26.5 \\
\hline & & & 9.4 .6 .2 & Bingo stakes excluding admission & 0.30 & 8 & 160 & 12.1 \\
\hline & & & 9.4 .6 .3 & Lottery & 1.90 & 49 & 2,480 & 3.0 \\
\hline & & & 9.4.6.4 & Bookmaker, tote, other betting stakes & 0.90 & 23 & 630 & 18.3 \\
\hline & 9.5 & \multicolumn{3}{|c|}{ Newspapers, books and stationery } & 6.00 & 155 & 5,090 & 1.9 \\
\hline & & 9.5 .1 & Books & & 1.40 & 35 & 1,250 & 4.5 \\
\hline & & 9.5 .2 & Station & ry, diaries, address books, art materials & 0.70 & 18 & 1,480 & 6.6 \\
\hline & & 9.5 .3 & Cards, c & alendars, posters and other printed matter & 1.20 & 31 & 2,670 & 2.9 \\
\hline & & 9.5 .4 & Newspa & pers & 1.90 & 48 & 3,670 & 2.2 \\
\hline & & 9.5 .5 & Magazi & hes and periodicals & 0.90 & 23 & 2,670 & 2.8 \\
\hline & 9.6 & Packag & holiday & & 14.70 & 378 & 880 & 5.5 \\
\hline & & 9.6 .1 & Packag & holidays - UK & 1.10 & 28 & 180 & 9.4 \\
\hline & & 9.6 .2 & Packag & holidays - abroad & 13.60 & 350 & 730 & 5.9 \\
\hline \multirow[t]{13}{*}{10} & \multicolumn{4}{|c|}{ Education } & 6.20 & 159 & 470 & 12.8 \\
\hline & \multicolumn{4}{|c|}{ 10.1 Education fees } & 5.90 & 151 & 340 & 13.4 \\
\hline & & 10.1.1 & Nursery & and primary education & 1.00 & 25 & 60 & 17.6 \\
\hline & & 10.1.2 & Second & ry education & 1.30 & 35 & 50 & 27.6 \\
\hline & & 10.1.3 & Sixth fo & rm college/college education & 0.40 & 11 & 40 & 41.5 \\
\hline & & 10.1.4 & Univers & ty education & 2.00 & 52 & 130 & 13.4 \\
\hline & & 10.1.5 & Other e & ducation & 1.10 & 29 & 90 & 41.9 \\
\hline & \multicolumn{4}{|c|}{ 10.2 Payments for school trips, other ad-hoc expenditure } & 0.30 & 8 & 140 & 16.0 \\
\hline & & 10.2 .1 & Nursery & and primary education & 0.10 & 2 & 80 & 18.1 \\
\hline & & 10.2 .2 & Second & ry education & 0.10 & 3 & 50 & 28.6 \\
\hline & & 10.2 .3 & Sixth fo & m college/college education & {$[0.00]$} & [0] & - & 75.3 \\
\hline & & 10.2 .4 & Univers & ty education & {$[0.00]$} & [1] & - & 49.3 \\
\hline & & 10.2 .5 & Other $\mathrm{e}$ & ducation & {$[0.00]$} & [1] & 10 & 33.7 \\
\hline
\end{tabular}

Note: The commodity and service categories are not comparable with those in publications before 2001-02.

The numbering is sequential, it does not use actual COICOP codes.

Please see page xiii for symbols and conventions used in this report. 


\section{Table A1}

\section{Components of household expenditure, 2008 (cont.)}

based on weighted data and including children's expenditure

\begin{tabular}{|c|c|c|c|c|c|c|}
\hline & & $\begin{array}{r}\text { Avera } \\
\text { e }\end{array}$ & $\begin{array}{r}\text { rage weekly } \\
\text { expenditure } \\
\text { all house- } \\
\text { holds (f) }\end{array}$ & $\begin{array}{r}\text { Total } \\
\text { weekly } \\
\text { expenditure } \\
(\mathrm{f} \text { million) }\end{array}$ & $\begin{array}{r}\text { Recording } \\
\text { house- } \\
\text { holds } \\
\text { in sample }\end{array}$ & $\begin{array}{r}\text { Percentage } \\
\text { standard } \\
\text { error (full } \\
\text { method) }\end{array}$ \\
\hline \multicolumn{7}{|c|}{ Commodity or service } \\
\hline \multirow[t]{18}{*}{11} & \multicolumn{2}{|c|}{ Restaurants \& hotels } & 37.70 & 969 & 5,090 & 2.1 \\
\hline & \multicolumn{2}{|c|}{ 11.1 Catering services } & 31.00 & 796 & 5,050 & 2.0 \\
\hline & 11.1.1 & Restaurant and café meals & 13.20 & 340 & 4,100 & 2.4 \\
\hline & 11.1.2 & Alcoholic drinks (away from home) & 7.20 & 185 & 2,550 & 3.0 \\
\hline & 11.1.3 & Take away meals eaten at home & 3.90 & 99 & 2,510 & 2.7 \\
\hline & \multirow[t]{5}{*}{ 11.1.4 } & Other take-away and snack food & 4.20 & 107 & 3,340 & 2.2 \\
\hline & & 11.1.4.1 Hot and cold food & 2.90 & 75 & 2,900 & 2.5 \\
\hline & & 11.1.4.2 Confectionery & 0.30 & 9 & 1,720 & 3.3 \\
\hline & & 11.1.4.3 Ice cream & 0.10 & 3 & 540 & 6.6 \\
\hline & & 11.1.4.4 Soft drinks & 0.80 & 21 & 2,220 & 2.5 \\
\hline & 11.1.5 & Contract catering (food) & 0.60 & 16 & 30 & 30.0 \\
\hline & \multirow[t]{3}{*}{ 11.1.6 } & Canteens & 1.90 & 49 & 1,740 & 3.5 \\
\hline & & 11.1.6.1 School meals & 0.60 & 15 & 610 & 6.1 \\
\hline & & 11.1.6.2 Meals bought and eaten at the workplace & 1.30 & 34 & 1,360 & 4.1 \\
\hline & \multicolumn{2}{|c|}{ 11.2 Accommodation services } & 6.80 & 173 & 1,020 & 5.3 \\
\hline & 11.2.1 & Holiday in the UK & 2.80 & 71 & 650 & 6.1 \\
\hline & 11.2 .2 & Holiday abroad & 4.00 & 102 & 440 & 7.7 \\
\hline & 11.2 .3 & Room hire & {$[0.00]$} & [1] & - & 37.8 \\
\hline \multirow[t]{27}{*}{12} & \multicolumn{2}{|c|}{ Miscellaneous goods and services } & 35.60 & 915 & 5,710 & 2.5 \\
\hline & \multicolumn{2}{|c|}{ 12.1 Personal care } & 9.90 & 254 & 5,030 & 1.9 \\
\hline & 12.1.1 & Hairdressing, beauty treatment & 3.10 & 79 & 1,430 & 3.7 \\
\hline & 12.1.2 & Toilet paper & 0.80 & 20 & 2,720 & 2.2 \\
\hline & \multirow[t]{6}{*}{12.1 .3} & Toiletries and soap & 2.00 & 52 & 3,980 & 1.9 \\
\hline & & 12.1.3.1 Toiletries (disposable including tampons, & & & & \\
\hline & & lipsyl, toothpaste etc.) & 1.20 & 30 & 3,350 & 2.2 \\
\hline & & 12.1.3.2 Bar of soap, liquid soap, shower gel etc. & 0.40 & 9 & 1,690 & 3.7 \\
\hline & & 12.1.3.3 Toilet requisites (durable including razors, & & & & \\
\hline & & hairbrushes, toothbrushes etc.) & 0.50 & 13 & 1,370 & 4.3 \\
\hline & 12.1.4 & Baby toiletries and accessories (disposable) & 0.60 & 16 & 920 & 5.0 \\
\hline & \multirow[t]{4}{*}{ 12.1.5 } & Hair products, cosmetics and electrical appliances for personal care & 3.40 & 87 & 3,140 & 3.1 \\
\hline & & 12.1.5.1 Hair products & 0.80 & 19 & 1,850 & 3.4 \\
\hline & & 12.1.5.2 Cosmetics and related accessories & 2.40 & 62 & 2,320 & 3.7 \\
\hline & & $\begin{array}{l}\text { 12.1.5.3 Electrical appliances for personal care, including } \\
\text { hairdryers, shavers etc. }\end{array}$ & 0.20 & 6 & 110 & 14.4 \\
\hline & \multicolumn{2}{|c|}{ 12.2 Personal effects } & 3.30 & 85 & 1,500 & 13.7 \\
\hline & 12.2 .1 & Jewellery, clocks and watches and other personal effects & 2.10 & 54 & 1,040 & 20.3 \\
\hline & 12.2 .2 & Leather and travel goods (excluding baby items) & 0.80 & 21 & 550 & 8.1 \\
\hline & 12.2 .3 & Sunglasses (non-prescription) & 0.10 & 2 & 90 & 16.9 \\
\hline & 12.2 .4 & Baby equipment (excluding prams and pushchairs) & 0.10 & 4 & 60 & 21.9 \\
\hline & 12.2 .5 & Prams, pram accessories and pushchairs & 0.10 & 3 & 20 & 39.3 \\
\hline & 12.2 .6 & Repairs to personal goods & 0.00 & 1 & 30 & 25.2 \\
\hline & \multicolumn{2}{|c|}{12.3 Social protection } & 3.60 & 93 & 280 & 12.1 \\
\hline & 12.3 .1 & Residential homes & [0.20] & [5] & - & 63.8 \\
\hline & 12.3 .2 & Home help & 0.30 & 7 & 40 & 33.2 \\
\hline & 12.3 .3 & Nursery, crèche, playschools & 0.90 & 23 & 90 & 19.7 \\
\hline & 12.3 .4 & Child care payments & 2.30 & 58 & 170 & 15.2 \\
\hline
\end{tabular}

Note: The commodity and service categories are not comparable with those in publications before 2001-02. The numbering is sequential, it does not use actual COICOP codes. Please see page xiii for symbols and conventions used in this report. 


\section{Table A1}

\section{Components of household expenditure, 2008 (cont.)}

based on weighted data and including children's expenditure

\begin{tabular}{|c|c|c|c|c|c|c|c|}
\hline & & & & $\begin{array}{r}\text { erage weekly } \\
\text { expenditure } \\
\text { all house- } \\
\text { holds (f) }\end{array}$ & $\begin{array}{r}\text { Total } \\
\text { weekly } \\
\text { expenditure } \\
(\mathrm{f} \text { million) }\end{array}$ & $\begin{array}{r}\text { Recording } \\
\text { house- } \\
\text { holds } \\
\text { in sample }\end{array}$ & $\begin{array}{r}\text { Percentage } \\
\text { standard } \\
\text { error (full } \\
\text { method) }\end{array}$ \\
\hline \multicolumn{8}{|c|}{ Commodity or service } \\
\hline \multicolumn{8}{|c|}{12 Miscellaneous goods and services (continued) } \\
\hline \multirow[t]{10}{*}{12.4} & \multicolumn{3}{|c|}{ Insurance } & 14.60 & 374 & 5,210 & 1.5 \\
\hline & \multirow[t]{4}{*}{12.4 .1} & Household insurances & old insurances & 5.00 & 129 & 4,690 & 1.4 \\
\hline & & 12.4.1.1 & Structure insurance & 2.50 & 63 & 3,770 & 1.8 \\
\hline & & 12.4.1.2 & Contents insurance & 2.50 & 64 & 4,520 & 1.6 \\
\hline & & 12.4.1.3 & Insurance for household appliances & 0.10 & 2 & 90 & 23.2 \\
\hline & 12.4 .2 & Medical i & insurance premiums & 1.40 & 36 & 650 & 6.4 \\
\hline & \multirow[t]{3}{*}{12.4 .3} & Vehicle ir & insurance including boat insurance & 7.90 & 204 & 4,350 & 1.8 \\
\hline & & 12.4.3.1 & Vehicle insurance & 7.90 & 203 & 4,350 & 1.8 \\
\hline & & 12.4.3.2 & Boat insurance (not home) & {$[0.00]$} & [1] & - & 84.5 \\
\hline & 12.4 .4 & Non-pacl & ckage holiday, other travel insurance & 0.20 & 4 & 50 & 25.7 \\
\hline \multirow[t]{18}{*}{12.5} & \multicolumn{3}{|c|}{ Other services } & 4.20 & 109 & 1,890 & 7.9 \\
\hline & \multirow[t]{6}{*}{12.5 .1} & \multicolumn{2}{|c|}{ Moving house } & 2.10 & 53 & 350 & 8.3 \\
\hline & & 12.5.1.1 & Moving and storage of furniture & 0.30 & 7 & 150 & 18.5 \\
\hline & & 12.5.1.2 & Property transaction - purchase and sale & 0.90 & 24 & 100 & 13.8 \\
\hline & & 12.5.1.3 & Property transaction - sale only & 0.40 & 10 & 50 & 18.9 \\
\hline & & 12.5.1.4 & Property transaction - purchase only & 0.30 & 8 & 80 & 14.9 \\
\hline & & 12.5.1.5 & Property transaction - other payments & 0.20 & 5 & 80 & 19.6 \\
\hline & \multirow[t]{5}{*}{12.5 .2} & Bank, bu & uilding society, post office, credit card charges & 0.40 & 11 & 890 & 6.1 \\
\hline & & 12.5.2.1 & Bank and building society charges & 0.40 & 10 & 760 & 6.4 \\
\hline & & 12.5 .2 .2 & Bank and Post Office counter charges & {$[0.00]$} & [0] & 20 & 38.1 \\
\hline & & 12.5.2.3 & Annual standing charge for credit cards & 0.00 & 1 & 150 & 16.1 \\
\hline & & 12.5.2.4 & Commission travellers' cheques and currency & {$[0.00]$} & [0] & - & 60.2 \\
\hline & \multirow[t]{6}{*}{12.5 .3} & \multicolumn{2}{|c|}{ Other services and professional fees } & 1.70 & 45 & 1,010 & 16.4 \\
\hline & & 12.5.3.1 & Other professional fees including court fines & 0.60 & 15 & 60 & 23.9 \\
\hline & & 12.5.3.2 & Legal fees & {$[0.10]$} & [3] & 20 & 41.7 \\
\hline & & 12.5.3.3 & Funeral expenses & [0.30] & [8] & - & 75.6 \\
\hline & & 12.5.3.4 & TU and professional organisations & 0.60 & 15 & 810 & 11.7 \\
\hline & & 12.5.3.5 & Other payments for services e.g. photocopying & 0.10 & 4 & 180 & 17.7 \\
\hline \multicolumn{4}{|c|}{ 1-12 All expenditure groups } & 386.30 & 9,924 & 5,850 & 1.2 \\
\hline \multirow[t]{13}{*}{13} & \multicolumn{3}{|c|}{ Other expenditure items } & 84.60 & 2,174 & 5,470 & 2.6 \\
\hline & \multirow[t]{5}{*}{13.1} & Housing: & : mortgage interest payments, council tax etc. & 58.40 & 1,500 & 5,060 & 1.7 \\
\hline & & 13.1.1 & Mortgage interest payments & 37.50 & 963 & 2,230 & 2.3 \\
\hline & & 13.1.2 & Mortgage protection premiums & 1.90 & 48 & 1,110 & 4.2 \\
\hline & & 13.1.3 & Council tax, domestic rates & 18.50 & 475 & 5,020 & 0.8 \\
\hline & & 13.1.5 & Council tax, mortgage (second dwelling) & 0.50 & 14 & 30 & 29.5 \\
\hline & \multirow[t]{4}{*}{13.2} & Licences, & , fines and transfers & 3.20 & 83 & 4,260 & 2.4 \\
\hline & & 13.2 .1 & Stamp duty, licences and fines (excluding motoring fines) & 0.30 & 9 & 90 & 18.4 \\
\hline & & 13.2 .2 & Motoring fines & {$[0.10]$} & [2] & 20 & 27.8 \\
\hline & & 13.2 .3 & Motor vehicle road taxation payments less refunds & 2.80 & 72 & 4,250 & 1.3 \\
\hline & \multirow[t]{3}{*}{13.3} & Holiday s & spending & 11.50 & 295 & 330 & 15.6 \\
\hline & & 13.3 .1 & Money spent abroad & 11.50 & 294 & 330 & 15.6 \\
\hline & & 13.3 .2 & Duty free goods bought in UK & {$[0.00]$} & [0] & - & 65.1 \\
\hline
\end{tabular}

Note: The commodity and service categories are not comparable with those in publications before 2001-02.

The numbering is sequential, it does not use actual COICOP codes.

Please see page xiii for symbols and conventions used in this report. 


\section{Table A1}

\section{Components of household expenditure, 2008 (cont.)}

based on weighted data and including children's expenditure

\begin{tabular}{|c|c|c|c|c|c|c|}
\hline & & & $\begin{array}{r}\text { Average weekly } \\
\text { expenditure } \\
\text { all house- } \\
\text { holds (f) }\end{array}$ & $\begin{array}{r}\text { Total } \\
\text { weekly } \\
\text { expenditure } \\
(\mathrm{f} \text { million) }\end{array}$ & $\begin{array}{l}\text { Recording } \\
\text { house- } \\
\text { holds } \\
\text { in sample }\end{array}$ & $\begin{array}{r}\text { Percentage } \\
\text { standard } \\
\text { error (full } \\
\text { method) }\end{array}$ \\
\hline \multicolumn{7}{|c|}{ Commodity or service } \\
\hline \multirow{2}{*}{$\begin{array}{l}13 \\
13.4\end{array}$} & \multicolumn{6}{|c|}{ Other expenditure items (continued) } \\
\hline & \multicolumn{2}{|c|}{ Money transfers and credit } & 11.50 & 297 & 3,070 & 6.0 \\
\hline & \multirow[t]{3}{*}{13.4 .1} & Money, cash gifts given to children & 0.10 & 2 & 110 & 14.2 \\
\hline & & 13.4.1.1 Money given to children for specific purposes & 0.10 & 2 & 110 & 14.2 \\
\hline & & 13.4.1.2 Cash gifts to children (no specific purpose) & {$[0.00]$} & [0] & - & 82.8 \\
\hline & \multirow[t]{5}{*}{13.4 .2} & Cash gifts and donations & 9.80 & 251 & 2,570 & 6.9 \\
\hline & & 13.4.2.1 Money/presents given to those outside the household & 3.40 & 88 & 1,040 & 9.4 \\
\hline & & 13.4.2.2 Charitable donations and subscriptions & 2.30 & 60 & 1,710 & 7.6 \\
\hline & & 13.4.2.3 Money sent abroad & 2.00 & 51 & 350 & 23.5 \\
\hline & & 13.4.2.4 Maintenance allowance expenditure & 2.00 & 53 & 150 & 14.5 \\
\hline & \multirow[t]{3}{*}{13.4 .3} & Club instalment payments (child) and interest on credit cards & 1.70 & 43 & 980 & 5.8 \\
\hline & & 13.4.3.1 Club instalment payment & {$[0.00]$} & {$[0]$} & - & \\
\hline & & 13.4.3.2 Interest on credit cards & 1.70 & 43 & 980 & 5.8 \\
\hline \multicolumn{3}{|c|}{ Total expenditure } & 471.00 & 12,098 & 5,850 & 1.3 \\
\hline \multirow{15}{*}{$\begin{array}{l}\text { Othe } \\
14.1\end{array}$} & \multirow{2}{*}{\multicolumn{2}{|c|}{$\begin{array}{l}\text { r items recorded } \\
\text { Life assurance, contributions to pension funds }\end{array}$}} & & & & \\
\hline & & & Life assurance, contributions to pension funds & 522 & 3,040 & 3.3 \\
\hline & 14.1.1 & Life assurance premiums eg mortgage endowment policies & 4.60 & 118 & 1,970 & 6.2 \\
\hline & 14.1.2 & Contributions to pension and superannuation funds etc. & 11.20 & 288 & 1,750 & 2.9 \\
\hline & 14.1.3 & Personal pensions & 4.50 & 116 & 730 & 9.5 \\
\hline & \multicolumn{2}{|c|}{ Other insurance including friendly societies } & 1.20 & 31 & 1,230 & 5.2 \\
\hline & \multicolumn{2}{|c|}{ Income tax, payments less refunds } & 98.60 & 2,532 & 4,630 & 6.5 \\
\hline & 14.3.1 & Income tax paid by employees under PAYE & 72.30 & 1,858 & 3,180 & 2.2 \\
\hline & 14.3.2 & Income tax paid direct eg by retired or unoccupied persons & 2.10 & 53 & 170 & 24.1 \\
\hline & 14.3 .3 & Income tax paid direct by self-employed & 6.70 & 172 & 300 & 11.3 \\
\hline & \multirow[t]{2}{*}{ 14.3.4 } & Income tax deducted at source from income under covenant from & & & & \\
\hline & & investments or from annuities and pensions & 11.60 & 297 & 2,760 & 15.9 \\
\hline & 14.3.5 & Income tax on bonus earnings & 6.90 & 176 & 900 & 59.1 \\
\hline & 14.3 .6 & Income tax refunds under PAYE & 0.30 & 7 & 50 & 30.7 \\
\hline & 14.3 .7 & Income tax refunds other than PAYE & 0.70 & 18 & 300 & 14.5 \\
\hline \multirow[t]{3}{*}{14.4} & \multicolumn{2}{|c|}{ National insurance contribution } & 28.50 & 731 & 3,100 & 1.5 \\
\hline & 14.4.1 & NI contributions paid by employees & 28.40 & 730 & 3,080 & 1.5 \\
\hline & 14.4 .2 & $\mathrm{NI}$ contributions paid by non-employees & 0.10 & 1 & 40 & 25.8 \\
\hline \multirow[t]{9}{*}{14.5} & \multicolumn{2}{|r|}{ Purchase or alteration of dwellings (contracted out), mortgages } & 44.10 & 1,132 & 2,490 & 5.9 \\
\hline & 14.5 .1 & Outright purchase of houses, flats etc. including deposits & {$[0.10]$} & [3] & - & 44.7 \\
\hline & 14.5 .2 & Capital repayment of mortgage & 17.80 & 457 & 1,830 & 2.5 \\
\hline & 14.5 .3 & Central heating installation & 1.20 & 30 & 130 & 20.9 \\
\hline & 14.5 .4 & DIY improvements: Double glazing, kitchen units, sheds etc. & 1.60 & 40 & 90 & 33.7 \\
\hline & 14.5 .5 & Home improvements - contracted out & 18.10 & 464 & 940 & 8.5 \\
\hline & 14.5 .6 & Bathroom fittings & 0.50 & 12 & 100 & 25.6 \\
\hline & 14.5 .7 & Purchase of materials for Capital Improvements & 0.80 & 22 & 50 & 38.2 \\
\hline & 14.5 .8 & Purchase of second dwelling & 4.10 & 105 & 50 & 40.7 \\
\hline \multirow[t]{4}{*}{14.6} & \multicolumn{2}{|c|}{ Savings and investments } & 6.50 & 168 & 910 & 7.5 \\
\hline & 14.6.1 & Savings, investments (excluding AVCs) & 5.70 & 146 & 680 & 8.3 \\
\hline & 14.6 .2 & Additional Voluntary Contributions & 0.70 & 17 & 110 & 14.9 \\
\hline & 14.6 .3 & Food stamps, other food related expenditure & 0.20 & 4 & 190 & 12.5 \\
\hline 14.7 & Pay off & f loan to clear other debt & 2.20 & 56 & 280 & 7.4 \\
\hline 14.8 & Windfa & all receipts from gambling etc. ${ }^{3}$ & 1.70 & 43 & 560 & 13.5 \\
\hline
\end{tabular}

Note: The commodity and service categories are not comparable with those in publications before 2001-02.

The numbering is sequential, it does not use actual COICOP codes.

Please see page xiii for symbols and conventions used in this report.

3 Expressed as an income figure as opposed to an expenditure figure. 


\section{Table A2}

Expenditure on alcoholic drink by place of purchase, 2008

based on weighted data and including children's expenditure

$\begin{array}{rrr}\begin{array}{r}\text { Average weekly } \\ \text { expenditure }\end{array} & \begin{array}{r}\text { Total } \\ \text { weekly }\end{array} & \begin{array}{r}\text { Recording } \\ \text { households } \\ \text { in sample }\end{array} \\ \text { all households } & \begin{array}{r}\text { expenditure } \\ (f)\end{array} & \text { million) }\end{array}$

By type of premises

11 Bought and consumed on licenced premises:

11.1.2 Alcoholic drinks (away from home) $\quad 7.20-185$

$\begin{array}{llll}\text { 11.1.2.2 } & \text { Wine from grape or other fruit (away from home) } & 1.30 & 33\end{array}$

$\begin{array}{llll}11.1 .2 .3 & \text { Fortified wine (away from home) } & 0.00 & 60\end{array}$

$\begin{array}{llll}\text { 11.1.2.4 Champagne and sparkling wines (away from home) } & 0.10 & 3\end{array}$

$\begin{array}{llll}11.1 .2 .5 & \text { Ciders and perry (away from home) } & 0.30 & 6\end{array}$

$\begin{array}{llll}11.1 .2 .6 & \text { Beer and lager (away from home) } & 3.90 & 100\end{array}$

$\begin{array}{lllr}11.1 .2 .7 & \text { Alcopops (away from home) } & 0.10 & 3\end{array}$

$\begin{array}{llll}\text { 11.1.2.8 } & \text { Round of drinks (away from home) } & 0.70 & 18\end{array}$

2 Bought at off-licences (including large supermarket chains):

2.1 Alcoholic drinks

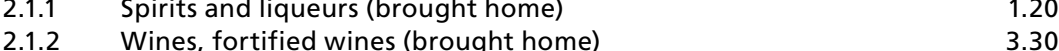

3.30

$\begin{array}{lll}2.1 .2 .1 & \text { Wine from grape or other fruit (brought home) } & 2.90 \\ 2.1 .2 .2 & \text { Fortified } & 0.20\end{array}$

2.1.2.3 Champagne and sparkling wines (brought home) $\quad 0.20$

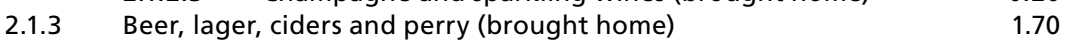

2.1.3.1 Beer and lager (brought home) 1.50

$\begin{array}{ll}\text { 2.1.3.2 Ciders and perry (brought home) } & 0.30\end{array}$

2.1.4 Alcopops (brought home)

2A Bought from large supermarket chains:

2.1A Alcoholic drinks

2.1.1A Spirits and liqueurs (brought home)

2.1.2A Wines, fortified wines (brought home)

2B Bought from other off-licence outlets:

2.1B Alcoholic drinks

2.1.1B Spirits and liqueurs (brought home)

2.1.2B Wines, fortified wines (brought home)

Note: The commodity and service categories are not comparable with those in publications before $2001-02$.

The numbering is sequential, it does not use actual COICOP codes.

Please see page xiii for symbols and conventions used in this report. 


\section{Table A3}

\section{Expenditure on food and non-alcoholic drinks by place of purchase, 2008}

based on weighted data and including children's expenditure

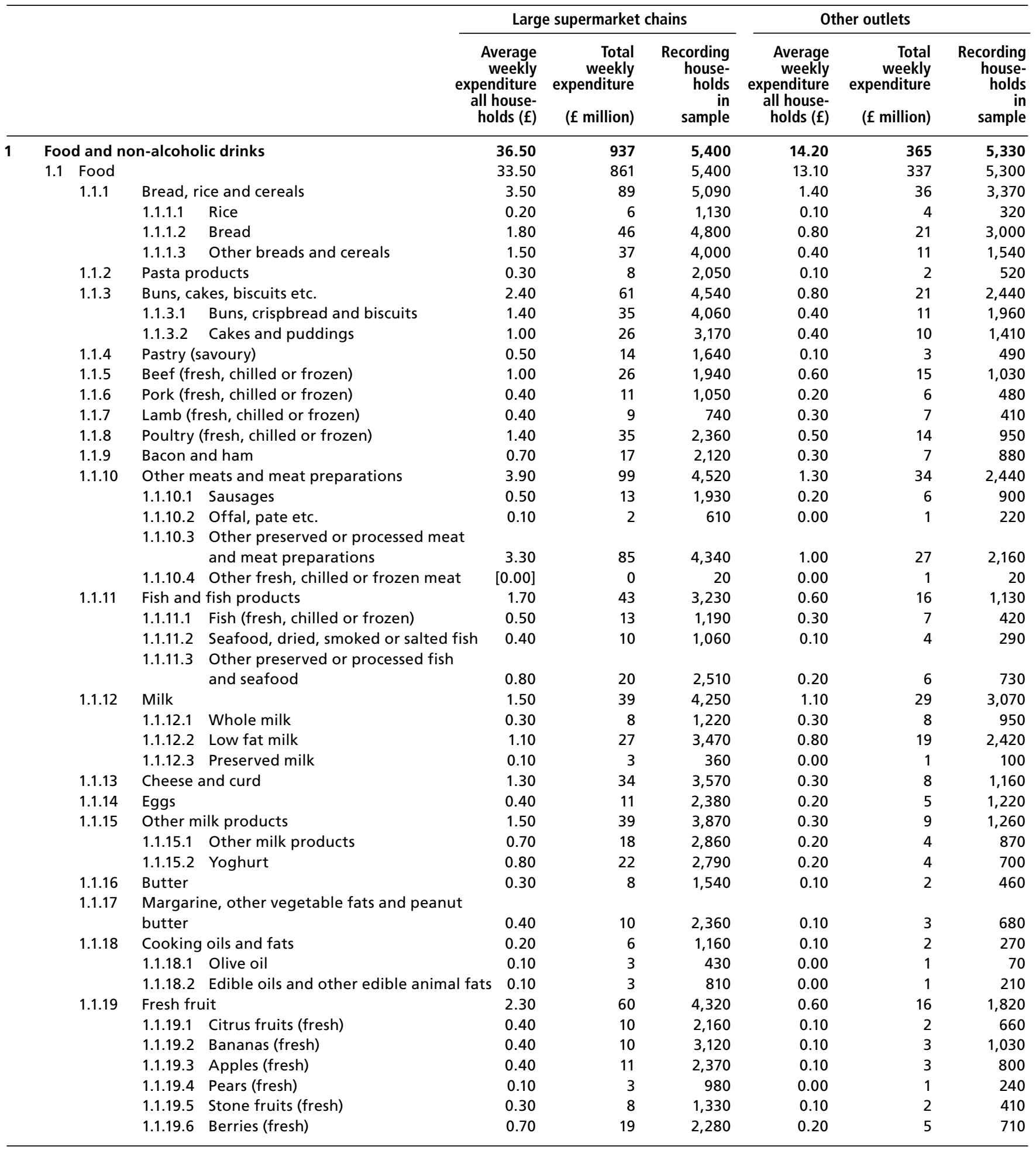

Note: The commodity and service categories are not comparable with those in publications before 2001-02.

The numbering is sequential, it does not use actual COICOP codes.

Please see page xiii for symbols and conventions used in this report. 


\section{Table A3}

\section{Expenditure on food and non-alcoholic drinks by place of purchase, 2008 (cont.)}

based on weighted data and including children's expenditure

\begin{tabular}{|c|c|c|c|c|c|c|c|c|c|}
\hline & & & & \multicolumn{3}{|c|}{ Large supermarket chains } & \multicolumn{3}{|c|}{ Other outlets } \\
\hline & & & $\begin{array}{r}\text { Av } \\
w \\
\text { expen } \\
\text { all } h \\
\text { hol }\end{array}$ & $\begin{array}{l}\text { erage } \\
\text { eekly } \\
\text { diture } \\
\text { ouse- } \\
\text { ds (f) }\end{array}$ & $\begin{array}{r}\text { Total } \\
\text { weekly } \\
\text { expenditure } \\
(£ \text { million })\end{array}$ & $\begin{array}{r}\text { Recording } \\
\text { house- } \\
\text { holds } \\
\text { in } \\
\text { sample }\end{array}$ & $\begin{array}{r}\text { Average } \\
\text { weekly } \\
\text { expenditure } \\
\text { all house- } \\
\text { holds (f) }\end{array}$ & $\begin{array}{r}\text { Total } \\
\text { weekly } \\
\text { expenditure } \\
(\mathrm{f} \text { million })\end{array}$ & $\begin{array}{r}\text { Recording } \\
\text { house- } \\
\text { holds } \\
\text { in } \\
\text { sample }\end{array}$ \\
\hline \multirow[t]{31}{*}{1} & Food & and no & -alcoholic drinks (continued) & & & & & & \\
\hline & & 1.1 .20 & Other fresh, chilled or frozen fruits & 0.30 & 7 & 1,400 & 0.10 & 2 & 380 \\
\hline & & 1.1 .21 & Dried fruit and nuts & 0.30 & 9 & 1,510 & 0.20 & 4 & 510 \\
\hline & & 1.1.22 & Preserved fruit and fruit based products & 0.10 & 3 & 910 & 0.00 & 1 & 200 \\
\hline & & 1.1 .23 & Fresh vegetables & 2.90 & 74 & 4,630 & 0.80 & 21 & 2,200 \\
\hline & & & 1.1.23.1 Leaf and stem vegetables (fresh & & & & & & \\
\hline & & & or chilled) & 0.60 & 15 & 2,990 & 0.10 & 3 & 870 \\
\hline & & & 1.1.23.2 Cabbages (fresh or chilled) & 0.30 & 8 & 2,380 & 0.10 & 3 & 800 \\
\hline & & & 1.1.23.3 Vegetables grown for their fruit (fresh, & & & & & & 0 \\
\hline & & & chilled or frozen) & 1.00 & 27 & 3,710 & 0.30 & 7 & 1,290 \\
\hline & & 1.1.24 & Dried vegetables and other preserved and & & & & & & \\
\hline & & & processed vegetables & 0.60 & 14 & 3,130 & 0.60 & 17 & 2,850 \\
\hline & & 1.1.25 & Potatoes & 0.70 & 17 & 3,180 & 0.20 & 6 & 1,350 \\
\hline & & 1.1 .26 & Other tubers and products of tuber vegetables & 0.90 & 24 & 3,290 & 0.30 & 8 & 1,470 \\
\hline & & 1.1.27 & Sugar and sugar products & 0.20 & 6 & 1,710 & 0.10 & 2 & 600 \\
\hline & & & 1.1.28.1 Sugar & 0.20 & 4 & 1,500 & 0.10 & 2 & 540 \\
\hline & & & 1.1.28.2 Other sugar products & 0.10 & 1 & 420 & 0.00 & 0 & 100 \\
\hline & & 1.1 .28 & Jams, marmalades & 0.20 & 5 & 1,390 & 0.10 & 2 & 410 \\
\hline & & 1.1.29 & Chocolate & 0.90 & 23 & 2,610 & 0.60 & 15 & 1,810 \\
\hline & & 1.1 .30 & Confectionery products & 0.30 & 8 & 1,840 & 0.30 & 7 & 1,490 \\
\hline & & 1.1 .31 & Edible ices and ice cream & 0.40 & 9 & 1,350 & 0.10 & 3 & 580 \\
\hline & & 1.1 .32 & Other food products & 1.60 & 42 & 4,200 & 0.60 & 16 & 1,710 \\
\hline & & & 1.1.32.1 Sauces, condiments & 0.90 & 23 & 3,240 & 0.20 & 5 & 960 \\
\hline & & & $\begin{array}{l}\text { 1.1.32.2 Baker's yeast, dessert preparations, } \\
\text { soups }\end{array}$ & 0.60 & 15 & 2,730 & 0.20 & 5 & 910 \\
\hline & 1.2 & Non-al & oholic drinks & 3.00 & 76 & 4,560 & 1.10 & 28 & 2,700 \\
\hline & & 1.2 .1 & Coffee & 0.40 & 11 & 1,400 & 0.10 & 3 & 460 \\
\hline & & 1.2 .2 & Tea & 0.30 & 8 & 1,530 & 0.10 & 3 & 620 \\
\hline & & 1.2 .3 & Cocoa and powdered chocolate & 0.10 & 2 & 410 & 0.00 & 1 & 120 \\
\hline & & 1.2 .4 & Fruit and vegetable juices (inc. fruit squash) & 0.90 & 24 & 2,980 & 0.20 & 6 & 930 \\
\hline & & 1.2 .5 & Mineral or spring waters & 0.20 & 4 & 940 & 0.10 & 1 & 360 \\
\hline & & 1.2 .6 & Soft drinks & 1.10 & 28 & 3,000 & 0.50 & 14 & 1,820 \\
\hline
\end{tabular}

Note: The commodity and service categories are not comparable with those in publications before 2001-02.

The numbering is sequential, it does not use actual COICOP codes.

Please see page xiii for symbols and conventions used in this report. 


\section{Table A4}

\section{Expenditure on selected items by place of purchase, 2008}

based on weighted data and including children's expenditure

\begin{tabular}{|c|c|c|c|c|c|c|c|c|c|c|}
\hline & & & & & \multicolumn{3}{|c|}{ Large supermarket chains } & \multicolumn{3}{|c|}{ Other outlets } \\
\hline & & & & $\begin{array}{r}\text { Av } \\
v \\
\text { expen } \\
\text { all } \\
\text { ho }\end{array}$ & $\begin{array}{l}\text { lverage } \\
\text { weekly } \\
\text { nditure } \\
\text { house- } \\
\text { olds (f) }\end{array}$ & $\begin{array}{r}\text { Total } \\
\text { weekly } \\
\text { expenditure } \\
(\mathbf{f} \text { million })\end{array}$ & $\begin{array}{r}\text { Recording } \\
\text { house- } \\
\text { holds } \\
\text { in } \\
\text { sample }\end{array}$ & $\begin{array}{r}\text { Average } \\
\text { weekly } \\
\text { expenditure } \\
\text { all house- } \\
\text { holds }(\mathrm{f})\end{array}$ & $\begin{array}{r}\text { Total } \\
\text { weekly } \\
\text { expenditure } \\
(£ \text { million })\end{array}$ & $\begin{array}{r}\begin{array}{r}\text { Recording } \\
\text { house- } \\
\text { holds } \\
\text { in } \\
\text { sample }\end{array}\end{array}$ \\
\hline \multirow[t]{5}{*}{2} & \multicolumn{10}{|c|}{ Alcoholic drink and tobacco } \\
\hline & \multirow{4}{*}{2.2} & 2.2 .1 & \multicolumn{2}{|c|}{ Cigarettes } & 1.00 & 27 & 580 & 2.90 & 73 & 1,110 \\
\hline & & 2.2 .2 & \multicolumn{2}{|c|}{ Cigars and other tobacco products } & 0.20 & 5 & 140 & 0.40 & 11 & 280 \\
\hline & & & 2.2 .2 .1 & Cigars & 0.10 & 1 & 20 & 0.00 & 1 & 20 \\
\hline & & & 2.2 .2 .2 & Other tobacco & 0.10 & 3 & 130 & 0.40 & 10 & 260 \\
\hline 5 & \multicolumn{10}{|c|}{ Household goods and services } \\
\hline & & 5.5 .5 & Electrical & I consumable & 0.20 & 4 & 520 & 0.40 & 11 & 790 \\
\hline \multirow[t]{5}{*}{7} & \multicolumn{10}{|c|}{ Transport } \\
\hline & & 7.2 .2 & \multicolumn{2}{|c|}{ Petrol, diesel \& other motor oils } & 8.60 & 221 & 2,110 & 12.40 & 319 & 2,640 \\
\hline & & & 7.2.2.1 & Petrol & 6.50 & 167 & 1,750 & 9.20 & 237 & 2,160 \\
\hline & & & 7.2.2.2 & Diesel oil & 2.10 & 54 & 480 & 3.10 & 81 & 730 \\
\hline & & & 7.2.2.3 & Other motor oils & {$[0.00]$} & 0 & 20 & 0.10 & 1 & 60 \\
\hline \multirow[t]{2}{*}{8} & \multicolumn{10}{|c|}{ Communication } \\
\hline & 8.1 & Postal & ervices & & - & - & 0 & 0.50 & 12 & 1,050 \\
\hline \multirow[t]{5}{*}{9} & \multicolumn{10}{|c|}{ Recreation and culture } \\
\hline & & & 9.3.5.1 & Pet food & 0.80 & 22 & 1,360 & 1.00 & 25 & 1,090 \\
\hline & & 9.5 .3 & \multicolumn{2}{|c|}{$\begin{array}{l}\text { Cards, calendars, posters and other } \\
\text { printed matter }\end{array}$} & 0.50 & 12 & 1,410 & 1.40 & 37 & 2,560 \\
\hline & & 9.5 .4 & \multicolumn{2}{|c|}{ Newspapers } & 0.30 & 7 & 1,710 & 1.60 & 40 & 3,260 \\
\hline & & 9.5 .5 & \multicolumn{2}{|c|}{ Magazines and periodicals } & 0.30 & 7 & 1,280 & 0.60 & 16 & 1,930 \\
\hline \multirow[t]{6}{*}{12} & \multicolumn{10}{|c|}{ Miscellaneous goods and services } \\
\hline & \multirow{5}{*}{\multicolumn{2}{|c|}{12.1 .2}} & \multicolumn{2}{|c|}{ Toilet paper } & 0.60 & 15 & 2,060 & 0.20 & 5 & 800 \\
\hline & & & $\begin{array}{l}12.1 .3 .1 \\
\&\end{array}$ & $\begin{array}{l}\text { Toiletries and other toilet requisites - } \\
\text { toothpaste, deodorant, tampons, }\end{array}$ & & & & & & \\
\hline & & & 12.1.3.3 & razors, hairbrushes, toothbrushes & 0.90 & 24 & 2,620 & 0.80 & 19 & 1,860 \\
\hline & & & 12.1.3.2 & Bar of soap, liquid soap, shower gel etc & c 0.20 & 5 & 1,190 & 0.10 & 4 & 620 \\
\hline & & & 12.1.5.2 & Cosmetics and related accessories & 0.50 & 13 & 1,210 & 1.90 & 50 & 1,500 \\
\hline
\end{tabular}

Note: The commodity and service categories are not comparable with those in publications before 2001-02.

The numbering is sequential, it does not use actual COICOP codes.

Please see page xiii for symbols and conventions used in this report. 


\section{Table A5}

\section{Expenditure on clothing and footwear by place of purchase, 2008}

based on weighted data and including children's expenditure

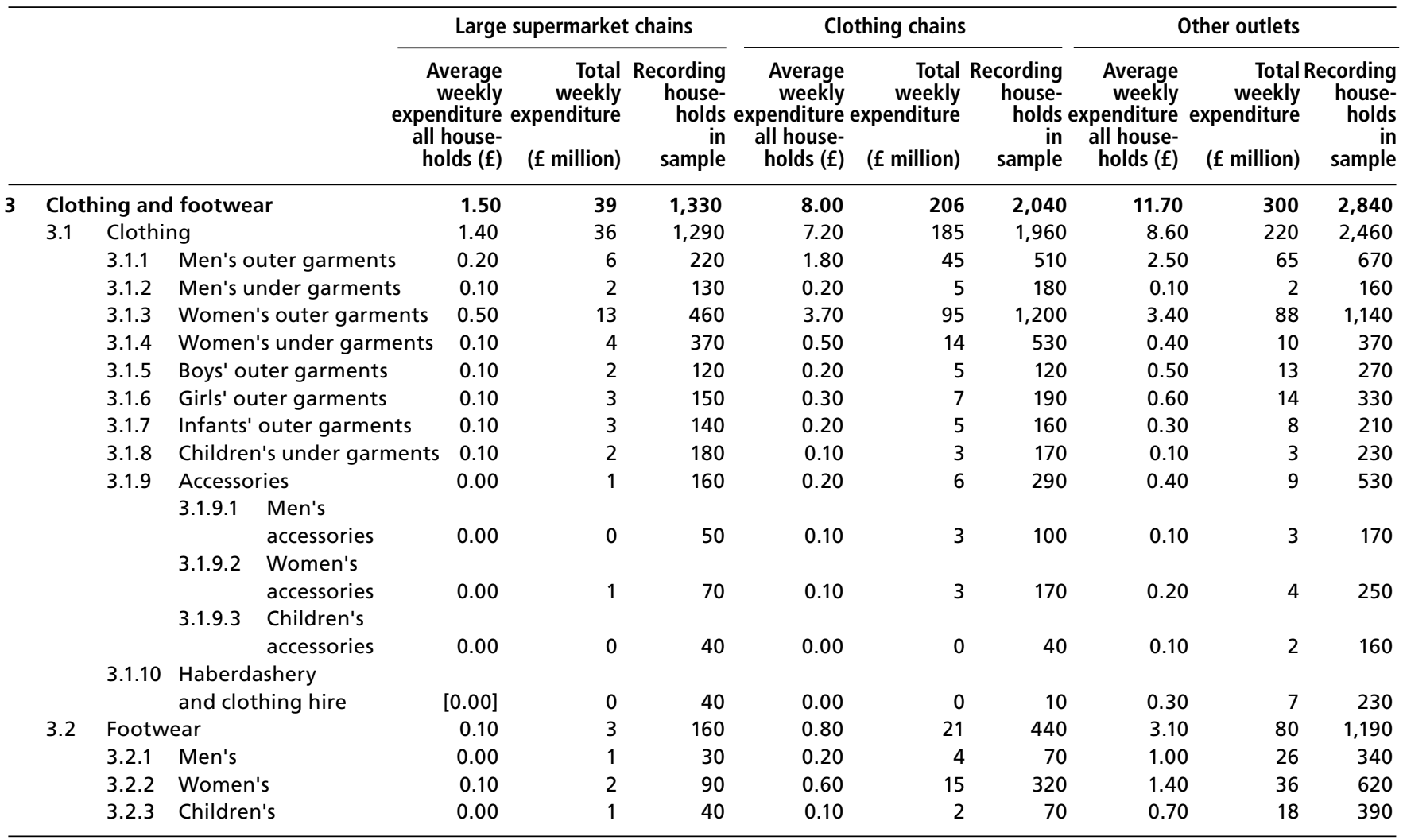

Note: The commodity and service categories are not comparable with those in publications before $2001-02$.

The numbering is sequential, it does not use actual COICOP codes.

Please see page xiii for symbols and conventions used in this report. 


\section{Table A6}

Household expenditure by gross income decile group, 2008

based on weighted data and including children's expenditure

\begin{tabular}{|c|c|c|c|c|c|c|c|}
\hline & & $\begin{array}{r}\text { Lowest } \\
\text { ten } \\
\text { per cent }\end{array}$ & $\begin{array}{l}\text { Second } \\
\text { decile } \\
\text { group }\end{array}$ & $\begin{array}{l}\text { Third } \\
\text { decile } \\
\text { group }\end{array}$ & $\begin{array}{l}\text { Fourth } \\
\text { decile } \\
\text { group }\end{array}$ & $\begin{array}{r}\text { Fifth } \\
\text { decile } \\
\text { group }\end{array}$ & $\begin{array}{l}\text { Sixth } \\
\text { decile } \\
\text { group }\end{array}$ \\
\hline \multicolumn{2}{|c|}{ Lower boundary of group ( $f$ per week) } & & 146 & 224 & 305 & 408 & 522 \\
\hline \multicolumn{2}{|c|}{ Weighted number of households (thousands) } & 2,570 & 2,570 & 2,570 & 2,570 & 2,570 & 2,570 \\
\hline \multicolumn{2}{|r|}{ Total number of households in sample } & 550 & 580 & 610 & 630 & 590 & 600 \\
\hline \multicolumn{2}{|c|}{ Total number of persons in sample } & 710 & 970 & 1,130 & 1,320 & 1,380 & 1,520 \\
\hline \multicolumn{2}{|r|}{ Weighted average number of persons per household } & 1.3 & 1.7 & 1.8 & 2.1 & 2.3 & 2.5 \\
\hline \multicolumn{2}{|c|}{ Commodity or service } & \multicolumn{6}{|c|}{ Average weekly household expenditure (f) } \\
\hline 1 & Food \& non-alcoholic drinks & 26.40 & 33.00 & 37.90 & 43.70 & 50.10 & 52.10 \\
\hline 2 & Alcoholic drinks, tobacco \& narcotics & 6.70 & 6.10 & 7.60 & 9.90 & 10.30 & 11.80 \\
\hline 3 & Clothing \& footwear & 6.40 & 8.60 & 10.60 & 14.40 & 16.60 & 21.10 \\
\hline 5 & Household goods \& services & 9.10 & 15.20 & 19.30 & 21.60 & 24.10 & 25.20 \\
\hline 6 & Health & 1.20 & 3.20 & 3.10 & 3.90 & 5.20 & 5.10 \\
\hline 7 & Transport & 12.10 & 17.10 & 24.10 & 38.90 & 51.90 & 61.00 \\
\hline 8 & Communication & 5.80 & 7.10 & 7.90 & 9.10 & 12.20 & 13.10 \\
\hline 9 & Recreation \& culture & 15.30 & 23.90 & 31.70 & 39.30 & 50.70 & 69.30 \\
\hline 10 & Education & {$[1.40]$} & {$[0.50]$} & 0.50 & 0.80 & 2.60 & 2.40 \\
\hline 11 & Restaurants \& hotels & 9.00 & 12.00 & 14.80 & 23.80 & 28.20 & 34.10 \\
\hline 12 & Miscellaneous goods \& services & 10.40 & 15.40 & 19.10 & 21.30 & 28.10 & 33.90 \\
\hline $1-12$ & All expenditure groups & 140.10 & 180.50 & 224.50 & 278.00 & 333.90 & 385.10 \\
\hline 13 & Other expenditure items & 13.70 & 19.50 & 32.60 & 52.10 & 59.10 & 74.10 \\
\hline \multicolumn{8}{|c|}{ Average weekly expenditure per person (f) } \\
\hline Tota & expenditure & 117.70 & 119.20 & 142.20 & 157.00 & 169.50 & 184.60 \\
\hline
\end{tabular}

Note: The commodity and service categories are not comparable to those in publications before 2001-02.

Please see page xiii for symbols and conventions used in this report.

1 Excluding mortgage interest payments, council tax and Northern Ireland rates. 


\section{Table A6}

Household expenditure by gross income decile group, 2008 (cont.)

based on weighted data and including children's expenditure

\begin{tabular}{|c|c|c|c|c|c|c|}
\hline & & $\begin{array}{r}\text { Seventh } \\
\text { decile } \\
\text { group }\end{array}$ & $\begin{array}{l}\text { Eighth } \\
\text { decile } \\
\text { group }\end{array}$ & $\begin{array}{l}\text { Ninth } \\
\text { decile } \\
\text { group }\end{array}$ & $\begin{array}{r}\text { Highest } \\
\text { ten } \\
\text { per cent }\end{array}$ & $\begin{array}{r}\text { All } \\
\text { house- } \\
\text { holds }\end{array}$ \\
\hline \multicolumn{2}{|c|}{ Lower boundary of group (f per week) } & 664 & 817 & 1026 & 1,356 & \\
\hline \multicolumn{2}{|c|}{ Weighted number of households (thousands) } & 2,570 & 2,560 & 2,570 & 2,560 & 25,690 \\
\hline \multicolumn{2}{|r|}{ Total number of households in sample } & 590 & 570 & 550 & 580 & 5,850 \\
\hline \multicolumn{2}{|c|}{ Total number of persons in sample } & 1,620 & 1,640 & 1,680 & 1,860 & 13,830 \\
\hline \multicolumn{2}{|r|}{ Weighted average number of persons per household } & 2.8 & 2.8 & 3.1 & 3.2 & 2.4 \\
\hline \multicolumn{2}{|c|}{ Commodity or service } & \multicolumn{5}{|c|}{ Average weekly household expenditure (f) } \\
\hline 1 & Food \& non-alcoholic drinks & 55.20 & 63.40 & 65.90 & 79.40 & 50.70 \\
\hline 2 & Alcoholic drinks, tobacco \& narcotics & 12.10 & 14.60 & 13.30 & 15.50 & 10.80 \\
\hline 3 & Clothing \& footwear & 23.70 & 29.60 & 36.00 & 48.80 & 21.60 \\
\hline 4 & Housing(net) ${ }^{1}$, fuel \& power & 57.10 & 63.00 & 54.80 & 72.00 & 53.00 \\
\hline 5 & Household goods \& services & 34.60 & 37.70 & 46.50 & 68.10 & 30.10 \\
\hline 6 & Health & 4.50 & 7.00 & 7.10 & 10.90 & 5.10 \\
\hline 7 & Transport & 73.60 & 81.90 & 112.20 & 161.10 & 63.40 \\
\hline 8 & Communication & 14.10 & 14.90 & 16.70 & 18.70 & 12.00 \\
\hline 9 & Recreation \& culture & 69.00 & 75.80 & 86.50 & 139.30 & 60.10 \\
\hline 10 & Education & 4.80 & 7.30 & 7.10 & 34.60 & 6.20 \\
\hline 11 & Restaurants \& hotels & 45.50 & 53.20 & 60.50 & 96.30 & 37.70 \\
\hline 12 & Miscellaneous goods \& services & 38.00 & 53.50 & 57.10 & 79.60 & 35.60 \\
\hline $1-12$ & All expenditure groups & 432.30 & 501.90 & 563.60 & 824.40 & 386.30 \\
\hline 13 & Other expenditure items & 104.50 & 123.80 & 146.80 & 220.50 & 84.60 \\
\hline \multicolumn{7}{|c|}{ Average weekly expenditure per person (f) } \\
\hline \multicolumn{2}{|c|}{ Total expenditure } & 192.80 & 223.10 & 232.70 & 323.20 & 199.80 \\
\hline
\end{tabular}

Note: The commodity and service categories are not comparable to those in publications before 2001-02.

Please see page xiii for symbols and conventions used in this report.

1 Excluding mortgage interest payments, council tax and Northern Ireland rates. 


\section{Table A7}

\section{Household expenditure as a percentage of total expenditure}

by gross income decile group, 2008

based on weighted data and including children's expenditure

\begin{tabular}{|c|c|c|c|c|c|c|c|}
\hline & & $\begin{array}{r}\text { Lowest } \\
\text { ten } \\
\text { per cent }\end{array}$ & $\begin{array}{l}\text { Second } \\
\text { decile } \\
\text { group }\end{array}$ & $\begin{array}{l}\text { Third } \\
\text { decile } \\
\text { group }\end{array}$ & $\begin{array}{l}\text { Fourth } \\
\text { decile } \\
\text { group }\end{array}$ & $\begin{array}{l}\text { Fifth } \\
\text { decile } \\
\text { group }\end{array}$ & $\begin{array}{r}\text { Sixth } \\
\text { decile } \\
\text { group }\end{array}$ \\
\hline \multicolumn{2}{|c|}{ Lower boundary of group ( $f$ per week) } & & 146 & 224 & 305 & 408 & 522 \\
\hline \multicolumn{2}{|c|}{ Weighted number of households (thousands) } & 2,570 & 2,570 & 2,570 & 2,570 & 2,570 & 2,570 \\
\hline \multicolumn{2}{|c|}{ Total number of households in sample } & 550 & 580 & 610 & 630 & 590 & 600 \\
\hline \multicolumn{2}{|c|}{ Total number of persons in sample } & 710 & 970 & 1,130 & 1,320 & 1,380 & 1,520 \\
\hline \multirow{2}{*}{\multicolumn{2}{|c|}{$\begin{array}{l}\text { Total number of adults in sample } \\
\text { Weighted average number of persons per household }\end{array}$}} & 600 & 770 & 920 & 1,040 & 1,070 & 1,160 \\
\hline & & 1.3 & 1.7 & 1.8 & 2.1 & 2.3 & 2.5 \\
\hline \multicolumn{2}{|c|}{ Commodity or service } & \multicolumn{6}{|c|}{ Percentage of total expenditure } \\
\hline 1 & Food \& non-alcoholic drinks & 17 & 16 & 15 & 13 & 13 & 11 \\
\hline 2 & Alcoholic drinks, tobacco \& narcotics & 4 & 3 & 3 & 3 & 3 & 3 \\
\hline 3 & Clothing \& footwear & 4 & 4 & 4 & 4 & 4 & 5 \\
\hline 4 & Housing(net) ${ }^{1}$, fuel \& power & 24 & 19 & 19 & 16 & 14 & 12 \\
\hline 5 & Household goods \& services & 6 & 8 & 8 & 7 & 6 & 5 \\
\hline 6 & Health & 1 & 2 & 1 & 1 & 1 & 1 \\
\hline 7 & Transport & 8 & 9 & 9 & 12 & 13 & 13 \\
\hline 8 & Communication & 4 & 4 & 3 & 3 & 3 & 3 \\
\hline 9 & Recreation \& culture & 10 & 12 & 12 & 12 & 13 & 15 \\
\hline 10 & Education & {$[1]$} & [0] & 0 & 0 & 1 & 1 \\
\hline 11 & Restaurants \& hotels & 6 & 6 & 6 & 7 & 7 & 7 \\
\hline 12 & Miscellaneous goods \& services & 7 & 8 & 7 & 6 & 7 & 7 \\
\hline $1-12$ & All expenditure groups & 91 & 90 & 87 & 84 & 85 & 84 \\
\hline 13 & Other expenditure items & 9 & 10 & 13 & 16 & 15 & 16 \\
\hline
\end{tabular}

Note: The commodity and service categories are not comparable to those in publications before 2001-02. Please see page xiii for symbols and conventions used in this report.

1 Excluding mortgage interest payments, council tax and Northern Ireland rates. 


\section{Table A7}

\section{Household expenditure as a percentage of total expenditure by gross income decile group, 2008 (cont.)}

based on weighted data and including children's expenditure

\begin{tabular}{|c|c|c|c|c|c|c|}
\hline & & $\begin{array}{r}\text { Seventh } \\
\text { decile } \\
\text { group }\end{array}$ & $\begin{array}{l}\text { Eighth } \\
\text { decile } \\
\text { group }\end{array}$ & $\begin{array}{l}\text { Ninth } \\
\text { decile } \\
\text { group }\end{array}$ & $\begin{array}{r}\text { Highest } \\
\text { ten } \\
\text { per cent }\end{array}$ & $\begin{array}{r}\text { All } \\
\text { house- } \\
\text { holds }\end{array}$ \\
\hline \multicolumn{2}{|c|}{ Lower boundary of group ( $f$ per week) } & 664 & 817 & 1026 & 1,356 & \\
\hline \multirow{2}{*}{\multicolumn{2}{|c|}{$\begin{array}{l}\text { Weighted number of households (thousands) } \\
\text { Total number of households in sample }\end{array}$}} & 2,570 & 2,560 & 2,570 & 2,560 & 25,690 \\
\hline & & 590 & 570 & 550 & 580 & 5,850 \\
\hline \multicolumn{2}{|c|}{ Total number of persons in sample } & 1,620 & 1,640 & 1,680 & 1,860 & 13,830 \\
\hline \multirow{2}{*}{\multicolumn{2}{|c|}{$\begin{array}{l}\text { Total number of adults in sample } \\
\text { Weighted average number of persons per household }\end{array}$}} & 1,190 & 1,240 & 1,280 & 1,380 & 10,640 \\
\hline & & 2.8 & 2.8 & 3.1 & 3.2 & 2.4 \\
\hline \multicolumn{2}{|c|}{ Commodity or service } & \multicolumn{5}{|c|}{ Percentage of total expenditure } \\
\hline 1 & Food \& non-alcoholic drinks & 10 & 10 & 9 & 8 & 11 \\
\hline 2 & Alcoholic drinks, tobacco \& narcotics & 2 & 2 & 2 & 1 & 2 \\
\hline 3 & Clothing \& footwear & 4 & 5 & 5 & 5 & 5 \\
\hline 4 & Housing(net)', fuel \& power & 11 & 10 & 8 & 7 & 11 \\
\hline 5 & Household goods \& services & 6 & 6 & 7 & 7 & 6 \\
\hline 6 & Health & 1 & 1 & 1 & 1 & 1 \\
\hline 7 & Transport & 14 & 13 & 16 & 15 & 13 \\
\hline 8 & Communication & 3 & 2 & 2 & 2 & 3 \\
\hline 9 & Recreation \& culture & 13 & 12 & 12 & 13 & 13 \\
\hline 10 & Education & 1 & 1 & 1 & 3 & 1 \\
\hline 11 & Restaurants \& hotels & 8 & 8 & 9 & 9 & 8 \\
\hline 12 & Miscellaneous goods \& services & 7 & 9 & 8 & 8 & 8 \\
\hline $1-12$ & All expenditure groups & 81 & 80 & 79 & 79 & 82 \\
\hline 13 & Other expenditure items & 19 & 20 & 21 & 21 & 18 \\
\hline Tota & expenditure & 100 & 100 & 100 & 100 & 100 \\
\hline
\end{tabular}

Note: The commodity and service categories are not comparable to those in publications before 2001-02. Please see page xiii for symbols and conventions used in this report.

1 Excluding mortgage interest payments, council tax and Northern Ireland rates. 


\section{Table A8}

Detailed household expenditure by gross income decile group, 2008

based on weighted data and including children's expenditure

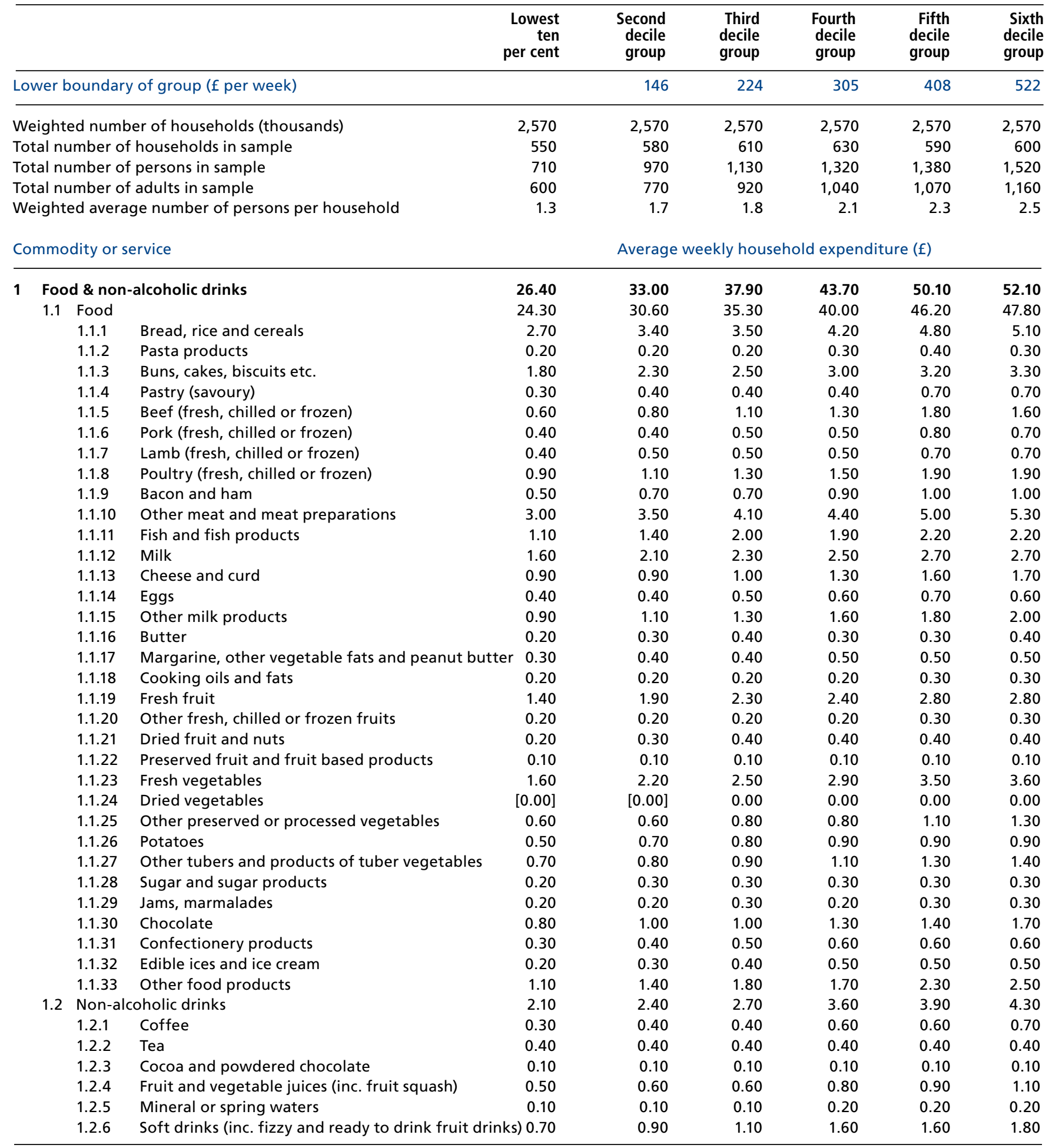

Note: The commodity and service categories are not comparable to those in publications before 2001-02.

The numbering system is sequential, it does not use actual COICOP codes.

Please see page xiii for symbols and conventions used in this report. 


\section{Table A8}

Detailed household expenditure by gross income decile group, 2008 (cont.)

based on weighted data and including children's expenditure

\begin{tabular}{|c|c|c|c|c|c|c|c|}
\hline & & & $\begin{array}{r}\text { Seventh } \\
\text { decile } \\
\text { group }\end{array}$ & $\begin{array}{l}\text { Eighth } \\
\text { decile } \\
\text { group }\end{array}$ & $\begin{array}{l}\text { Ninth } \\
\text { decile } \\
\text { group }\end{array}$ & $\begin{array}{r}\text { Highest } \\
\text { ten } \\
\text { per cent }\end{array}$ & $\begin{array}{r}\text { All } \\
\text { house- } \\
\text { holds }\end{array}$ \\
\hline Lower $\mathrm{k}$ & ounda & of group ( $\mathrm{f}$ per week) & 664 & 817 & 1026 & 1,356 & \\
\hline Weight & ed num & er of households (thousands) & 2,570 & 2,560 & 2,570 & 2,560 & 25,690 \\
\hline Total nu & umber & households in sample & 590 & 570 & 550 & 580 & 5,850 \\
\hline Total ni & umber $\mathrm{c}$ & persons in sample & 1,620 & 1,640 & 1,680 & 1,860 & 13,830 \\
\hline Total nu & Imber $\mathrm{c}$ & adults in sample & 1,190 & 1,240 & 1,280 & 1,380 & 10,640 \\
\hline Weight & ed aver & ge number of persons per household & 2.8 & 2.8 & 3.1 & 3.2 & 2.4 \\
\hline Commo & dity or & ervice & & age wee & ousehol & xpenditu & \\
\hline 1 Foo & d \& nor & alcoholic drinks & 55.20 & 63.40 & 65.90 & 79.40 & 50.70 \\
\hline 1.1 & Food & & 50.70 & 58.10 & 60.50 & 73.10 & 46.70 \\
\hline & 1.1.1 & Bread, rice and cereals & 5.40 & 6.10 & 6.20 & 7.20 & 4.90 \\
\hline & 1.1 .2 & Pasta products & 0.40 & 0.50 & 0.50 & 0.60 & 0.40 \\
\hline & 1.1 .3 & Buns, cakes, biscuits etc. & 3.60 & 3.70 & 4.00 & 4.50 & 3.20 \\
\hline & 1.1 .4 & Pastry (savoury) & 0.70 & 0.90 & 1.10 & 1.20 & 0.70 \\
\hline & 1.1 .5 & Beef (fresh, chilled or frozen) & 1.80 & 2.00 & 2.20 & 2.80 & 1.60 \\
\hline & 1.1 .6 & Pork (fresh, chilled or frozen) & 0.70 & 0.80 & 0.70 & 0.90 & 0.60 \\
\hline & 1.1.7 & Lamb (fresh, chilled or frozen) & 0.60 & 0.80 & 0.70 & 1.10 & 0.60 \\
\hline & 1.1 .8 & Poultry (fresh, chilled or frozen) & 2.00 & 2.50 & 2.60 & 3.30 & 1.90 \\
\hline & 1.1.9 & Bacon and ham & 1.00 & 1.10 & 1.10 & 1.40 & 0.90 \\
\hline & 1.1 .10 & Other meat and meat preparations & 5.60 & 6.30 & 6.80 & 7.70 & 5.20 \\
\hline & 1.1.11 & Fish and fish products & 2.20 & 3.20 & 2.80 & 4.00 & 2.30 \\
\hline & 1.1.12 & Milk & 3.00 & 3.10 & 3.10 & 3.10 & 2.60 \\
\hline & 1.1 .13 & Cheese and curd & 1.90 & 2.20 & 2.30 & 2.80 & 1.70 \\
\hline & 1.1 .14 & Eggs & 0.70 & 0.70 & 0.80 & 0.90 & 0.60 \\
\hline & 1.1.15 & Other milk products & 2.10 & 2.50 & 2.50 & 2.90 & 1.90 \\
\hline & 1.1.16 & Butter & 0.40 & 0.40 & 0.40 & 0.60 & 0.40 \\
\hline & 1.1.17 & Margarine, other vegetable fats and peanut butter & 0.50 & 0.50 & 0.50 & 0.60 & 0.50 \\
\hline & 1.1.18 & Cooking oils and fats & 0.30 & 0.30 & 0.30 & 0.50 & 0.30 \\
\hline & 1.1 .19 & Fresh fruit & 3.30 & 3.60 & 3.90 & 5.30 & 3.00 \\
\hline & 1.1 .20 & Other fresh, chilled or frozen fruits & 0.40 & 0.50 & 0.60 & 0.80 & 0.40 \\
\hline & 1.1.21 & Dried fruit and nuts & 0.50 & 0.70 & 0.70 & 0.80 & 0.50 \\
\hline & 1.1 .22 & Preserved fruit and fruit based products & 0.10 & 0.10 & 0.10 & 0.20 & 0.10 \\
\hline & 1.1 .23 & Fresh vegetables & 3.90 & 5.00 & 5.20 & 6.90 & 3.70 \\
\hline & 1.1 .24 & Dried vegetables & 0.10 & 0.10 & 0.00 & 0.10 & 0.00 \\
\hline & 1.1 .25 & Other preserved or processed vegetables & 1.20 & 1.50 & 1.70 & 2.00 & 1.20 \\
\hline & 1.1 .26 & Potatoes & 0.90 & 1.00 & 1.10 & 1.20 & 0.90 \\
\hline & 1.1.27 & Other tubers and products of tuber vegetables & 1.50 & 1.50 & 1.60 & 1.70 & 1.30 \\
\hline & 1.1 .28 & Sugar and sugar products & 0.30 & 0.40 & 0.30 & 0.40 & 0.30 \\
\hline & 1.1 .29 & Jams, marmalades & 0.20 & 0.30 & 0.30 & 0.40 & 0.30 \\
\hline & 1.1 .30 & Chocolate & 1.70 & 1.70 & 2.00 & 2.10 & 1.50 \\
\hline & 1.1.31 & Confectionery products & 0.60 & 0.70 & 0.60 & 0.70 & 0.60 \\
\hline & 1.1.32 & Edible ices and ice cream & 0.60 & 0.50 & 0.70 & 0.70 & 0.50 \\
\hline & 1.1.33 & Other food products & 2.30 & 2.80 & 2.80 & 3.80 & 2.30 \\
\hline 1.2 & Non-a & oholic drinks & 4.50 & 5.20 & 5.40 & 6.30 & 4.00 \\
\hline & 1.2.1 & Coffee & 0.60 & 0.60 & 0.70 & 0.80 & 0.60 \\
\hline & 1.2 .2 & Tea & 0.40 & 0.50 & 0.50 & 0.50 & 0.40 \\
\hline & 1.2 .3 & Cocoa and powdered chocolate & 0.10 & 0.10 & 0.10 & 0.10 & 0.10 \\
\hline & 1.2 .4 & Fruit and vegetable juices, mineral waters & 1.30 & 1.50 & 1.80 & 2.20 & 1.10 \\
\hline & 1.2 .5 & Mineral or spring waters & 0.20 & 0.30 & 0.30 & 0.50 & 0.20 \\
\hline & 1.2 .6 & Soft drinks (inc. fizzy and ready to drink fruit drinks) & 1.80 & 2.10 & 2.10 & 2.20 & 1.60 \\
\hline
\end{tabular}

Note: The commodity and service categories are not comparable to those in publications before 2001-02.

The numbering system is sequential, it does not use actual COICOP codes.

Please see page xiii for symbols and conventions used in this report. 


\section{Table A8}

Detailed household expenditure by gross income decile group, 2008 (cont.)

based on weighted data and including children's expenditure

\begin{tabular}{|c|c|c|c|c|c|c|c|c|c|}
\hline & & & & $\begin{array}{r}\text { Lowest } \\
\text { ten } \\
\text { per cent }\end{array}$ & $\begin{array}{l}\text { Second } \\
\text { decile } \\
\text { group }\end{array}$ & $\begin{array}{l}\text { Third } \\
\text { decile } \\
\text { group }\end{array}$ & $\begin{array}{l}\text { Fourth } \\
\text { decile } \\
\text { group }\end{array}$ & $\begin{array}{l}\text { Fifth } \\
\text { decile } \\
\text { group }\end{array}$ & $\begin{array}{l}\text { Sixth } \\
\text { decile } \\
\text { group }\end{array}$ \\
\hline \multicolumn{5}{|c|}{ Commodity or service } & \multicolumn{5}{|c|}{ Average weekly household expenditure $(f)$} \\
\hline \multirow[t]{9}{*}{2} & \multicolumn{3}{|c|}{ Alcoholic drink, tobacco \& narcotics } & 6.70 & 6.10 & 7.60 & 9.90 & 10.30 & 11.80 \\
\hline & \multicolumn{3}{|c|}{2.1 Alcoholic drinks } & 2.40 & 3.10 & 3.60 & 4.90 & 5.20 & 6.00 \\
\hline & & 2.1 .1 & Spirits and liqueurs (brought home) & 0.50 & 0.90 & 1.00 & 1.20 & 1.40 & 1.30 \\
\hline & & 2.1 .2 & Wines, fortified wines (brought home) & 0.90 & 1.10 & 1.60 & 2.40 & 2.20 & 2.70 \\
\hline & & 2.1 .3 & Beer, lager, ciders and perry (brought home) & 1.00 & 1.00 & 1.00 & 1.20 & 1.50 & 1.90 \\
\hline & & 2.1 .4 & Alcopops (brought home) & {$[0.00]$} & {$[0.00]$} & {$[0.00]$} & {$[0.10]$} & {$[0.10]$} & [0.10] \\
\hline & \multirow[t]{3}{*}{2.2} & Tobac & co and narcotics & 4.30 & 3.10 & 4.00 & 4.90 & 5.10 & 5.80 \\
\hline & & 2.2 .1 & Cigarettes & 3.40 & 2.20 & 3.40 & 4.30 & 4.30 & 4.80 \\
\hline & & 2.2 .2 & Cigars, other tobacco products and narcotics & 0.90 & 0.80 & 0.60 & 0.60 & 0.80 & 1.00 \\
\hline \multirow[t]{14}{*}{3} & \multicolumn{3}{|c|}{ Clothing \& footwear } & 6.40 & 8.60 & 10.60 & 14.40 & 16.60 & 21.10 \\
\hline & \multirow[t]{12}{*}{3.1} & \multicolumn{2}{|c|}{ Clothing } & 5.10 & 6.90 & 8.70 & 11.30 & 13.00 & 16.70 \\
\hline & & 3.1.1 & Men's outer garments & 1.30 & 1.10 & 1.60 & 1.90 & 2.50 & 4.10 \\
\hline & & 3.1 .2 & Men's under garments & 0.10 & 0.20 & 0.10 & 0.30 & 0.20 & 0.30 \\
\hline & & 3.1 .3 & Women's outer garments & 2.40 & 3.20 & 4.40 & 4.60 & 6.00 & 7.30 \\
\hline & & 3.1.4 & Women's under garments & 0.40 & 0.50 & 0.50 & 0.80 & 0.90 & 1.30 \\
\hline & & 3.1.5 & Boys' outer garments (5-15) & [0.20] & 0.20 & 0.60 & 0.80 & 0.60 & 0.60 \\
\hline & & 3.1 .6 & Girls' outer garments (5-15) & {$[0.10]$} & 0.50 & 0.30 & 1.10 & 1.20 & 0.90 \\
\hline & & 3.1.7 & Infants' outer garments (under 5) & 0.20 & 0.40 & 0.40 & 0.50 & 0.50 & 0.60 \\
\hline & & 3.1 .8 & Children's under garments (under 16) & [0.10] & 0.10 & 0.20 & 0.30 & 0.30 & 0.30 \\
\hline & & 3.1.9 & Accessories & 0.10 & 0.40 & 0.20 & 0.70 & 0.60 & 0.50 \\
\hline & & 3.1 .10 & Haberdashery and clothing hire & {$[0.10]$} & 0.10 & 0.10 & 0.10 & 0.20 & 0.70 \\
\hline & & 3.1.11 & Dry cleaners, laundry and dyeing & [0.10] & {$[0.00]$} & {$[0.20]$} & {$[0.10]$} & [0.10] & [0.10] \\
\hline & 3.2 & Footh & ear & 1.30 & 1.70 & 1.90 & 3.10 & 3.70 & 4.40 \\
\hline \multirow[t]{13}{*}{4} & \multicolumn{3}{|c|}{ Housing (net)', fuel \& power } & 36.30 & 38.40 & 47.90 & 51.30 & 53.80 & 55.90 \\
\hline & \multirow[t]{5}{*}{4.1} & \multicolumn{2}{|c|}{ Actual rentals for housing } & 63.40 & 47.90 & 42.00 & 32.50 & 27.40 & 24.10 \\
\hline & & 4.1.1 & Gross rent & 63.40 & 47.90 & 42.00 & 32.50 & 27.40 & 24.10 \\
\hline & & 4.1.2 & less housing benefit, rebates \& allowances rec'd & 48.00 & 33.20 & 21.20 & 10.90 & 4.60 & 1.10 \\
\hline & & 4.1 .3 & Net rent ${ }^{2}$ & 15.40 & 14.70 & 20.90 & 21.60 & 22.80 & 23.00 \\
\hline & & 4.1.4 & Second dwelling rent & - & - & - & - & - & - \\
\hline & 4.2 & Maint & enance and repair of dwelling & 2.10 & 2.50 & 3.90 & 5.20 & 6.00 & 6.60 \\
\hline & \multirow[t]{2}{*}{4.3} & \multicolumn{2}{|c|}{ Water supply and miscellaneous services } & & & & & & \\
\hline & & relatir & g to the dwelling & 5.80 & 6.40 & 7.50 & 7.30 & 7.40 & 7.50 \\
\hline & \multirow[t]{4}{*}{4.4} & \multicolumn{2}{|c|}{ Electricity, gas and other fuels } & 12.90 & 14.70 & 15.60 & 17.20 & 17.60 & 18.80 \\
\hline & & 4.4.1 & Electricity & 6.30 & 7.10 & 7.80 & 8.20 & 8.60 & 9.20 \\
\hline & & 4.4 .2 & Gas & 5.50 & 6.30 & 6.60 & 7.40 & 7.60 & 8.00 \\
\hline & & 4.4 .3 & Other fuels & 1.10 & 1.30 & 1.20 & 1.60 & 1.40 & 1.60 \\
\hline
\end{tabular}

Note: The commodity and service categories are not comparable to those in publications before 2001-02.

The numbering system is sequential, it does not use actual COICOP codes.

Please see page xiii for symbols and conventions used in this report.

1 Excluding mortgage interest payments, council tax and Northern Ireland rates.

2 The figure included in total expenditure is net rent as opposed to gross rent. 


\section{Table A8}

Detailed household expenditure by gross income decile group, 2008 (cont.)

based on weighted data and including children's expenditure

\begin{tabular}{|c|c|c|c|c|c|c|c|c|}
\hline & & & & $\begin{array}{r}\text { Seventh } \\
\text { decile } \\
\text { group }\end{array}$ & $\begin{array}{l}\text { Eighth } \\
\text { decile } \\
\text { group }\end{array}$ & $\begin{array}{l}\text { Ninth } \\
\text { decile } \\
\text { group }\end{array}$ & $\begin{array}{r}\text { Highest } \\
\text { ten } \\
\text { per cent }\end{array}$ & $\begin{array}{r}\text { All } \\
\text { house- } \\
\text { holds }\end{array}$ \\
\hline \multicolumn{4}{|c|}{ Commodity or service } & \multicolumn{5}{|c|}{ Average weekly household expenditure (f) } \\
\hline \multirow[t]{9}{*}{2} & \multicolumn{3}{|c|}{ Alcoholic drink, tobacco \& narcotics } & 12.10 & 14.60 & 13.30 & 15.50 & 10.80 \\
\hline & \multicolumn{3}{|c|}{ 2.1 Alcoholic drinks } & 7.20 & 9.50 & 8.90 & 11.40 & 6.20 \\
\hline & & 2.1 .1 & Spirits and liqueurs (brought home) & 1.40 & 1.50 & 1.50 & 1.20 & 1.20 \\
\hline & & 2.1 .2 & Wines, fortified wines (brought home) & 3.40 & 5.60 & 4.80 & 7.80 & 3.30 \\
\hline & & 2.1 .3 & Beer, lager, ciders and perry (brought home) & 2.40 & 2.40 & 2.50 & 2.30 & 1.70 \\
\hline & & 2.1 .4 & Alcopops (brought home) & [0.10] & [0.10] & {$[0.10]$} & {$[0.10]$} & 0.10 \\
\hline & \multirow[t]{3}{*}{2.2} & Tobac & $\mathrm{o}$ and narcotics & 4.90 & 5.10 & 4.50 & 4.00 & 4.60 \\
\hline & & 2.2 .1 & Cigarettes & 4.20 & 4.50 & 4.10 & 3.70 & 3.90 \\
\hline & & 2.2 .2 & Cigars, other tobacco products and narcotics & 0.70 & 0.60 & 0.40 & [0.30] & 0.70 \\
\hline \multirow[t]{14}{*}{3} & \multicolumn{3}{|c|}{ Clothing \& footwear } & 23.70 & 29.60 & 36.00 & 48.80 & 21.60 \\
\hline & \multicolumn{3}{|c|}{ 3.1 Clothing } & 19.70 & 23.90 & 29.70 & 39.70 & 17.50 \\
\hline & & 3.1 .1 & Men's outer garments & 5.00 & 6.20 & 10.40 & 11.30 & 4.50 \\
\hline & & 3.1 .2 & Men's under garments & 0.40 & 0.50 & 0.70 & 0.60 & 0.30 \\
\hline & & 3.1 .3 & Women's outer garments & 7.70 & 10.60 & 12.00 & 18.20 & 7.60 \\
\hline & & 3.1 .4 & Women's under garments & 1.00 & 1.30 & 1.40 & 2.50 & 1.10 \\
\hline & & 3.1 .5 & Boys' outer garments (5-15) & 1.20 & 1.00 & 1.10 & 1.40 & 0.80 \\
\hline & & 3.1 .6 & Girls' outer garments (5-15) & 1.60 & 0.90 & 1.20 & 1.70 & 1.00 \\
\hline & & 3.1.7 & Infants' outer garments (under 5) & 1.00 & 1.00 & 0.60 & 1.00 & 0.60 \\
\hline & & 3.1 .8 & Children's under garments (under 16) & 0.40 & 0.40 & 0.40 & 0.40 & 0.30 \\
\hline & & 3.1 .9 & Accessories & 0.90 & 1.00 & 1.10 & 1.50 & 0.70 \\
\hline & & 3.1 .10 & Haberdashery and clothing hire & 0.30 & 0.60 & 0.20 & 0.30 & 0.30 \\
\hline & & 3.1.11 & Dry cleaners, laundry and dyeing & {$[0.20]$} & 0.40 & 0.50 & 1.00 & 0.30 \\
\hline & 3.2 & Footw & & 4.00 & 5.80 & 6.20 & 9.10 & 4.10 \\
\hline \multirow[t]{13}{*}{4} & \multicolumn{3}{|c|}{ Housing (net)' ${ }^{1}$ fuel \& power } & 57.10 & 63.00 & 54.80 & 72.00 & 53.00 \\
\hline & \multirow[t]{5}{*}{4.1} & \multicolumn{2}{|c|}{ Actual rentals for housing } & 22.70 & 24.30 & 12.70 & 19.00 & 31.60 \\
\hline & & 4.1.1 & Gross rent & 22.70 & 24.20 & 12.70 & 18.30 & 31.50 \\
\hline & & 4.1 .2 & less housing benefit, rebates \& allowances rec'd & 0.90 & 1.10 & 0.10 & 0.30 & 12.10 \\
\hline & & 4.1 .3 & Net rent ${ }^{2}$ & 21.80 & 23.10 & 12.60 & 18.00 & 19.40 \\
\hline & & 4.1.4 & Second dwelling rent & {$[0.00]$} & [0.10] & - & [0.70] & [0.10] \\
\hline & 4.2 & Maint & nance and repair of dwelling & 7.90 & 11.20 & 11.00 & 15.60 & 7.20 \\
\hline & \multirow[t]{2}{*}{4.3} & Water & supply and miscellaneous services & & & & & \\
\hline & & relatir & $g$ to the dwelling & 7.40 & 7.70 & 8.30 & 8.90 & 7.40 \\
\hline & \multirow[t]{4}{*}{4.4} & Electri & city, gas and other fuels & 19.90 & 21.00 & 23.00 & 28.70 & 18.90 \\
\hline & & 4.4 .1 & Electricity & 9.50 & 9.90 & 11.10 & 12.60 & 9.00 \\
\hline & & 4.4 .2 & Gas & 8.90 & 9.00 & 10.00 & 12.60 & 8.20 \\
\hline & & 4.4 .3 & Other fuels & 1.50 & 2.20 & 1.90 & 3.50 & 1.70 \\
\hline
\end{tabular}

Note: The commodity and service categories are not comparable to those in publications before $2001-02$.

The numbering system is sequential, it does not use actual COICOP codes.

Please see page xiii for symbols and conventions used in this report.

1 Excluding mortgage interest payments, council tax and Northern Ireland rates.

2 The figure included in total expenditure is net rent as opposed to gross rent. 


\section{Table A8}

Detailed household expenditure by gross income decile group, 2008 (cont.)

based on weighted data and including children's expenditure

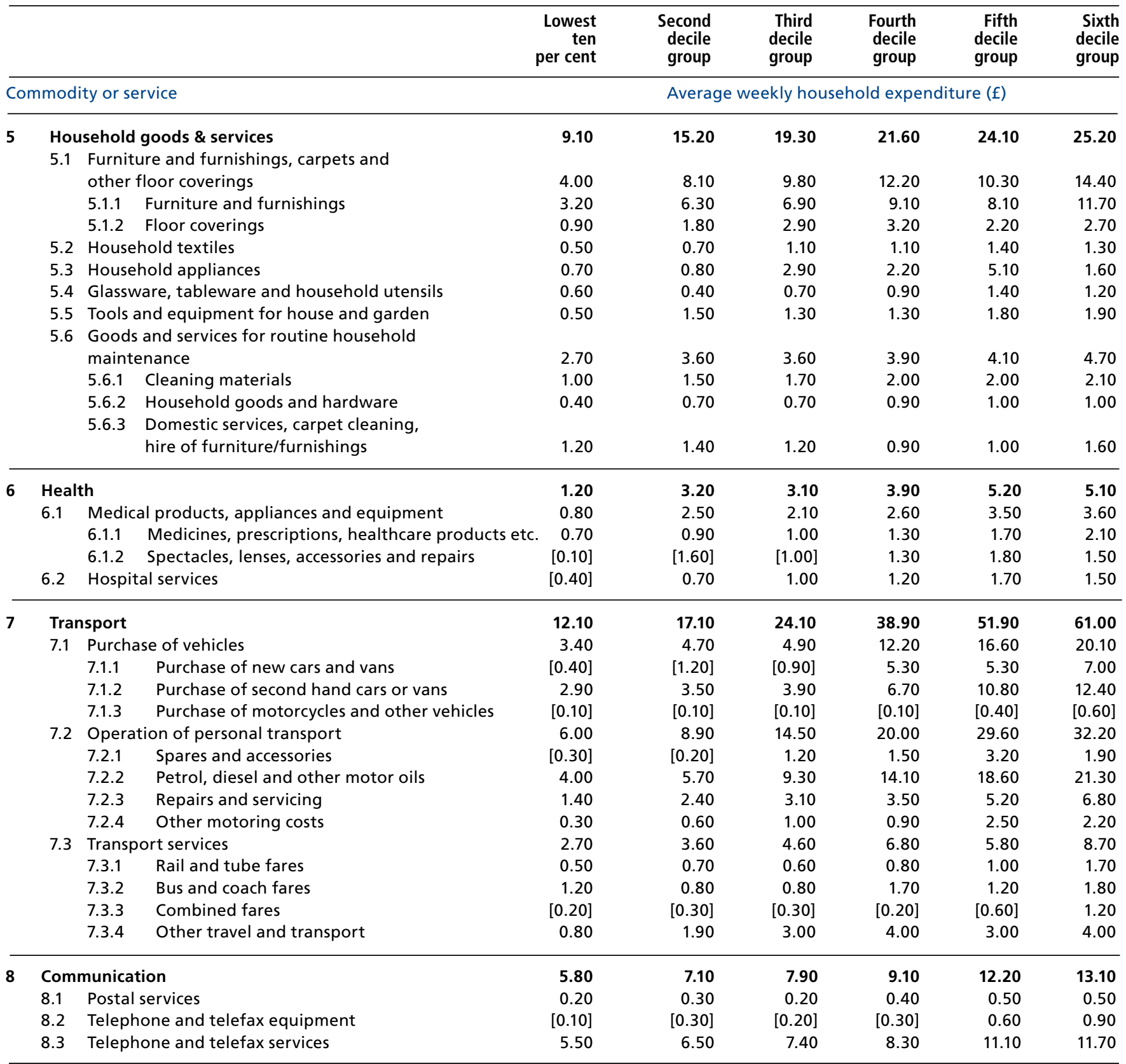

Note: The commodity and service categories are not comparable to those in publications before $2001-02$.

The numbering system is sequential, it does not use actual COICOP codes.

Please see page xiii for symbols and conventions used in this report. 


\section{Table A8}

\section{Detailed household expenditure by gross income decile group, 2008 (cont.)}

based on weighted data and including children's expenditure

\begin{tabular}{|c|c|c|c|c|c|c|c|c|}
\hline & & & & $\begin{array}{r}\text { Seventh } \\
\text { decile } \\
\text { group }\end{array}$ & $\begin{array}{l}\text { Eighth } \\
\text { decile } \\
\text { group }\end{array}$ & $\begin{array}{l}\text { Ninth } \\
\text { decile } \\
\text { group }\end{array}$ & $\begin{array}{r}\text { Highest } \\
\text { ten } \\
\text { per cent }\end{array}$ & $\begin{array}{r}\text { All } \\
\text { house- } \\
\text { holds }\end{array}$ \\
\hline & nmodit & ity or se & rvice & & age wee & ouseholc & xpenditure & \\
\hline 5 & Hous & ssehold & goods \& services & 34.60 & 37.70 & 46.50 & 68.10 & 30.10 \\
\hline & 5.1 & Furnit & re and furnishings, carpets and & & & & & \\
\hline & & other & loor coverings & 19.10 & 23.10 & 26.60 & 38.60 & 16.60 \\
\hline & & 5.1 .1 & Furniture and furnishings & 14.80 & 18.50 & 20.10 & 31.00 & 13.00 \\
\hline & & 5.1 .2 & Floor coverings & 4.30 & 4.60 & 6.50 & 7.60 & 3.70 \\
\hline & 5.2 & House & nold textiles & 2.10 & 1.50 & 2.50 & 3.20 & 1.50 \\
\hline & 5.3 & House & iold appliances & 4.00 & 3.50 & 6.40 & 7.70 & 3.50 \\
\hline & 5.4 & Glassv & are, tableware and household utensils & 1.50 & 1.90 & 2.00 & 2.50 & 1.30 \\
\hline & 5.5 & Tools & nd equipment for house and garden & 2.90 & 2.70 & 3.00 & 4.20 & 2.10 \\
\hline & 5.6 & Goods & and services for routine household & & & & & \\
\hline & & maint & nance & 5.20 & 5.00 & 6.00 & 11.80 & 5.10 \\
\hline & & 5.6 .1 & Cleaning materials & 2.40 & 2.50 & 2.80 & 3.40 & 2.20 \\
\hline & & 5.6 .2 & Household goods and hardware & 1.20 & 1.40 & 1.50 & 1.90 & 1.10 \\
\hline & & 5.6 .3 & Domestic services, carpet cleaning, & & & & & \\
\hline & & & hire/repair of furniture/furnishings & 1.60 & 1.20 & 1.70 & 6.50 & 1.80 \\
\hline 6 & Healtr & & & 4.50 & 7.00 & 7.10 & 10.90 & 5.10 \\
\hline & 6.1 & Medic & I products, appliances and equipment & 2.60 & 4.10 & 4.10 & 5.10 & 3.10 \\
\hline & & 6.1 .1 & Medicines, prescriptions, healthcare products etc. & 1.70 & 2.10 & 2.10 & 3.00 & 1.70 \\
\hline & & 6.1 .2 & Spectacles, lenses, accessories and repairs & 0.90 & 2.00 & 2.00 & 2.10 & 1.40 \\
\hline & 6.2 & Hospi & al services & 2.00 & 3.00 & 3.00 & 5.80 & 2.00 \\
\hline 7 & Trans & isport & & 73.60 & 81.90 & 112.20 & 161.10 & 63.40 \\
\hline & 7.1 & Purch & se of vehicles & 26.30 & 24.70 & 38.00 & 60.60 & 21.10 \\
\hline & & 7.1.1 & Purchase of new cars and vans & 8.80 & 6.80 & 8.30 & 22.50 & 6.60 \\
\hline & & 7.1.2 & Purchase of second hand cars or vans & 16.70 & 16.80 & 28.30 & 34.20 & 13.60 \\
\hline & & 7.1.3 & Purchase of motorcycles and other vehicles & {$[0.80]$} & [1.10] & [1.50] & [3.90] & 0.90 \\
\hline & 7.2 & Opera & ion of personal transport & 37.10 & 43.60 & 55.90 & 70.00 & 31.80 \\
\hline & & 7.2 .1 & Spares and accessories & 2.10 & 2.40 & 5.40 & 5.40 & 2.40 \\
\hline & & 7.2 .2 & Petrol, diesel and other motor oils & 25.80 & 30.10 & 36.50 & 44.90 & 21.00 \\
\hline & & 7.2 .3 & Repairs and servicing & 6.90 & 8.20 & 10.30 & 14.70 & 6.20 \\
\hline & & 7.2 .4 & Other motoring costs & 2.40 & 2.90 & 3.70 & 5.00 & 2.10 \\
\hline & 7.3 & Transp & ort services & 10.20 & 13.60 & 18.30 & 30.50 & 10.50 \\
\hline & & 7.3.1 & Rail and tube fares & 2.10 & 4.10 & 3.90 & 9.00 & 2.40 \\
\hline & & 7.3.2 & Bus and coach fares & 1.60 & 1.70 & 1.30 & 1.60 & 1.40 \\
\hline & & 7.3.3 & Combined fares & 1.90 & 1.50 & 2.30 & 3.80 & 1.20 \\
\hline & & 7.3.4 & Other travel and transport & 4.70 & 6.30 & 10.70 & 16.20 & 5.40 \\
\hline 8 & Comm & munica & ion & 14.10 & 14.90 & 16.70 & 18.70 & 12.00 \\
\hline & 8.1 & Postal & ervices & 0.70 & 0.50 & 0.60 & 0.70 & 0.50 \\
\hline & 8.2 & Teleph & one and telefax equipment & 0.60 & {$[0.60]$} & {$[0.60]$} & {$[0.60]$} & 0.50 \\
\hline & 8.3 & Teleph & one and telefax services & 12.80 & 13.80 & 15.40 & 17.40 & 11.00 \\
\hline
\end{tabular}

Note: The commodity and service categories are not comparable to those in publications before 2001-02.

The numbering system is sequential, it does not use actual COICOP codes.

Please see page xiii for symbols and conventions used in this report. 


\section{Table A8}

Detailed household expenditure by gross income decile group, 2008 (cont.)

based on weighted data and including children's expenditure

\begin{tabular}{|c|c|c|c|c|c|c|c|c|c|}
\hline & & & & $\begin{array}{r}\text { Lowest } \\
\text { ten } \\
\text { per cent }\end{array}$ & $\begin{array}{r}\text { Second } \\
\text { decile } \\
\text { group }\end{array}$ & $\begin{array}{l}\text { Third } \\
\text { decile } \\
\text { group }\end{array}$ & $\begin{array}{l}\text { Fourth } \\
\text { decile } \\
\text { group }\end{array}$ & $\begin{array}{l}\text { Fifth } \\
\text { decile } \\
\text { group }\end{array}$ & $\begin{array}{r}\text { Sixth } \\
\text { decile } \\
\text { group }\end{array}$ \\
\hline \multicolumn{5}{|c|}{ Commodity or service } & \multicolumn{5}{|c|}{ Average weekly household expenditure (f) } \\
\hline \multirow[t]{33}{*}{9} & \multicolumn{3}{|c|}{ Recreation \& culture } & 15.30 & 23.90 & 31.70 & 39.30 & 50.70 & 69.30 \\
\hline & \multirow{5}{*}{9.1} & \multicolumn{8}{|c|}{ Audio-visual, photographic and information } \\
\hline & & \multicolumn{2}{|c|}{ processing equipment } & 2.20 & 2.20 & 5.10 & 3.70 & 6.40 & 5.20 \\
\hline & & 9.1 .1 & Audio equipment and accessories, CD players & 0.50 & 0.30 & 0.70 & 0.80 & 0.80 & 1.00 \\
\hline & & 9.1 .2 & TV, video and computers & 1.50 & 1.70 & 4.20 & 2.30 & 5.10 & 3.90 \\
\hline & & 9.1 .3 & Photographic, cine and optical equipment & {$[0.20]$} & {$[0.20]$} & {$[0.10]$} & {$[0.60]$} & {$[0.40]$} & {$[0.20]$} \\
\hline & 9.2 & \multicolumn{2}{|c|}{ Other major durables for recreation and culture } & {$[0.10]$} & {$[0.30]$} & {$[1.80]$} & {$[0.80]$} & {$[0.40]$} & [15.30] \\
\hline & 9.3 & \multicolumn{8}{|c|}{ Other recreational items and equipment, } \\
\hline & & \multicolumn{2}{|c|}{ gardens and pets } & 3.00 & 5.00 & 5.30 & 8.60 & 8.30 & 12.70 \\
\hline & & 9.3 .1 & Games, toys and hobbies & 0.50 & 0.90 & 1.10 & 1.70 & 1.60 & 2.10 \\
\hline & & 9.3 .2 & Computer software and games & {$[0.40]$} & {$[0.60]$} & {$[0.60]$} & 1.30 & 1.60 & 3.20 \\
\hline & & \multirow[t]{2}{*}{9.3 .3} & Equipment for sport, camping and & & & & & & \\
\hline & & & open-air recreation & {$[0.10]$} & {$[0.20]$} & 0.20 & 0.30 & 0.40 & 1.20 \\
\hline & & 9.3 .4 & Horticultural goods, garden equipment and plants & s 0.80 & 1.40 & 1.50 & 2.50 & 1.80 & 2.60 \\
\hline & & 9.3 .5 & Pets and pet food & 1.20 & 1.90 & 1.80 & 2.70 & 2.80 & 3.50 \\
\hline & \multirow[t]{10}{*}{9.4} & \multicolumn{2}{|c|}{ Recreational and cultural services } & 6.10 & 7.90 & 8.40 & 12.20 & 16.50 & 16.90 \\
\hline & & \multirow[t]{2}{*}{9.4 .1} & Sports admissions, subscriptions, leisure class fees & & & & & & \\
\hline & & & and equipment hire & 1.00 & 1.40 & 0.90 & 2.00 & 3.00 & 4.40 \\
\hline & & 9.4 .2 & Cinema, theatre and museums etc. & 0.30 & 0.60 & 0.70 & 1.00 & 1.60 & 1.60 \\
\hline & & \multirow[t]{2}{*}{9.4 .3} & TV, video, satellite rental, cable subscriptions, & & & & & & \\
\hline & & & TV licences and the Internet & 3.00 & 3.40 & 3.70 & 4.90 & 5.80 & 6.50 \\
\hline & & 9.4 .4 & Miscellaneous entertainments & 0.20 & 0.50 & 0.40 & 0.80 & 0.90 & 1.00 \\
\hline & & \multirow[t]{2}{*}{9.4 .5} & Development of film, deposit for film development & & & & & & \\
\hline & & & passport photos, holiday and school photos & {$[0.10]$} & {$[0.10]$} & 0.10 & {$[0.10]$} & 0.30 & 0.30 \\
\hline & & 9.4 .6 & Gambling payments & 1.50 & 2.00 & 2.70 & 3.30 & 5.00 & 3.20 \\
\hline & \multirow[t]{5}{*}{9.5} & \multicolumn{2}{|c|}{ Newspapers, books and stationery } & 2.60 & 3.80 & 4.40 & 5.00 & 5.50 & 6.30 \\
\hline & & 9.5 .1 & Books & 0.30 & 0.60 & 0.80 & 0.80 & 0.90 & 1.40 \\
\hline & & 9.5 .2 & Diaries, address books, cards etc. & 0.70 & 1.10 & 1.00 & 1.40 & 1.50 & 2.10 \\
\hline & & 9.5 .3 & Newspapers & 1.10 & 1.50 & 1.90 & 2.10 & 2.20 & 1.90 \\
\hline & & 9.5 .4 & Magazines and periodicals & 0.40 & 0.60 & 0.60 & 0.70 & 0.90 & 0.90 \\
\hline & \multirow[t]{3}{*}{9.6} & \multicolumn{2}{|c|}{ Package holidays } & 1.20 & 4.70 & 6.70 & 9.10 & 13.60 & 12.90 \\
\hline & & 9.6 .1 & Package holidays - UK & {$[0.30]$} & 1.20 & 2.00 & 0.70 & 1.60 & {$[1.30]$} \\
\hline & & 9.6 .2 & Package holidays - abroad & {$[0.90]$} & 3.50 & 4.70 & 8.50 & 12.00 & 11.60 \\
\hline 10 & Educ & cation & & [1.40] & {$[0.50]$} & 0.50 & 0.80 & 2.60 & 2.40 \\
\hline & 10.1 & Educa & tion fees & {$[1.40]$} & {$[0.40]$} & {$[0.50]$} & {$[0.60]$} & 2.50 & 2.10 \\
\hline & 10.2 & $\begin{array}{l}\text { Payme } \\
\text { expen }\end{array}$ & $\begin{array}{l}\text { nts for school trips, other ad-hoc } \\
\text { diture }\end{array}$ & {$[0.00]$} & {$[0.00]$} & {$[0.10]$} & {$[0.20]$} & {$[0.10]$} & {$[0.30]$} \\
\hline 11 & Rest & taurants & \& hotels & 9.00 & 12.00 & 14.80 & 23.80 & 28.20 & 34.10 \\
\hline & 11.1 & Cateri & ng services & 8.30 & 9.90 & 12.60 & 20.80 & 24.80 & 29.40 \\
\hline & & 11.1.1 & Restaurant and café meals & 3.60 & 4.50 & 6.10 & 9.60 & 10.30 & 11.90 \\
\hline & & 11.1.2 & Alcoholic drinks (away from home) & 1.70 & 1.70 & 2.90 & 4.30 & 6.30 & 6.90 \\
\hline & & 11.1.3 & Take away meals eaten at home & 1.60 & 1.90 & 1.80 & 2.90 & 3.50 & 4.40 \\
\hline & & 11.1.4 & Other take-away and snack food & 1.00 & 1.50 & 1.40 & 2.80 & 3.40 & 4.20 \\
\hline & & 11.1.5 & Contract catering (food) and canteens & 0.40 & 0.30 & 0.50 & 1.10 & 1.40 & 2.10 \\
\hline & 11.2 & Accom & modation services & 0.70 & 2.00 & 2.20 & 3.00 & 3.40 & 4.70 \\
\hline & & 11.2.1 & Holiday in the UK & {$[0.60]$} & 0.70 & 1.00 & 1.50 & 2.30 & 2.50 \\
\hline & & 11.2.2 & Holiday abroad & {$[0.20]$} & [1.30] & [1.30] & 1.40 & 1.10 & 2.10 \\
\hline & & 11.2 .3 & Room hire & - & - & - & {$[0.10]$} & {$[0.00]$} & {$[0.00]$} \\
\hline
\end{tabular}

Note: The commodity and service categories are not comparable to those in publications before $2001-02$.

The numbering system is sequential, it does not use actual COICOP codes.

Please see page xiii for symbols and conventions used in this report. 


\section{Table A8}

\section{Detailed household expenditure by gross income decile group, 2008 (cont.)}

based on weighted data and including children's expenditure

\begin{tabular}{|c|c|c|c|c|c|c|c|c|}
\hline & $\begin{array}{r}\text { Seventh } \\
\text { decile } \\
\text { group }\end{array}$ & $\begin{array}{l}\text { Eighth } \\
\text { decile } \\
\text { group }\end{array}$ & $\begin{array}{l}\text { Ninth } \\
\text { decile } \\
\text { group }\end{array}$ & $\begin{array}{r}\text { Highest } \\
\text { ten } \\
\text { per cent }\end{array}$ & $\begin{array}{r}\text { All } \\
\text { house- } \\
\text { holds }\end{array}$ \\
\hline & \multicolumn{5}{|c|}{ Average weekly household expenditure ( $f$ ) } \\
\hline \multirow[t]{33}{*}{9} & \multicolumn{3}{|c|}{ Recreation \& culture } & 69.00 & 75.80 & 86.50 & 139.30 & 60.10 \\
\hline & \multirow[t]{5}{*}{9.1} & \multicolumn{2}{|c|}{ Audio-visual, photographic and information } & & & & & \\
\hline & & \multicolumn{2}{|c|}{ processing equipment } & 8.80 & 10.70 & 12.70 & 14.80 & 7.20 \\
\hline & & 9.1 .1 & Audio equipment and accessories, CD players & 2.00 & 1.40 & 2.00 & 3.20 & 1.30 \\
\hline & & 9.1 .2 & TV, video and computers & 5.70 & 7.00 & 7.10 & 9.50 & 4.80 \\
\hline & & 9.1 .3 & Photographic, cine and optical equipment & [1.10] & [2.20] & [3.50] & 2.00 & 1.10 \\
\hline & 9.2 & Other $\mathrm{r}$ & najor durables for recreation and culture & {$[5.00]$} & {$[1.50]$} & 1.80 & 14.40 & 4.10 \\
\hline & \multirow[t]{8}{*}{9.3} & \multicolumn{2}{|c|}{ Other recreational items and equipment, } & & & & & \\
\hline & & \multicolumn{2}{|c|}{ gardens and pets } & 12.00 & 15.60 & 15.60 & 21.20 & 10.70 \\
\hline & & 9.3 .1 & Games, toys and hobbies & 2.30 & 2.40 & 2.70 & 2.90 & 1.80 \\
\hline & & 9.3 .2 & Computer software and games & 2.30 & 2.80 & 3.20 & 3.90 & 2.00 \\
\hline & & \multirow[t]{2}{*}{9.3 .3} & Equipment for sport, camping and open-air & & & & & \\
\hline & & & recreation & 1.00 & 1.60 & 1.60 & 2.30 & 0.90 \\
\hline & & 9.3 .4 & Horticultural goods, garden equipment and plants & 2.60 & 4.00 & 3.60 & 5.40 & 2.60 \\
\hline & & 9.3 .5 & Pets and pet food & 3.90 & 4.90 & 4.50 & 6.80 & 3.40 \\
\hline & \multirow[t]{10}{*}{9.4} & \multicolumn{2}{|c|}{ Recreational and cultural services } & 19.30 & 23.70 & 26.40 & 35.20 & 17.30 \\
\hline & & \multirow[t]{2}{*}{9.4 .1} & Sports admissions, subscriptions, leisure class fees & & & & & \\
\hline & & & and equipment hire & 5.00 & 7.10 & 8.60 & 14.70 & 4.80 \\
\hline & & 9.4 .2 & Cinema, theatre and museums etc. & 2.30 & 3.00 & 3.70 & 4.60 & 1.90 \\
\hline & & \multirow[t]{2}{*}{9.4 .3} & TV, video, satellite rental, cable subscriptions, & & & & & \\
\hline & & & TV licences and the Internet & 7.10 & 7.40 & 8.20 & 8.60 & 5.80 \\
\hline & & 9.4 .4 & Miscellaneous entertainments & 1.20 & 1.80 & 1.60 & 3.60 & 1.20 \\
\hline & & \multirow[t]{2}{*}{9.4 .5} & Development of film, deposit for film development, & & & & & \\
\hline & & & passport photos, holiday and school photos & 0.30 & 0.20 & 1.00 & 0.60 & 0.30 \\
\hline & & 9.4 .6 & Gambling payments & 3.40 & 4.20 & 3.30 & 3.10 & 3.20 \\
\hline & \multirow[t]{5}{*}{9.5} & \multicolumn{2}{|c|}{ Newspapers, books and stationery } & 6.50 & 7.20 & 7.90 & 11.30 & 6.00 \\
\hline & & 9.5 .1 & Books & 1.50 & 1.60 & 2.20 & 3.70 & 1.40 \\
\hline & & 9.5 .2 & Diaries, address books, cards etc. & 2.20 & 2.80 & 2.60 & 3.60 & 1.90 \\
\hline & & 9.5 .3 & Newspapers & 1.80 & 1.70 & 1.80 & 2.40 & 1.90 \\
\hline & & 9.5 .4 & Magazines and periodicals & 1.10 & 1.10 & 1.20 & 1.60 & 0.90 \\
\hline & \multirow[t]{3}{*}{9.6} & Packag & holidays & 17.50 & 17.10 & 22.10 & 42.40 & 14.70 \\
\hline & & 9.6 .1 & Package holidays - UK & [1.20] & {$[0.50]$} & {$[1.20]$} & {$[0.90]$} & 1.10 \\
\hline & & 9.6 .2 & Package holidays - abroad & 16.30 & [16.70] & 20.90 & 41.50 & 13.60 \\
\hline 10 & Educa & ation & & 4.80 & 7.30 & 7.10 & 34.60 & 6.20 \\
\hline & 10.1 & Educat & on fees & 4.50 & 7.00 & 6.40 & 33.60 & 5.90 \\
\hline & 10.2 & $\begin{array}{l}\text { Payme } \\
\text { expeno }\end{array}$ & $\begin{array}{l}\text { ts for school trips, other ad-hoc } \\
\text { iture }\end{array}$ & 0.30 & {$[0.30]$} & 0.70 & 1.00 & 0.30 \\
\hline 11 & Rest & taurants & $\&$ hotels & 45.50 & 53.20 & 60.50 & 96.30 & 37.70 \\
\hline & 11.1 & Caterin & g services & 38.00 & 46.10 & 51.00 & 68.70 & 31.00 \\
\hline & & 11.1.1 & Restaurant and café meals & 14.10 & 18.90 & 21.20 & 32.30 & 13.20 \\
\hline & & 11.1.2 & Alcoholic drinks (away from home) & 8.90 & 10.50 & 12.60 & 16.10 & 7.20 \\
\hline & & 11.1.3 & Take away meals eaten at home & 5.00 & 4.90 & 6.00 & 6.60 & 3.90 \\
\hline & & 11.1.4 & Other take-away and snack food & 5.60 & 5.50 & 7.50 & 8.90 & 4.20 \\
\hline & & 11.1.5 & Contract catering (food) and canteens & 4.40 & 6.30 & 3.80 & 4.80 & 2.50 \\
\hline & 11.2 & Accom & nodation services & 7.40 & 7.10 & 9.40 & 27.60 & 6.80 \\
\hline & & 11.2 .1 & Holiday in the UK & 4.00 & 3.20 & 4.10 & 7.70 & 2.80 \\
\hline & & 11.2 .2 & Holiday abroad & 3.40 & 3.80 & 5.30 & 19.70 & 4.00 \\
\hline & & 11.2 .3 & Room hire & [0.10] & {$[0.00]$} & {$[0.00]$} & {$[0.10]$} & {$[0.00]$} \\
\hline
\end{tabular}

Note: The commodity and service categories are not comparable to those in publications before 2001-02.

The numbering system is sequential, it does not use actual COICOP codes.

Please see page xiii for symbols and conventions used in this report. 


\section{Table A8}

Detailed household expenditure by gross income decile group, 2008 (cont.)

based on weighted data and including children's expenditure

\begin{tabular}{|c|c|c|c|c|c|c|c|c|}
\hline & & & $\begin{array}{r}\text { Lowest } \\
\text { ten } \\
\text { per cent }\end{array}$ & $\begin{array}{c}\text { Second } \\
\text { decile } \\
\text { group }\end{array}$ & $\begin{array}{l}\text { Third } \\
\text { decile } \\
\text { group }\end{array}$ & $\begin{array}{l}\text { Fourth } \\
\text { decile } \\
\text { group }\end{array}$ & $\begin{array}{l}\text { Fifth } \\
\text { decile } \\
\text { group }\end{array}$ & $\begin{array}{l}\text { Sixth } \\
\text { decile } \\
\text { group }\end{array}$ \\
\hline \multicolumn{4}{|c|}{ Commodity or service } & \multicolumn{5}{|c|}{ Average weekly household expenditure (f) } \\
\hline \multirow[t]{20}{*}{12} & Misce & ellaneous goods \& services & 10.40 & 15.40 & 19.10 & 21.30 & 28.10 & 33.90 \\
\hline & 12.1 & Personal care & 3.70 & 5.50 & 6.00 & 6.80 & 8.80 & 9.30 \\
\hline & & 12.1.1 Hairdressing, beauty treatment & 1.20 & 1.70 & 1.80 & 2.00 & 2.60 & 2.70 \\
\hline & & 12.1.2 Toilet paper & 0.40 & 0.60 & 0.60 & 0.70 & 0.70 & 0.80 \\
\hline & & 12.1.3 Toiletries and soap & 0.70 & 1.10 & 1.30 & 1.50 & 1.80 & 2.10 \\
\hline & & 12.1.4 Baby toiletries and accessories (disposable) & 0.30 & 0.40 & 0.50 & 0.60 & 0.60 & 0.50 \\
\hline & & 12.1.5 Hair products, cosmetics and & & & & & & \\
\hline & & related electrical appliances & 1.10 & 1.70 & 1.80 & 1.90 & 3.00 & 3.20 \\
\hline & 12.2 & Personal effects & 0.60 & 0.90 & 1.10 & 1.60 & 1.80 & 2.60 \\
\hline & 12.3 & Social protection & [0.60] & [0.70] & 2.20 & {$[0.50]$} & 1.80 & 4.00 \\
\hline & 12.4 & Insurance & 4.70 & 6.30 & 7.60 & 10.00 & 13.40 & 14.70 \\
\hline & & $\begin{array}{l}\text { 12.4.1 Household insurances - structural, contents } \\
\text { and appliances }\end{array}$ & & & & & & \\
\hline & & 12.4.2 Medical insurance premiums & {$[0.30]$} & {$[0.40]$} & 0.70 & 070 & 120 & 120 \\
\hline & & & & & 380 & 530 & 720 & $8>0$ \\
\hline & & 12.4.4 Non-package holiday, other travel insurance & - & - & {$[0.10]$} & {$[0.00]$} & {$[0.20]$} & {$[0.00]$} \\
\hline & 12.5 & Other services n.e.c & 0.80 & 2.00 & 2.20 & 2.50 & 2.40 & 3.20 \\
\hline & & 12.5.1 Moving house & [0.40] & 1.40 & 1.40 & 1.20 & 1.20 & 1.60 \\
\hline & & 12.5.2 Bank, building society, post office, & & & & & & \\
\hline & & credit card charges & 0.10 & 0.20 & 0.10 & 0.30 & 0.50 & 0.40 \\
\hline & & 12.5.3 Other services and professional fees & [0.30] & 0.40 & 0.60 & 0.90 & 0.80 & 1.30 \\
\hline $1-12$ & 2 All $\mathrm{e}$ & expenditure groups & 140.10 & 180.50 & 224.50 & 278.00 & 333.90 & 385.10 \\
\hline \multirow[t]{9}{*}{13} & Other & r expenditure items & 13.70 & 19.50 & 32.60 & 52.10 & 59.10 & 74.10 \\
\hline & 13.1 & Housing: mortgage interest payments, & & & & & & \\
\hline & & council tax etc. & 9.10 & 12.20 & 20.10 & 33.50 & 41.90 & 56.00 \\
\hline & 13.2 & Licences, fines and transfers & 0.80 & 1.30 & 1.60 & 2.30 & 2.80 & 3.40 \\
\hline & 13.3 & Holiday spending & {$[0.60]$} & [1.60] & 5.10 & 6.20 & [3.80] & [5.50] \\
\hline & 13.4 & Money transfers and credit & 3.20 & 4.30 & 5.90 & 10.10 & 10.70 & 9.30 \\
\hline & & 13.4.1 Money, cash gifts given to children & [0.10] & {$[0.00]$} & {$[0.00]$} & {$[0.00]$} & [0.10] & [0.10] \\
\hline & & 13.4.2 Cash gifts and donations & 2.80 & 3.90 & 5.20 & 9.50 & 8.90 & 6.90 \\
\hline & & $\begin{array}{l}\text { 13.4.3 Club instalment payments (child) and interest on } \\
\text { credit cards }\end{array}$ & 0.30 & 0.40 & 0.70 & 0.60 & 1.70 & 2.30 \\
\hline \multicolumn{3}{|c|}{ Total expenditure } & 153.70 & 200.00 & 257.10 & 330.10 & 393.00 & 459.20 \\
\hline \multirow[t]{9}{*}{14} & Other & $r$ items recorded & & & & & & \\
\hline & 14.1 & Life assurance and contributions to pension funds & 0.80 & 1.10 & 2.70 & 4.90 & 9.20 & 14.30 \\
\hline & 14.2 & Other insurance inc. friendly societies & 0.10 & 0.30 & 0.40 & 0.50 & 0.90 & 0.90 \\
\hline & 14.3 & Income tax, payments less refunds & 0.90 & 7.90 & 11.50 & 24.90 & 43.50 & 64.30 \\
\hline & 14.4 & National insurance contributions & {$[0.10]$} & 0.50 & 2.40 & 7.60 & 14.20 & 24.70 \\
\hline & 14.5 & Purchase or alteration of dwellings, mortgages & 3.60 & 6.70 & 14.40 & 16.70 & 24.30 & 36.50 \\
\hline & 14.6 & Savings and investments & 0.40 & 0.40 & 1.00 & 1.40 & 2.50 & 3.00 \\
\hline & 14.7 & Pay off loan to clear other debt & [0.40] & [0.40] & {$[0.80]$} & 1.30 & 1.80 & 3.30 \\
\hline & 14.8 & Windfall receipts from gambling etc ${ }^{3}$ & 0.90 & 2.40 & 1.50 & 0.80 & 2.10 & 2.70 \\
\hline
\end{tabular}

Note: The commodity and service categories are not comparable to those in publications before 2001-02. The numbering system is sequential, it does not use actual COICOP codes.

Please see page xiii for symbols and conventions used in this report.

3 Expressed as an income figure as opposed to an expenditure figure. 


\section{Table A8}

\section{Detailed household expenditure by gross income decile group, 2008 (cont.)}

based on weighted data and including children's expenditure

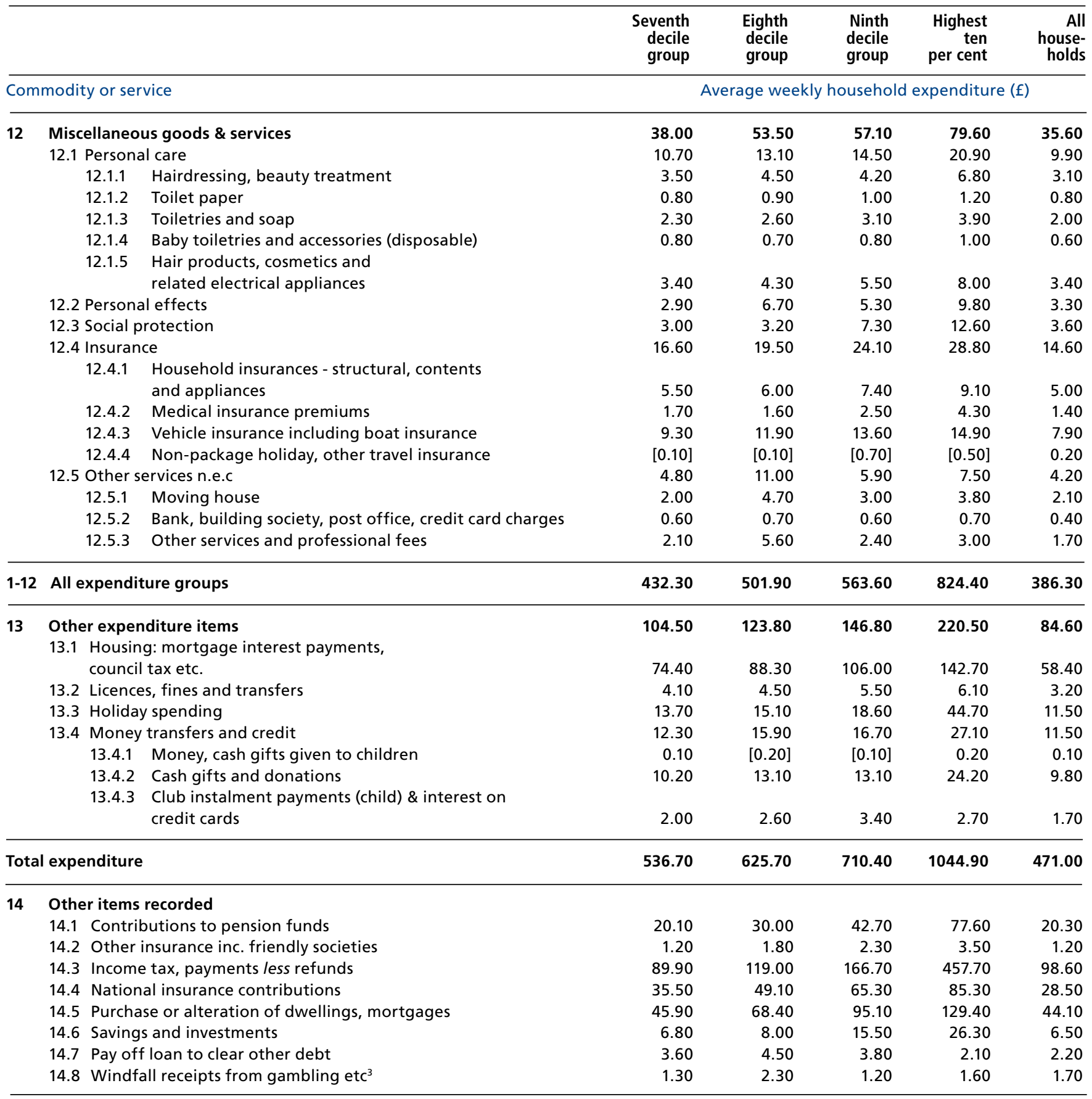

Note: The commodity and service categories are not comparable to those in publications before 2001-02.

The numbering system is sequential, it does not use actual COICOP codes.

Please see page xiii for symbols and conventions used in this report.

3 Expressed as an income figure as opposed to an expenditure figure. 


\section{Table A9}

Household expenditure by disposable income decile group, 2008

based on weighted data and including children's expenditure

\begin{tabular}{|c|c|c|c|c|c|c|c|}
\hline & & $\begin{array}{r}\text { Lowest } \\
\text { ten } \\
\text { per cent }\end{array}$ & $\begin{array}{l}\text { Second } \\
\text { decile } \\
\text { group }\end{array}$ & $\begin{array}{l}\text { Third } \\
\text { decile } \\
\text { group }\end{array}$ & $\begin{array}{l}\text { Fourth } \\
\text { decile } \\
\text { group }\end{array}$ & $\begin{array}{l}\text { Fifth } \\
\text { decile } \\
\text { group }\end{array}$ & $\begin{array}{l}\text { Sixth } \\
\text { decile } \\
\text { group }\end{array}$ \\
\hline \multicolumn{2}{|c|}{ Lower boundary of group ( $\mathrm{f}$ per week) } & & 146 & 224 & 305 & 408 & 522 \\
\hline \multicolumn{2}{|c|}{ Weighted number of households (thousands) } & 2,570 & 2,570 & 2,570 & 2,570 & 2,570 & 2,570 \\
\hline \multicolumn{2}{|c|}{ Total number of households in sample } & 550 & 580 & 600 & 630 & 590 & 600 \\
\hline \multicolumn{2}{|c|}{ Total number of persons in sample } & 710 & 950 & 1,090 & 1,310 & 1,320 & 1,560 \\
\hline \multirow{2}{*}{\multicolumn{2}{|c|}{$\begin{array}{l}\text { Total number of adults in sample } \\
\text { Weighted average number of persons per household }\end{array}$}} & 600 & 770 & 880 & 1,030 & 1,030 & 1,170 \\
\hline & & 1.3 & 1.6 & 1.8 & 2.1 & 2.2 & 2.5 \\
\hline \multicolumn{2}{|c|}{ Commodity or service } & \multicolumn{6}{|c|}{ Average weekly household expenditure $(f)$} \\
\hline 1 & Food \& non-alcoholic drinks & 26.50 & 32.40 & 36.90 & 41.90 & 49.20 & 52.80 \\
\hline 2 & Alcoholic drinks, tobacco \& narcotics & 6.80 & 6.00 & 8.20 & 8.80 & 10.40 & 12.10 \\
\hline 3 & Clothing \& footwear & 6.90 & 8.20 & 10.50 & 13.30 & 17.30 & 19.20 \\
\hline 4 & Housing(net)', fuel \& power & 38.70 & 38.80 & 45.80 & 51.70 & 53.70 & 53.10 \\
\hline 5 & Household goods \& services & 10.20 & 14.30 & 18.60 & 21.10 & 25.20 & 25.60 \\
\hline 6 & Health & 1.30 & 3.00 & 3.30 & 3.80 & 4.60 & 4.80 \\
\hline 7 & Transport & 13.40 & 18.00 & 24.90 & 37.70 & 52.60 & 60.00 \\
\hline 8 & Communication & 6.30 & 7.00 & 7.50 & 9.10 & 11.90 & 12.60 \\
\hline 9 & Recreation \& culture & 16.20 & 23.10 & 30.90 & 38.50 & 48.10 & 68.70 \\
\hline 10 & Education & [1.70] & {$[0.30]$} & 0.50 & 0.70 & 2.70 & 2.80 \\
\hline 11 & Restaurants \& hotels & 9.70 & 12.30 & 15.40 & 22.10 & 27.00 & 35.00 \\
\hline 12 & Miscellaneous goods \& services & 11.50 & 15.00 & 18.50 & 21.00 & 28.40 & 34.70 \\
\hline $1-12$ & All expenditure groups & 149.30 & 178.40 & 221.10 & 269.80 & 331.10 & 381.50 \\
\hline$\underline{13}$ & Other expenditure items & 16.40 & 22.20 & 32.90 & 49.80 & 57.40 & 73.00 \\
\hline \multicolumn{2}{|c|}{ Total expenditure } & 165.70 & 200.60 & 254.00 & 319.60 & 388.50 & 454.40 \\
\hline
\end{tabular}

Note: The commodity and service categories are not comparable to those in publications before 2001-02. Please see page xiii for symbols and conventions used in this report.

1 Excluding mortgage interest payments, council tax and Northern Ireland rates. 


\section{Table A9}

Household expenditure by disposable income decile group 2008, (cont.)

based on weighted data and including children's expenditure

\begin{tabular}{|c|c|c|c|c|c|c|}
\hline & & $\begin{array}{r}\text { Seventh } \\
\text { decile } \\
\text { group }\end{array}$ & $\begin{array}{l}\text { Eighth } \\
\text { decile } \\
\text { group }\end{array}$ & $\begin{array}{l}\text { Ninth } \\
\text { decile } \\
\text { group }\end{array}$ & $\begin{array}{r}\text { Highest } \\
\text { ten } \\
\text { per cent }\end{array}$ & $\begin{array}{r}\text { All } \\
\text { house- } \\
\text { holds }\end{array}$ \\
\hline \multicolumn{2}{|c|}{ Lower boundary of group ( $\mathrm{f}$ per week) } & 664 & 817 & 1026 & 1,356 & \\
\hline \multirow{2}{*}{\multicolumn{2}{|c|}{$\begin{array}{l}\text { Weighted number of households (thousands) } \\
\text { Total number of households in sample }\end{array}$}} & 2,570 & 2,570 & 2,570 & 2,570 & 25,690 \\
\hline & & 590 & 580 & 550 & 580 & 5,850 \\
\hline \multicolumn{2}{|c|}{ Total number of persons in sample } & 1,630 & 1,690 & 1,720 & 1,860 & 13,830 \\
\hline \multirow{2}{*}{\multicolumn{2}{|c|}{$\begin{array}{l}\text { Total number of adults in sample } \\
\text { Weighted average number of persons per household }\end{array}$}} & 1,190 & 1,270 & 1,300 & 1,400 & 10,640 \\
\hline & & 2.8 & 2.9 & 3.1 & 3.2 & 2.4 \\
\hline \multicolumn{2}{|c|}{ Commodity or service } & \multicolumn{5}{|c|}{ Average weekly household expenditure $(f)$} \\
\hline 1 & Food \& non-alcoholic drinks & 55.90 & 63.10 & 69.10 & 79.20 & 50.70 \\
\hline 2 & Alcoholic drinks, tobacco \& narcotics & 11.70 & 14.30 & 13.50 & 16.10 & 10.80 \\
\hline 3 & Clothing \& footwear & 25.40 & 30.00 & 36.50 & 48.60 & 21.60 \\
\hline 4 & Housing(net)' ${ }^{1}$ fuel \& power & 57.80 & 60.50 & 58.40 & 71.80 & 53.00 \\
\hline 5 & Household goods \& services & 34.20 & 43.00 & 43.10 & 66.00 & 30.10 \\
\hline 6 & Health & 5.90 & 6.60 & 6.90 & 11.10 & 5.10 \\
\hline 7 & Transport & 71.30 & 82.90 & 114.20 & 159.10 & 63.40 \\
\hline 8 & Communication & 14.00 & 14.80 & 17.00 & 19.30 & 12.00 \\
\hline 9 & Recreation \& culture & 69.00 & 75.90 & 89.80 & 140.40 & 60.10 \\
\hline 10 & Education & 4.60 & 5.10 & 10.80 & 32.60 & 6.20 \\
\hline 11 & Restaurants \& hotels & 42.40 & 54.60 & 61.80 & 97.00 & 37.70 \\
\hline 12 & Miscellaneous goods \& services & 38.10 & 51.50 & 57.10 & 80.50 & 35.60 \\
\hline $1-12$ & All expenditure groups & 430.50 & 502.30 & 578.10 & 821.70 & 386.30 \\
\hline 13 & Other expenditure items & 106.60 & 125.20 & 142.40 & 220.60 & 84.60 \\
\hline \multicolumn{2}{|c|}{ Total expenditure } & 537.00 & 627.60 & 720.50 & 1042.30 & 471.00 \\
\hline \multicolumn{7}{|c|}{ Average weekly expenditure per person (f) } \\
\hline
\end{tabular}

Note: The commodity and service categories are not comparable to those in publications before 2001-02. Please see page xiii for symbols and conventions used in this report.

1 Excluding mortgage interest payments, council tax and Northern Ireland rates. 


\section{Table A10}

\section{Household expenditure as a percentage of total expenditure by disposable income} decile group, 2008

based on weighted data and including children's expenditure

\begin{tabular}{|c|c|c|c|c|c|c|c|}
\hline & & $\begin{array}{r}\text { Lowest } \\
\text { ten } \\
\text { per cent }\end{array}$ & $\begin{array}{l}\text { Second } \\
\text { decile } \\
\text { group }\end{array}$ & $\begin{array}{l}\text { Third } \\
\text { decile } \\
\text { group }\end{array}$ & $\begin{array}{l}\text { Fourth } \\
\text { decile } \\
\text { group }\end{array}$ & $\begin{array}{l}\text { Fifth } \\
\text { decile } \\
\text { group }\end{array}$ & $\begin{array}{l}\text { Sixth } \\
\text { decile } \\
\text { group }\end{array}$ \\
\hline \multicolumn{2}{|c|}{ Lower boundary of group ( $\mathrm{f}$ per week) } & & 146 & 224 & 305 & 408 & 522 \\
\hline \multirow{5}{*}{\multicolumn{2}{|c|}{$\begin{array}{l}\text { Weighted number of households (thousands) } \\
\text { Total number of households in sample } \\
\text { Total number of persons in sample } \\
\text { Total number of adults in sample } \\
\text { Weighted average number of persons per household }\end{array}$}} & 2,570 & 2,570 & 2,570 & 2,570 & 2,570 & 2,570 \\
\hline & & 550 & 580 & 600 & 630 & 590 & 600 \\
\hline & & 710 & 950 & 1,090 & 1,310 & 1,320 & 1,560 \\
\hline & & 600 & 770 & 880 & 1,030 & 1,030 & 1,170 \\
\hline & & 1.3 & 1.6 & 1.8 & 2.1 & 2.2 & 2.5 \\
\hline \multicolumn{2}{|c|}{ Commodity or service } & \multicolumn{6}{|c|}{ Percentage of total expenditure } \\
\hline 1 & Food \& non-alcoholic drinks & 16 & 16 & 15 & 13 & 13 & 12 \\
\hline 2 & Alcoholic drinks, tobacco \& narcotics & 4 & 3 & 3 & 3 & 3 & 3 \\
\hline 3 & Clothing \& footwear & 4 & 4 & 4 & 4 & 4 & 4 \\
\hline 4 & Housing(net)', fuel \& power & 23 & 19 & 18 & 16 & 14 & 12 \\
\hline 5 & Household goods \& services & 6 & 7 & 7 & 7 & 6 & 6 \\
\hline 6 & Health & 1 & 1 & 1 & 1 & 1 & 1 \\
\hline 7 & Transport & 8 & 9 & 10 & 12 & 14 & 13 \\
\hline 8 & Communication & 4 & 4 & 3 & 3 & 3 & 3 \\
\hline 9 & Recreation \& culture & 10 & 12 & 12 & 12 & 12 & 15 \\
\hline 10 & Education & [1] & [0] & 0 & 0 & 1 & 1 \\
\hline 11 & Restaurants \& hotels & 6 & 6 & 6 & 7 & 7 & 8 \\
\hline 12 & Miscellaneous goods \& services & 7 & 7 & 7 & 7 & 7 & 8 \\
\hline $1-12$ & All expenditure groups & 90 & 89 & 87 & 84 & 85 & 84 \\
\hline 13 & Other expenditure items & 10 & 11 & 13 & 16 & 15 & 16 \\
\hline \multicolumn{2}{|c|}{ Total expenditure } & 100 & 100 & 100 & 100 & 100 & 100 \\
\hline
\end{tabular}

Note: The commodity and service categories are not comparable to those in publications before 2001-02.

Please see page xiii for symbols and conventions used in this report.

1 Excluding mortgage interest payments, council tax and Northern Ireland rates. 


\section{Table A10}

\section{Household expenditure as a percentage of total expenditure by disposable income} decile group, 2008 (cont.)

based on weighted data and including children's expenditure

\begin{tabular}{|c|c|c|c|c|c|}
\hline & $\begin{array}{r}\text { Seventh } \\
\text { decile } \\
\text { group }\end{array}$ & $\begin{array}{l}\text { Eighth } \\
\text { decile } \\
\text { group }\end{array}$ & $\begin{array}{l}\text { Ninth } \\
\text { decile } \\
\text { group }\end{array}$ & $\begin{array}{r}\text { Highest } \\
\text { ten } \\
\text { per cent }\end{array}$ & $\begin{array}{r}\text { All } \\
\text { house- } \\
\text { holds }\end{array}$ \\
\hline Lower boundary of group ( $\mathrm{f}$ per week) & 664 & 817 & 1026 & 1,356 & \\
\hline Weighted number of households (thousands) & 2,570 & 2,570 & 2,570 & 2,570 & 25,690 \\
\hline Total number of households in sample & 590 & 580 & 550 & 580 & 5,850 \\
\hline Total number of persons in sample & 1,630 & 1,690 & 1,720 & 1,860 & 13,830 \\
\hline Total number of adults in sample & 1,190 & 1,270 & 1,300 & 1,400 & 10,640 \\
\hline Weighted average number of persons per household & 2.8 & 2.9 & 3.1 & 3.2 & 2.4 \\
\hline
\end{tabular}

\begin{tabular}{|c|c|c|c|c|c|c|}
\hline 1 & Food \& non-alcoholic drinks & 10 & 10 & 10 & 8 & 11 \\
\hline 2 & Alcoholic drinks, tobacco \& narcotics & 2 & 2 & 2 & 2 & 2 \\
\hline 3 & Clothing \& footwear & 5 & 5 & 5 & 5 & 5 \\
\hline 4 & Housing(net) ${ }^{1}$, fuel \& power & 11 & 10 & 8 & 7 & 11 \\
\hline 5 & Household goods \& services & 6 & 7 & 6 & 6 & 6 \\
\hline 6 & Health & 1 & 1 & 1 & 1 & 1 \\
\hline 7 & Transport & 13 & 13 & 16 & 15 & 13 \\
\hline 8 & Communication & 3 & 2 & 2 & 2 & 3 \\
\hline 9 & Recreation \& culture & 13 & 12 & 12 & 13 & 13 \\
\hline 10 & Education & 1 & 1 & 1 & 3 & 1 \\
\hline 11 & Restaurants \& hotels & 8 & 9 & 9 & 9 & 8 \\
\hline 12 & Miscellaneous goods \& services & 7 & 8 & 8 & 8 & 8 \\
\hline $1-12$ & All expenditure groups & 80 & 80 & 80 & 79 & 82 \\
\hline 13 & Other expenditure items & 20 & 20 & 20 & 21 & 18 \\
\hline \multicolumn{2}{|c|}{ Total expenditure } & 100 & 100 & 100 & 100 & 100 \\
\hline
\end{tabular}

Note: The commodity and service categories are not comparable to those in publications before 2001-02. Please see page xiii for symbols and conventions used in this report.

1 Excluding mortgage interest payments, council tax and Northern Ireland rates. 


\section{Table A11}

Household expenditure by age of household reference person, 2008

based on weighted data and including children's expenditure

\begin{tabular}{|c|c|c|c|c|c|c|c|}
\hline & & $\begin{array}{r}\text { Less } \\
\text { than } \\
30\end{array}$ & $\begin{array}{l}30 \\
\text { to } \\
49\end{array}$ & $\begin{array}{l}50 \\
\text { to } \\
64\end{array}$ & $\begin{array}{l}65 \\
\text { to } \\
74\end{array}$ & $\begin{array}{r}75 \\
\text { or } \\
\text { over }\end{array}$ & $\begin{array}{r}\text { All } \\
\text { house- } \\
\text { holds }\end{array}$ \\
\hline \multicolumn{2}{|c|}{ Weighted number of households (thousands) } & 2,530 & 9,740 & 6,750 & 3,140 & 3,520 & 25,690 \\
\hline \multicolumn{2}{|c|}{ Total number of households in sample } & 460 & 2,230 & 1,590 & 810 & 760 & 5,850 \\
\hline \multicolumn{2}{|c|}{ Total number of persons in sample } & 1,120 & 6,710 & 3,480 & 1,410 & 1,120 & 13,830 \\
\hline \multicolumn{2}{|c|}{ Total number of adults in sample } & 800 & 4,170 & 3,160 & 1,390 & 1,110 & 10,640 \\
\hline \multicolumn{2}{|c|}{ Weighted average number of persons per household } & 2.4 & 3.0 & 2.3 & 1.8 & 1.4 & 2.4 \\
\hline \multicolumn{2}{|c|}{ Commodity or service } & \multicolumn{6}{|c|}{ Average weekly household expenditure $(f)$} \\
\hline 1 & Food \& non-alcoholic drinks & 38.70 & 57.00 & 55.50 & 48.20 & 34.90 & 50.70 \\
\hline 2 & Alcoholic drinks, tobacco \& narcotics & 10.50 & 12.30 & 12.80 & 9.30 & 4.30 & 10.80 \\
\hline 3 & Clothing \& footwear & 23.20 & 27.50 & 23.70 & 12.70 & 7.70 & 21.60 \\
\hline 4 & Housing(net)' ${ }^{1}$, fuel \& power & 76.20 & 59.50 & 49.10 & 42.00 & 36.10 & 53.00 \\
\hline 5 & Household goods \& services & 23.90 & 35.60 & 32.90 & 26.20 & 17.60 & 30.10 \\
\hline 6 & Health & 2.80 & 4.20 & 7.70 & 4.50 & 4.90 & 5.10 \\
\hline 7 & Transport & 55.80 & 77.90 & 80.30 & 41.20 & 16.00 & 63.40 \\
\hline 8 & Communication & 13.20 & 14.10 & 13.20 & 8.20 & 5.90 & 12.00 \\
\hline 9 & Recreation \& culture & 41.40 & 67.20 & 70.90 & 63.70 & 29.60 & 60.10 \\
\hline 10 & Education & 3.00 & 8.90 & 8.80 & [1.30] & [0.40] & 6.20 \\
\hline 11 & Restaurants \& hotels & 38.20 & 46.40 & 44.00 & 25.10 & 12.60 & 37.70 \\
\hline 12 & Miscellaneous goods \& services & 31.30 & 45.40 & 36.60 & 24.00 & 20.10 & 35.60 \\
\hline $1-12$ & All expenditure groups & 358.20 & 456.20 & 435.60 & 306.50 & 190.10 & 386.30 \\
\hline 13 & Other expenditure items & 83.00 & 125.70 & 73.60 & 47.20 & 26.80 & 84.60 \\
\hline \multicolumn{2}{|c|}{ Total expenditure } & 441.20 & 581.90 & 509.20 & 353.60 & 216.80 & 471.00 \\
\hline \multirow{2}{*}{\multicolumn{2}{|c|}{$\begin{array}{l}\text { Average weekly expenditure per person }(f) \\
\text { Total expenditure }\end{array}$}} & & & & & & \\
\hline & & 183.00 & 197.20 & 226.00 & 200.30 & 154.20 & 199.80 \\
\hline
\end{tabular}

Note: The commodity and service categories are not comparable to those in publications before 2001-02. Please see page xiii for symbols and conventions used in this report.

1 Excluding mortgage interest payments, council tax and Northern Ireland rates. 


\section{Table A12}

\section{Household expenditure as a percentage of total expenditure}

by age of household reference person, 2008

based on weighted data and including children's expenditure

\begin{tabular}{|c|c|c|c|c|c|c|c|}
\hline & & $\begin{array}{r}\text { Less } \\
\text { than } \\
30\end{array}$ & $\begin{array}{l}30 \\
\text { to } \\
49\end{array}$ & $\begin{array}{l}50 \\
\text { to } \\
64\end{array}$ & $\begin{array}{l}65 \\
\text { to } \\
74\end{array}$ & $\begin{array}{r}75 \\
\text { or } \\
\text { over }\end{array}$ & $\begin{array}{r}\text { All } \\
\text { house- } \\
\text { holds }\end{array}$ \\
\hline \multicolumn{2}{|c|}{ Weighted number of households (thousands) } & 2,530 & 9,740 & 6,750 & 3,140 & 3,520 & 25,690 \\
\hline \multicolumn{2}{|c|}{ Total number of households in sample } & 460 & 2,230 & 1,590 & 810 & 760 & 5,850 \\
\hline \multicolumn{2}{|c|}{ Total number of persons in sample } & 1,120 & 6,710 & 3,480 & 1,410 & 1,120 & 13,830 \\
\hline \multicolumn{2}{|c|}{ Total number of adults in sample } & 800 & 4,170 & 3,160 & 1,390 & 1,110 & 10,640 \\
\hline \multicolumn{2}{|c|}{ Weighted average number of persons per household } & 2.4 & 3.0 & 2.3 & 1.8 & 1.4 & 2.4 \\
\hline \multicolumn{3}{|c|}{ Commodity or service } & \multicolumn{4}{|c|}{ Percentage of total expenditure } & \\
\hline 1 & Food \& non-alcoholic drinks & 9 & 10 & 11 & 14 & 16 & 11 \\
\hline 2 & Alcoholic drinks, tobacco \& narcotics & 2 & 2 & 3 & 3 & 2 & 2 \\
\hline 3 & Clothing \& footwear & 5 & 5 & 5 & 4 & 4 & 5 \\
\hline 4 & Housing(net) ${ }^{1}$, fuel \& power & 17 & 10 & 10 & 12 & 17 & 11 \\
\hline 6 & Health & 1 & 1 & 2 & 1 & 2 & 1 \\
\hline 7 & Transport & 13 & 13 & 16 & 12 & 7 & 13 \\
\hline 8 & Communication & 3 & 2 & 3 & 2 & 3 & 3 \\
\hline 9 & Recreation \& culture & 9 & 12 & 14 & 18 & 14 & 13 \\
\hline 10 & Education & 1 & 2 & 2 & [0] & [0] & 1 \\
\hline 11 & Restaurants \& hotels & 9 & 8 & 9 & 7 & 6 & 8 \\
\hline 12 & Miscellaneous goods \& services & 7 & 8 & 7 & 7 & 9 & 8 \\
\hline $1-12$ & All expenditure groups & 81 & 78 & 86 & 87 & 88 & 82 \\
\hline 13 & Other expenditure items & 19 & 22 & 14 & 13 & 12 & 18 \\
\hline
\end{tabular}

Note: The commodity and service categories are not comparable to those in publications before 2001-02. Please see page xiii for symbols and conventions used in this report.

1 Excluding mortgage interest payments, council tax and Northern Ireland rates. 


\section{Table A13}

\section{Detailed household expenditure by age of household reference person, 2008}

based on weighted data and including children's expenditure

\begin{tabular}{|c|c|c|c|c|c|c|c|c|}
\hline & & & $\begin{array}{l}\text { Less } \\
\text { than } \\
30\end{array}$ & $\begin{array}{r}30 \\
\text { to } \\
49\end{array}$ & $\begin{array}{l}50 \\
\text { to } \\
64\end{array}$ & $\begin{array}{l}65 \\
\text { to } \\
74\end{array}$ & $\begin{array}{r}75 \\
\text { or } \\
\text { over }\end{array}$ & $\begin{array}{r}\text { All } \\
\text { house- } \\
\text { holds }\end{array}$ \\
\hline Weigh & numb & r of households (thousands) & 2,530 & 9,740 & 6,750 & 3,140 & 3,520 & 25,690 \\
\hline Total & nber of & households in sample & 460 & 2,230 & 1,590 & 810 & 760 & 5,850 \\
\hline Total & nber of & persons in sample & 1,120 & 6,700 & 3,480 & 1,410 & 1,120 & 13,830 \\
\hline Total & nber of & adults in sample & 810 & 4,170 & 3,160 & 1,390 & 1,110 & 10,640 \\
\hline Weigh & avera & e number of persons per household & 2.4 & 3.0 & 2.3 & 1.8 & 1.4 & 2.4 \\
\hline Comm & ity or s & rvice & & rage we & louseho & penditu & & \\
\hline $1 \mathrm{Fc}$ & \& non & alcoholic drinks & 38.70 & $\mathbf{5 7 . 0 0}$ & 55.50 & 48.20 & 34.90 & 50.70 \\
\hline 1. & Food & & 35.00 & 52.00 & 51.30 & 45.00 & 32.60 & 46.70 \\
\hline & 1.1 .1 & Bread, rice and cereals & 4.10 & 5.80 & 5.00 & 4.10 & 3.10 & 4.90 \\
\hline & 1.1 .2 & Pasta products & 0.50 & 0.60 & 0.30 & 0.20 & 0.10 & 0.40 \\
\hline & 1.1 .3 & Buns, cakes, biscuits etc. & 2.20 & 3.40 & 3.40 & 3.30 & 2.80 & 3.20 \\
\hline & 1.1 .4 & Pastry (savoury) & 0.80 & 0.90 & 0.60 & 0.40 & 0.20 & 0.70 \\
\hline & 1.1 .5 & Beef (fresh, chilled or frozen) & 0.90 & 1.70 & 2.00 & 1.50 & 1.00 & 1.60 \\
\hline & 1.1 .6 & Pork (fresh, chilled or frozen) & 0.40 & 0.60 & 0.90 & 0.70 & 0.40 & 0.60 \\
\hline & 1.1 .7 & Lamb (fresh, chilled or frozen) & 0.30 & 0.60 & 0.80 & 0.80 & 0.60 & 0.60 \\
\hline & 1.1 .8 & Poultry (fresh, chilled or frozen) & 1.30 & 2.20 & 2.10 & 1.90 & 0.90 & 1.90 \\
\hline & 1.1 .9 & Bacon and ham & 0.50 & 0.90 & 1.10 & 1.10 & 0.80 & 0.90 \\
\hline & 1.1 .10 & Other meat and meat preparations & 4.00 & 5.70 & 5.80 & 4.70 & 3.70 & 5.20 \\
\hline & 1.1 .11 & Fish and fish products & 1.30 & 2.20 & 2.60 & 2.60 & 2.20 & 2.30 \\
\hline & 1.1 .12 & Milk & 2.10 & 3.00 & 2.70 & 2.50 & 2.10 & 2.60 \\
\hline & 1.1 .13 & Cheese and curd & 1.40 & 1.90 & 1.90 & 1.60 & 0.90 & 1.70 \\
\hline & 1.1 .14 & Eggs & 0.50 & 0.70 & 0.70 & 0.60 & 0.50 & 0.60 \\
\hline & 1.1.15 & Other milk products & 1.40 & 2.10 & 2.10 & 1.70 & 1.30 & 1.90 \\
\hline & 1.1.16 & Butter & 0.20 & 0.30 & 0.40 & 0.50 & 0.40 & 0.40 \\
\hline & 1.1.17 & Margarine, other vegetable fats and peanut butter & 0.30 & 0.50 & 0.60 & 0.60 & 0.50 & 0.50 \\
\hline & 1.1.18 & Cooking oils and fats & 0.20 & 0.30 & 0.30 & 0.40 & 0.20 & 0.30 \\
\hline & 1.1 .19 & Fresh fruit & 1.80 & 3.10 & 3.30 & 3.40 & 2.40 & 3.00 \\
\hline & 1.1 .20 & Other fresh, chilled or frozen fruits & 0.20 & 0.40 & 0.40 & 0.30 & 0.20 & 0.40 \\
\hline & 1.1 .21 & Dried fruit and nuts & 0.20 & 0.50 & 0.60 & 0.70 & 0.40 & 0.50 \\
\hline & 1.1 .22 & Preserved fruit and fruit based products & 0.10 & 0.10 & 0.10 & 0.20 & 0.20 & 0.10 \\
\hline & 1.1 .23 & Fresh vegetables & 2.60 & 4.10 & 4.30 & 3.70 & 2.40 & 3.70 \\
\hline & 1.1 .24 & Dried vegetables & [0.00] & 0.10 & 0.00 & 0.10 & 0.00 & 0.00 \\
\hline & 1.1 .25 & Other preserved or processed vegetables & 1.00 & 1.40 & 1.30 & 0.90 & 0.50 & 1.20 \\
\hline & 1.1 .26 & Potatoes & 0.60 & 0.90 & 1.00 & 1.00 & 0.70 & 0.90 \\
\hline & 1.1 .27 & Other tubers and products of tuber vegetables & 1.20 & 1.60 & 1.30 & 0.90 & 0.60 & 1.30 \\
\hline & 1.1 .28 & Sugar and sugar products & 0.20 & 0.40 & 0.30 & 0.30 & 0.30 & 0.30 \\
\hline & 1.1 .29 & Jams, marmalades & 0.10 & 0.20 & 0.20 & 0.40 & 0.40 & 0.30 \\
\hline & 1.1 .30 & Chocolate & 1.10 & 1.70 & 1.60 & 1.20 & 1.10 & 1.50 \\
\hline & 1.1 .31 & Confectionery products & 0.40 & 0.70 & 0.60 & 0.50 & 0.40 & 0.60 \\
\hline & 1.1 .32 & Edible ices and ice cream & 0.40 & 0.60 & 0.50 & 0.40 & 0.30 & 0.50 \\
\hline & 1.1 .33 & Other food products & 2.40 & 2.70 & 2.30 & 1.80 & 1.20 & 2.30 \\
\hline 1.2 & Non-a & Icoholic drinks & 3.60 & 5.00 & 4.20 & 3.20 & 2.20 & 4.00 \\
\hline & 1.2 .1 & Coffee & 0.30 & 0.60 & 0.70 & 0.60 & 0.40 & 0.60 \\
\hline & 1.2 .2 & Tea & 0.20 & 0.40 & 0.50 & 0.50 & 0.50 & 0.40 \\
\hline & 1.2 .3 & Cocoa and powdered chocolate & 0.10 & 0.10 & 0.10 & 0.10 & 0.10 & 0.10 \\
\hline & 1.2 .4 & Fruit and vegetable juices (inc. fruit squash) & 1.10 & 1.50 & 1.10 & 0.80 & 0.50 & 1.10 \\
\hline & 1.2 .5 & Mineral or spring waters & 0.20 & 0.30 & 0.20 & 0.20 & 0.10 & 0.20 \\
\hline & 1.2 .6 & Soft drinks (inc. fizzy and ready & & & & & & \\
\hline & & to drink fruit drinks) & 1.80 & 2.20 & 1.50 & 1.00 & 0.60 & 1.60 \\
\hline
\end{tabular}

Note: The commodity and service categories are not comparable to those in publications before 2001-02. The numbering system is sequential, it does not use actual COICOP codes. Please see page xiii for symbols and conventions used in this report. 


\section{Table A13}

Detailed household expenditure by age of household reference person, 2008 (cont.) based on weighted data and including children's expenditure

\begin{tabular}{|c|c|c|c|c|c|c|c|c|c|}
\hline & & & & $\begin{array}{r}\text { Less } \\
\text { than } \\
30\end{array}$ & $\begin{array}{l}30 \\
\text { to } \\
49\end{array}$ & $\begin{array}{l}50 \\
\text { to } \\
64\end{array}$ & $\begin{array}{l}65 \\
\text { to } \\
74\end{array}$ & $\begin{array}{r}75 \\
\text { or } \\
\text { over }\end{array}$ & $\begin{array}{r}\text { All } \\
\text { house- } \\
\text { holds }\end{array}$ \\
\hline \multicolumn{5}{|c|}{ Commodity or service } & \multicolumn{5}{|c|}{ Average weekly household expenditure (f) } \\
\hline \multirow[t]{9}{*}{2} & \multicolumn{3}{|c|}{ Alcoholic drink, tobacco \& narcotics } & 10.50 & 12.30 & 12.80 & 9.30 & 4.30 & 10.80 \\
\hline & \multirow[t]{5}{*}{2.1} & \multicolumn{2}{|c|}{ Alcoholic drinks } & 4.90 & 7.00 & 7.50 & 5.80 & 3.00 & 6.20 \\
\hline & & 2.1.1 & Spirits and liqueurs (brought home) & 0.90 & 0.90 & 1.50 & 1.50 & 1.20 & 1.20 \\
\hline & & 2.1 .2 & Wines, fortified wines (brought home) & 1.90 & 3.70 & 4.10 & 3.30 & 1.40 & 3.30 \\
\hline & & 2.1 .3 & Beer, lager, ciders and perry (brought home) & 1.90 & 2.30 & 1.80 & 1.00 & 0.40 & 1.70 \\
\hline & & 2.1 .4 & Alcopops (brought home) & 0.20 & 0.10 & 0.10 & {$[0.00]$} & {$[0.00]$} & 0.10 \\
\hline & \multirow[t]{3}{*}{2.2} & \multicolumn{2}{|c|}{ Tobacco and narcotics } & 5.60 & 5.30 & 5.40 & 3.50 & 1.30 & 4.60 \\
\hline & & 2.2 .1 & Cigarettes & 4.80 & 4.60 & 4.60 & 2.90 & 1.00 & 3.90 \\
\hline & & 2.2 .2 & Cigars, other tobacco products and narcotics & 0.90 & 0.70 & 0.80 & 0.50 & {$[0.30]$} & 0.70 \\
\hline \multirow[t]{14}{*}{3} & \multicolumn{3}{|c|}{ Clothing \& footwear } & 23.20 & 27.50 & 23.70 & 12.70 & 7.70 & 21.60 \\
\hline & \multirow[t]{12}{*}{3.1} & \multicolumn{2}{|c|}{ Clothing } & 18.70 & 22.10 & 19.40 & 10.80 & 6.00 & 17.50 \\
\hline & & 3.1.1 & Men's outer garments & 6.20 & 5.60 & 5.10 & 2.60 & 1.10 & 4.50 \\
\hline & & 3.1 .2 & Men's under garments & 0.30 & 0.40 & 0.40 & 0.30 & 0.20 & 0.30 \\
\hline & & 3.1 .3 & Women's outer garments & 7.10 & 8.80 & 9.80 & 4.80 & 3.30 & 7.60 \\
\hline & & 3.1 .4 & Women's under garments & 1.00 & 1.20 & 1.20 & 0.80 & 0.60 & 1.10 \\
\hline & & 3.1.5 & Boys' outer garments (5-15) & 0.40 & 1.50 & 0.40 & {$[0.20]$} & [0.10] & 0.80 \\
\hline & & 3.1.6 & Girls' outer garments (5-15) & 0.50 & 1.80 & 0.60 & 0.40 & {$[0.10]$} & 1.00 \\
\hline & & 3.1.7 & Infants' outer garments (under 5) & 1.10 & 1.00 & 0.40 & 0.30 & {$[0.10]$} & 0.60 \\
\hline & & 3.1.8 & Children's under garments (under 16) & 0.40 & 0.50 & 0.20 & 0.10 & {$[0.00]$} & 0.30 \\
\hline & & 3.1 .9 & Accessories & 0.80 & 0.80 & 0.80 & 0.50 & 0.30 & 0.70 \\
\hline & & \multicolumn{2}{|c|}{$\begin{array}{l}\text { 3.1.10 Haberdashery, } \\
\text { clothing hire }\end{array}$} & {$[0.60]$} & 0.20 & 0.20 & 0.40 & 0.20 & 0.30 \\
\hline & & 3.1.11 & Dry cleaners, laundry and dyeing & {$[0.20]$} & 0.20 & 0.40 & 0.30 & 0.20 & 0.30 \\
\hline & 3.2 & \multicolumn{2}{|c|}{ Footwear } & 4.50 & 5.40 & 4.40 & 2.00 & 1.70 & 4.10 \\
\hline \multirow[t]{13}{*}{4} & \multicolumn{3}{|c|}{ Housing (net)', fuel \& power } & 76.10 & 59.50 & 49.10 & 42.00 & 36.10 & 53.00 \\
\hline & \multirow[t]{5}{*}{4.1} & \multicolumn{2}{|c|}{ Actual rentals for housing } & 70.80 & 35.50 & 21.20 & 21.50 & 21.70 & 31.60 \\
\hline & & 4.1.1 & Gross rent & 70.80 & 35.50 & 20.90 & 21.50 & 21.70 & 31.50 \\
\hline & & 4.1.2 & less housing benefit, rebates \& allowances rec'd & 19.20 & 11.40 & 9.40 & 11.60 & 14.70 & 12.10 \\
\hline & & 4.1 .3 & Net rent ${ }^{2}$ & 51.60 & 24.00 & 11.50 & 9.90 & 7.10 & 19.40 \\
\hline & & 4.1.4 & Second dwelling rent & - & {$[0.00]$} & [0.30] & - & - & {$[0.10]$} \\
\hline & 4.2 & Maint & enance and repair of dwelling & 3.10 & 8.10 & 8.80 & 6.70 & 5.00 & 7.20 \\
\hline & \multirow[t]{2}{*}{4.3} & \multirow{2}{*}{\multicolumn{2}{|c|}{$\begin{array}{l}\text { Water supply and miscellaneous services } \\
\text { relating to the dwelling }\end{array}$}} & & & & & & \\
\hline & & & & 6.90 & 7.50 & 7.30 & 7.50 & 7.70 & 7.40 \\
\hline & \multirow[t]{4}{*}{4.4} & \multicolumn{2}{|c|}{ Electricity, gas and other fuels } & 14.50 & 19.90 & 21.10 & 17.90 & 16.40 & 18.90 \\
\hline & & 4.4.1 & Electricity & 7.60 & 9.50 & 10.00 & 8.30 & 7.60 & 9.00 \\
\hline & & 4.4.2 & Gas & 6.40 & 8.80 & 8.90 & 7.50 & 7.10 & 8.20 \\
\hline & & 4.4.3 & Other fuels & 0.60 & 1.60 & 2.20 & 2.10 & 1.60 & 1.70 \\
\hline
\end{tabular}

Note: The commodity and service categories are not comparable to those in publications before 2001-02.

The numbering system is sequential, it does not use actual COICOP codes.

Please see page xiii for symbols and conventions used in this report.

1 Excluding mortgage interest payments, council tax and Northern Ireland rates.

2 The figure included in total expenditure is net rent as opposed to gross rent 


\section{Table A13}

Detailed household expenditure by age of household reference person, 2008 (cont.) based on weighted data and including children's expenditure

\begin{tabular}{|c|c|c|c|c|c|c|c|c|c|}
\hline & & & & $\begin{array}{r}\text { Less } \\
\text { than } \\
30\end{array}$ & $\begin{array}{l}30 \\
\text { to } \\
49\end{array}$ & $\begin{array}{l}50 \\
\text { to } \\
64\end{array}$ & $\begin{array}{l}65 \\
\text { to } \\
74\end{array}$ & $\begin{array}{r}75 \\
\text { or } \\
\text { over }\end{array}$ & $\begin{array}{r}\text { All } \\
\text { house- } \\
\text { holds }\end{array}$ \\
\hline \multicolumn{5}{|c|}{ Commodity or service } & \multicolumn{5}{|c|}{ Average weekly household expenditure (f) } \\
\hline \multirow[t]{14}{*}{5} & \multicolumn{3}{|c|}{ Household goods \& services } & 23.90 & 35.70 & 32.90 & 26.20 & 17.60 & 30.10 \\
\hline & \multirow[t]{3}{*}{5.1} & \multicolumn{2}{|c|}{ Furniture and furnishings and floor coverings } & 16.20 & 20.80 & 17.50 & 13.50 & 6.40 & 16.60 \\
\hline & & 5.1 .1 & Furniture and furnishings & 13.10 & 16.50 & 13.70 & 9.40 & 5.00 & 13.00 \\
\hline & & 5.1 .2 & Floor coverings & 3.20 & 4.40 & 3.80 & 4.20 & 1.40 & 3.70 \\
\hline & 5.2 & \multicolumn{2}{|c|}{ Household textiles } & 1.00 & 1.80 & 1.80 & 1.70 & 0.50 & 1.50 \\
\hline & 5.3 & \multicolumn{2}{|c|}{ Household appliances } & 2.00 & 4.30 & 3.80 & 2.00 & 2.90 & 3.50 \\
\hline & 5.4 & \multicolumn{2}{|c|}{ Glassware, tableware and household utensils } & 1.20 & 1.40 & 1.50 & 1.60 & 0.70 & 1.30 \\
\hline & 5.5 & \multicolumn{2}{|c|}{ Tools and equipment for house and garden } & 0.90 & 2.40 & 2.70 & 2.40 & 0.70 & 2.10 \\
\hline & 5.6 & \multirow{2}{*}{\multicolumn{2}{|c|}{ maintenance }} & & & & & & \\
\hline & & & & 2.50 & 4.90 & 5.60 & 5.00 & 6.30 & 5.10 \\
\hline & & 5.6.1 & Cleaning materials & 1.50 & 2.40 & 2.50 & 2.20 & 1.40 & 2.20 \\
\hline & & 5.6 .2 & Household goods and hardware & 0.60 & 1.20 & 1.30 & 1.10 & 0.60 & 1.10 \\
\hline & & 5.6 .3 & Domestic services, carpet cleaning, & & & & & & \\
\hline & & & hire of furniture/furnishings & 0.30 & 1.30 & 1.80 & 1.70 & 4.30 & 1.80 \\
\hline \multirow[t]{5}{*}{6} & \multicolumn{3}{|c|}{ Health } & 2.80 & 4.20 & 7.70 & 4.50 & 4.90 & 5.10 \\
\hline & 6.1 & \multicolumn{2}{|c|}{ Medical products, appliances and equipment } & 2.20 & 2.60 & 3.90 & 2.80 & 3.70 & 3.10 \\
\hline & & 6.1 .1 & Medicines, prescriptions and healthcare products & 0.90 & 1.60 & 2.20 & 1.80 & 1.40 & 1.70 \\
\hline & & 6.1 .2 & Spectacles, lenses, accessories and repairs & 1.30 & 1.00 & 1.80 & 1.10 & 2.30 & 1.40 \\
\hline & 6.2 & \multicolumn{2}{|c|}{ Hospital services } & {$[0.60]$} & 1.60 & 3.80 & 1.70 & 1.20 & 2.00 \\
\hline \multirow[t]{15}{*}{7} & \multicolumn{3}{|c|}{ Transport } & 55.80 & 77.90 & 80.30 & 41.20 & 16.00 & 63.40 \\
\hline & \multirow[t]{4}{*}{7.1} & \multicolumn{2}{|c|}{ Purchase of vehicles } & 19.50 & 25.50 & 27.70 & 13.70 & 4.50 & 21.10 \\
\hline & & 7.1.1 & Purchase of new cars and vans & [3.00] & 7.00 & 10.10 & 6.20 & [2.00] & 6.60 \\
\hline & & 7.1.2 & Purchase of second hand cars or vans & 15.90 & 16.90 & 16.60 & 7.40 & 2.50 & 13.60 \\
\hline & & 7.1.3 & Purchase of motorcycles and other vehicles & {$[0.70]$} & 1.50 & {$[0.90]$} & [0.10] & - & 0.90 \\
\hline & \multirow[t]{5}{*}{7.2} & \multicolumn{2}{|c|}{ Operation of personal transport } & 24.60 & 38.70 & 40.50 & 22.90 & 9.00 & 31.80 \\
\hline & & 7.2 .1 & Spares and accessories & 1.00 & 2.90 & 3.50 & 1.50 & [0.40] & 2.40 \\
\hline & & 7.2.2 & Petrol, diesel and other motor oils & 17.60 & 25.90 & 26.50 & 14.50 & 5.50 & 21.00 \\
\hline & & 7.2 .3 & Repairs and servicing & 4.10 & 7.40 & 7.70 & 5.40 & 2.50 & 6.20 \\
\hline & & 7.2.4 & Other motoring costs & 1.90 & 2.60 & 2.70 & 1.50 & 0.50 & 2.10 \\
\hline & \multirow[t]{5}{*}{7.3} & \multicolumn{2}{|c|}{ Transport services } & 11.70 & 13.70 & 12.20 & 4.60 & 2.50 & 10.50 \\
\hline & & 7.3.1 & Rail and tube fares & 3.80 & 3.20 & 2.70 & 0.70 & 0.30 & 2.40 \\
\hline & & 7.3.2 & Bus and coach fares & 2.00 & 1.90 & 1.50 & 0.30 & 0.20 & 1.40 \\
\hline & & 7.3.4 & Combined fares & 2.20 & 1.80 & 1.20 & {$[0.00]$} & {$[0.00]$} & 1.20 \\
\hline & & 7.3.5 & Other travel and transport & 3.70 & 6.80 & 6.80 & 3.50 & 2.00 & 5.40 \\
\hline 8 & Com & munica & tion & 13.20 & 14.10 & 13.20 & 8.20 & 5.90 & 12.00 \\
\hline & 8.1 & Postal & services & 0.30 & 0.40 & 0.60 & 0.60 & 0.50 & 0.50 \\
\hline & 8.2 & Teleph & ione and telefax equipment & {$[0.50]$} & 0.50 & 0.80 & {$[0.10]$} & {$[0.10]$} & 0.50 \\
\hline & 8.3 & Teleph & one and telefax services & 12.40 & 13.20 & 11.80 & 7.40 & 5.30 & 11.00 \\
\hline
\end{tabular}

Note: The commodity and service categories are not comparable to those in publications before 2001-02.

The numbering system is sequential, it does not use actual COICOP codes.

Please see page xiii for Symbols and conventions used in this report. 


\section{Table A13}

\section{Detailed household expenditure by age of household reference person, 2008 (cont.)}

based on weighted data and including children's expenditure

\begin{tabular}{|c|c|c|c|c|c|c|c|c|c|}
\hline & & & & $\begin{array}{r}\text { Less } \\
\text { than } \\
30\end{array}$ & $\begin{array}{l}30 \\
\text { to } \\
49\end{array}$ & $\begin{array}{l}50 \\
\text { to } \\
64\end{array}$ & $\begin{array}{l}65 \\
\text { to } \\
74\end{array}$ & $\begin{array}{r}75 \\
\text { or } \\
\text { over }\end{array}$ & $\begin{array}{r}\text { All } \\
\text { house- } \\
\text { holds }\end{array}$ \\
\hline & nmod & ity or s & rvice & & rage we & househo & spenditu & & \\
\hline 9 & Recre & eation & culture & 41.40 & 67.20 & 70.90 & 63.70 & 29.60 & 60.10 \\
\hline & 9.1 & Audio & visual, photographic and information & & & & & & \\
\hline & & proce & sing equipment & 8.40 & 7.40 & 9.10 & 5.90 & 3.10 & 7.20 \\
\hline & & 9.1 .1 & Audio equipment and accessories, CD players & 1.70 & 1.70 & 1.20 & 0.80 & 0.40 & 1.30 \\
\hline & & 9.1 .2 & TV, video and computers & 4.50 & 4.90 & 6.10 & 4.30 & 2.60 & 4.80 \\
\hline & & 9.1 .3 & Photographic, cine and optical equipment & [2.10] & 0.70 & 1.80 & {$[0.80]$} & {$[0.20]$} & 1.10 \\
\hline & 9.2 & Other & major durables for recreation and culture & {$[0.20]$} & 4.10 & 3.40 & [13.60] & {$[0.10]$} & 4.10 \\
\hline & 9.3 & Other & recreational items and equipment, & & & & & & \\
\hline & & garde & s and pets & 8.30 & 13.70 & 12.10 & 7.80 & 4.30 & 10.70 \\
\hline & & 9.3 .1 & Games, toys and hobbies & 2.40 & 2.50 & 1.70 & 1.00 & 0.50 & 1.80 \\
\hline & & 9.3 .2 & Computer software and games & 2.50 & 3.40 & 1.50 & {$[0.20]$} & {$[0.20]$} & 2.00 \\
\hline & & 9.3 .3 & Equipment for sport, camping and & & & & & & \\
\hline & & & open-air recreation & 0.80 & 1.30 & 1.10 & 0.20 & {$[0.10]$} & 0.90 \\
\hline & & 9.3 .4 & Horticultural goods, garden equipment and plants & 0.90 & 2.40 & 3.30 & 3.80 & 2.00 & 2.60 \\
\hline & & 9.3 .5 & Pets and pet food & 1.60 & 4.10 & 4.50 & 2.60 & 1.50 & 3.40 \\
\hline & 9.4 & Recre & tional and cultural services & 14.80 & 21.00 & 20.10 & 14.00 & 6.10 & 17.30 \\
\hline & & 9.4 .1 & Sports admissions, subscriptions, leisure class fees & & & & & & \\
\hline & & & and equipment hire & 3.60 & 6.90 & 5.10 & 3.00 & 0.80 & 4.80 \\
\hline & & 9.4 .2 & Cinema, theatre and museums etc. & 2.10 & 2.40 & 2.10 & 1.50 & 0.70 & 1.90 \\
\hline & & 9.4 .3 & TV, video, satellite rental, cable subscriptions, & & & & & & \\
\hline & & & TV licences and the Internet & 6.20 & 7.10 & 6.50 & 5.00 & 1.50 & 5.80 \\
\hline & & 9.4 .4 & Miscellaneous entertainments & 0.70 & 1.50 & 1.50 & 0.80 & 0.40 & 1.20 \\
\hline & & 9.4 .5 & Development of film, deposit for film development & & & & & & \\
\hline & & & passport photos, holiday and school photos & {$[0.70]$} & 0.30 & 0.30 & 0.20 & [0.10] & 0.30 \\
\hline & & 9.4 .6 & Gambling payments & 1.50 & 2.70 & 4.60 & 3.60 & 2.60 & 3.20 \\
\hline & 9.5 & News & apers, books and stationery & 3.60 & 6.20 & 6.90 & 6.60 & 5.30 & 6.00 \\
\hline & & 9.5 .1 & Books & 1.20 & 1.70 & 1.50 & 1.00 & 0.60 & 1.40 \\
\hline & & 9.5 .2 & Diaries, address books, cards etc. & 1.30 & 2.20 & 2.20 & 1.80 & 1.10 & 1.90 \\
\hline & & 9.5 .3 & Newspapers & 0.50 & 1.20 & 2.30 & 2.90 & 2.90 & 1.90 \\
\hline & & 9.5 .4 & Magazines and periodicals & 0.60 & 1.00 & 1.00 & 0.80 & 0.70 & 0.90 \\
\hline & 9.6 & Packa & e holidays & 6.30 & 14.90 & 19.30 & 15.80 & 10.80 & 14.70 \\
\hline & & 9.6 .1 & Package holidays - UK & [0.90] & 0.60 & 0.70 & 1.80 & 2.80 & 1.10 \\
\hline & & 9.6 .2 & Package holidays - abroad & 5.40 & 14.30 & 18.60 & 14.00 & 8.00 & 13.60 \\
\hline 10 & Educ & ation & & 3.00 & 8.90 & 8.80 & {$[1.30]$} & {$[0.40]$} & 6.20 \\
\hline & 10.1 & Educa & ion fees & 2.80 & 8.30 & 8.60 & {$[1.20]$} & {$[0.40]$} & 5.90 \\
\hline & 10.2 & $\begin{array}{l}\text { Paym } \\
\text { expen }\end{array}$ & $\begin{array}{l}\text { nts for school trips, other ad-hoc } \\
\text { diture }\end{array}$ & {$[0.10]$} & 0.60 & 0.20 & {$[0.00]$} & - & 0.30 \\
\hline 11 & Rest & aurants & \& hotels & 38.30 & 46.40 & 44.00 & 25.10 & 12.60 & 37.70 \\
\hline & 11.1 & Cater & ig services & 34.60 & 38.30 & 35.30 & 19.50 & 10.00 & 31.00 \\
\hline & & 11.1.1 & Restaurant and café meals & 9.40 & 15.10 & 16.00 & 11.60 & 7.00 & 13.20 \\
\hline & & 11.1.2 & Alcoholic drinks (away from home) & 7.60 & 8.40 & 9.40 & 4.60 & 1.70 & 7.20 \\
\hline & & 11.1.3 & Take away meals eaten at home & 5.90 & 5.20 & 3.60 & 1.80 & 1.00 & 3.90 \\
\hline & & 11.1.4 & Other take-away and snack food & 5.10 & 6.30 & 4.40 & 0.90 & 0.30 & 4.20 \\
\hline & & 11.1.5 & Contract catering (food) and canteens & 6.60 & 3.40 & 2.00 & 0.40 & {$[0.00]$} & 2.50 \\
\hline & 11.2 & Accon & modation services & 3.70 & 8.10 & 8.70 & 5.70 & 2.50 & 6.80 \\
\hline & & 11.2.1 & Holiday in the UK & 0.80 & 2.80 & 3.50 & 3.70 & 1.70 & 2.80 \\
\hline & & 11.2 .2 & Holiday abroad & 2.80 & 5.20 & 5.20 & 2.00 & {$[0.80]$} & 4.00 \\
\hline & & 11.2 .3 & Room hire & {$[0.10]$} & {$[0.00]$} & {$[0.00]$} & - & {$[0.00]$} & {$[0.00]$} \\
\hline
\end{tabular}

Note: The commodity and service categories are not comparable to those in publications before 2001-02.

The numbering system is sequential, it does not use actual COICOP codes.

Please see page xiii for Symbols and conventions used in this report. 


\section{Table A13}

Detailed household expenditure by age of household reference person, 2008 (cont.) based on weighted data and including children's expenditure

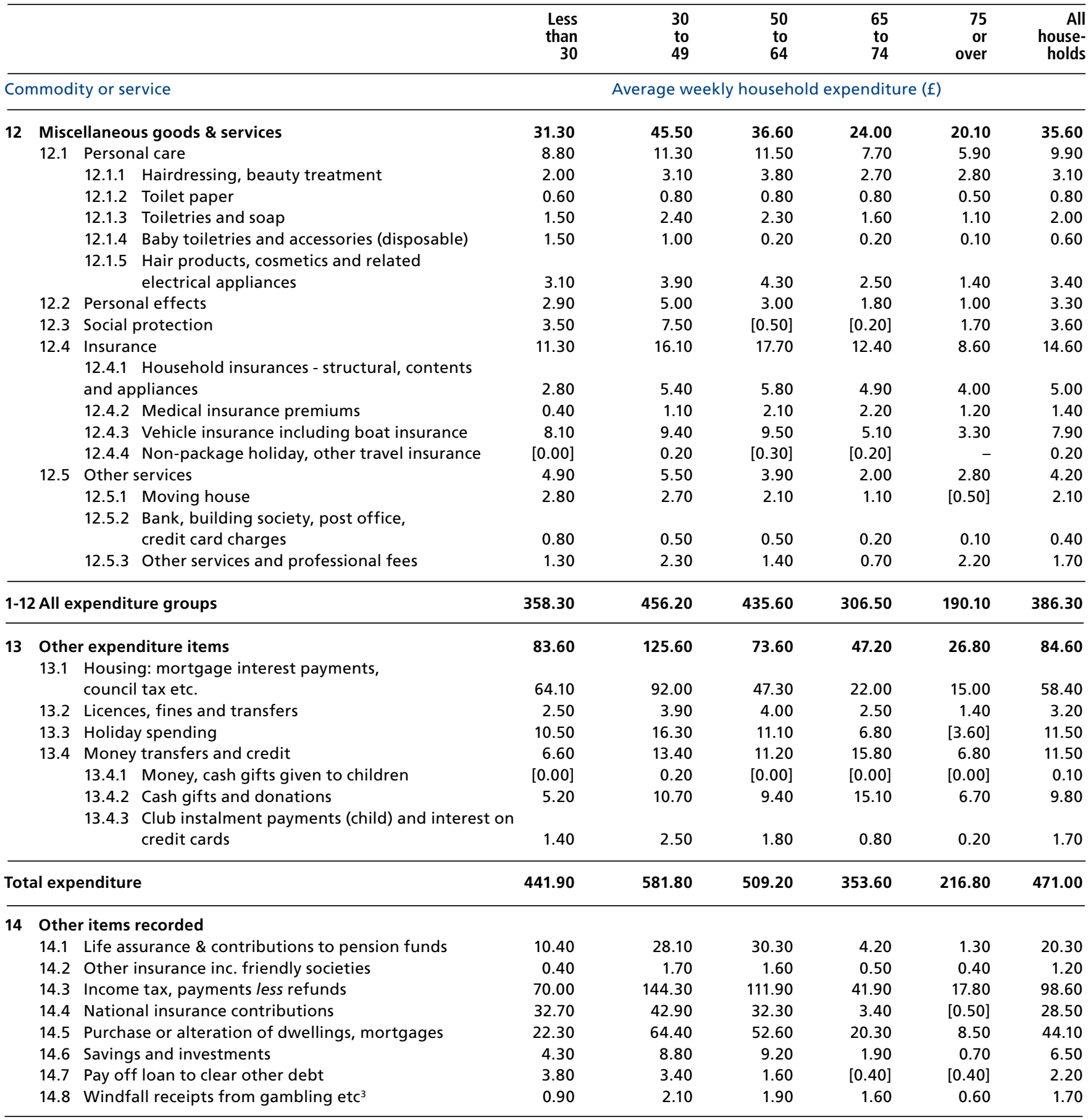

Note: The commodity and service categories are not comparable to those in publications before 2001-02.

The numbering system is sequential, it does not use actual COICOP codes.

Please see page xiii for Symbols and conventions used in this report.

3 Expressed as an income figure as opposed to an expenditure figure. 


\section{Table A14}

Household expenditure by gross income quintile group where the household reference person is aged under 30, 2006-2008

based on weighted data and including children's expenditure

\begin{tabular}{|c|c|c|c|c|c|c|c|}
\hline & & $\begin{array}{r}\text { Lowest } \\
\text { twenty } \\
\text { per cent }\end{array}$ & $\begin{array}{l}\text { Second } \\
\text { quintile } \\
\text { group }\end{array}$ & $\begin{array}{r}\text { Third } \\
\text { quintile } \\
\text { group }\end{array}$ & $\begin{array}{r}\text { Fourth } \\
\text { quintile } \\
\text { group }\end{array}$ & $\begin{array}{c}\text { Highest } \\
\text { twenty } \\
\text { per cent }\end{array}$ & $\begin{array}{r}\text { All } \\
\text { house- } \\
\text { holds }\end{array}$ \\
\hline \multicolumn{2}{|c|}{ Lower boundary of group ( $\mathrm{f}$ per week) ${ }^{1}$} & & 223 & 408 & 664 & 1026 & \\
\hline \multirow{5}{*}{\multicolumn{2}{|c|}{$\begin{array}{l}\text { Average weighted number of households (thousands) } \\
\text { Total number of households in sample (over } 3 \text { years) } \\
\text { Total number of persons in sample (over } 3 \text { years) } \\
\text { Total number of adults in sample (over } 3 \text { years) } \\
\text { Weighted average number of persons per household }\end{array}$}} & 530 & 420 & 660 & 620 & 370 & 2,600 \\
\hline & & 360 & 300 & 410 & 370 & 210 & 1,650 \\
\hline & & 790 & 760 & 1,020 & 930 & 540 & 4,040 \\
\hline & & 460 & 450 & 750 & 780 & 480 & 2,920 \\
\hline & & 2.1 & 2.4 & 2.5 & 2.5 & 2.5 & 2.4 \\
\hline \multicolumn{2}{|c|}{ Commodity or service } & \multicolumn{6}{|c|}{ Average weekly household expenditure (f) } \\
\hline 1 & Food \& non-alcoholic drinks & 27.10 & 30.20 & 37.60 & 42.90 & 49.40 & 37.20 \\
\hline 2 & Alcoholic drinks, tobacco \& narcotics & 8.80 & 10.10 & 10.40 & 12.70 & 11.50 & 10.70 \\
\hline 3 & Clothing \& footwear & 11.20 & 17.30 & 21.30 & 32.50 & 39.10 & 23.70 \\
\hline 4 & Housing(net) ${ }^{2}$, fuel \& power & 51.60 & 74.60 & 87.70 & 83.70 & 92.60 & 77.90 \\
\hline 5 & Household goods \& services & 14.10 & 15.50 & 22.10 & 34.70 & 43.40 & 25.40 \\
\hline 6 & Health & 0.80 & 1.40 & 2.90 & 4.10 & 3.90 & 2.70 \\
\hline 7 & Transport & 15.00 & 30.90 & 47.20 & 73.10 & 123.30 & 55.00 \\
\hline 8 & Communication & 7.30 & 10.90 & 14.70 & 17.30 & 19.40 & 13.90 \\
\hline 9 & Recreation \& culture & 18.70 & 27.80 & 44.00 & 62.40 & 71.00 & 44.40 \\
\hline 10 & Education & [7.30] & 6.20 & 8.10 & 8.40 & [4.50] & 7.10 \\
\hline 11 & Restaurants \& hotels & 15.90 & 23.00 & 36.50 & 53.30 & 72.60 & 39.20 \\
\hline 12 & Miscellaneous goods \& services & 10.30 & 20.50 & 34.50 & 45.10 & 54.20 & 32.60 \\
\hline $1-12$ & All expenditure groups & 188.10 & 268.40 & 367.10 & 470.30 & 584.90 & 369.70 \\
\hline 13 & Other expenditure items & 8.00 & 37.20 & 69.70 & 107.80 & 166.90 & 74.80 \\
\hline \multicolumn{2}{|c|}{ Total expenditure } & 196.10 & 305.60 & 436.80 & $\mathbf{5 7 8 . 1 0}$ & 751.70 & 444.50 \\
\hline \multicolumn{2}{|c|}{$\begin{array}{l}\text { Average weekly expenditure per person (f) } \\
\text { Total expenditure }\end{array}$} & 91.80 & 128.50 & 175.50 & 235.10 & 295.80 & 185.30 \\
\hline
\end{tabular}

Note: The commodity and service categories are not comparable to those in publications before 2001-02.

Please see page xiii for symbols and conventions used in this report.

This table is based on a three year average.

1 Lower boundary of 2008 gross income quintile groups ( $f$ per week ).

2 Excluding mortgage interest payments, council tax and Northern Ireland rates. 


\section{Table A15}

\section{Household expenditure by gross income quintile group where the} household reference person is aged under 30 to 49, 2006-2008

based on weighted data and including children's expenditure

\begin{tabular}{|c|c|c|c|c|c|c|c|}
\hline & & $\begin{array}{l}\text { Lowest } \\
\text { twenty } \\
\text { per cent }\end{array}$ & $\begin{array}{l}\text { Second } \\
\text { quintile } \\
\text { group }\end{array}$ & $\begin{array}{l}\text { Third } \\
\text { quintile } \\
\text { group }\end{array}$ & $\begin{array}{r}\text { Fourth } \\
\text { quintile } \\
\text { group }\end{array}$ & $\begin{array}{l}\text { Highest } \\
\text { twenty } \\
\text { per cent }\end{array}$ & $\begin{array}{r}\text { All } \\
\text { house- } \\
\text { holds }\end{array}$ \\
\hline \multicolumn{2}{|c|}{ Lower boundary of group ( $\mathrm{f}$ per week) ${ }^{1}$} & & 223 & 408 & 664 & 1026 & \\
\hline \multicolumn{2}{|r|}{ Average weighted number of households (thousands) } & 1,030 & 1,260 & 1,990 & 2,590 & 2,910 & 9,770 \\
\hline \multicolumn{2}{|r|}{ Total number of households in sample (over 3 years) } & 750 & 960 & 1,510 & 1,890 & 2,020 & 7,130 \\
\hline \multicolumn{2}{|r|}{ Total number of persons in sample (over 3 years) } & 1,600 & 2,680 & 4,530 & 6,060 & 6,760 & 21,630 \\
\hline \multicolumn{2}{|c|}{ Total number of adults in sample (over 3 years) } & 940 & 1,450 & 2,700 & 3,840 & 4,440 & 13,370 \\
\hline \multicolumn{2}{|r|}{ Weighted average number of persons per household } & 2.1 & 2.7 & 2.9 & 3.1 & 3.2 & 2.9 \\
\hline \multicolumn{2}{|c|}{ Commodity or service } & \multicolumn{6}{|c|}{ Average weekly household expenditure (f) } \\
\hline 1 & Food \& non-alcoholic drinks & 32.00 & 41.40 & 49.30 & 57.80 & 68.30 & 54.30 \\
\hline 2 & Alcoholic drinks, tobacco \& narcotics & 8.30 & 10.50 & 11.60 & 13.00 & 15.40 & 12.60 \\
\hline 3 & Clothing \& footwear & 10.70 & 20.50 & 21.20 & 29.00 & 43.60 & 28.70 \\
\hline 4 & Housing(net) ${ }^{2}$, fuel \& power & 44.00 & 55.20 & 54.10 & 54.10 & 63.00 & 55.80 \\
\hline 5 & Household goods \& services & 12.90 & 20.60 & 24.80 & 36.80 & 56.80 & 35.70 \\
\hline 6 & Health & 1.70 & 2.20 & 3.50 & 4.10 & 7.90 & 4.60 \\
\hline 7 & Transport & 20.30 & 40.70 & 55.80 & 76.00 & 130.20 & 77.60 \\
\hline 8 & Communication & 8.50 & 11.40 & 13.80 & 14.40 & 17.30 & 14.10 \\
\hline 9 & Recreation \& culture & 21.80 & 35.70 & 49.70 & 71.60 & 104.20 & 67.00 \\
\hline 10 & Education & 2.80 & 3.10 & 2.60 & 4.60 & 23.70 & 9.50 \\
\hline 11 & Restaurants \& hotels & 14.90 & 24.80 & 33.20 & 47.40 & 76.30 & 46.80 \\
\hline 12 & Miscellaneous goods \& services & 15.40 & 23.90 & 33.50 & 45.80 & 68.40 & 44.00 \\
\hline \multicolumn{2}{|c|}{ 1-12 All expenditure groups } & 193.10 & 289.80 & 353.00 & 454.60 & 675.10 & 450.80 \\
\hline 13 & Other expenditure items & 23.20 & 54.00 & 79.20 & 114.30 & 194.20 & 113.50 \\
\hline \multicolumn{8}{|c|}{ Average weekly expenditure per person (f) } \\
\hline Tot & expenditure & 105.10 & 127.90 & 149.60 & 182.00 & 267.70 & 191.60 \\
\hline
\end{tabular}

Note: The commodity and service categories are not comparable to those in publications before 2001-02.

Please see page xiii for symbols and conventions used in this report.

This table is based on a three year average.

1 Lower boundary of 2008 gross income quintile groups ( $f$ per week).

2 Excluding mortgage interest payments, council tax and Northern Ireland rates. 


\section{Table A16}

\section{Household expenditure by gross income quintile group where the household reference person is aged 50 to 64, 2006-2008}

based on weighted data and including children's expenditure

\begin{tabular}{|c|c|c|c|c|c|c|c|}
\hline & & $\begin{array}{r}\text { Lowest } \\
\text { twenty } \\
\text { per cent }\end{array}$ & $\begin{array}{l}\text { Second } \\
\text { quintile } \\
\text { group }\end{array}$ & $\begin{array}{r}\text { Third } \\
\text { quintile } \\
\text { group }\end{array}$ & $\begin{array}{r}\text { Fourth } \\
\text { quintile } \\
\text { group }\end{array}$ & $\begin{array}{c}\text { Highest } \\
\text { twenty } \\
\text { per cent }\end{array}$ & $\begin{array}{r}\text { All } \\
\text { house- } \\
\text { holds }\end{array}$ \\
\hline \multicolumn{2}{|c|}{ Lower boundary of group ( $\mathrm{f}$ per week) ${ }^{1}$} & & 223 & 408 & 664 & 1026 & \\
\hline \multicolumn{2}{|c|}{ Weighted number of households (thousands) } & 1,060 & 1,120 & 1,390 & 1,390 & 1,590 & 6,550 \\
\hline \multicolumn{2}{|r|}{ Total number of households in sample (over 3 years) } & 820 & 870 & 1,070 & 1,050 & 1,130 & 4,950 \\
\hline \multicolumn{2}{|r|}{ Total number of persons in sample (over 3 years) } & 1,150 & 1,550 & 2,270 & 2,560 & 3,270 & 10,790 \\
\hline \multirow{2}{*}{\multicolumn{2}{|c|}{$\begin{array}{l}\text { Total number of adults in sample (over } 3 \text { years) } \\
\text { Weighted average number of persons per household }\end{array}$}} & 1,070 & 1,420 & 2,070 & 2,320 & 2,900 & 9,790 \\
\hline & & 1.4 & 1.8 & 2.1 & 2.4 & 3.0 & 2.2 \\
\hline \multicolumn{2}{|c|}{ Commodity or service } & \multicolumn{6}{|c|}{ Average weekly household expenditure (f) } \\
\hline 1 & Food \& non-alcoholic drinks & 30.00 & 40.40 & 50.50 & 59.20 & 75.70 & 53.40 \\
\hline 2 & Alcoholic drinks, tobacco \& narcotics & 7.80 & 10.60 & 12.70 & 15.20 & 16.40 & 13.00 \\
\hline 3 & Clothing \& footwear & 8.10 & 13.40 & 19.00 & 24.00 & 45.00 & 23.60 \\
\hline 4 & Housing(net) $)^{2}$, fuel \& power & 34.60 & 44.10 & 45.40 & 47.10 & 57.60 & 46.70 \\
\hline 5 & Household goods \& services & 13.30 & 24.20 & 28.30 & 36.20 & 55.00 & 33.30 \\
\hline 6 & Health & 2.50 & 4.00 & 6.40 & 7.40 & 17.50 & 8.20 \\
\hline 7 & Transport & 22.80 & 39.90 & 60.50 & 78.10 & 143.10 & 74.60 \\
\hline 8 & Communication & 6.90 & 9.50 & 11.60 & 13.80 & 17.70 & 12.40 \\
\hline 9 & Recreation \& culture & 24.90 & 42.80 & 58.30 & 75.10 & 118.60 & 68.40 \\
\hline 10 & Education & {$[0.60]$} & 1.00 & 3.20 & 9.70 & 20.20 & 8.00 \\
\hline 11 & Restaurants \& hotels & 12.00 & 22.40 & 32.80 & 49.70 & 79.30 & 42.50 \\
\hline 12 & Miscellaneous goods \& services & 13.20 & 23.10 & 29.50 & 41.60 & 66.00 & 37.10 \\
\hline $1-12$ & All expenditure groups & 176.80 & 275.50 & 358.10 & 457.10 & 712.10 & 421.40 \\
\hline 13 & Other expenditure items & 20.50 & 49.30 & 59.10 & 82.50 & 144.70 & 76.90 \\
\hline \multicolumn{2}{|c|}{ Total expenditure } & 197.20 & 324.80 & 417.20 & 539.60 & 856.80 & 498.30 \\
\hline \multicolumn{8}{|c|}{ Average weekly expenditure per person (f) } \\
\hline
\end{tabular}

Note: The commodity and service categories are not comparable to those in publications before 2001-02. Please see page xiii for symbols and conventions used in this report.

This table is based on a three year average.

1 Lower boundary of 2008 gross income quintile groups ( $f$ per week ).

2 Excluding mortgage interest payments, council tax and Northern Ireland rates. 


\section{Table A17}

\section{Household expenditure by gross income quintile group where the household reference person is aged 65 to 74, 2006-2008}

based on weighted data and including children's expenditure

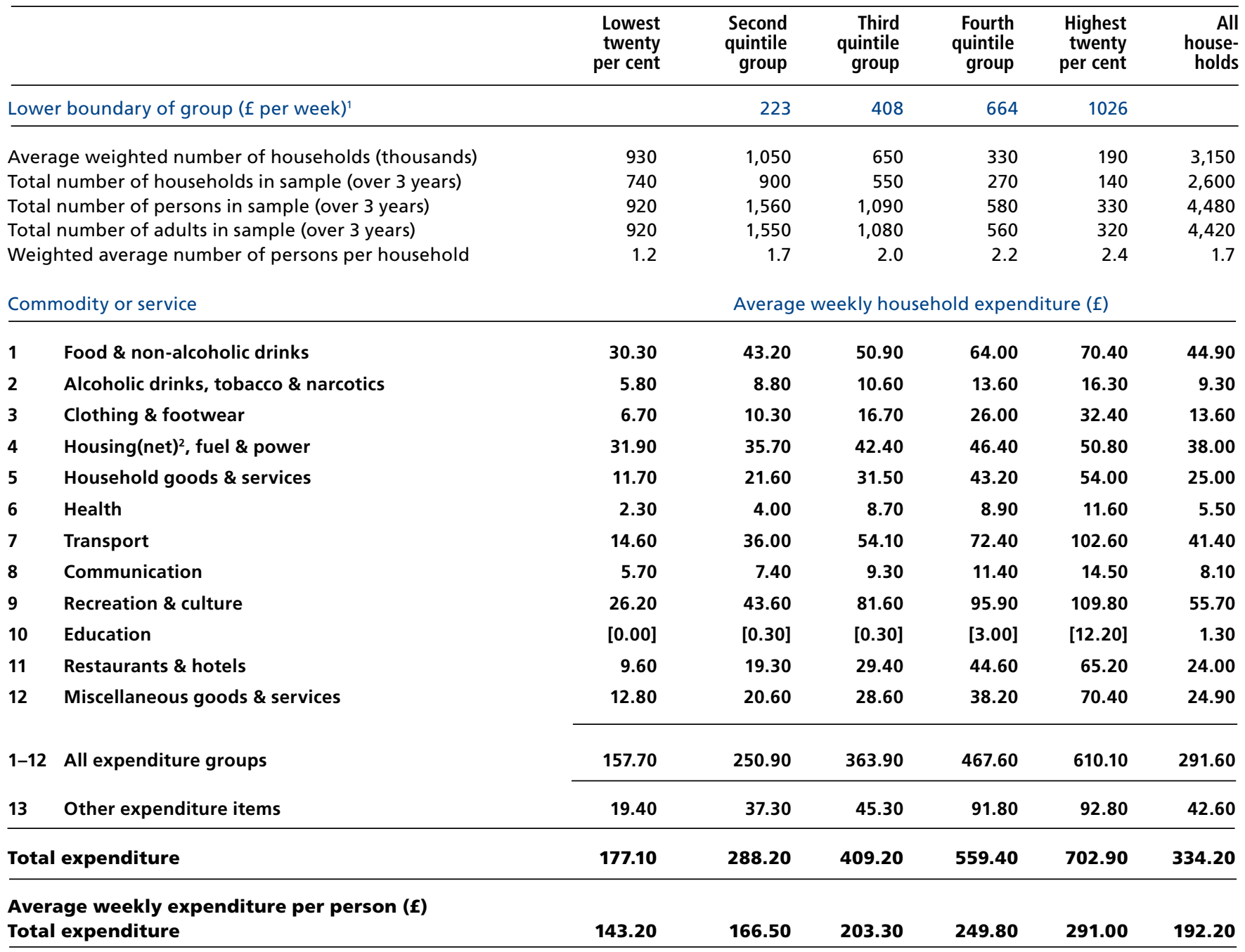

Note: The commodity and service categories are not comparable to those in publications before 2001-02.

Please see page xiii for symbols and conventions used in this report.

This table is based on a three year average.

1 Lower boundary of 2008 gross income quintile groups ( $f$ per week ).

2 Excluding mortgage interest payments, council tax and Northern Ireland rates. 


\section{Table A18}

\section{Household expenditure by gross income quintile group where the household reference person is aged 75 or over, 2006-2008}

based on weighted data and including children's expenditure

\begin{tabular}{|c|c|c|c|c|c|c|c|}
\hline & & $\begin{array}{l}\text { Lowest } \\
\text { twenty } \\
\text { per cent }\end{array}$ & $\begin{array}{l}\text { Second } \\
\text { quintile } \\
\text { group }\end{array}$ & $\begin{array}{r}\text { Third } \\
\text { quintile } \\
\text { group }\end{array}$ & $\begin{array}{r}\text { Fourth } \\
\text { quintile } \\
\text { group }\end{array}$ & $\begin{array}{l}\text { Highest } \\
\text { twenty } \\
\text { per cent }\end{array}$ & $\begin{array}{r}\text { All } \\
\text { house- } \\
\text { holds }\end{array}$ \\
\hline \multicolumn{2}{|c|}{ Lower boundary of group ( $\mathrm{f}$ per week) ${ }^{1}$} & & 223 & 408 & 664 & 1026 & \\
\hline \multicolumn{2}{|c|}{ Average weighted number of households (thousands) } & 1,570 & 1,220 & 400 & 170 & 50 & 3,410 \\
\hline \multicolumn{2}{|r|}{ Total number of households in sample (over 3 years) } & 990 & 860 & 300 & 120 & 40 & 2,310 \\
\hline \multicolumn{2}{|r|}{ Total number of persons in sample (over 3 years) } & 1,130 & 1,370 & 540 & 260 & 100 & 3,390 \\
\hline \multicolumn{2}{|r|}{ Total number of adults in sample (over 3 years) } & 1,120 & 1,360 & 540 & 250 & 100 & 3,370 \\
\hline \multicolumn{2}{|r|}{ Weighted average number of persons per household } & 1.1 & 1.5 & 1.8 & 2.1 & 2.7 & 1.4 \\
\hline \multicolumn{2}{|c|}{ Commodity or service } & \multicolumn{6}{|c|}{ Average weekly household expenditure $(f)$} \\
\hline 1 & Food \& non-alcoholic drinks & 24.80 & 35.60 & 45.80 & 57.80 & 68.50 & 33.40 \\
\hline 2 & Alcoholic drinks, tobacco \& narcotics & 2.80 & 5.00 & 7.70 & 8.40 & 12.50 & 4.60 \\
\hline 4 & Housing (net) ${ }^{2}$, fuel \& power & 30.10 & 36.70 & 42.60 & 45.20 & 63.10 & 35.10 \\
\hline 5 & Household goods \& services & 12.70 & 16.60 & 26.60 & 36.00 & 50.50 & 17.50 \\
\hline 6 & Health & 2.80 & 6.20 & 10.00 & 11.50 & 8.50 & 5.40 \\
\hline 7 & Transport & 6.50 & 18.50 & 32.90 & 44.50 & 97.20 & 17.20 \\
\hline 8 & Communication & 4.70 & 5.80 & 8.70 & 10.30 & 11.20 & 5.90 \\
\hline 9 & Recreation \& culture & 13.90 & 27.10 & 52.60 & 65.60 & 125.70 & 27.50 \\
\hline 10 & Education & {$[0.00]$} & {$[0.20]$} & {$[2.30]$} & {$[5.10]$} & {$[2.20]$} & {$[0.60]$} \\
\hline 11 & Restaurants \& hotels & 6.60 & 11.40 & 20.50 & 47.20 & 46.10 & 12.50 \\
\hline 12 & Miscellaneous goods \& services & 11.50 & 18.80 & 35.00 & 56.20 & 94.40 & 20.30 \\
\hline $1-12$ & All expenditure groups & 121.20 & 188.90 & 295.60 & 406.70 & 611.50 & 187.70 \\
\hline 13 & Other expenditure items & 14.90 & 25.70 & 43.00 & 74.10 & 105.90 & 26.30 \\
\hline \multicolumn{2}{|r|}{$\begin{array}{l}\text { age weekly expenditure per person (f) } \\
\text { lexpenditure }\end{array}$} & 122.00 & 140.70 & 189.50 & 226.60 & 265.30 & 151.10 \\
\hline
\end{tabular}

Note: The commodity and service categories are not comparable to those in publications before 2001-02.

Please see page xiii for symbols and conventions used in this report.

This table is based on a three year average.

1 Lower boundary of 2008 gross income quintile groups ( $f$ per week ).

2 Excluding mortgage interest payments, council tax and Northern Ireland rates. 


\section{Table A19}

\section{Household expenditure by economic activity status of the}

\section{household reference person, 2008}

based on weighted data and including children's expenditure

\begin{tabular}{|c|c|c|c|c|c|c|}
\hline & & \multicolumn{3}{|c|}{ Employees } & \multirow{2}{*}{$\begin{array}{r}\text { Self- } \\
\text { employed }\end{array}$} & \multirow{2}{*}{$\begin{array}{r}\text { All in } \\
\text { employment }\end{array}$} \\
\hline & & Full-time & Part-time & All & & \\
\hline \multicolumn{2}{|r|}{ Weighted number of households (thousands) } & 11,400 & 2,150 & 13,550 & 1,960 & 15,570 \\
\hline \multicolumn{2}{|c|}{ Total number of households in sample } & 2,510 & 500 & 3,010 & 470 & 3,490 \\
\hline \multicolumn{2}{|c|}{ Total number of persons in sample } & 6,780 & 1,260 & 8,040 & 1,360 & 9,430 \\
\hline \multirow{2}{*}{\multicolumn{2}{|c|}{$\begin{array}{l}\text { Total number of adults in sample } \\
\text { Weighted average number of persons per household }\end{array}$}} & 5,000 & 880 & 5,880 & 990 & 6,880 \\
\hline & & 2.7 & 2.5 & 2.7 & 2.8 & 2.7 \\
\hline \multicolumn{2}{|c|}{ Commodity or service } & \multicolumn{4}{|c|}{ Average weekly household expenditure (f) } & \\
\hline 1 & Food \& non-alcoholic drinks & 56.70 & 50.60 & 55.80 & 64.10 & 56.80 \\
\hline 2 & Alcoholic drinks, tobacco \& narcotics & 12.70 & 10.50 & 12.40 & 12.40 & 12.40 \\
\hline 3 & Clothing \& footwear & 28.20 & 23.80 & 27.50 & 27.60 & 27.50 \\
\hline 4 & Housing (net) ${ }^{2}$, fuel \& power & 60.90 & 61.40 & 61.00 & 63.30 & 61.20 \\
\hline 5 & Household goods \& services & 36.10 & 28.50 & 34.90 & 43.70 & 36.20 \\
\hline 6 & Health & 6.20 & 4.60 & 6.00 & 4.90 & 5.90 \\
\hline 7 & Transport & 87.90 & 53.80 & 82.50 & 96.30 & 84.20 \\
\hline 8 & Communication & 14.30 & 12.70 & 14.10 & 17.80 & 14.50 \\
\hline 9 & Recreation \& culture & 72.30 & 60.20 & 70.40 & 76.80 & 71.10 \\
\hline 10 & Education & 9.40 & 6.90 & 9.00 & 12.30 & 9.40 \\
\hline 11 & Restaurants \& hotels & 49.70 & 34.80 & 47.40 & 57.50 & 48.60 \\
\hline 12 & Miscellaneous goods \& services & 46.90 & 33.20 & 44.70 & 45.20 & 44.80 \\
\hline 1-12 & All expenditure groups & 481.50 & 381.00 & 465.60 & 521.80 & 472.70 \\
\hline 13 & Other expenditure items & 126.40 & 63.60 & 116.40 & 126.10 & 117.50 \\
\hline \multicolumn{2}{|c|}{ Total expenditure } & 608.00 & 444.60 & 582.00 & 647.90 & 590.20 \\
\hline \multicolumn{2}{|r|}{$\begin{array}{l}\text { Average weekly expenditure per person (f) } \\
\text { Total expenditure }\end{array}$} & 226.70 & 175,10 & 21890 & 27,50 & 23000 \\
\hline
\end{tabular}

Note: The commodity and service categories are not comparable to those in publications before 2001-02. Please see page xiii for symbols and conventions used in this report.

1 Includes households where household reference person was on a government supported training scheme.

2 Excluding mortgage interest payments, council tax and Northern Ireland rates. 


\section{Table A19}

Household expenditure by economic activity status of the household reference person, 2008 (cont.)

based on weighted data and including children's expenditure

\begin{tabular}{|c|c|c|c|c|c|c|}
\hline & \multirow{2}{*}{$\begin{array}{l}\text { Unem- } \\
\text { ployed }\end{array}$} & \multirow{2}{*}{$\begin{array}{r}\text { All } \\
\text { economi- } \\
\text { cally active }^{1}\end{array}$} & \multicolumn{3}{|c|}{ Economically inactive } & \multirow{2}{*}{$\begin{array}{r}\text { All } \\
\text { house- } \\
\text { holds }\end{array}$} \\
\hline & & & Retired & Other & All & \\
\hline Weighted number of households (thousands) & 500 & 16,070 & 6,660 & 2,960 & 9,620 & 25,690 \\
\hline Total number of households in sample & 100 & 3,590 & 1,560 & 700 & 2,250 & 5,850 \\
\hline Total number of persons in sample & 240 & 9,670 & 2,460 & 1,700 & 4,160 & 13,830 \\
\hline Total number of adults in sample & 150 & 7,030 & 2,440 & 1,170 & 3,610 & 10,640 \\
\hline Weighted average number of persons per household & 2.4 & 2.7 & 1.5 & 2.5 & 1.8 & 2.4 \\
\hline
\end{tabular}

Commodity or service

Average weekly household expenditure (f)

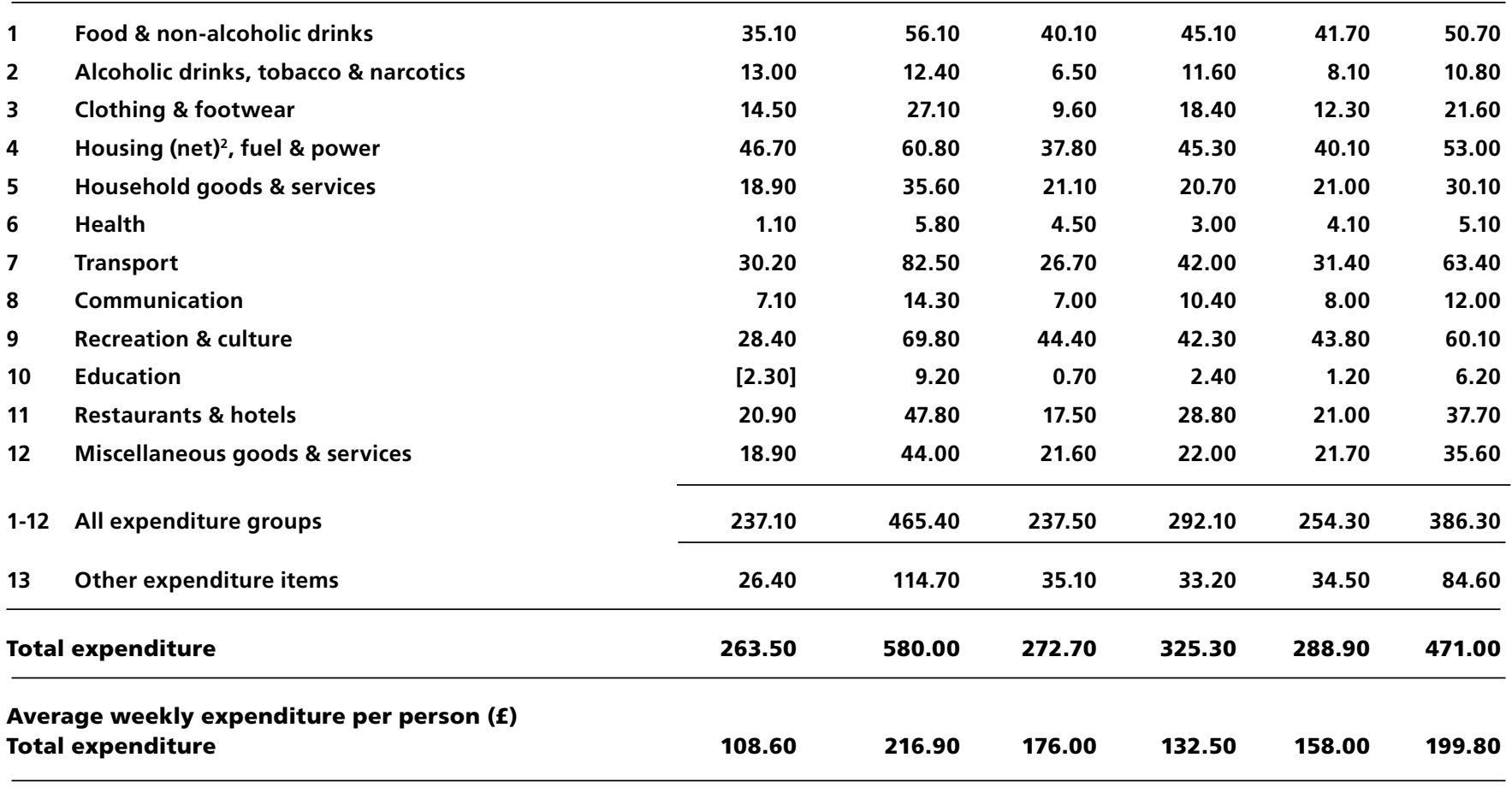

Note: The commodity and service categories are not comparable to those in publications before $2001-02$. Please see page xiii for symbols and conventions used in this report.

1 Includes households where household reference person was on a government supported training scheme.

2 Excluding mortgage interest payments, council tax and Northern Ireland rates. 


\section{Table A20}

Household expenditure by gross income quintile group: the household reference person is a full-time employee, 2008

based on weighted data and including children's expenditure

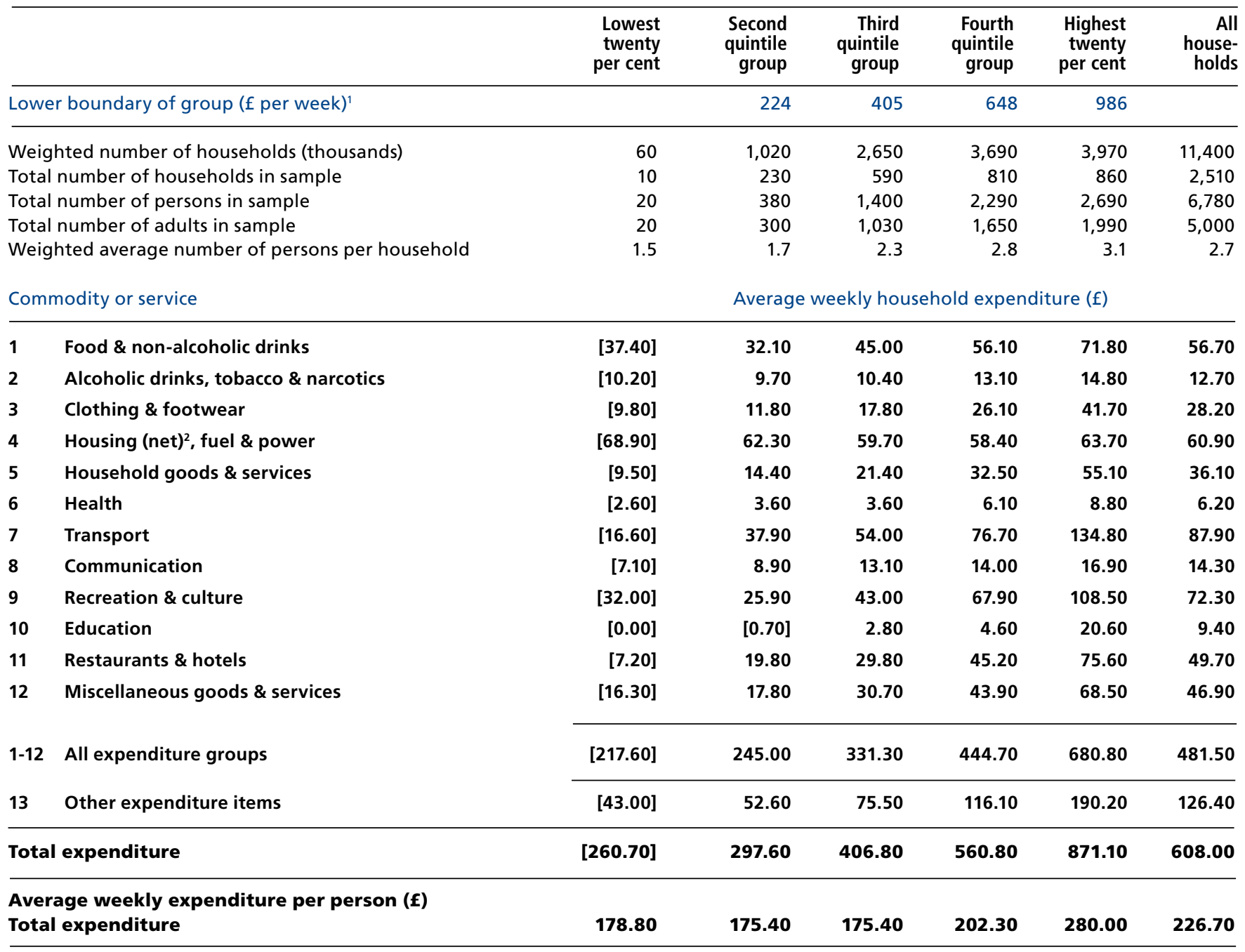

Note: The commodity and service categories are not comparable to those in publications before 2001-02.

Please see page xiii for symbols and conventions used in this report.

1 Lower boundary of 2008 gross income quintile groups ( $f$ per week ).

2 Excluding mortgage interest payments, council tax and Northern Ireland rates. 


\section{Table A21}

Household expenditure by gross income quintile group: the household reference person is self-employed, 2006-2008

based on weighted data and including children's expenditure

\begin{tabular}{|c|c|c|c|c|c|c|c|}
\hline & & $\begin{array}{r}\text { Lowest } \\
\text { twenty } \\
\text { per cent }\end{array}$ & $\begin{array}{l}\text { Second } \\
\text { quintile } \\
\text { group }\end{array}$ & $\begin{array}{l}\text { Third } \\
\text { quintile } \\
\text { group }\end{array}$ & $\begin{array}{l}\text { Fourth } \\
\text { quintile } \\
\text { group }\end{array}$ & $\begin{array}{l}\text { Highest } \\
\text { twenty } \\
\text { per cent }\end{array}$ & $\begin{array}{r}\text { All } \\
\text { house- } \\
\text { holds }\end{array}$ \\
\hline \multicolumn{2}{|c|}{ Lower boundary of group ( $\mathrm{f}$ per week) ${ }^{1}$} & & 223 & 408 & 664 & 1026 & \\
\hline \multicolumn{2}{|r|}{ Average weighted number of households (thousands) } & 180 & 320 & 450 & 480 & 600 & 2,040 \\
\hline \multicolumn{2}{|r|}{ Total number of households in sample (over 3 years) } & 130 & 250 & 360 & 360 & 440 & 1,530 \\
\hline \multicolumn{2}{|r|}{ Total number of persons in sample (over 3 years) } & 250 & 630 & 1,060 & 1,070 & 1,390 & 4,400 \\
\hline \multicolumn{2}{|c|}{ Total number of adults in sample (over 3 years) } & 190 & 430 & 740 & 770 & 1,020 & 3,160 \\
\hline \multicolumn{2}{|r|}{ Weighted average number of persons per household } & 1.9 & 2.5 & 2.9 & 2.9 & 3.1 & 2.8 \\
\hline \multicolumn{2}{|c|}{ Commodity or service } & \multicolumn{6}{|c|}{ Average weekly household expenditure (f) } \\
\hline 1 & Food \& non-alcoholic drinks & 36.10 & 46.20 & 59.00 & 64.30 & 73.00 & 60.40 \\
\hline 2 & Alcoholic drinks, tobacco \& narcotics & 6.10 & 11.20 & 14.40 & 14.80 & 15.10 & 13.50 \\
\hline 3 & Clothing \& footwear & 10.50 & 21.60 & 24.60 & 27.20 & 49.70 & 31.00 \\
\hline 4 & Housing(net)², fuel \& power & 53.40 & 59.50 & 55.10 & 62.80 & 60.70 & 59.00 \\
\hline 5 & Household goods \& services & 20.60 & 29.10 & 30.00 & 40.40 & 62.90 & 41.10 \\
\hline 6 & Health & 2.40 & 3.80 & 6.20 & 4.20 & 9.70 & 6.10 \\
\hline 7 & Transport & 47.60 & 58.30 & 63.40 & 87.50 & 136.40 & 88.40 \\
\hline 8 & Communication & 10.40 & 13.30 & 15.50 & 18.20 & 20.50 & 16.80 \\
\hline 9 & Recreation \& culture & 27.90 & 47.60 & 58.40 & 77.40 & 123.30 & 78.20 \\
\hline 10 & Education & {$[5.50]$} & {$[2.30]$} & 4.00 & 10.80 & 30.30 & 13.20 \\
\hline 11 & Restaurants \& hotels & 23.70 & 32.20 & 40.10 & 57.50 & 80.60 & 53.70 \\
\hline 12 & Miscellaneous goods \& services & 30.20 & 30.50 & 41.30 & 45.30 & 68.90 & 47.90 \\
\hline $1-12$ & All expenditure groups & 274.40 & 355.50 & 412.10 & 510.50 & 731.30 & 509.30 \\
\hline 13 & Other expenditure items & 75.60 & 81.70 & 94.50 & 112.50 & 179.80 & 120.40 \\
\hline \multicolumn{2}{|c|}{ Total expenditure } & 350.00 & 437.10 & 506.60 & 623.00 & 911.10 & 629.70 \\
\hline \multicolumn{8}{|c|}{ Average weekly expenditure per person (f) } \\
\hline \multicolumn{2}{|c|}{ Total expenditure } & 181.80 & 177.60 & 176.90 & 211.90 & 289.70 & 223.00 \\
\hline
\end{tabular}

Note: The commodity and service categories are not comparable to those in publications before 2001-02.

Please see page xiii for symbols and conventions used in this report.

This table is based on a three year average.

1 Lower boundary of 2008 gross income quintile groups ( $f$ per week ).

2 Excluding mortgage interest payments, council tax and Northern Ireland rates. 


\section{Table A22}

Household expenditure by number of persons working, 2008

based on weighted data and including children's expenditure

\begin{tabular}{|c|c|c|c|c|c|c|}
\hline & \multicolumn{5}{|c|}{ Number of persons working } & \multirow{2}{*}{$\begin{array}{l}\text { Al } \\
\text { house } \\
\text { holds }\end{array}$} \\
\hline & None & One & Two & Three & $\begin{array}{r}\text { Four } \\
\text { or more }\end{array}$ & \\
\hline Weighted number of households (thousands) & 8,850 & 7,150 & 7,580 & 1,540 & 550 & 25,690 \\
\hline Total number of households in sample & 2,080 & 1,660 & 1,710 & 300 & 100 & 5,850 \\
\hline Total number of persons in sample & 3,530 & 3,670 & 5,060 & 1,130 & 440 & 13,830 \\
\hline Total number of adults in sample & 3,030 & 2,660 & 3,630 & 920 & 390 & 10,640 \\
\hline Weighted average age of head of household & 66 & 47 & 42 & 49 & 48 & 52 \\
\hline \multicolumn{7}{|l|}{ Employment status of the household reference person': } \\
\hline - \% working full-time or self-employed & 0 & 67 & 88 & 89 & 87 & 51 \\
\hline - \% working part-time & 0 & 18 & 10 & 8 & 9 & 9 \\
\hline - \% not working & 100 & 14 & 2 & 2 & 2 & 40 \\
\hline
\end{tabular}

Commodity or service

Average weekly household expenditure (f)

\begin{tabular}{|c|c|c|c|c|c|c|c|}
\hline 1 & Food \& non-alcoholic drinks & 37.70 & 46.20 & 61.90 & 78.50 & 85.80 & 50.70 \\
\hline 2 & Alcoholic drinks, tobacco \& narcotics & 7.30 & 10.90 & 13.30 & 16.10 & 16.40 & 10.80 \\
\hline 3 & Clothing \& footwear & 10.10 & 20.00 & 29.50 & 42.50 & 58.10 & 21.60 \\
\hline 4 & Housing(net) ${ }^{2}$, fuel \& power & 38.40 & 61.00 & 58.40 & 66.50 & 73.60 & 53.00 \\
\hline 5 & Household goods \& services & 19.60 & 28.00 & 42.70 & 34.10 & 44.50 & 30.10 \\
\hline 6 & Health & 3.90 & 4.60 & 5.40 & 7.20 & 21.90 & 5.10 \\
\hline 8 & Communication & 7.00 & 11.80 & 15.40 & 19.10 & 26.60 & 12.00 \\
\hline 9 & Recreation \& culture & 40.60 & 48.80 & 81.00 & 99.90 & 120.10 & 60.10 \\
\hline 10 & Education & 0.80 & 7.00 & 10.90 & 8.20 & [11.20] & 6.20 \\
\hline 11 & Restaurants \& hotels & 16.70 & 33.60 & 55.30 & 72.30 & 90.40 & 37.70 \\
\hline 12 & Miscellaneous goods \& services & 19.30 & 32.60 & 51.10 & 54.70 & 70.30 & 35.60 \\
\hline 13 & Other expenditure items & 29.30 & 90.80 & 135.50 & 112.60 & 116.30 & 84.60 \\
\hline Tota & expenditure & 254.60 & 455.30 & 654.70 & 728.90 & 896.00 & 471.00 \\
\hline \multicolumn{8}{|c|}{ Average weekly expenditure per person (f) } \\
\hline \multicolumn{2}{|c|}{ Total expenditure } & 153.50 & 209.90 & 226.00 & 194.60 & 190.80 & 199.80 \\
\hline
\end{tabular}

Note: The commodity and service categories are not comparable to those in publications before 2001-02. Please see page xiii for symbols and conventions used in this report.

1 Excludes households where the household reference person was on a Government-supported training scheme.

2 Excluding mortgage interest payments, council tax and Northern Ireland rates. 


\section{Table A23}

\section{Household expenditure by age at which the household reference person completed continuous full-time education, 2008}

based on weighted data and including children's expenditure

\begin{tabular}{|c|c|c|c|c|c|c|c|}
\hline & & $\begin{array}{r}\text { Aged } 14 \\
\text { and } \\
\text { under }\end{array}$ & Aged 15 & Aged 16 & $\begin{array}{r}\text { Aged } 17 \\
\text { and } \\
\text { under } 19\end{array}$ & $\begin{array}{r}\text { Aged } 19 \\
\text { and } \\
\text { under } 22\end{array}$ & $\begin{array}{l}\text { Aged } 22 \\
\text { or over }\end{array}$ \\
\hline \multicolumn{2}{|r|}{ Weighted number of households (thousands) } & 430 & 3,790 & 6,560 & 4,020 & 2,840 & 2,990 \\
\hline \multicolumn{2}{|r|}{ Total number of households in sample } & 110 & 910 & 1,490 & 920 & 640 & 630 \\
\hline \multicolumn{2}{|c|}{ Total number of persons in sample } & 250 & 1,980 & 4,130 & 2,450 & 1,660 & 1,670 \\
\hline \multicolumn{2}{|c|}{ Total number of adults in sample } & 190 & 1,710 & 2,870 & 1,690 & 1,220 & 1,220 \\
\hline \multicolumn{2}{|r|}{ Weighted average number of persons per household } & 2.6 & 2.2 & 2.8 & 2.6 & 2.5 & 2.6 \\
\hline \multicolumn{2}{|r|}{ Weighted average age of head of household } & 51 & 56 & 45 & 43 & 43 & 42 \\
\hline \multicolumn{2}{|c|}{ Commodity or service } & \multicolumn{6}{|c|}{ Average weekly household expenditure $(f)$} \\
\hline 1 & Food \& non-alcoholic drinks & 44.30 & 48.80 & 53.70 & 53.90 & 55.10 & 60.00 \\
\hline 2 & Alcoholic drinks, tobacco \& narcotics & 8.80 & 13.40 & 12.80 & 10.60 & 10.70 & 12.80 \\
\hline 3 & Clothing \& footwear & 19.30 & 16.30 & 25.60 & 26.50 & 29.00 & 28.10 \\
\hline 5 & Household goods \& services & 23.80 & 23.50 & 28.70 & 34.70 & 44.10 & 42.20 \\
\hline 6 & Health & 1.70 & 4.60 & 4.30 & 5.70 & 7.70 & 5.40 \\
\hline 7 & Transport & 37.50 & 52.40 & 67.50 & 78.30 & 91.60 & 96.00 \\
\hline 8 & Communication & 8.30 & 10.60 & 13.40 & 14.00 & 14.50 & 15.20 \\
\hline 9 & Recreation \& culture & 49.80 & 55.60 & 65.80 & 72.30 & 71.20 & 70.80 \\
\hline 10 & Education & {$[2.70]$} & 1.90 & 3.30 & 5.80 & 11.90 & 23.20 \\
\hline 11 & Restaurants \& hotels & 21.70 & 30.10 & 38.50 & 45.50 & 61.10 & 53.30 \\
\hline 12 & Miscellaneous goods \& services & 21.60 & 24.50 & 34.60 & 45.70 & 47.60 & 55.00 \\
\hline $1-12$ & All expenditure groups & 296.50 & 327.50 & 398.40 & 452.40 & 506.50 & 539.40 \\
\hline 13 & Other expenditure items & 48.50 & 47.80 & 85.90 & 107.30 & 123.30 & 159.50 \\
\hline \multicolumn{8}{|c|}{ Average weekly expenditure per person (f) } \\
\hline \multicolumn{2}{|c|}{ Total expenditure } & 133.30 & 171.00 & 175.30 & 212.90 & 250.30 & 271.00 \\
\hline
\end{tabular}

Note: The commodity and service categories are not comparable to those in publications before 2001-02. Please see page xiii for symbols and conventions used in this report.

1 Excluding mortgage interest payments, council tax and Northern Ireland rates. 


\section{Table A24}

\section{Household expenditure by socio-economic classification of the household reference person, 2008}

based on weighted data and including children's expenditure

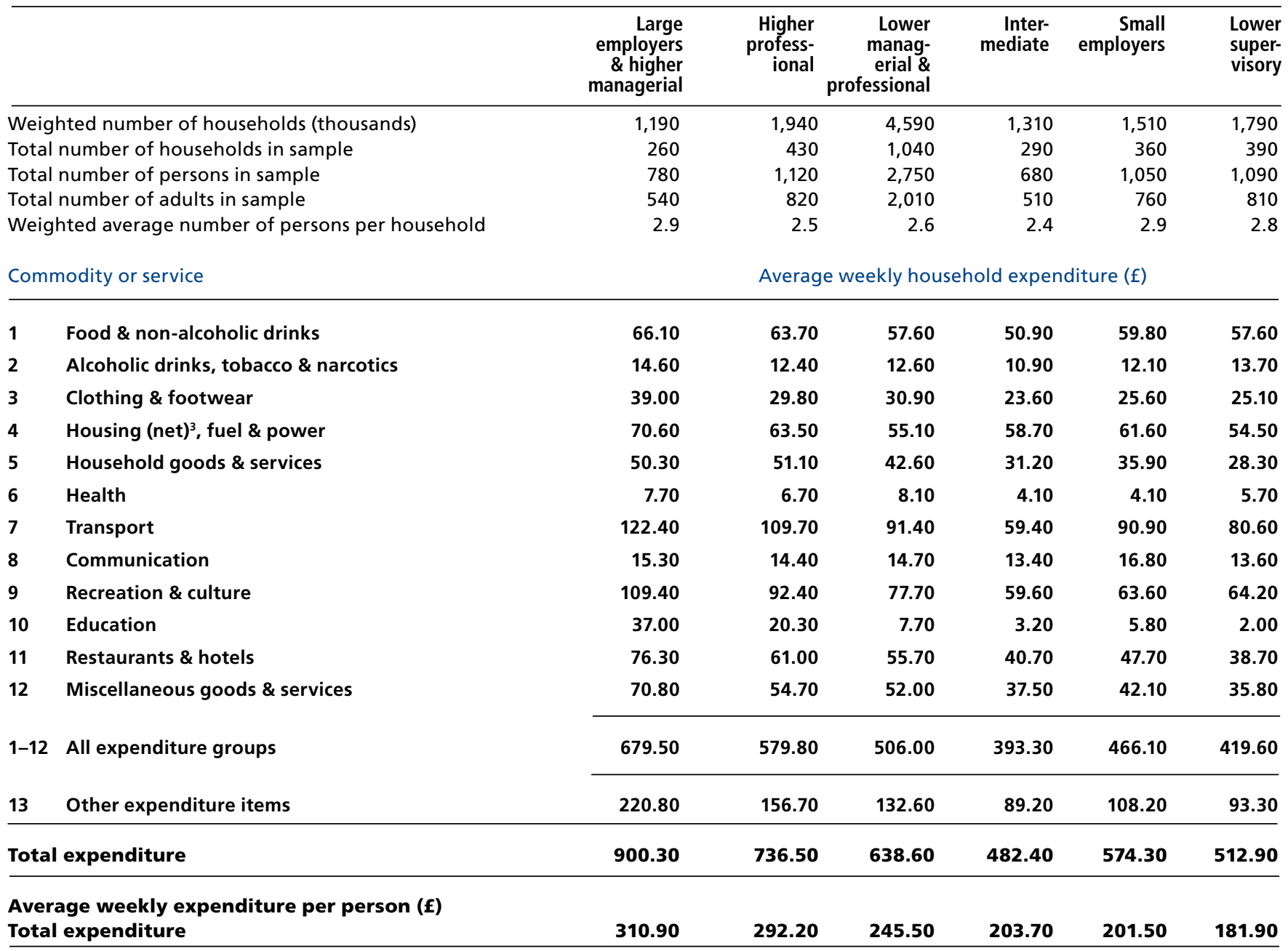

Note: The commodity and service categories are not comparable to those in publications before 2001-02.

Please see page xiii for symbols and conventions used in this report.

1 Includes those who have never worked.

2 Includes those who are economically inactive.

3 Excludes mortgage interest payments, council tax and Northern Ireland rates. 


\section{Table A24}

\section{Household expenditure by socio-economic classification of the household reference person, 2008 (cont.)}

based on weighted data and including children's expenditure

\begin{tabular}{|c|c|c|c|c|c|c|}
\hline & $\begin{array}{l}\text { Semi- } \\
\text { routine }\end{array}$ & Routine & $\begin{array}{r}\text { Long- } \\
\text { term } \\
\text { unem- } \\
\text { ployed }{ }^{1}\end{array}$ & Students & $\begin{array}{l}\text { Occupation } \\
\text { not stated }\end{array}$ & $\begin{array}{r}\text { All } \\
\text { house- } \\
\text { holds }\end{array}$ \\
\hline Weighted number of households (thousands) & 1,930 & 1,700 & 520 & 320 & 8,900 & 25,690 \\
\hline Total number of households in sample & 420 & 380 & 120 & 60 & 2,080 & 5,850 \\
\hline Total number of persons in sample & 1,150 & 1,010 & 350 & 160 & 3,680 & 13,830 \\
\hline Total number of adults in sample & 820 & 740 & 190 & 120 & 3,320 & 10,640 \\
\hline
\end{tabular}

Commodity or service

Average weekly household expenditure ( $f$ )

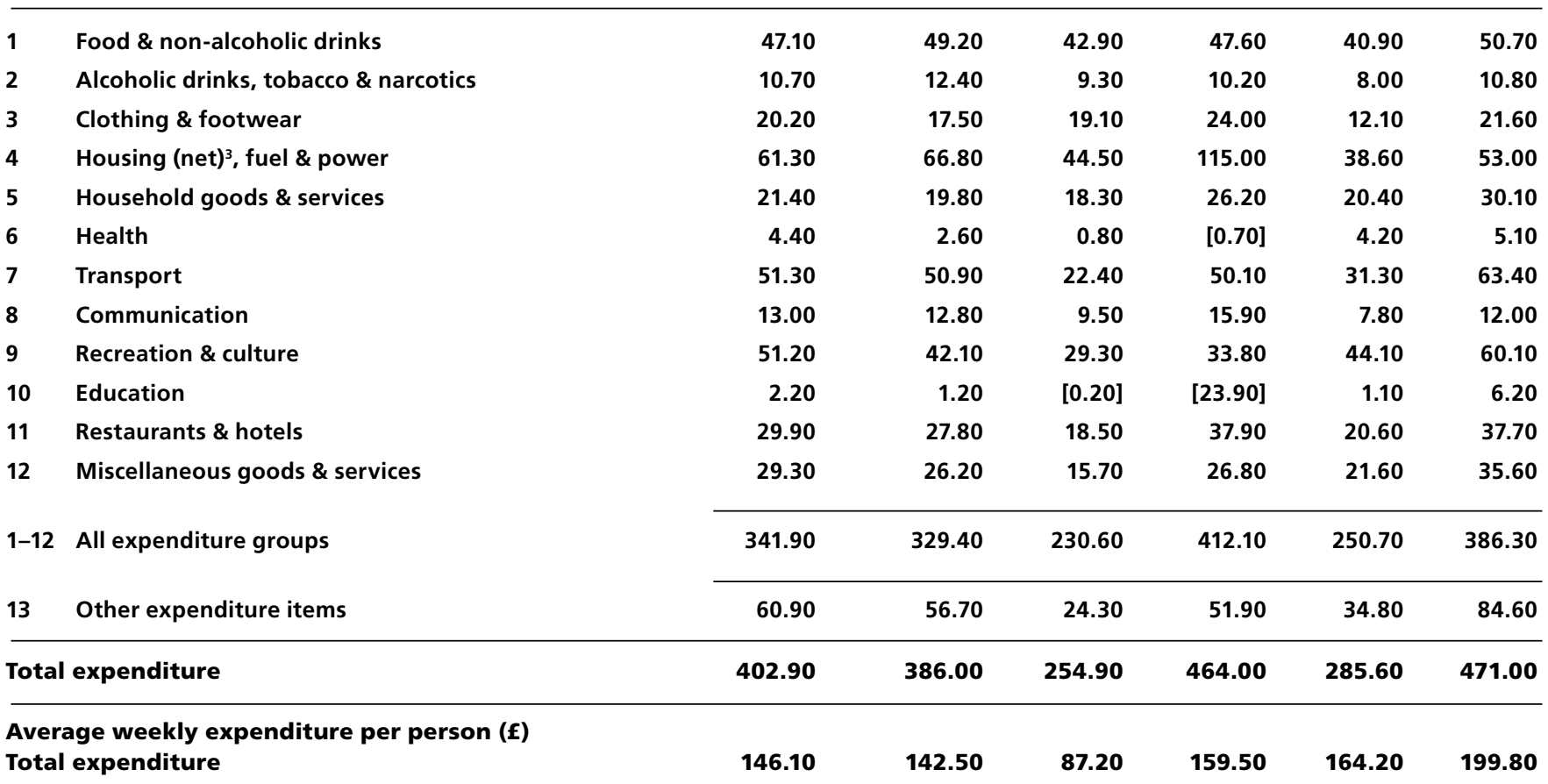

Note: The commodity and service categories are not comparable to those in publications before 2001-02.

Please see page xiii for symbols and conventions used in this report.

1 Includes those who have never worked

2 Includes those who are economically inactive.

3 Excludes mortgage interest payments, council tax and Northern Ireland rates. 


\section{Table A25}

\section{Expenditure by household composition, 2008}

based on weighted data and including children's expenditure

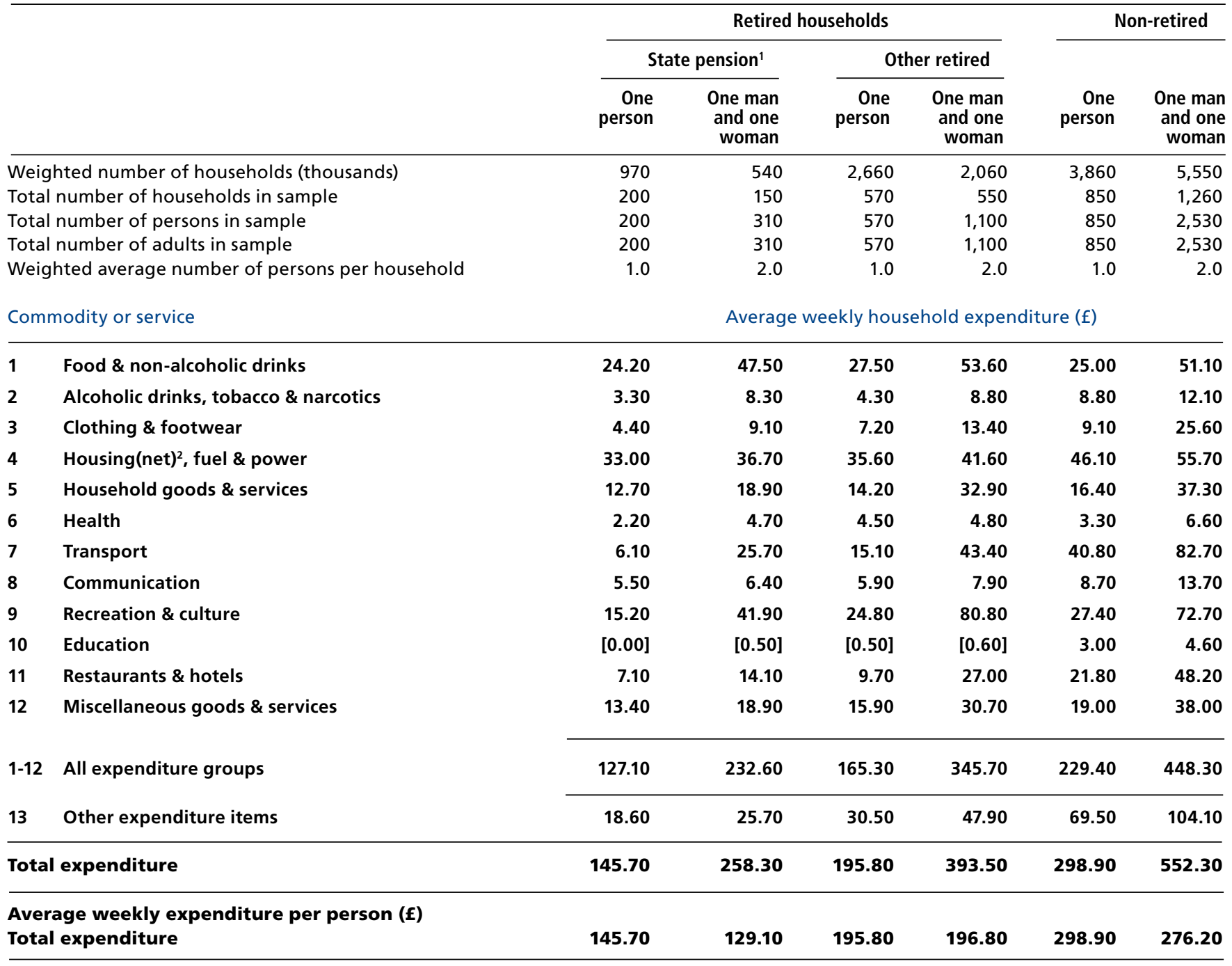

Note: The commodity and service categories are not comparable to those in publications before 2001-02. Please see page xiii for symbols and conventions used in this report.

1 Mainly dependent on state pensions and not economically active - see definitions in Appendix B.

2 Excluding mortgage interest payments, council tax and Northern Ireland rates. 


\section{Table A25}

\section{Expenditure by household composition, 2008 (cont.)}

based on weighted data and including children's expenditure

\begin{tabular}{|c|c|c|c|c|c|c|c|c|}
\hline & & \multicolumn{7}{|c|}{ Retired and non-retired households } \\
\hline & & \multicolumn{2}{|c|}{ One adult } & \multicolumn{3}{|c|}{ Two adults } & \multicolumn{2}{|c|}{ Three or more adults } \\
\hline & & $\begin{array}{l}\text { with } \\
\text { one } \\
\text { child }\end{array}$ & $\begin{array}{r}\text { with } \\
\text { two } \\
\text { or more } \\
\text { children }\end{array}$ & $\begin{array}{l}\text { with } \\
\text { one } \\
\text { child }\end{array}$ & $\begin{array}{r}\text { with } \\
\text { two } \\
\text { children }\end{array}$ & $\begin{array}{r}\text { with } \\
\text { three } \\
\text { or more } \\
\text { children }\end{array}$ & $\begin{array}{l}\text { without } \\
\text { children }\end{array}$ & $\begin{array}{r}\text { with } \\
\text { children }\end{array}$ \\
\hline \multicolumn{2}{|c|}{ Weighted number of households (thousands) } & 830 & 650 & 1,950 & 2,170 & 820 & 2,480 & 1,110 \\
\hline \multicolumn{2}{|c|}{ Total number of households in sample } & 200 & 180 & 430 & 540 & 210 & 460 & 240 \\
\hline \multicolumn{2}{|c|}{ Total number of persons in sample } & 400 & 610 & 1,300 & 2,170 & 1,110 & 1,560 & 1,140 \\
\hline \multirow{2}{*}{\multicolumn{2}{|c|}{$\begin{array}{l}\text { Total number of adults in sample } \\
\text { Weighted average number of persons per household }\end{array}$}} & 200 & 180 & 860 & 1,080 & 420 & 1,560 & 780 \\
\hline & & 2.0 & 3.4 & 3.0 & 4.0 & 5.3 & 3.4 & 4.9 \\
\hline \multicolumn{4}{|c|}{ Commodity or service } & \multicolumn{5}{|c|}{ Average weekly household expenditure (f) } \\
\hline 1 & Food \& non-alcoholic drinks & 37.10 & 50.10 & 62.60 & 72.30 & 84.40 & 73.50 & 84.80 \\
\hline 2 & Alcoholic drinks, tobacco \& narcotics & 9.00 & 8.10 & 13.90 & 13.80 & 11.50 & 16.70 & 16.10 \\
\hline 3 & Clothing \& footwear & 14.50 & 25.60 & 27.50 & 32.80 & 35.60 & 35.50 & 44.20 \\
\hline 4 & Housing(net) ${ }^{2}$, fuel \& power & 54.20 & 53.80 & 60.90 & 55.60 & 91.60 & 70.60 & 64.90 \\
\hline 5 & Household goods \& services & 16.80 & 25.90 & 37.10 & 42.80 & 57.90 & 36.40 & 36.70 \\
\hline 6 & Health & 2.70 & 2.40 & 3.40 & 4.80 & 3.60 & 11.00 & 4.00 \\
\hline 7 & Transport & 27.10 & 27.80 & 82.80 & 93.20 & 83.10 & 111.70 & 99.80 \\
\hline 8 & Communication & 9.70 & 11.10 & 14.00 & 14.10 & 16.50 & 18.60 & 21.20 \\
\hline 9 & Recreation \& culture & 32.60 & 43.60 & 69.30 & 89.20 & 81.80 & 88.60 & 81.40 \\
\hline 10 & Education & [3.70] & 3.60 & 10.20 & 18.40 & 23.60 & 9.20 & 10.20 \\
\hline 11 & Restaurants \& hotels & 18.10 & 25.50 & 45.90 & 54.00 & 55.60 & 67.60 & 61.30 \\
\hline 12 & Miscellaneous goods \& services & 25.40 & 22.10 & 54.80 & 57.60 & 65.70 & 49.80 & 50.90 \\
\hline $1-12$ & All expenditure groups & 251.00 & 299.70 & 482.60 & 548.70 & 610.80 & 589.20 & 575.50 \\
\hline 13 & Other expenditure items & 49.80 & 40.00 & 116.90 & 140.80 & 191.20 & 95.60 & 107.30 \\
\hline \multicolumn{2}{|c|}{ Total expenditure } & 300.80 & 339.70 & 599.40 & 689.50 & 802.00 & 684.80 & 682.80 \\
\hline \multicolumn{9}{|c|}{ Average weekly expenditure per person (f) } \\
\hline
\end{tabular}

Note: The commodity and service categories are not comparable to those in publications before 2001-02. Please see page xiii for symbols and conventions used in this report.

1 Mainly dependent on state pensions and not economically active - see definitions in Appendix B.

2 Excluding mortgage interest payments, council tax and Northern Ireland rates. 


\section{Table A26}

\section{Expenditure of one person retired households mainly dependent on state pensions ${ }^{1}$ by gross income quintile group, 2006-2008}

based on weighted data

\begin{tabular}{|c|c|c|c|c|c|c|c|}
\hline & & $\begin{array}{r}\text { Lowest } \\
\text { twenty } \\
\text { per cent }\end{array}$ & $\begin{array}{l}\text { Second } \\
\text { quintile } \\
\text { group }\end{array}$ & $\begin{array}{l}\text { Third } \\
\text { quintile } \\
\text { group }\end{array}$ & $\begin{array}{r}\text { Fourth } \\
\text { quintile } \\
\text { group }\end{array}$ & $\begin{array}{c}\text { Highest } \\
\text { twenty } \\
\text { per cent }\end{array}$ & $\begin{array}{r}\text { All } \\
\text { house- } \\
\text { holds }\end{array}$ \\
\hline \multicolumn{2}{|c|}{ Lower boundary of group ( $\mathrm{f}$ per week) ${ }^{2}$} & & 223 & 408 & 664 & 1026 & \\
\hline \multicolumn{2}{|r|}{ Average weighted number of households (thousands) } & 690 & 90 & - & 0 & 0 & 790 \\
\hline \multicolumn{2}{|r|}{ Total number of households in sample (over 3 years) } & 480 & 60 & - & 0 & 0 & 540 \\
\hline \multicolumn{2}{|c|}{ Total number of persons in sample (over 3 years) } & 480 & 60 & - & 0 & 0 & 540 \\
\hline \multirow{2}{*}{\multicolumn{2}{|c|}{$\begin{array}{l}\text { Total number of adults in sample (over } 3 \text { years) } \\
\text { Weighted average number of persons per household }\end{array}$}} & 480 & 60 & - & 0 & 0 & 540 \\
\hline & & 1.0 & 1.0 & 1.0 & 0 & 0 & 1.0 \\
\hline \multicolumn{2}{|c|}{ Commodity or service } & \multicolumn{6}{|c|}{ Average weekly household expenditure $(f)$} \\
\hline 1 & Food \& non-alcoholic drinks & 23.50 & 21.70 & [18.10] & - & - & 23.30 \\
\hline 2 & Alcoholic drinks, tobacco \& narcotics & 3.10 & {$[2.70]$} & {$[1.60]$} & - & - & 3.00 \\
\hline 3 & Clothing \& footwear & 4.70 & 6.70 & {$[3.00]$} & - & - & 4.80 \\
\hline 4 & Housing(net) ${ }^{3}$, fuel \& power & 30.50 & 34.30 & [33.10] & - & - & 31.00 \\
\hline 5 & Household goods \& services & 13.30 & 15.10 & [3.50] & - & - & 13.70 \\
\hline 6 & Health & 1.50 & 6.40 & [1.50] & - & - & 2.10 \\
\hline 7 & Transport & 9.00 & 6.40 & [1.90] & - & - & 8.80 \\
\hline 8 & Communication & 4.90 & 4.90 & {$[2.20]$} & - & - & 4.80 \\
\hline 9 & Recreation \& culture & 18.50 & 14.70 & {$[9.70]$} & - & - & 18.20 \\
\hline 10 & Education & - & - & - & - & - & - \\
\hline 11 & Restaurants \& hotels & 6.80 & 7.50 & {$[2.70]$} & - & - & {$[6.80]$} \\
\hline 12 & Miscellaneous goods \& services & 12.70 & 15.60 & [8.10] & - & - & 13.00 \\
\hline $1-12$ & All expenditure groups & 128.50 & 136.10 & [85.30] & - & - & 129.60 \\
\hline 13 & Other expenditure items & 17.80 & 33.40 & [5.60] & - & - & 19.10 \\
\hline \multicolumn{2}{|c|}{ Total expenditure } & 146.30 & 169.50 & [90.90] & - & - & 148.70 \\
\hline \multirow{2}{*}{\multicolumn{2}{|c|}{$\begin{array}{l}\text { Average weekly expenditure per person (f) } \\
\text { Total expenditure }\end{array}$}} & & & & & & \\
\hline & & 146.30 & 169.50 & [90.90] & - & - & 148.70 \\
\hline
\end{tabular}

Note: The commodity and service categories are not comparable to those in publications before 2001-02.

Please see page xiii for symbols and conventions used in this report.

This table is based on a three year average.

1 Mainly dependent on state pensions and not economically active - see defintions in Appendix B.

2 Lower boundary of 2008 gross income quintile groups ( $f$ per week).

3 Excluding mortgage interest payments, council tax and Northern Ireland rates. 


\section{Table A27}

Expenditure of one person retired households not mainly dependent on state pensions by gross income quintile group, 2006-2008

based on weighted data

\begin{tabular}{|c|c|c|c|c|c|c|c|}
\hline & & $\begin{array}{r}\text { Lowest } \\
\text { twenty } \\
\text { per cent }\end{array}$ & $\begin{array}{r}\text { Second } \\
\text { quintile } \\
\text { group }\end{array}$ & $\begin{array}{r}\text { Third } \\
\text { quintile } \\
\text { group }\end{array}$ & $\begin{array}{r}\text { Fourth } \\
\text { quintile } \\
\text { group }\end{array}$ & $\begin{array}{r}\text { Highest } \\
\text { twenty } \\
\text { per cent }\end{array}$ & $\begin{array}{r}\text { All } \\
\text { house- } \\
\text { holds }\end{array}$ \\
\hline \multicolumn{2}{|c|}{ Lower boundary of group ( $\mathrm{f}$ per week) ${ }^{1}$} & & 223 & 408 & 664 & 1026 & \\
\hline \multicolumn{2}{|r|}{ Average weighted number of households (thousands) } & 1,550 & 860 & 220 & 70 & 10 & 2,710 \\
\hline \multicolumn{2}{|r|}{ Total number of households in sample (over 3 years) } & 1,060 & 600 & 160 & 50 & 10 & 1,870 \\
\hline \multicolumn{2}{|r|}{ Total number of persons in sample (over 3 years) } & 1,060 & 600 & 160 & 50 & 10 & 1,870 \\
\hline \multicolumn{2}{|r|}{ Weighted average number of persons per household } & 1.0 & 1.0 & 1.0 & 1.0 & 1.0 & 1.0 \\
\hline \multicolumn{2}{|c|}{ Commodity or service } & \multicolumn{6}{|c|}{ Average weekly household expenditure $(f)$} \\
\hline 1 & Food \& non-alcoholic drinks & 24.40 & 27.30 & 33.10 & 32.70 & {$[17.00]$} & 26.20 \\
\hline 2 & Alcoholic drinks, tobacco \& narcotics & 3.80 & 4.80 & 7.30 & 7.30 & {$[3.50]$} & 4.50 \\
\hline 3 & Clothing \& footwear & 5.60 & 7.30 & 10.10 & 16.00 & [10.20] & 6.70 \\
\hline 5 & Household goods \& services & 11.30 & 17.60 & 31.90 & 38.70 & {$[7.90]$} & 15.50 \\
\hline 6 & Health & 3.10 & 5.60 & 9.90 & 8.50 & {$[0.30]$} & 4.50 \\
\hline 7 & Transport & 7.90 & 18.90 & 41.00 & 49.50 & [39.40] & 15.30 \\
\hline 8 & Communication & 5.00 & 5.80 & 7.80 & 8.50 & [3.30] & 5.60 \\
\hline 9 & Recreation \& culture & 15.20 & 26.90 & 52.60 & 52.60 & {$[171.80]$} & 23.70 \\
\hline 10 & Education & {$[0.00]$} & {$[0.40]$} & {$[4.30]$} & {$[20.80]$} & - & 1.00 \\
\hline 11 & Restaurants \& hotels & 6.70 & 11.70 & 17.00 & 33.90 & {$[17.60]$} & 9.90 \\
\hline 12 & Miscellaneous goods \& services & 10.20 & 19.60 & 41.40 & 47.40 & {$[18.10]$} & 16.60 \\
\hline $1-12$ & All expenditure groups & 123.80 & 181.20 & 297.90 & 368.00 & [309.10] & 163.10 \\
\hline 13 & Other expenditure items & 13.90 & 30.00 & 51.80 & 165.80 & {$[77.50]$} & 26.00 \\
\hline \multicolumn{8}{|c|}{ Average weekly expenditure per person (f) } \\
\hline \multicolumn{2}{|c|}{ Total expenditure } & 137.70 & 211.20 & 349.70 & 533.80 & [579.90] & 189.10 \\
\hline
\end{tabular}

Note: The commodity and service categories are not comparable to those in publications before 2001-02.

Please see page xiii for symbols and conventions used in this report.

This table is based on a three year average.

1 Lower boundary of 2008 gross income quintile groups ( $f$ per week).

2 Excluding mortgage interest payments, council tax and Northern Ireland rates. 


\section{Table A28}

\section{Expenditure of one adult non-retired households by gross income quintile group, 2006-2008}

based on weighted data

\begin{tabular}{|c|c|c|c|c|c|c|c|}
\hline & & $\begin{array}{l}\text { Lowest } \\
\text { twenty } \\
\text { per cent }\end{array}$ & $\begin{array}{l}\text { Second } \\
\text { quintile } \\
\text { group }\end{array}$ & $\begin{array}{l}\text { Third } \\
\text { quintile } \\
\text { group }\end{array}$ & $\begin{array}{r}\text { Fourth } \\
\text { quintile } \\
\text { group }\end{array}$ & $\begin{array}{r}\text { Highest } \\
\text { twenty } \\
\text { per cent }\end{array}$ & $\begin{array}{r}\text { All } \\
\text { house- } \\
\text { holds }\end{array}$ \\
\hline \multicolumn{2}{|c|}{ Lower boundary of group ( $\mathrm{f}$ per week) ${ }^{1}$} & & 223 & 408 & 664 & 1026 & \\
\hline \multicolumn{2}{|r|}{ Average weighted number of households (thousands) } & 1,210 & 950 & 900 & 500 & 270 & 3,830 \\
\hline \multicolumn{2}{|r|}{ Total number of households in sample (over 3 years) } & 850 & 680 & 630 & 340 & 170 & 2,670 \\
\hline \multicolumn{2}{|r|}{ Total number of persons in sample (over 3 years) } & 850 & 680 & 630 & 340 & 170 & 2,670 \\
\hline \multicolumn{2}{|c|}{ Weighted average number of persons per household } & 1.0 & 1.0 & 1.0 & 1.0 & 1.0 & 1.0 \\
\hline \multicolumn{2}{|c|}{ Commodity or service } & \multicolumn{6}{|c|}{ Average weekly household expenditure (f) } \\
\hline 1 & Food \& non-alcoholic drinks & 19.90 & 23.30 & 25.90 & 31.10 & 30.20 & 24.30 \\
\hline 2 & Alcoholic drinks, tobacco \& narcotics & 7.50 & 7.50 & 8.00 & 9.60 & 8.00 & 7.90 \\
\hline 3 & Clothing \& footwear & 5.50 & 8.50 & 10.80 & 16.00 & 23.70 & 10.20 \\
\hline 4 & Housing(net) ${ }^{2}$, fuel \& power & 35.90 & 49.70 & 44.20 & 50.30 & 60.40 & 45.00 \\
\hline 5 & Household goods \& services & 9.50 & 14.80 & 18.90 & 34.10 & 43.10 & 18.70 \\
\hline 6 & Health & 1.50 & 2.90 & 2.70 & 7.00 & 4.60 & 3.10 \\
\hline 7 & Transport & 16.80 & 34.30 & 46.60 & 58.60 & 98.70 & 39.50 \\
\hline 8 & Communication & 6.40 & 8.50 & 9.70 & 10.50 & 12.90 & 8.70 \\
\hline 9 & Recreation \& culture & 16.40 & 26.90 & 36.60 & 47.50 & 57.10 & 30.80 \\
\hline 10 & Education & 1.40 & {$[0.80]$} & 1.00 & 11.70 & [7.40] & 2.90 \\
\hline 11 & Restaurants \& hotels & 10.50 & 17.20 & 29.80 & 36.70 & 56.40 & 23.40 \\
\hline 12 & Miscellaneous goods \& services & 10.50 & 17.60 & 22.70 & 36.10 & 39.80 & 20.60 \\
\hline $1-12$ & All expenditure groups & 141.70 & 211.80 & 256.90 & 349.10 & 442.20 & 235.00 \\
\hline 13 & Other expenditure items & 18.30 & 52.80 & 78.90 & 110.00 & 194.70 & 65.50 \\
\hline \multicolumn{8}{|c|}{ Average weekly expenditure per person (f) } \\
\hline \multicolumn{2}{|c|}{ Total expenditure } & 160.10 & 264.60 & 335.70 & 459.10 & 636.90 & 300.50 \\
\hline
\end{tabular}

Note: The commodity and service categories are not comparable to those in publications before 2001-02.

Please see page xiii for symbols and conventions used in this report.

This table is based on a three year average.

1 Lower boundary of 2008 gross income quintile groups ( $\mathrm{f}$ per week).

2 Excluding mortgage interest payments, council tax and Northern Ireland rates. 


\section{Table A29}

\section{Expenditure of one adult households with children by gross income quintile group, 2006-2008}

based on weighted data and including children's expenditure

\begin{tabular}{|c|c|c|c|c|c|c|c|}
\hline & & $\begin{array}{l}\text { Lowest } \\
\text { twenty } \\
\text { per cent }\end{array}$ & $\begin{array}{l}\text { Second } \\
\text { quintile } \\
\text { group }\end{array}$ & $\begin{array}{l}\text { Third } \\
\text { quintile } \\
\text { group }\end{array}$ & $\begin{array}{r}\text { Fourth } \\
\text { quintile } \\
\text { group }\end{array}$ & $\begin{array}{l}\text { Highest } \\
\text { twenty } \\
\text { per cent }\end{array}$ & $\begin{array}{r}\text { All } \\
\text { house- } \\
\text { holds }\end{array}$ \\
\hline \multicolumn{2}{|c|}{ Lower boundary of group ( $f$ per week) ${ }^{1}$} & & 223 & 408 & 664 & 1026 & \\
\hline \multirow{2}{*}{\multicolumn{2}{|c|}{ Average weighted number of households (thousands) }} & 600 & 460 & 250 & 120 & 30 & 1,460 \\
\hline & & 500 & 410 & 230 & 90 & 30 & 1,250 \\
\hline \multicolumn{2}{|r|}{ Total number of persons in sample (over 3 years) } & 1,290 & 1,150 & 630 & 230 & 80 & 3,370 \\
\hline \multicolumn{2}{|r|}{ Total number of adults in sample (over 3 years) } & 500 & 410 & 230 & 90 & 30 & 1,250 \\
\hline \multicolumn{2}{|r|}{ Weighted average number of persons per household } & 2.5 & 2.7 & 2.7 & 2.5 & 2.7 & 2.6 \\
\hline \multicolumn{2}{|c|}{ Commodity or service } & \multicolumn{6}{|c|}{ Average weekly household expenditure (f) } \\
\hline 1 & Food \& non-alcoholic drinks & 35.00 & 42.10 & 44.80 & 48.30 & 60.60 & 40.50 \\
\hline 2 & Alcoholic drinks, tobacco \& narcotics & 6.80 & 8.60 & 9.40 & 10.10 & [7.00] & 8.10 \\
\hline 3 & Clothing \& footwear & 12.70 & 21.00 & 29.80 & 30.60 & 49.70 & 20.50 \\
\hline 4 & Housing(net) ${ }^{2}$, fuel \& power & 42.40 & 55.30 & 56.90 & 58.80 & 100.80 & 51.30 \\
\hline 5 & Household goods \& services & 15.60 & 23.00 & 23.50 & 31.10 & 35.90 & 21.20 \\
\hline 6 & Health & 1.50 & 1.40 & 2.40 & 6.90 & [12.60] & 2.30 \\
\hline 7 & Transport & 14.10 & 28.30 & 46.20 & 52.20 & 72.40 & 28.30 \\
\hline 8 & Communication & 7.80 & 11.00 & 15.40 & 14.20 & 17.10 & 10.70 \\
\hline 9 & Recreation \& culture & 23.70 & 37.90 & 48.50 & 90.40 & 176.60 & 40.70 \\
\hline 10 & Education & 1.10 & 3.50 & 4.30 & [6.90] & [73.00] & 4.90 \\
\hline 11 & Restaurants \& hotels & 13.50 & 19.90 & 29.70 & 45.60 & 39.00 & 21.40 \\
\hline 12 & Miscellaneous goods \& services & 15.20 & 23.80 & 43.20 & 41.20 & 74.30 & 26.00 \\
\hline $1-12$ & All expenditure groups & 189.40 & 275.60 & 354.10 & 436.40 & 719.00 & 275.90 \\
\hline 13 & Other expenditure items & 11.30 & 45.20 & 79.40 & 106.70 & 194.10 & 44.90 \\
\hline \multicolumn{8}{|c|}{ Average weekly expenditure per person (f) } \\
\hline \multicolumn{2}{|c|}{ Total expenditure } & 79.10 & 117.20 & 160.60 & 219.80 & 341.10 & 122.20 \\
\hline
\end{tabular}

Note: The commodity and service categories are not comparable to those in publications before $2001-02$. Please see page xiii for symbols and conventions used in this report.

This table is based on a three year average.

1 Lower boundary of 2008 gross income quintile groups ( $\mathrm{f}$ per week).

2 Excluding mortgage interest payments, council tax and Northern Ireland rates. 


\section{Table A30}

\section{Expenditure of two adult households with children \\ by gross income quintile group, 2006-2008}

based on weighted data and including children's expenditure

\begin{tabular}{|c|c|c|c|c|c|c|c|}
\hline & & $\begin{array}{l}\text { Lowest } \\
\text { twenty } \\
\text { per cent }\end{array}$ & $\begin{array}{l}\text { Second } \\
\text { quintile } \\
\text { group }\end{array}$ & $\begin{array}{l}\text { Third } \\
\text { quintile } \\
\text { group }\end{array}$ & $\begin{array}{r}\text { Fourth } \\
\text { quintile } \\
\text { group }\end{array}$ & $\begin{array}{l}\text { Highest } \\
\text { twenty } \\
\text { per cent }\end{array}$ & $\begin{array}{r}\text { All } \\
\text { house- } \\
\text { holds }\end{array}$ \\
\hline \multicolumn{2}{|c|}{ Lower boundary of group ( $f$ per week) ${ }^{1}$} & & 223 & 408 & 664 & 1026 & \\
\hline \multicolumn{2}{|r|}{ Average weighted number of households (thousands) } & 230 & 550 & 1,110 & 1,490 & 1,530 & 4,920 \\
\hline \multicolumn{2}{|r|}{ Total number of households in sample (over 3 years) } & 160 & 420 & 870 & 1,170 & 1,200 & 3,800 \\
\hline \multicolumn{2}{|r|}{ Total number of persons in sample (over 3 years) } & 560 & 1,690 & 3,390 & 4,460 & 4,630 & 14,730 \\
\hline \multicolumn{2}{|r|}{ Total number of adults in sample (over 3 years) } & 310 & 840 & 1,730 & 2,330 & 2,390 & 7,600 \\
\hline \multicolumn{2}{|r|}{ Weighted average number of persons per household } & 3.7 & 4.0 & 3.8 & 3.8 & 3.8 & 3.8 \\
\hline \multicolumn{2}{|c|}{ Commodity or service } & \multicolumn{6}{|c|}{ Average weekly household expenditure (f) } \\
\hline 1 & Food \& non-alcoholic drinks & 48.60 & 53.20 & 59.30 & 65.20 & 80.80 & 66.60 \\
\hline 2 & Alcoholic drinks, tobacco \& narcotics & 13.70 & 12.50 & 12.00 & 12.40 & 15.00 & 13.20 \\
\hline 3 & Clothing \& footwear & 15.10 & 25.80 & 23.40 & 31.60 & 45.70 & 32.70 \\
\hline 4 & Housing(net) ${ }^{2}$, fuel \& power & 46.00 & 60.10 & 59.40 & 51.30 & 63.50 & 57.70 \\
\hline 5 & Household goods \& services & 18.50 & 26.20 & 27.00 & 38.70 & 62.80 & 41.20 \\
\hline 6 & Health & 2.40 & 1.60 & 3.50 & 3.90 & 9.10 & 5.10 \\
\hline 7 & Transport & 31.40 & 45.10 & 58.90 & 77.70 & 139.50 & 86.80 \\
\hline 8 & Communication & 9.30 & 12.60 & 14.30 & 14.50 & 16.90 & 14.80 \\
\hline 9 & Recreation \& culture & 28.20 & 42.90 & 55.20 & 78.70 & 114.50 & 78.20 \\
\hline 10 & Education & [7.30] & 3.30 & 4.20 & 5.80 & 42.30 & 16.60 \\
\hline 11 & Restaurants \& hotels & 25.20 & 30.60 & 32.40 & 46.10 & 72.90 & 48.70 \\
\hline 12 & Miscellaneous goods \& services & 18.60 & 27.50 & 38.30 & 51.80 & 83.20 & 54.20 \\
\hline $1-12$ & All expenditure groups & 264.20 & 341.40 & 387.80 & 477.90 & 746.30 & 515.70 \\
\hline 13 & Other expenditure items & 33.30 & 52.00 & 76.70 & 121.40 & 212.00 & 127.60 \\
\hline \multicolumn{8}{|c|}{ Average weekly expenditure per person (f) } \\
\hline Tota & expenditure & 81.50 & 99.00 & 121.40 & 158.80 & 250.20 & 168.40 \\
\hline
\end{tabular}

Note: $\quad$ The commodity and service categories are not comparable to those in publications before 2001-02.

Please see page xiii for symbols and conventions used in this report.

This table is based on a three year average.

1 Lower boundary of 2008 gross income quintile groups ( $f$ per week).

2 Excluding mortgage interest payments, council tax and Northern Ireland rates. 


\section{Table A31}

\section{Expenditure of one man one woman non-retired households by gross income quintile group, 2006-2008}

based on weighted data

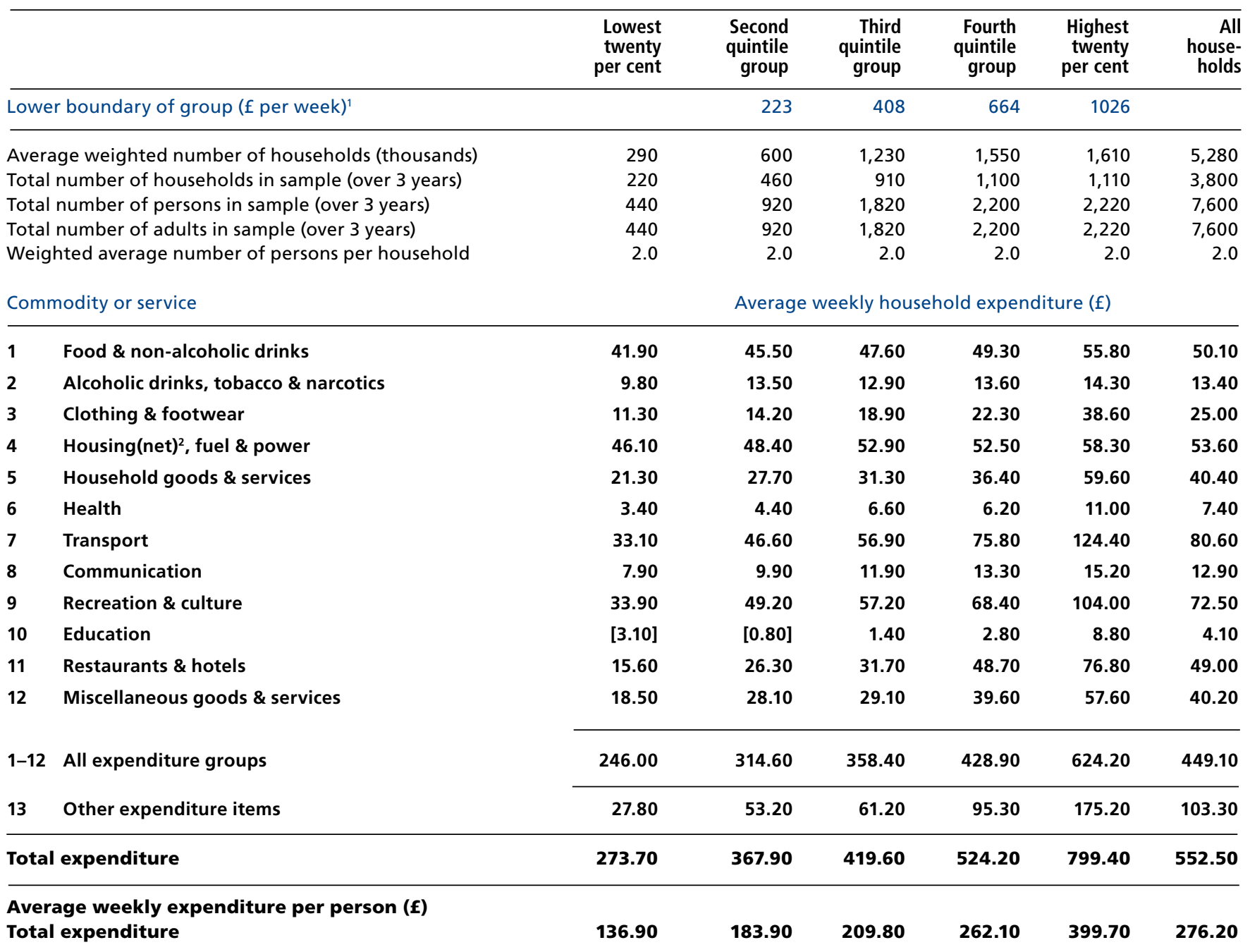

Note: The commodity and service categories are not comparable to those in publications before 2001-02 Please see page xiii for symbols and conventions used in this report.

This table is based on a three year average.

1 Lower boundary of 2008 gross income quintile groups ( $f$ per week).

2 Excluding mortgage interest payments, council tax and Northern Ireland rates. 


\section{Table A32}

\section{Expenditure of one man one woman retired households mainly} dependent on state pensions ${ }^{1}$ by gross income quintile group, 2006-2008 based on weighted data

\begin{tabular}{|c|c|c|c|c|c|c|c|}
\hline & & $\begin{array}{r}\text { Lowest } \\
\text { twenty } \\
\text { per cent }\end{array}$ & $\begin{array}{l}\text { Second } \\
\text { quintile } \\
\text { group }\end{array}$ & $\begin{array}{l}\text { Third } \\
\text { quintile } \\
\text { group }\end{array}$ & $\begin{array}{r}\text { Fourth } \\
\text { quintile } \\
\text { group }\end{array}$ & $\begin{array}{c}\text { Highest } \\
\text { twenty } \\
\text { per cent }\end{array}$ & $\begin{array}{r}\text { All } \\
\text { house- } \\
\text { holds }\end{array}$ \\
\hline \multicolumn{2}{|c|}{ Lower boundary of group ( $\mathrm{f}$ per week) ${ }^{2}$} & & 223 & 408 & 664 & 1026 & \\
\hline \multicolumn{2}{|r|}{ Average weighted number of households (thousands) } & 200 & 270 & 10 & 0 & 0 & 490 \\
\hline \multicolumn{2}{|r|}{ Total number of households in sample (over 3 years) } & 170 & 240 & 10 & 0 & 0 & 430 \\
\hline \multicolumn{2}{|c|}{ Total number of persons in sample (over 3 years) } & 350 & 480 & 20 & 0 & 0 & 850 \\
\hline \multirow{2}{*}{\multicolumn{2}{|c|}{$\begin{array}{l}\text { Total number of adults in sample (over } 3 \text { years) } \\
\text { Weighted average number of persons per household }\end{array}$}} & 350 & 480 & 20 & 0 & 0 & 850 \\
\hline & & 2.0 & 2.0 & 2.0 & 0 & 0 & 2.0 \\
\hline \multicolumn{2}{|c|}{ Commodity or service } & \multicolumn{6}{|c|}{ Average weekly household expenditure $(f)$} \\
\hline 1 & Food \& non-alcoholic drinks & 43.90 & 45.20 & [47.40] & - & - & 44.70 \\
\hline 2 & Alcoholic drinks, tobacco \& narcotics & 5.70 & 8.70 & [10.10] & - & - & 7.50 \\
\hline 3 & Clothing \& footwear & 8.30 & 9.80 & [7.70] & - & - & 9.10 \\
\hline 4 & Housing(net) ${ }^{3}$, fuel \& power & 33.50 & 35.60 & [41.50] & - & - & 35.10 \\
\hline 5 & Household goods \& services & 11.80 & 19.30 & {$[8.60]$} & - & - & 15.90 \\
\hline 6 & Health & 2.40 & 3.90 & [7.00] & - & - & 3.40 \\
\hline 7 & Transport & 23.80 & 28.90 & [26.10] & - & - & 26.80 \\
\hline 8 & Communication & 6.10 & 6.30 & [7.40] & - & - & 6.20 \\
\hline 9 & Recreation \& culture & 30.10 & 40.00 & [37.00] & - & - & 35.80 \\
\hline 10 & Education & - & [0.30] & - & - & - & 0.20 \\
\hline 11 & Restaurants \& hotels & 12.20 & 15.00 & [19.10] & - & - & 13.90 \\
\hline 12 & Miscellaneous goods \& services & 17.40 & 18.70 & [15.60] & - & - & 18.00 \\
\hline $1-12$ & All expenditure groups & 195.30 & 231.70 & [227.50] & - & - & 216.80 \\
\hline 13 & Other expenditure items & 27.90 & 28.30 & [52.20] & - & - & 28.30 \\
\hline \multicolumn{2}{|c|}{ Total expenditure } & 223.20 & 260.10 & [279.60] & - & - & 245.10 \\
\hline \multirow{2}{*}{\multicolumn{2}{|c|}{$\begin{array}{l}\text { Average weekly expenditure per person (f) } \\
\text { Total expenditure }\end{array}$}} & & & & & & \\
\hline & & 111.60 & 130.00 & [139.80] & - & - & 122.50 \\
\hline
\end{tabular}

Note: The commodity and service categories are not comparable to those in publications before 2001-02. Please see page xiii for symbols and conventions used in this report.

This table is based on a three year average

1 Mainly dependent on the state pensions and not economically active -see defintions in Appendix B.

2 Lower boundary of 2008 gross income quintile groups (f per week).

3 Excluding mortgage interest payments, council tax and Northern Ireland rates. 


\section{Table A33}

Expenditure of one man one woman retired households not mainly dependent on state pensions by gross income quintile group, 2006-2008 based on weighted data

\begin{tabular}{|c|c|c|c|c|c|c|c|}
\hline & & $\begin{array}{r}\text { Lowest } \\
\text { twenty } \\
\text { per cent }\end{array}$ & $\begin{array}{r}\text { Second } \\
\text { quintile } \\
\text { group }\end{array}$ & $\begin{array}{r}\text { Third } \\
\text { quintile } \\
\text { group }\end{array}$ & $\begin{array}{r}\text { Fourth } \\
\text { quintile } \\
\text { group }\end{array}$ & $\begin{array}{r}\text { Highest } \\
\text { twenty } \\
\text { per cent }\end{array}$ & $\begin{array}{r}\text { All } \\
\text { house- } \\
\text { holds }\end{array}$ \\
\hline \multicolumn{2}{|c|}{ Lower boundary of group ( $\mathrm{f}$ per week) ${ }^{1}$} & & 223 & 408 & 664 & 1026 & \\
\hline \multicolumn{2}{|r|}{ Average weighted number of households (thousands) } & 170 & 960 & 610 & 240 & 100 & 2,080 \\
\hline \multicolumn{2}{|r|}{ Total number of households in sample (over 3 years) } & 130 & 800 & 510 & 210 & 90 & 1,730 \\
\hline \multicolumn{2}{|c|}{ Total number of persons in sample (over 3 years) } & 250 & 1,600 & 1,020 & 410 & 170 & 3,450 \\
\hline \multirow{2}{*}{\multicolumn{2}{|c|}{$\begin{array}{l}\text { Total number of adults in sample (over } 3 \text { years) } \\
\text { Weighted average number of persons per household }\end{array}$}} & 250 & 1,600 & 1,020 & 410 & 170 & 3,450 \\
\hline & & 2.0 & 2.0 & 2.0 & 2.0 & 2.0 & 2.0 \\
\hline \multicolumn{2}{|c|}{ Commodity or service } & \multicolumn{6}{|c|}{ Average weekly household expenditure (f) } \\
\hline 1 & Food \& non-alcoholic drinks & 43.20 & 48.10 & 51.80 & 63.10 & 62.00 & 51.30 \\
\hline 2 & Alcoholic drinks, tobacco \& narcotics & 7.60 & 8.10 & 8.60 & 12.50 & 16.30 & 9.10 \\
\hline 3 & Clothing \& footwear & 7.40 & 9.80 & 15.60 & 23.60 & 26.70 & 13.80 \\
\hline 4 & Housing(net) ${ }^{2}$, fuel \& power & 30.30 & 36.90 & 39.90 & 46.30 & 58.50 & 39.50 \\
\hline 5 & Household goods \& services & 22.40 & 19.90 & 31.20 & 47.70 & 53.20 & 28.20 \\
\hline 6 & Health & 4.60 & 5.30 & 9.60 & 11.70 & 12.80 & 7.60 \\
\hline 7 & Transport & 17.30 & 36.40 & 50.30 & 64.90 & 102.90 & 45.60 \\
\hline 8 & Communication & 7.80 & 7.00 & 8.70 & 10.40 & 11.60 & 8.20 \\
\hline 9 & Recreation \& culture & 30.80 & 42.50 & 84.70 & 109.20 & 142.70 & 66.80 \\
\hline 10 & Education & - & {$[0.20]$} & {$[0.20]$} & [3.40] & {$[2.40]$} & 0.70 \\
\hline 11 & Restaurants \& hotels & 10.30 & 17.90 & 28.70 & 52.30 & 61.90 & 26.60 \\
\hline 12 & Miscellaneous goods \& services & 18.80 & 20.70 & 30.20 & 55.80 & 89.50 & 30.70 \\
\hline $1-12$ & All expenditure groups & 200.40 & 252.90 & 359.60 & 500.80 & 640.50 & 328.00 \\
\hline 13 & Other expenditure items & 22.20 & 31.60 & 45.10 & 92.20 & 97.70 & 44.90 \\
\hline \multicolumn{8}{|c|}{ Average weekly expenditure per person (f) } \\
\hline \multicolumn{2}{|c|}{ Total expenditure } & 111.30 & 142.30 & 202.40 & 296.50 & 369.10 & 186.50 \\
\hline
\end{tabular}

Note: The commodity and service categories are not comparable to those in publications before 2001-02. Please see page xiii for symbols and conventions used in this report. This table is based on a three year average.

1 Lower boundary of 2008 gross income quintile groups ( $f$ per week).

2 Excluding mortgage interest payments, council tax and Northern Ireland rates. 


\section{Table A34}

\section{Household expenditure by tenure, 2008}

based on weighted data and including children's expenditure

\begin{tabular}{|c|c|c|c|c|c|c|c|}
\hline & & & Owner & & & ocial rented $f$ & \\
\hline & & $\begin{array}{l}\text { Owned } \\
\text { outright }\end{array}$ & $\begin{array}{r}\text { Buying } \\
\text { with a } \\
\text { mortgage }\end{array}$ & All & Council $^{2}$ & $\begin{array}{r}\text { Registered } \\
\text { Social } \\
\text { Landlord }^{3}\end{array}$ & All \\
\hline Weigl & hted number of households (thousands) & 8,010 & 9,900 & 17,910 & 2,720 & 2,010 & 4,730 \\
\hline Total & number of households in sample & 1,950 & 2,230 & 4,180 & 600 & 440 & 1,040 \\
\hline Total & number of persons in sample & 3,780 & 6,360 & 10,130 & 1,290 & 970 & 2,260 \\
\hline Total & number of adults in sample & 3,510 & 4,480 & 7,990 & 900 & 670 & 1,570 \\
\hline Weigl & hted average number of persons per household & 1.9 & 2.8 & 2.4 & 2.1 & 2.3 & 2.2 \\
\hline Comn & nodity or service & & Average & ekly hous & old expen & diture $(f)$ & \\
\hline 1 & Food \& non-alcoholic drinks & 50.50 & 59.20 & 55.30 & 36.10 & 40.40 & 38.00 \\
\hline 2 & Alcoholic drinks, tobacco \& narcotics & 8.90 & 12.70 & 11.00 & 10.80 & 10.00 & 10.50 \\
\hline 3 & Clothing \& footwear & 17.60 & 28.70 & 23.70 & 11.40 & 18.30 & 14.30 \\
\hline 4 & Housing(net) ${ }^{6}$, fuel \& power & 36.90 & 40.40 & 38.80 & 51.00 & 63.20 & 56.20 \\
\hline 5 & Household goods \& services & 32.20 & 38.70 & 35.80 & 14.10 & 16.30 & 15.00 \\
\hline 6 & Health & 6.00 & 6.40 & 6.20 & 2.10 & 3.10 & 2.50 \\
\hline 7 & Transport & 54.60 & 93.20 & 76.00 & 20.70 & 30.80 & 25.00 \\
\hline 8 & Communication & 9.60 & 15.10 & 12.60 & 8.40 & 10.00 & 9.10 \\
\hline 9 & Recreation \& culture & 69.10 & 73.60 & 71.60 & 28.90 & 31.40 & 30.00 \\
\hline 10 & Education & 4.30 & 8.60 & 6.70 & 1.00 & 1.60 & 1.20 \\
\hline 11 & Restaurants \& hotels & 33.20 & 52.80 & 44.00 & 16.90 & 19.00 & 17.80 \\
\hline 12 & Miscellaneous goods \& services & 32.30 & 49.70 & 41.90 & 12.90 & 16.30 & 14.40 \\
\hline $1-12$ & All expenditure groups & 355.20 & 479.10 & 423.70 & 214.30 & 260.50 & 234.00 \\
\hline 13 & Other expenditure items & 46.10 & 157.80 & 107.90 & 13.70 & 23.50 & 17.90 \\
\hline Total & I expenditure & 401.20 & 637.00 & 531.60 & 228.00 & 284.00 & 251.90 \\
\hline $\begin{array}{l}\text { Aver } \\
\text { Total }\end{array}$ & $\begin{array}{l}\text { age weekly expendi } \\
\text { l expenditure }\end{array}$ & 210.20 & 225.90 & 220.30 & 108.30 & 125.50 & 115.90 \\
\hline
\end{tabular}

Note: The commodity and service categories are not comparable to those in publications before 2001-02. Please see page xiii for symbols and conventions used in this report.

1 Including shared owners (who own part of the equity and pay mortgage, part rent).

2 "Council" includes local authorities, New Towns and Scottish Homes, but see note 3 below.

3 Formerly Housing Associations. 


\section{Table A34}

Household expenditure by tenure, 2008 (cont.)

based on weighted data and including children's expenditure

\begin{tabular}{|c|c|c|c|c|c|c|}
\hline & & & & te rented ${ }^{4}$ & & All \\
\hline & & $\begin{array}{l}\text { Rent } \\
\text { free }\end{array}$ & $\begin{array}{l}\text { Rent paid } \\
\text { unfurn- } \\
\text { ished }^{5}\end{array}$ & $\begin{array}{r}\text { Rent } \\
\text { paid, } \\
\text { furnished }\end{array}$ & All & \\
\hline Weig & hted number of households (thousands) & 340 & 2,130 & 580 & 3,040 & 25,690 \\
\hline Total & number of households in sample & 70 & 450 & 110 & 630 & 5,850 \\
\hline Total & number of persons in sample & 120 & 1,050 & 270 & 1,440 & 13,830 \\
\hline Total & number of adults in sample & 110 & 750 & 220 & 1,080 & 10,640 \\
\hline Weig & hted average number of persons per household & 1.7 & 2.4 & 2.5 & 2.3 & 2.4 \\
\hline Com & nodity or service & & Average wee & ly househol & xpenditur & \\
\hline 1 & Food \& non-alcoholic drinks & 38.10 & 43.50 & 44.90 & 43.20 & 50.70 \\
\hline 2 & Alcoholic drinks, tobacco \& narcotics & 7.40 & 10.80 & 9.20 & 10.10 & 10.80 \\
\hline 3 & Clothing \& footwear & 13.20 & 19.50 & 27.40 & 20.30 & 21.60 \\
\hline 4 & Housing(net) ${ }^{6}$, fuel \& power & 25.50 & 140.10 & 162.40 & 131.70 & 53.00 \\
\hline 5 & Household goods \& services & 21.90 & 22.50 & 11.30 & 20.30 & 30.10 \\
\hline 6 & Health & 2.40 & 3.10 & 1.50 & 2.70 & 5.10 \\
\hline 7 & Transport & 47.20 & 48.60 & 51.80 & 49.10 & 63.40 \\
\hline 8 & Communication & 8.10 & 13.10 & 13.40 & 12.60 & 12.00 \\
\hline 9 & Recreation \& culture & 34.70 & 40.50 & 36.50 & 39.10 & 60.10 \\
\hline 10 & Education & [13.10] & 10.80 & [10.60] & 11.00 & 6.20 \\
\hline 11 & Restaurants \& hotels & 20.80 & 33.20 & 31.90 & 31.60 & 37.70 \\
\hline 12 & Miscellaneous goods \& services & 23.20 & 34.40 & 26.90 & 31.70 & 35.60 \\
\hline 1-12 & All expenditure groups & 255.50 & 420.10 & 427.70 & 403.40 & 386.30 \\
\hline 13 & Other expenditure items & 32.20 & 58.60 & 38.40 & 51.80 & 84.60 \\
\hline Tota & expenditure & 287.70 & 478.70 & 466.00 & 455.20 & 471.00 \\
\hline $\begin{array}{l}\text { Aver } \\
\text { Tota }\end{array}$ & $\begin{array}{l}\text { age weekly expenditure per person (f) } \\
\text { expenditure }\end{array}$ & 165.10 & 203.00 & 185.70 & 196.30 & 199.80 \\
\hline
\end{tabular}

Note: The commodity and service categories are not comparable to those in publications before 2001-02.

Please see page xiii for symbols and conventions used in this report.

4 All tenants whose accommodation goes with the job of someone in the household are allocated to "rented privately", even if the landlord is a local authority, housing association or Housing Action Trust, or if the accommodation is rent free. Squatters are also included in this category.

5 "Unfurnished" includes the answers: "partly furnished".

6 Excluding mortgage interest payments, council tax and Northern Ireland rates. 


\section{Table A35}

\section{Household expenditure by UK countries and} Government Office Regions, 2006-2008

based on weighted data and including children's expenditure

\begin{tabular}{|c|c|c|c|c|c|c|c|c|}
\hline & & $\begin{array}{r}\text { North } \\
\text { East }\end{array}$ & $\begin{array}{c}\text { North } \\
\text { West }\end{array}$ & $\begin{array}{r}\text { Yorks } \\
\& \text { the } \\
\text { Humber }\end{array}$ & $\begin{array}{r}\text { East } \\
\text { Midlands }\end{array}$ & $\begin{array}{r}\text { West } \\
\text { Midlands }\end{array}$ & East & London \\
\hline \multicolumn{2}{|r|}{ Average weighted number of households (thousands) } & 1,130 & 2,930 & 2,150 & 1,900 & 2,150 & 2,310 & 3,040 \\
\hline \multicolumn{2}{|r|}{ Total number of households in sample (over 3 years) } & 770 & 1,890 & 1,570 & 1,360 & 1,510 & 1,670 & 1,540 \\
\hline \multicolumn{2}{|c|}{ Total number of persons in sample (over 3 years) } & 1,820 & 4,490 & 3,700 & 3,240 & 3,730 & 3,950 & 3,700 \\
\hline \multicolumn{2}{|r|}{ Total number of adults in sample (over 3 years) } & 1,430 & 3,390 & 2,790 & 2,460 & 2,820 & 3,020 & 2,770 \\
\hline \multicolumn{2}{|c|}{ Weighted average number of persons per household } & 2.4 & 2.4 & 2.3 & 2.3 & 2.5 & 2.3 & 2.5 \\
\hline \multicolumn{3}{|c|}{ Commodity or service } & \multicolumn{6}{|c|}{ Average weekly household expenditure (f) } \\
\hline 1 & Food \& non-alcoholic drinks & 43.10 & 46.20 & 45.30 & 46.80 & 48.60 & 49.00 & 50.20 \\
\hline 2 & Alcoholic drinks, tobacco \& narcotics & 10.50 & 12.20 & 10.50 & 10.40 & 11.80 & 10.30 & 10.00 \\
\hline 3 & Clothing \& footwear & 21.60 & 21.50 & 20.10 & 19.40 & 23.80 & 21.90 & 26.10 \\
\hline 4 & Housing(net) ${ }^{1}$, fuel \& power & 42.40 & 42.70 & 46.20 & 43.10 & 44.70 & 53.10 & 73.90 \\
\hline 6 & Health & 3.60 & 5.90 & 4.50 & 6.30 & 4.70 & 6.10 & 7.00 \\
\hline 7 & Transport & 49.70 & 52.60 & 54.60 & 60.60 & 59.60 & 68.70 & 63.50 \\
\hline 8 & Communication & 10.30 & 11.20 & 10.60 & 11.40 & 11.50 & 12.70 & 14.50 \\
\hline 9 & Recreation \& culture & 50.60 & 56.70 & 55.50 & 56.60 & 58.30 & 63.80 & 54.30 \\
\hline 10 & Education & 4.60 & 4.00 & 4.50 & 4.10 & 4.60 & 6.00 & 14.80 \\
\hline 11 & Restaurants \& hotels & 33.80 & 35.70 & 37.20 & 35.10 & 36.10 & 37.60 & 45.00 \\
\hline 12 & Miscellaneous goods $\&$ services & 28.40 & 34.10 & 30.30 & 32.10 & 34.60 & 40.60 & 39.40 \\
\hline $1-12$ & All expenditure groups & 327.90 & 348.70 & 349.80 & 353.20 & 367.30 & 404.20 & 430.60 \\
\hline 13 & Other expenditure items & 58.20 & 72.50 & 64.80 & 69.00 & 69.80 & 89.10 & 114.10 \\
\hline \multicolumn{2}{|c|}{ Total expenditure } & 386.10 & 421.20 & 414.60 & 422.30 & 437.10 & 493.40 & $\mathbf{5 4 4 . 7 0}$ \\
\hline
\end{tabular}

Note: The commodity and service categories are not comparable to those in publications before 2001-02.

Please see page xiii for symbols and conventions used in this report.

This table is based on a three year average.

1 Excluding mortgage interest payments, council tax and Northern Ireland rates. 


\section{Table A35}

\section{Household expenditure by UK countries and} Government Office Regions, 2006-2008 (cont.)

based on weighted data and including children's expenditure

\begin{tabular}{|c|c|c|c|c|c|c|c|}
\hline & $\begin{array}{l}\text { South } \\
\text { East }\end{array}$ & $\begin{array}{l}\text { South } \\
\text { West }\end{array}$ & England & Wales & Scotland & $\begin{array}{l}\text { Northern } \\
\text { Ireland }\end{array}$ & $\begin{array}{r}\text { United } \\
\text { Kingdom }\end{array}$ \\
\hline Average weighted number of households (thousands) & 3,280 & 2,350 & 21,240 & 1,270 & 2,320 & 650 & 25,490 \\
\hline Total number of households in sample (over 3 years) & 2,530 & 1,580 & 14,440 & 860 & 1,580 & 1,760 & 18,630 \\
\hline Total number of persons in sample (over 3 years) & 6,080 & 3,540 & 34,260 & 1,970 & 3,530 & 4,570 & 44,330 \\
\hline Total number of adults in sample (over 3 years) & 4,660 & 2,850 & 26,190 & 1,550 & 2,790 & 3,340 & 33,860 \\
\hline Weighted average number of persons per household & 2.3 & 2.3 & 2.4 & 2.3 & 2.2 & 2.7 & 2.4 \\
\hline
\end{tabular}

Commodity or service

Average weekly household expenditure (f)

\begin{tabular}{|c|c|c|c|c|c|c|c|c|}
\hline 1 & Food \& non-alcoholic drinks & 51.70 & 49.80 & 48.40 & 47.70 & 46.80 & 55.40 & 48.40 \\
\hline 2 & Alcoholic drinks, tobacco \& narcotics & 10.70 & 10.30 & 10.80 & 11.60 & 12.30 & 13.80 & 11.00 \\
\hline 3 & Clothing \& footwear & 21.10 & 19.90 & 21.90 & 19.40 & 23.30 & 34.50 & 22.20 \\
\hline 5 & Household goods \& services & 32.90 & 31.50 & 30.50 & 25.70 & 30.00 & 32.80 & 30.30 \\
\hline 6 & Health & 6.40 & 6.00 & 5.80 & 3.50 & 4.50 & 4.40 & 5.60 \\
\hline 7 & Transport & 76.40 & 65.60 & 62.50 & 56.20 & 59.90 & 62.80 & 62.00 \\
\hline 8 & Communication & 11.90 & 11.70 & 11.90 & 10.60 & 11.00 & 14.60 & 11.80 \\
\hline 9 & Recreation \& culture & 61.70 & 63.60 & 58.30 & 58.20 & 59.20 & 55.90 & 58.30 \\
\hline 10 & Education & 7.80 & 8.90 & 7.10 & 5.00 & 4.30 & 4.60 & 6.70 \\
\hline 11 & Restaurants \& hotels & 39.30 & 36.70 & 37.90 & 30.90 & 35.40 & 44.30 & 37.50 \\
\hline 12 & Miscellaneous goods \& services & 42.00 & 35.20 & 36.10 & 29.70 & 32.50 & 39.20 & 35.50 \\
\hline 13 & Other expenditure items & 94.80 & 76.70 & 82.00 & 60.40 & 71.10 & 72.40 & 79.70 \\
\hline Total & expenditure & 512.30 & 469.20 & 465.20 & 406.70 & 432.80 & 479.70 & 459.70 \\
\hline \multicolumn{9}{|c|}{ Average weekly expenditure per person (f) } \\
\hline \multicolumn{2}{|c|}{ Total expenditure } & 220.10 & 205.40 & 197.00 & 175.90 & 197.90 & 180.50 & 195.60 \\
\hline
\end{tabular}

Note: The commodity and service categories are not comparable to those in publications before 2001-02.

Please see page xiii for symbols and conventions used in this report.

This table is based on a three year average.

1 Excluding mortgage interest payments, council tax and Northern Ireland rates. 


\section{Table A36}

\section{Household expenditure as a percentage of total expenditure} by UK countries and Government Office Regions, 2006-2008

based on weighted data and including children's expenditure

\begin{tabular}{|c|c|c|c|c|c|c|c|c|}
\hline & & $\begin{array}{r}\text { North } \\
\text { East }\end{array}$ & $\begin{array}{l}\text { North } \\
\text { West }\end{array}$ & $\begin{array}{r}\text { Yorks } \\
\text { \& the } \\
\text { Humber }\end{array}$ & $\begin{array}{r}\text { East } \\
\text { Midlands }\end{array}$ & $\begin{array}{r}\text { West } \\
\text { Midlands }\end{array}$ & East & London \\
\hline \multicolumn{2}{|c|}{ Average weighted number of households (thousands) } & 1,130 & 2,930 & 2,150 & 1,900 & 2,150 & 2,310 & 3,040 \\
\hline \multicolumn{2}{|r|}{ Total number of households in sample (over 3 years) } & 770 & 1,890 & 1,570 & 1,360 & 1,510 & 1,670 & 1,540 \\
\hline \multicolumn{2}{|c|}{ Total number of persons in sample (over 3 years) } & 1,820 & 4,490 & 3,700 & 3,240 & 3,730 & 3,950 & 3,700 \\
\hline \multirow{2}{*}{\multicolumn{2}{|c|}{$\begin{array}{l}\text { Total number of adults in sample (over } 3 \text { years) } \\
\text { Weighted average number of persons per household }\end{array}$}} & 1,430 & 3,390 & 2,790 & 2,460 & 2,820 & 3,020 & 2,770 \\
\hline & & 2.4 & 2.4 & 2.3 & 2.3 & 2.5 & 2.3 & 2.5 \\
\hline \multicolumn{2}{|c|}{ Commodity or service } & \multicolumn{7}{|c|}{ Percentage of total expenditure } \\
\hline 1 & Food \& non-alcoholic drinks & 11 & 11 & 11 & 11 & 11 & 10 & 9 \\
\hline 2 & Alcoholic drinks, tobacco \& narcotics & 3 & 3 & 3 & 2 & 3 & 2 & 2 \\
\hline 3 & Clothing \& footwear & 6 & 5 & 5 & 5 & 5 & 4 & 5 \\
\hline 4 & Housing(net)' ${ }^{1}$ fuel \& power & 11 & 10 & 11 & 10 & 10 & 11 & 14 \\
\hline 5 & Household goods \& services & 8 & 6 & 7 & 7 & 7 & 7 & 6 \\
\hline 6 & Health & 1 & 1 & 1 & 1 & 1 & 1 & 1 \\
\hline 7 & Transport & 13 & 12 & 13 & 14 & 14 & 14 & 12 \\
\hline 8 & Communication & 3 & 3 & 3 & 3 & 3 & 3 & 3 \\
\hline 9 & Recreation \& culture & 13 & 13 & 13 & 13 & 13 & 13 & 10 \\
\hline 10 & Education & 1 & 1 & 1 & 1 & 1 & 1 & 3 \\
\hline 11 & Restaurants \& hotels & 9 & 8 & 9 & 8 & 8 & 8 & 8 \\
\hline 12 & Miscellaneous goods \& services & 7 & 8 & 7 & 8 & 8 & 8 & 7 \\
\hline $1-12$ & All expenditure groups & 85 & 83 & 84 & 84 & 84 & 82 & 79 \\
\hline 13 & Other expenditure items & 15 & 17 & 16 & 16 & 16 & 18 & 21 \\
\hline Total & expenditure & 100 & 100 & 100 & 100 & 100 & 100 & 100 \\
\hline
\end{tabular}

Note: The commodity and service categories are not comparable to those in publications before 2001-02.

Please see page xiii for symbols and conventions used in this report.

This table is based on a three year average.

1 Excluding mortgage interest payments, council tax and Northern Ireland rates. 


\section{Table A36}

\section{Household expenditure as a percentage of total expenditure} by UK countries and Government Office Regions, 2006-2008 (cont.)

based on weighted data and including children's expenditure

\begin{tabular}{|c|c|c|c|c|c|c|c|}
\hline & $\begin{array}{r}\text { South } \\
\text { East }\end{array}$ & $\begin{array}{l}\text { South } \\
\text { West }\end{array}$ & England & Wales & Scotland & $\begin{array}{l}\text { Northern } \\
\text { Ireland }\end{array}$ & $\begin{array}{r}\text { United } \\
\text { Kingdom }\end{array}$ \\
\hline Average weighted number of households (thousands) & 3,280 & 2,350 & 21,240 & 1,270 & 2,320 & 650 & 25,490 \\
\hline Total number of households in sample (over 3 years) & 2,530 & 1,580 & 14,440 & 860 & 1,580 & 1,760 & 18,630 \\
\hline Total number of persons in sample (over 3 years) & 6,080 & 3,540 & 34,260 & 1,970 & 3,530 & 4,570 & 44,330 \\
\hline Total number of adults in sample (over 3 years) & 4,660 & 2,850 & 26,190 & 1,550 & 2,790 & 3,340 & 33,860 \\
\hline Weighted average number of persons per household & 2.3 & 2.3 & 2.4 & 2.3 & 2.2 & 2.7 & 2.4 \\
\hline
\end{tabular}

Commodity or service

Percentage of total expenditure

\begin{tabular}{|c|c|c|c|c|c|c|c|c|}
\hline 1 & Food \& non-alcoholic drinks & 10 & 11 & 10 & 12 & 11 & 12 & 11 \\
\hline 2 & Alcoholic drinks, tobacco \& narcotics & 2 & 2 & 2 & 3 & 3 & 3 & 2 \\
\hline 3 & Clothing \& footwear & 4 & 4 & 5 & 5 & 5 & 7 & 5 \\
\hline 4 & Housing(net)', fuel \& power & 11 & 11 & 11 & 12 & 10 & 9 & 11 \\
\hline 5 & Household goods \& services & 6 & 7 & 7 & 6 & 7 & 7 & 7 \\
\hline 6 & Health & 1 & 1 & 1 & 1 & 1 & 1 & 1 \\
\hline 7 & Transport & 15 & 14 & 13 & 14 & 14 & 13 & 13 \\
\hline 8 & Communication & 2 & 2 & 3 & 3 & 3 & 3 & 3 \\
\hline 9 & Recreation \& culture & 12 & 14 & 13 & 14 & 14 & 12 & 13 \\
\hline 10 & Education & 2 & 2 & 2 & 1 & 1 & 1 & 1 \\
\hline 11 & Restaurants \& hotels & 8 & 8 & 8 & 8 & 8 & 9 & 8 \\
\hline 12 & Miscellaneous goods \& services & 8 & 7 & 8 & 7 & 8 & 8 & 8 \\
\hline $1-12$ & All expenditure groups & 81 & 84 & 82 & 85 & 84 & 85 & 83 \\
\hline 13 & Other expenditure items & 19 & 16 & 18 & 15 & 16 & 15 & 17 \\
\hline
\end{tabular}

Note: The commodity and service categories are not comparable to those in publications before 2001-02.

Please see page xiii for symbols and conventions used in this report.

This table is based on a three year average.

1 Excluding mortgage interest payments, council tax and Northern Ireland rates. 


\section{Table A37}

\section{Detailed household expenditure by UK countries and Government Office Regions, 2006-2008}

based on weighted data and including children's expenditure

\begin{tabular}{|c|c|c|c|c|c|c|c|c|c|}
\hline & & & $\begin{array}{l}\text { North } \\
\text { East }\end{array}$ & $\begin{array}{c}\text { North } \\
\text { West }\end{array}$ & $\begin{array}{r}\text { Yorkshire } \\
\& \text { the } \\
\text { Humber }\end{array}$ & $\begin{array}{r}\text { East } \\
\text { Midlands }\end{array}$ & $\begin{array}{r}\text { West } \\
\text { Midlands }\end{array}$ & East & London \\
\hline \multicolumn{3}{|c|}{ Average weighted number of households (thousands) } & 1,130 & 2,930 & 2,150 & 1,900 & 2,150 & 2,310 & 3,040 \\
\hline \multicolumn{3}{|c|}{ Total number of households in sample (over 3 years) } & 770 & 1,890 & 1,570 & 1,360 & 1,510 & 1,670 & 1,540 \\
\hline \multicolumn{3}{|c|}{ Total number of persons in sample (over 3 years) } & 1,820 & 4,490 & 3,700 & 3,240 & 3,730 & 3,950 & 3,700 \\
\hline \multirow{2}{*}{\multicolumn{3}{|c|}{$\begin{array}{l}\text { Total number of adults in sample (over } 3 \text { years) } \\
\text { Weighted average number of persons per household }\end{array}$}} & 1,430 & 3,390 & 2,790 & 2,460 & 2,820 & 3,020 & 2,770 \\
\hline & & & 2.4 & 2.4 & 2.3 & 2.3 & 2.5 & 2.3 & 2.5 \\
\hline \multicolumn{5}{|c|}{ Commodity or service } & \multicolumn{5}{|c|}{ Average weekly household expenditure $(f)$} \\
\hline \multirow[t]{36}{*}{ oo } & \multicolumn{2}{|c|}{ ood \& non-alcoholic drinks } & 43.10 & 46.20 & 45.30 & 46.80 & 48.60 & 49.00 & 50.20 \\
\hline & Food & & 39.60 & 42.20 & 41.80 & 43.00 & 44.60 & 45.00 & 45.70 \\
\hline & 1.1 .1 & Bread, rice and cereals & 4.30 & 4.40 & 4.30 & 4.50 & 4.70 & 4.40 & 4.60 \\
\hline & 1.1 .2 & Pasta products & 0.30 & 0.30 & 0.30 & 0.30 & 0.30 & 0.30 & 0.40 \\
\hline & 1.1 .3 & Buns, cakes, biscuits etc. & 2.80 & 2.80 & 2.80 & 3.00 & 2.90 & 3.10 & 2.60 \\
\hline & 1.1 .5 & Beef (fresh, chilled or frozen) & 1.50 & 1.60 & 1.50 & 1.40 & 1.70 & 1.60 & 1.40 \\
\hline & 1.1.6 & Pork (fresh, chilled or frozen) & 0.40 & 0.50 & 0.60 & 0.60 & 0.70 & 0.70 & 0.60 \\
\hline & 1.1 .7 & Lamb (fresh, chilled or frozen) & 0.30 & 0.70 & 0.50 & 0.50 & 0.90 & 0.70 & 0.90 \\
\hline & 1.1 .8 & Poultry (fresh, chilled or frozen) & 1.50 & 1.70 & 1.60 & 1.60 & 1.90 & 1.80 & 1.90 \\
\hline & 1.1 .9 & Bacon and ham & 0.90 & 1.00 & 1.00 & 0.90 & 0.90 & 0.80 & 0.60 \\
\hline & 1.1 .10 & Other meat and meat preparations & 5.10 & 5.20 & 5.00 & 4.80 & 5.00 & 5.10 & 4.20 \\
\hline & 1.1.11 & Fish and fish products & 1.90 & 2.20 & 2.10 & 2.00 & 2.10 & 2.30 & 2.90 \\
\hline & 1.1.12 & Milk & 2.50 & 2.50 & 2.50 & 2.60 & 2.50 & 2.30 & 2.20 \\
\hline & 1.1.13 & Cheese and curd & 1.30 & 1.40 & 1.40 & 1.60 & 1.60 & 1.70 & 1.60 \\
\hline & 1.1.14 & Eggs & 0.50 & 0.50 & 0.50 & 0.50 & 0.50 & 0.50 & 0.60 \\
\hline & 1.1.15 & Other milk products & 1.50 & 1.60 & 1.60 & 1.70 & 1.60 & 1.90 & 1.70 \\
\hline & 1.1.16 & Butter & 0.30 & 0.30 & 0.30 & 0.30 & 0.30 & 0.30 & 0.30 \\
\hline & \multirow[t]{2}{*}{ 1.1.17 } & Margarine, other vegetable & & & & & & & \\
\hline & & fats and peanut butter & 0.40 & 0.40 & 0.40 & 0.50 & 0.50 & 0.50 & 0.40 \\
\hline & 1.1.18 & Cooking oils and fats & 0.20 & 0.20 & 0.20 & 0.30 & 0.20 & 0.20 & 0.40 \\
\hline & 1.1.19 & Fresh fruit & 2.20 & 2.40 & 2.60 & 2.70 & 2.70 & 3.00 & 3.70 \\
\hline & \multirow[t]{2}{*}{1.1 .22} & Preserved fruit and fruit & & & & & & & \\
\hline & & based products & 0.10 & 0.10 & 0.10 & 0.10 & 0.10 & 0.10 & 0.10 \\
\hline & 1.1 .23 & Fresh vegetables & 2.90 & 3.10 & 3.20 & 3.60 & 3.60 & 3.90 & 4.60 \\
\hline & 1.1.24 & Dried vegetables & 0.00 & 0.00 & 0.00 & 0.00 & 0.00 & 0.00 & 0.10 \\
\hline & \multirow[t]{2}{*}{1.1 .25} & Other preserved or processed & & & & & & & \\
\hline & & vegetables & 1.00 & 1.00 & 1.00 & 1.10 & 1.00 & 1.10 & 1.30 \\
\hline & 1.1 .26 & Potatoes & 0.80 & 0.80 & 0.80 & 0.80 & 0.90 & 0.80 & 0.70 \\
\hline & \multirow[t]{2}{*}{1.1 .27} & Other tubers and products of & & & & & & & \\
\hline & & tuber vegetables & 1.20 & 1.20 & 1.20 & 1.20 & 1.30 & 1.20 & 0.90 \\
\hline & 1.1 .28 & Sugar and sugar products & 0.20 & 0.30 & 0.30 & 0.30 & 0.30 & 0.30 & 0.30 \\
\hline & 1.1 .29 & Jams, marmalades & 0.20 & 0.30 & 0.30 & 0.30 & 0.30 & 0.30 & 0.30 \\
\hline & 1.1 .30 & Chocolate & 1.40 & 1.40 & 1.40 & 1.50 & 1.50 & 1.40 & 1.30 \\
\hline & 1.1 .31 & Confectionery products & 0.60 & 0.60 & 0.60 & 0.60 & 0.60 & 0.50 & 0.50 \\
\hline & 1.1 .32 & Edible ices and ice cream & 0.40 & 0.50 & 0.40 & 0.50 & 0.40 & 0.50 & 0.50 \\
\hline & 1.1 .33 & Other food products & 1.80 & 2.10 & 2.00 & 2.00 & 2.10 & 2.00 & 2.50 \\
\hline \multirow[t]{9}{*}{1.2} & \multicolumn{2}{|c|}{ Non-alcoholic drinks } & 3.50 & 3.90 & 3.60 & 3.80 & 4.00 & 4.00 & 4.40 \\
\hline & 1.2 .1 & Coffee & 0.50 & 0.50 & 0.50 & 0.50 & 0.50 & 0.60 & 0.40 \\
\hline & 1.2 .2 & Tea & 0.40 & 0.40 & 0.40 & 0.50 & 0.50 & 0.40 & 0.40 \\
\hline & 1.2 .3 & Cocoa and powdered chocolate & 0.10 & 0.10 & 0.10 & 0.10 & 0.10 & 0.10 & 0.10 \\
\hline & \multirow[t]{2}{*}{1.2 .4} & Fruit and vegetable juices & & & & & & & \\
\hline & & (inc. fruit squash) & 0.90 & 1.10 & 1.00 & 1.10 & 1.10 & 1.20 & 1.40 \\
\hline & 1.2 .5 & Mineral or spring waters & 0.20 & 0.20 & 0.20 & 0.20 & 0.20 & 0.20 & 0.50 \\
\hline & \multirow[t]{2}{*}{1.2 .6} & Soft drinks (inc. fizzy and ready & & & & & & & \\
\hline & & to drink fruit drinks) & 1.50 & 1.60 & 1.40 & 1.50 & 1.60 & 1.50 & 1.60 \\
\hline
\end{tabular}

Note: The commodity and service categories are not comparable to those in publications before $2001-02$.

The numbering system is sequential, it does not use actual COICOP codes.

Please see page xiii for symbols and conventions used in this report.

This table is based on a three year average. 


\section{Table A37}

\section{Detailed household expenditure by UK countries and Government Office Regions, 2006-2008 (cont.)}

based on weighted data and including children's expenditure

\begin{tabular}{|c|c|c|c|c|c|c|c|}
\hline & $\begin{array}{r}\text { South } \\
\text { East }\end{array}$ & $\begin{array}{r}\text { South } \\
\text { West }\end{array}$ & England & Wales & Scotland & $\begin{array}{l}\text { Northern } \\
\text { Ireland }\end{array}$ & $\begin{array}{r}\text { United } \\
\text { Kingdom }\end{array}$ \\
\hline Average weighted number of households (thousands) & 3,280 & 2,350 & 21,240 & 1,270 & 2,320 & 650 & 25,490 \\
\hline Total number of households in sample (over 3 years) & 2,530 & 1,580 & 14,440 & 860 & 1,580 & 1,760 & 18,630 \\
\hline Total number of persons in sample (over 3 years) & 6,080 & 3,540 & 34,260 & 1,970 & 3,530 & 4,570 & 44,330 \\
\hline Total number of adults in sample (over 3 years) & 4,660 & 2,850 & 26,190 & 1,550 & 2,790 & 3,340 & 33,860 \\
\hline Weighted average number of persons per household & 2.3 & 2.3 & 2.4 & 2.3 & 2.2 & 2.7 & 2.4 \\
\hline
\end{tabular}

Average weekly household expenditure (f)

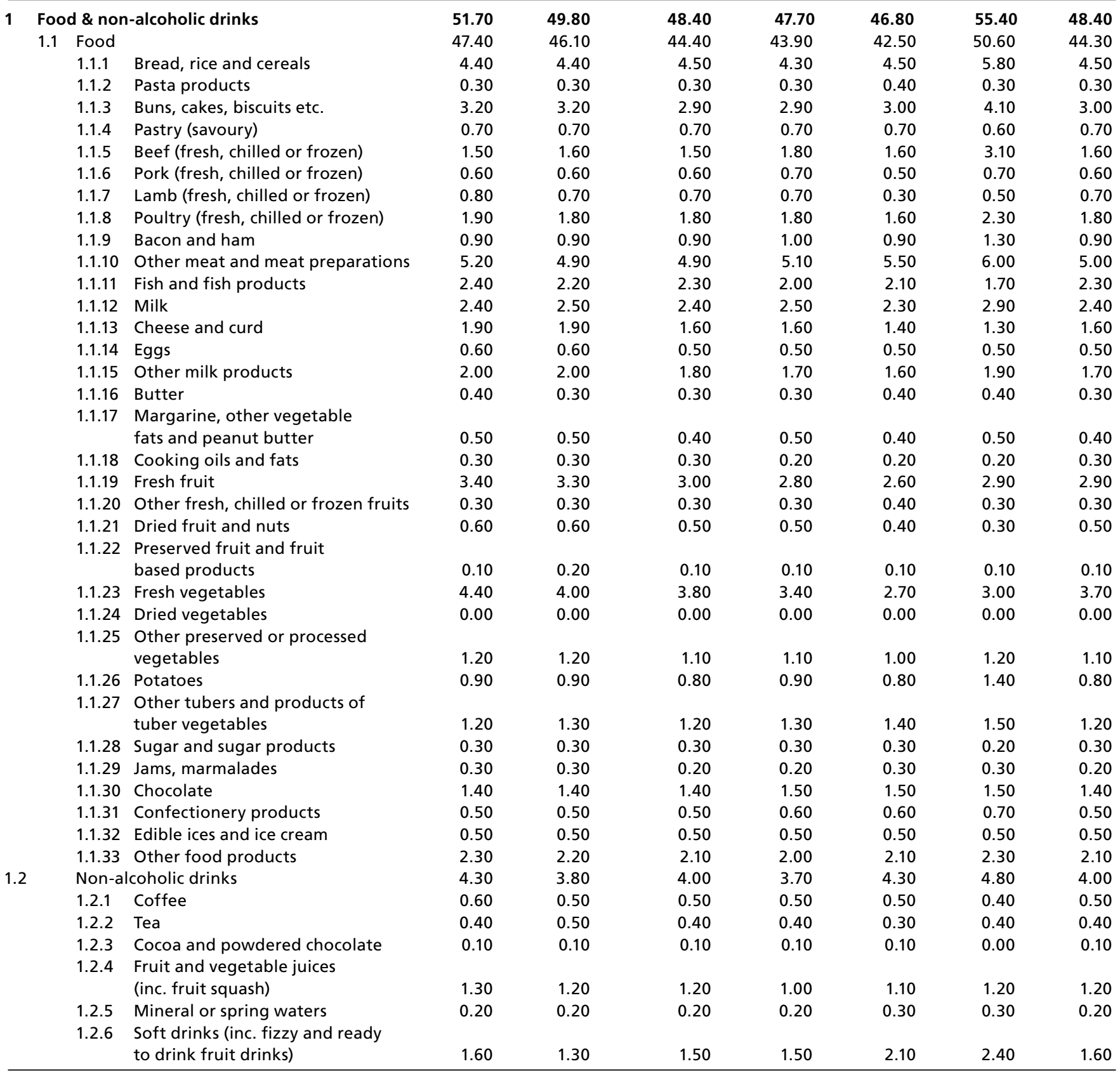

Note: The commodity and service categories are not comparable to those in publications before 2001-02.

The numbering system is sequential, it does not use actual COICOP codes.

Please see page xiii for symbols and conventions used in this report.

This table is based on a three year average. 


\section{Table A37}

\section{Detailed household expenditure by UK countries and Government Office Regions, 2006-2008 (cont.)}

based on weighted data and including children's expenditure

\begin{tabular}{|c|c|c|c|c|c|c|c|c|c|c|}
\hline & & & & $\begin{array}{r}\text { North } \\
\text { East }\end{array}$ & $\begin{array}{l}\text { North } \\
\text { West }\end{array}$ & $\begin{array}{c}\text { Yorkshire } \\
\text { \& the } \\
\text { Humber }\end{array}$ & $\begin{array}{r}\text { East } \\
\text { Midlands }\end{array}$ & $\begin{array}{r}\text { West } \\
\text { Midlands }\end{array}$ & East & London \\
\hline \multicolumn{6}{|c|}{ Commodity or service } & \multicolumn{5}{|c|}{ Average weekly household expenditure ( $f$ ) } \\
\hline 2 & \multicolumn{3}{|c|}{ Alcoholic drink, tobacco \& narcotics } & 10.50 & 12.20 & 10.50 & 10.40 & 11.80 & 10.30 & 10.00 \\
\hline & \multirow{5}{*}{\multicolumn{2}{|c|}{$\begin{array}{cl}2.1 & \text { Alcoh } \\
& 2.1 .1 \\
& 2.1 .2 \\
& 2.1 .3\end{array}$}} & lic drinks & 6.00 & 7.10 & 6.00 & 5.90 & 6.50 & 6.60 & 6.00 \\
\hline & & & Spirits and liqueurs (brought home) & 1.00 & 1.50 & 0.90 & 1.10 & 1.50 & 1.20 & 1.00 \\
\hline & & & Wines, fortified wines (brought home) & 2.90 & 3.40 & 2.90 & 2.90 & 3.10 & 3.70 & 3.60 \\
\hline & & & Beer, lager, ciders and perry & & & & & & & \\
\hline & & & (brought home) & 2.00 & 2.10 & 2.10 & 1.80 & 1.90 & 1.60 & 1.40 \\
\hline & & 2.1.4 & Alcopops (brought home) & 0.10 & 0.10 & 0.10 & 0.10 & 0.10 & 0.10 & 0.00 \\
\hline & \multirow{4}{*}{2.2} & \multicolumn{2}{|c|}{ Tobacco and narcotics } & 4.50 & 5.10 & 4.50 & 4.50 & 5.30 & 3.70 & 4.00 \\
\hline & & 2.2.1 & Cigarettes & 4.10 & 4.60 & 4.00 & 4.00 & 4.60 & 3.20 & 3.60 \\
\hline & & 2.2 .2 & Cigars, other tobacco & & & & & & & \\
\hline & & & products and narcotics & 0.40 & 0.50 & 0.50 & 0.50 & 0.70 & 0.60 & 0.40 \\
\hline \multirow[t]{15}{*}{3} & \multicolumn{3}{|c|}{ Clothing \& footwear } & 21.60 & 21.50 & 20.10 & 19.40 & 23.80 & 21.90 & 26.10 \\
\hline & \multirow[t]{13}{*}{3.1} & \multicolumn{2}{|c|}{ Clothing } & 17.50 & 17.10 & 16.60 & 15.20 & 19.10 & 17.80 & 20.80 \\
\hline & & 3.1 .1 & Men's outer garments & 4.80 & 3.80 & 3.70 & 4.00 & 5.00 & 4.80 & 5.70 \\
\hline & & 3.1 .2 & Men's under garments & 0.40 & 0.30 & 0.40 & 0.30 & 0.30 & 0.30 & 0.50 \\
\hline & & 3.1 .3 & Women's outer garments & 7.70 & 7.40 & 7.80 & 6.50 & 8.50 & 8.00 & 9.10 \\
\hline & & 3.1.4 & Women's under garments & 1.00 & 1.10 & 1.10 & 1.00 & 1.20 & 1.10 & 1.40 \\
\hline & & 3.1.5 & Boys' outer garments (5-15) & 0.70 & 0.80 & 0.70 & 0.80 & 1.00 & 0.80 & 0.60 \\
\hline & & 3.1 .6 & Girls' outer garments (5-15) & 1.00 & 1.30 & 1.00 & 0.90 & 0.90 & 0.80 & 0.90 \\
\hline & & 3.1.7 & Infants' outer garments (under 5) & 0.80 & 0.80 & 0.60 & 0.60 & 0.80 & 0.50 & 0.60 \\
\hline & & 3.1 .8 & Children's under garments (under 16) & 0.40 & 0.40 & 0.30 & 0.30 & 0.30 & 0.30 & 0.40 \\
\hline & & 3.1 .9 & Accessories & 0.60 & 0.70 & 0.70 & 0.50 & 0.70 & 0.70 & 0.90 \\
\hline & & 3.1 .10 & Haberdashery, clothing materials & & & & & & & \\
\hline & & & and clothing hire & 0.10 & 0.30 & 0.20 & 0.20 & 0.30 & 0.20 & 0.20 \\
\hline & & 3.1.11 & Dry cleaners, laundry and dyeing & {$[0.10]$} & 0.20 & 0.20 & 0.20 & 0.30 & 0.30 & 0.60 \\
\hline & 3.2 & Footh & ear & 4.10 & 4.40 & 3.60 & 4.10 & 4.70 & 4.10 & 5.40 \\
\hline \multirow[t]{14}{*}{4} & \multicolumn{3}{|c|}{ Housing (net) ${ }^{1}$, fuel \& power } & 42.40 & 42.70 & 46.20 & 43.10 & 44.70 & 53.10 & 73.90 \\
\hline & \multirow[t]{6}{*}{4.1} & \multicolumn{2}{|c|}{ Actual rentals for housing } & 26.60 & 24.50 & 25.70 & 23.90 & 26.20 & 26.90 & 62.60 \\
\hline & & 4.1.1 & Gross rent & 26.40 & 24.30 & 25.70 & 23.70 & 26.20 & 26.90 & 62.60 \\
\hline & & 4.1 .2 & less housing benefit, & & & & & & & \\
\hline & & & rebates \& allowances rec'd & 12.40 & 12.00 & 10.60 & 9.70 & 11.70 & 8.50 & 22.20 \\
\hline & & 4.1.3 & Net rent ${ }^{2}$ & 14.10 & 12.30 & 15.10 & 14.00 & 14.50 & 18.40 & 40.40 \\
\hline & & 4.1.4 & Second dwelling rent & {$[0.20]$} & {$[0.30]$} & {$[0.00]$} & {$[0.20]$} & - & {$[0.00]$} & - \\
\hline & \multirow{3}{*}{4.3} & \multirow{2}{*}{\multicolumn{2}{|c|}{ Water supply and miscellaneous services }} & 6.00 & 6.30 & 7.50 & 6.40 & 6.10 & 9.50 & 8.60 \\
\hline & & & & & & & & & & \\
\hline & & relatir & $g$ to the dwelling & 6.20 & 7.10 & 6.80 & 6.20 & 6.50 & 7.60 & 9.20 \\
\hline & \multirow[t]{4}{*}{4.4} & \multicolumn{2}{|c|}{ Electricity, gas and other fuels } & 15.90 & 16.70 & 16.80 & 16.30 & 17.70 & 17.60 & 15.70 \\
\hline & & 4.4.1 & Electricity & 7.80 & 7.80 & 8.00 & 7.70 & 8.40 & 8.60 & 7.60 \\
\hline & & 4.4 .2 & Gas & 7.80 & 8.30 & 8.20 & 7.60 & 8.50 & 7.20 & 8.10 \\
\hline & & 4.4 .3 & Other fuels & {$[0.30]$} & 0.60 & 0.60 & 0.90 & 0.80 & 1.80 & 0.00 \\
\hline
\end{tabular}

Note: The commodity and service categories are not comparable to those in publications before 2001-02.

The numbering system is sequential, it does not use actual COICOP codes.

Please see page xiii for symbols and conventions used in this report.

This table is based on a three year average.

1 Excluding mortgage interest payments, council tax and Northern Ireland rates.

2 The figure included in total expenditure is net rent as opposed to gross rent. 


\section{Table A37}

\section{Detailed household expenditure by UK countries and Government Office Regions, 2006-2008 (cont.)}

based on weighted data and including children's expenditure

\begin{tabular}{|c|c|c|c|c|c|c|c|c|c|c|}
\hline & & & & $\begin{array}{r}\text { South } \\
\text { East }\end{array}$ & $\begin{array}{r}\text { South } \\
\text { West }\end{array}$ & England & Wales & Scotland & $\begin{array}{l}\text { Northern } \\
\text { Ireland }\end{array}$ & $\begin{array}{r}\text { United } \\
\text { Kingdom }\end{array}$ \\
\hline \multicolumn{5}{|c|}{ Commodity or service } & \multicolumn{6}{|c|}{ Average weekly household expenditure (f) } \\
\hline \multirow[t]{10}{*}{2} & \multicolumn{3}{|c|}{ Alcoholic drink, tobacco \& narcotics } & 10.70 & 10.30 & 10.80 & 11.60 & 12.30 & 13.80 & 11.00 \\
\hline & \multirow[t]{6}{*}{2.1} & \multicolumn{2}{|c|}{ Alcoholic drinks } & 6.90 & 6.50 & 6.40 & 6.30 & 6.50 & 6.00 & 6.40 \\
\hline & & 2.1 .1 & Spirits and liqueurs (brought home) & 1.20 & 1.10 & 1.20 & 1.20 & 1.80 & 1.40 & 1.20 \\
\hline & & 2.1 .2 & Wines, fortified wines (brought home) & 3.90 & 3.60 & 3.40 & 3.20 & 2.90 & 2.80 & 3.30 \\
\hline & & 2.1 .3 & Beer, lager, ciders and perry & & & & & & & \\
\hline & & & (brought home) & 1.70 & 1.60 & 1.80 & 1.90 & 1.70 & 1.70 & 1.80 \\
\hline & & 2.1 .4 & Alcopops (brought home) & 0.00 & 0.10 & 0.10 & [0.10] & 0.10 & 0.10 & 0.10 \\
\hline & \multirow[t]{3}{*}{2.2} & Tobac & co and narcotics & 3.80 & 3.80 & 4.30 & 5.30 & 5.80 & 7.80 & 4.60 \\
\hline & & 2.2 .1 & Cigarettes & 3.20 & 3.00 & 3.80 & 4.50 & 5.10 & 7.50 & 4.00 \\
\hline & & 2.2 .2 & $\begin{array}{l}\text { Cigars, other tobacco } \\
\text { products and narcotics }\end{array}$ & 0.50 & 0.80 & 0.60 & 0.80 & 0.70 & 0.40 & 0.60 \\
\hline \multirow[t]{14}{*}{3} & \multicolumn{3}{|c|}{ Clothing \& footwear } & 21.10 & 19.90 & 21.90 & 19.40 & 23.30 & 34.50 & 22.20 \\
\hline & \multirow[t]{12}{*}{3.1} & \multicolumn{2}{|c|}{ Clothing } & 17.20 & 16.20 & 17.60 & 15.80 & 19.30 & 27.70 & 17.90 \\
\hline & & 3.1 .1 & Men's outer garments & 4.20 & 3.70 & 4.40 & 3.80 & 4.70 & 7.10 & 4.50 \\
\hline & & 3.1 .2 & Men's under garments & 0.40 & 0.40 & 0.40 & 0.30 & 0.30 & 0.50 & 0.40 \\
\hline & & 3.1 .3 & Women's outer garments & 7.70 & 7.50 & 7.80 & 7.50 & 8.70 & 12.80 & 8.00 \\
\hline & & 3.1.4 & Women's under garments & 1.30 & 1.30 & 1.20 & 0.90 & 1.20 & 1.40 & 1.20 \\
\hline & & 3.1 .5 & Boys' outer garments (5-15) & 0.60 & 0.50 & 0.70 & 0.60 & 0.80 & 1.40 & 0.70 \\
\hline & & 3.1 .6 & Girls' outer garments (5-15) & 0.90 & 0.90 & 1.00 & 0.80 & 1.00 & 1.80 & 1.00 \\
\hline & & 3.1.7 & Infants' outer garments (under 5) & 0.60 & 0.60 & 0.60 & 0.50 & 0.70 & 0.90 & 0.70 \\
\hline & & 3.1 .8 & Children's under garments (under 16) & 0.30 & 0.20 & 0.30 & 0.30 & 0.40 & 0.50 & 0.30 \\
\hline & & 3.1 .9 & Accessories & 0.60 & 0.60 & 0.70 & 0.70 & 0.90 & 0.80 & 0.70 \\
\hline & & 3.1 .10 & $\begin{array}{l}\text { Haberdashery, clothing materials } \\
\text { and clothing hire }\end{array}$ & 0.40 & 0.20 & 0.20 & 0.20 & 0.30 & 0.40 & 0.20 \\
\hline & & 3.1.11 & Dry cleaners, laundry and dyeing & 0.30 & 0.30 & 0.30 & 0.20 & 0.20 & 0.30 & 0.30 \\
\hline & 3.2 & Footw & ear & 3.80 & 3.70 & 4.20 & 3.60 & 4.00 & 6.80 & 4.30 \\
\hline \multirow[t]{14}{*}{4} & \multicolumn{3}{|c|}{ Housing (net)' , fuel \& power } & 55.80 & 53.30 & 52.10 & 47.80 & 42.30 & 45.00 & 50.80 \\
\hline & \multirow[t]{6}{*}{4.1} & \multicolumn{2}{|c|}{ Actual rentals for housing } & 33.00 & 26.10 & 32.10 & 24.20 & 21.70 & 20.80 & 30.40 \\
\hline & & 4.1.1 & Gross rent & 32.90 & 26.10 & 32.00 & 24.20 & 21.70 & 20.80 & 30.40 \\
\hline & & 4.1 .2 & less housing benefit, & & & & & & & \\
\hline & & & rebates \& allowances rec'd & 10.60 & 8.80 & 12.20 & 9.20 & 10.30 & 9.00 & 11.80 \\
\hline & & 4.1 .3 & Net rent ${ }^{2}$ & 22.30 & 17.30 & 19.80 & 15.00 & 11.40 & 11.80 & 18.60 \\
\hline & & 4.1.4 & Second dwelling rent & [0.10] & {$[0.00]$} & [0.10] & - & - & {$[0.00]$} & [0.10] \\
\hline & 4.2 & Maint & enance and repair of dwelling & 8.80 & 10.30 & 7.90 & 6.40 & 6.00 & 8.10 & 7.70 \\
\hline & \multirow[t]{2}{*}{4.3} & \multicolumn{2}{|c|}{ Water supply and miscellaneous services } & & & & & & & \\
\hline & & relatir & g to the dwelling & 7.50 & 8.30 & 7.40 & 7.40 & 6.60 & 0.40 & 7.20 \\
\hline & \multirow[t]{4}{*}{4.4} & \multicolumn{2}{|c|}{ Electricity, gas and other fuels } & 17.10 & 17.40 & 16.80 & 18.90 & 18.40 & 24.70 & 17.30 \\
\hline & & 4.4 .1 & Electricity & 8.60 & 8.70 & 8.20 & 8.90 & 9.10 & 9.30 & 8.30 \\
\hline & & 4.4 .2 & Gas & 8.10 & 6.40 & 7.80 & 7.50 & 7.80 & 1.70 & 7.70 \\
\hline & & 4.4.3 & Other fuels & 0.50 & 2.30 & 0.80 & 2.60 & 1.50 & 13.70 & 1.30 \\
\hline
\end{tabular}

Note: The commodity and service categories are not comparable to those in publications before 2001-02.

The numbering system is sequential, it does not use actual COICOP codes.

Please see page xiii for symbols and conventions used in this report.

This table is based on a three year average.

1 Excluding mortgage interest payments, council tax and Northern Ireland rates.

2 The figure included in total expenditure is net rent as opposed to gross rent. 


\section{Table A37}

Detailed household expenditure by UK countries and Government Office Regions, 2006-2008 (cont.)

based on weighted data and including children's expenditure



Note: The commodity and service categories are not comparable to those in publications before 2001-02. The numbering system is sequential, it does not use actual COICOP codes.

Please see page xiii for symbols and conventions used in this report.

This table is based on a three year average. 


\section{Table A37}

\section{Detailed household expenditure by UK countries and Government Office Regions, 2006-2008 (cont.)}

based on weighted data and including children's expenditure

\begin{tabular}{|c|c|c|c|c|c|c|c|c|c|c|}
\hline & & & & $\begin{array}{r}\text { South } \\
\text { East }\end{array}$ & $\begin{array}{r}\text { South } \\
\text { West }\end{array}$ & England & Wales & Scotland & $\begin{array}{l}\text { Northern } \\
\text { Ireland }\end{array}$ & $\begin{array}{r}\text { United } \\
\text { Kingdom }\end{array}$ \\
\hline \multicolumn{5}{|c|}{ Commodity or service } & \multicolumn{6}{|c|}{ Average weekly household expenditure (f) } \\
\hline 5 & \multicolumn{3}{|c|}{ Household goods \& services } & 32.90 & 31.50 & 30.50 & 25.70 & 30.00 & 32.80 & 30.30 \\
\hline & \multirow[t]{4}{*}{5.1} & \multirow{2}{*}{\multicolumn{2}{|c|}{$\begin{array}{l}\text { Furniture and furnishings, carpets and } \\
\text { other floor coverings }\end{array}$}} & & & & & & & \\
\hline & & & & 17.40 & 16.20 & 16.90 & 10.90 & 17.50 & 18.50 & 16.70 \\
\hline & & 5.1 .1 & Furniture and furnishings & 13.10 & 12.70 & 13.00 & 7.80 & 13.00 & 14.10 & 12.80 \\
\hline & & 5.1 .2 & Floor coverings & 4.20 & 3.40 & 3.90 & 3.00 & 4.50 & 4.40 & 3.90 \\
\hline & 5.2 & \multicolumn{2}{|c|}{ Household textiles } & 2.00 & 1.40 & 1.70 & 1.80 & 1.70 & 1.90 & 1.70 \\
\hline & 5.3 & \multicolumn{2}{|c|}{ Household appliances } & 3.80 & 3.90 & 3.30 & 4.40 & 2.40 & 3.30 & 3.30 \\
\hline & 5.4 & \multicolumn{2}{|c|}{ Glassware, tableware and household utensils } & 1.70 & 1.80 & 1.50 & 1.50 & 1.50 & 1.30 & 1.50 \\
\hline & 5.5 & \multicolumn{2}{|c|}{ Tools and equipment for house and garden } & 2.30 & 2.50 & 2.10 & 2.60 & 2.10 & 2.40 & 2.10 \\
\hline & 5.6 & \multicolumn{2}{|c|}{ Goods and services for routine household } & & & & & & & \\
\hline & & \multicolumn{2}{|c|}{ maintenance } & 5.80 & 5.70 & 5.10 & 4.50 & 4.90 & 5.30 & 5.00 \\
\hline & & 5.6 .1 & Cleaning materials & 2.40 & 2.30 & 2.20 & 2.10 & 2.00 & 2.50 & 2.20 \\
\hline & & 5.6 .2 & Household goods and hardware & 1.40 & 1.10 & 1.10 & 1.10 & 1.00 & 1.20 & 1.10 \\
\hline & & 5.6 .3 & Domestic services, carpet cleaning and & & & & & & & \\
\hline & & & hire/repair of furniture/furnishings & 1.90 & 2.30 & 1.70 & 1.30 & 1.90 & 1.60 & 1.70 \\
\hline \multirow[t]{7}{*}{6} & \multicolumn{3}{|c|}{ Health } & 6.40 & 6.00 & 5.80 & 3.50 & 4.50 & 4.40 & 5.60 \\
\hline & 6.1 & \multirow{2}{*}{\multicolumn{2}{|c|}{$\begin{array}{l}\text { Medical products, appliances and equipment } \\
\text { 6.1.1 Medicines, prescriptions, healthcare }\end{array}$}} & 3.90 & 3.30 & 3.20 & 2.50 & 2.30 & 3.20 & 3.10 \\
\hline & & & & & & & & & & \\
\hline & & & products and equipment & 2.00 & 1.70 & 1.70 & 1.40 & 1.40 & 2.10 & 1.70 \\
\hline & & \multirow[t]{2}{*}{6.1 .2} & Spectacles, lenses, accessories & & & & & & & \\
\hline & & & and repairs & 1.80 & 1.60 & 1.50 & 1.10 & 0.80 & 1.20 & 1.40 \\
\hline & 6.2 & \multicolumn{2}{|c|}{ Hospital services } & 2.50 & 2.60 & 2.60 & 1.00 & 2.30 & 1.20 & 2.40 \\
\hline \multirow[t]{16}{*}{7} & \multicolumn{3}{|c|}{ Transport } & 76.40 & 65.60 & 62.50 & 56.20 & 59.90 & 62.80 & 62.00 \\
\hline & \multirow[t]{5}{*}{7.1} & \multicolumn{2}{|c|}{ Purchase of vehicles } & 30.70 & 23.60 & 22.40 & 18.70 & 24.30 & 19.30 & 22.30 \\
\hline & & 7.1.1 & Purchase of new cars and vans & 9.90 & 7.30 & 7.30 & {$[8.20]$} & 8.80 & 8.20 & 7.50 \\
\hline & & 7.1.2 & Purchase of second hand cars or vans & 20.20 & 15.70 & 14.30 & 10.00 & 14.90 & 10.90 & 14.10 \\
\hline & & \multirow[t]{2}{*}{ 7.1.3 } & Purchase of motorcycles & & & & & & & \\
\hline & & & and other vehicles & 0.60 & 0.60 & 0.70 & {$[0.50]$} & 0.60 & {$[0.20]$} & 0.70 \\
\hline & \multirow[t]{5}{*}{7.2} & Opera & tion of personal transport & 34.70 & 33.90 & 29.60 & 30.70 & 27.20 & 34.60 & 29.50 \\
\hline & & 7.2 .1 & Spares and accessories & 2.80 & 2.30 & 2.10 & 2.10 & 1.80 & 2.60 & 2.10 \\
\hline & & 7.2 .2 & Petrol, diesel and other motor oils & 21.00 & 21.10 & 18.80 & 21.70 & 18.40 & 25.10 & 19.10 \\
\hline & & 7.2 .3 & Repairs and servicing & 8.10 & 7.90 & 6.20 & 5.30 & 5.20 & 5.20 & 6.10 \\
\hline & & 7.2.4 & Other motoring costs & 2.80 & 2.60 & 2.40 & 1.60 & 1.90 & 1.70 & 2.30 \\
\hline & 7.3 & Transp & ort services & 10.90 & 8.10 & 10.60 & 6.80 & 8.40 & 8.90 & 10.10 \\
\hline & & 7.3.1 & Rail and tube fares & 4.30 & 1.80 & 2.60 & 0.80 & 1.80 & 0.70 & 2.30 \\
\hline & & 7.3 .2 & Bus and coach fares & 0.80 & 1.10 & 1.30 & 1.00 & 1.70 & 1.00 & 1.30 \\
\hline & & 7.3 .3 & Combined fares & 0.90 & {$[0.10]$} & 1.40 & {$[0.00]$} & {$[0.20]$} & {$[0.10]$} & 1.20 \\
\hline & & 7.3 .4 & Other travel and transport & 4.90 & 5.20 & 5.40 & 4.90 & 4.60 & 7.20 & 5.30 \\
\hline 8 & Com & imunic & ation & 11.90 & 11.70 & 11.90 & 10.60 & 11.00 & 14.60 & 11.80 \\
\hline & & Postal & services & 0.60 & 0.60 & 0.50 & 0.50 & 0.50 & 0.50 & 0.50 \\
\hline & 8.2 & Teleph & ione and telefax equipment & 0.40 & 0.50 & 0.60 & 0.70 & 0.60 & 0.80 & 0.60 \\
\hline & 8.3 & Teleph & one and telefax services & 10.80 & 10.50 & 10.80 & 9.50 & 9.80 & 13.30 & 10.70 \\
\hline
\end{tabular}

Note: The commodity and service categories are not comparable to those in publications before 2001-02.

The numbering system is sequential, it does not use actual COICOP codes.

Please see page xiii for symbols and conventions used in this report.

This table is based on a three year average. 


\section{Table A37}

\section{Detailed household expenditure by UK countries and Government Office Regions, 2006-2008 (cont.)}

based on weighted data and including children's expenditure

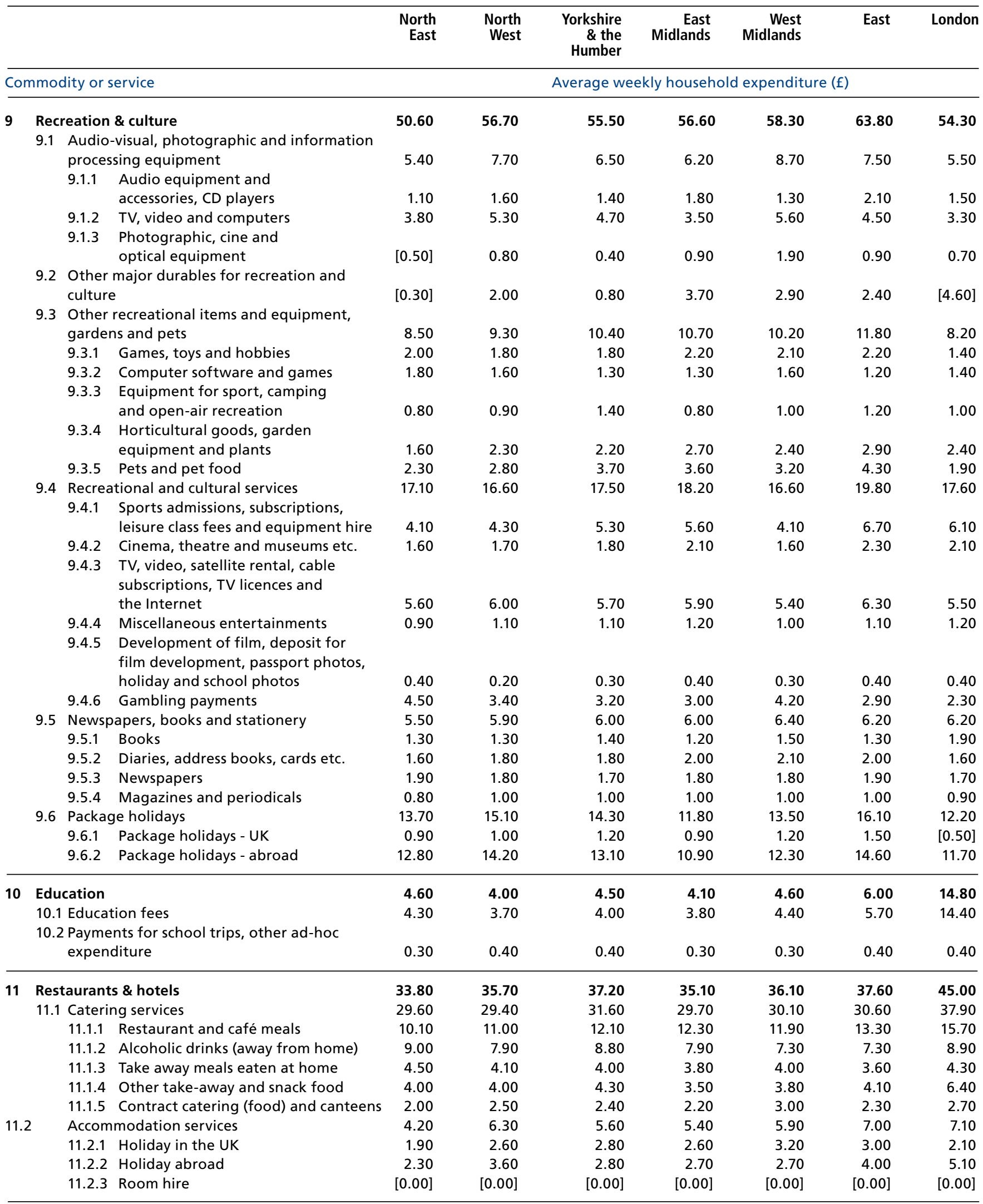

Note: The commodity and service categories are not comparable to those in publications before 2001-02.

The numbering system is sequential, it does not use actual COICOP codes.

Please see page xiii for symbols and conventions used in this report.

This table is based on a three year average. 
Table A37

\section{Detailed household expenditure by UK countries and Government Office Regions, 2006-2008 (cont.)}

based on weighted data and including children's expenditure

\begin{tabular}{|c|c|c|c|c|c|c|c|c|c|c|}
\hline \multirow{2}{*}{\multicolumn{4}{|c|}{ Commodity or service }} & \multirow[t]{2}{*}{$\begin{array}{r}\text { South } \\
\text { East }\end{array}$} & \multirow[t]{2}{*}{$\begin{array}{r}\text { South } \\
\text { West }\end{array}$} & \multirow[t]{2}{*}{ England } & \multirow[t]{2}{*}{ Wales } & \multirow[t]{2}{*}{ Scotland } & \multirow[t]{2}{*}{$\begin{array}{l}\text { Northern } \\
\text { Ireland }\end{array}$} & \multirow[t]{2}{*}{$\begin{array}{r}\text { United } \\
\text { Kingdom }\end{array}$} \\
\hline & & & & & & & & & & \\
\hline \multirow[t]{34}{*}{9} & \multirow{6}{*}{$\begin{array}{l}\text { Recr } \\
9.1\end{array}$} & reation & \& culture & 61.70 & 63.60 & 58.30 & 58.20 & 59.20 & 55.90 & 58.30 \\
\hline & & $\begin{array}{l}\text { Audio- } \\
\text { process }\end{array}$ & $\begin{array}{l}\text {-visual, photographic and information } \\
\text { sing equipment }\end{array}$ & 8.00 & 9.10 & 7.30 & 6.20 & 8.10 & 7.20 & 7.30 \\
\hline & & 9.1 .1 & Audio equipment and accessories, & & & & & & & \\
\hline & & & CD players & 1.70 & 1.60 & 1.60 & 1.40 & 1.40 & 1.40 & 1.60 \\
\hline & & 9.1 .2 & TV, video and computers & 5.40 & 6.80 & 4.80 & 4.40 & 6.30 & 5.30 & 4.90 \\
\hline & & 9.1 .3 & $\begin{array}{l}\text { Photographic, cine and optical } \\
\text { equipment }\end{array}$ & 0.80 & 0.70 & 0.80 & 0.50 & 0.40 & 0.50 & 0.80 \\
\hline & 9.2 & $\begin{array}{l}\text { Other } r \\
\text { culture }\end{array}$ & major durables for recreation and & 2.00 & 2.00 & 2.50 & 7.30 & 2.30 & 1.50 & 2.70 \\
\hline & \multirow[t]{9}{*}{9.3} & Other $r$ & recreational items and equipment, & & & & & & & \\
\hline & & garden & ns and pets & 12.00 & 11.50 & 10.40 & 10.40 & 10.70 & 11.30 & 10.40 \\
\hline & & 9.3.1 & Games, toys and hobbies & 2.00 & 1.90 & 1.90 & 1.70 & 1.90 & 2.80 & 1.90 \\
\hline & & 9.3 .2 & Computer software and games & 1.60 & 1.10 & 1.40 & 1.10 & 2.10 & 1.50 & 1.50 \\
\hline & & 9.3 .3 & Equipment for sport, camping and & & & & & & & \\
\hline & & & open-air recreation & 1.10 & 1.30 & 1.10 & 1.10 & 0.70 & 1.80 & 1.10 \\
\hline & & 9.3 .4 & Horticultural goods, garden equipment & & & & & & & \\
\hline & & & and plants & 3.00 & 3.20 & 2.60 & 2.50 & 3.20 & 2.50 & 2.60 \\
\hline & & 9.3.5 & Pets and pet food & 4.40 & 4.00 & 3.40 & 4.10 & 2.90 & 2.70 & 3.40 \\
\hline & \multirow[t]{10}{*}{9.4} & Recreat & tional and cultural services & 18.40 & 17.20 & 17.70 & 15.60 & 18.30 & 17.60 & 17.60 \\
\hline & & 9.4 .1 & Sports admissions, subscriptions, & & & & & & & \\
\hline & & & leisure class fees and equipment hire & 5.80 & 5.30 & 5.30 & 4.30 & 4.90 & 4.80 & 5.20 \\
\hline & & 9.4 .2 & Cinema, theatre and museums etc. & 2.10 & 2.10 & 2.00 & 1.90 & 2.00 & 2.00 & 2.00 \\
\hline & & 9.4 .3 & $\begin{array}{l}\text { TV, video, satellite rental, cable } \\
\text { subscriptions, TV licences and the }\end{array}$ & & & & & & & \\
\hline & & & Internet & 5.90 & 5.30 & 5.70 & 5.50 & 6.10 & 5.70 & 5.80 \\
\hline & & 9.4 .4 & Miscellaneous entertainments & 1.00 & 1.50 & 1.10 & 0.80 & 0.80 & 1.30 & 1.10 \\
\hline & & 9.4 .5 & $\begin{array}{l}\text { Development of film, deposit for film } \\
\text { development, passport photos, holiday }\end{array}$ & & & & & & & \\
\hline & & & and school photos & 0.50 & 0.30 & 0.40 & 0.20 & 0.30 & 0.40 & 0.40 \\
\hline & & 9.4 .6 & Gambling payments & 3.00 & 2.60 & 3.10 & 3.00 & 4.10 & 3.50 & 3.20 \\
\hline & \multirow[t]{5}{*}{9.5} & Newsp & apers, books and stationery & 7.20 & 6.80 & 6.30 & 6.00 & 6.70 & 6.80 & 6.30 \\
\hline & & 9.5 .1 & Books & 1.90 & 1.50 & 1.50 & 1.30 & 1.50 & 1.20 & 1.50 \\
\hline & & 9.5 .2 & Diaries, address books, cards etc. & 2.30 & 2.20 & 1.90 & 1.90 & 1.90 & 1.80 & 1.90 \\
\hline & & 9.5 .3 & Newspapers & 1.90 & 2.00 & 1.80 & 1.90 & 2.50 & 2.60 & 1.90 \\
\hline & & 9.5 .4 & Magazines and periodicals & 1.10 & 1.10 & 1.00 & 0.90 & 1.00 & 1.10 & 1.00 \\
\hline & \multirow[t]{3}{*}{9.6} & Packag & ge holidays & 14.10 & 17.00 & 14.20 & 12.60 & 13.20 & 11.40 & 14.00 \\
\hline & & 9.6 .1 & Package holidays - UK & 1.10 & 1.00 & 1.00 & 1.00 & 0.60 & 0.60 & 1.00 \\
\hline & & 9.6 .2 & Package holidays - abroad & 12.90 & 16.00 & 13.20 & 11.70 & 12.60 & 10.80 & 13.00 \\
\hline \multirow[t]{4}{*}{10} & \multicolumn{3}{|c|}{ Education } & 7.80 & 8.90 & 7.10 & 5.00 & 4.30 & 4.60 & 6.70 \\
\hline & \multirow{2}{*}{\multicolumn{3}{|c|}{$\begin{array}{l}\text { 10.1 Education fees } \\
\text { 10.2 Payments for school trips, other ad-hoc }\end{array}$}} & 7.50 & 8.60 & 6.70 & 4.70 & 4.10 & 4.00 & 6.30 \\
\hline & & & & & $0 \wedge 0$ & 030 & & 020 & 060 & 030 \\
\hline & & & & & & & & & & \\
\hline 11 & Resta & taurants & s \& hotels & 39.30 & 36.70 & 37.90 & 30.90 & 35.40 & 44.30 & 37.50 \\
\hline & 11.1 & Caterin & ng services & 31.10 & 28.80 & 31.30 & 28.30 & 30.70 & 39.80 & 31.30 \\
\hline & & 11.1.1 & Restaurant and café meals & 14.30 & 13.10 & 12.90 & 11.20 & 12.10 & 15.50 & 12.80 \\
\hline & & 11.1.2 & Alcoholic drinks (away from home) & 7.10 & 7.40 & 7.90 & 8.00 & 7.20 & 8.60 & 7.80 \\
\hline & & 11.1.3 & Take away meals eaten at home & 3.60 & 3.20 & 3.90 & 3.60 & 4.00 & 6.80 & 3.90 \\
\hline & & 11.1.4 & Other take-away and snack food & 3.90 & 3.10 & 4.20 & 3.80 & 4.20 & 5.90 & 4.20 \\
\hline & & 11.1.5 & Contract catering (food) and canteens & 2.20 & 2.00 & 2.40 & 1.70 & 3.30 & 3.00 & 2.40 \\
\hline & 11.2 & Accomr & imodation services & 8.10 & 7.90 & 6.60 & 2.60 & 4.70 & 4.50 & 6.20 \\
\hline & & 11.2.1 & Holiday in the UK & 3.30 & 3.30 & 2.80 & 1.10 & 2.10 & 0.90 & 2.60 \\
\hline & & 11.2 .2 & Holiday abroad & 4.80 & 4.50 & 3.80 & 1.50 & 2.60 & 3.60 & 3.60 \\
\hline & & 11.2 .3 & Room hire & {$[0.00]$} & {$[0.00]$} & 0.00 & {$[0.00]$} & {$[0.00]$} & {$[0.00]$} & 0.00 \\
\hline
\end{tabular}

Note: The commodity and service categories are not comparable to those in publications before 2001-02.

The numbering system is sequential, it does not use actual COICOP codes.

Please see page xiii for symbols and conventions used in this report.

This table is based on a three year average. 


\section{Table A37}

\section{Detailed household expenditure by UK countries and Government Office Regions, 2006-2008 (cont.)}

based on weighted data and including children's expenditure

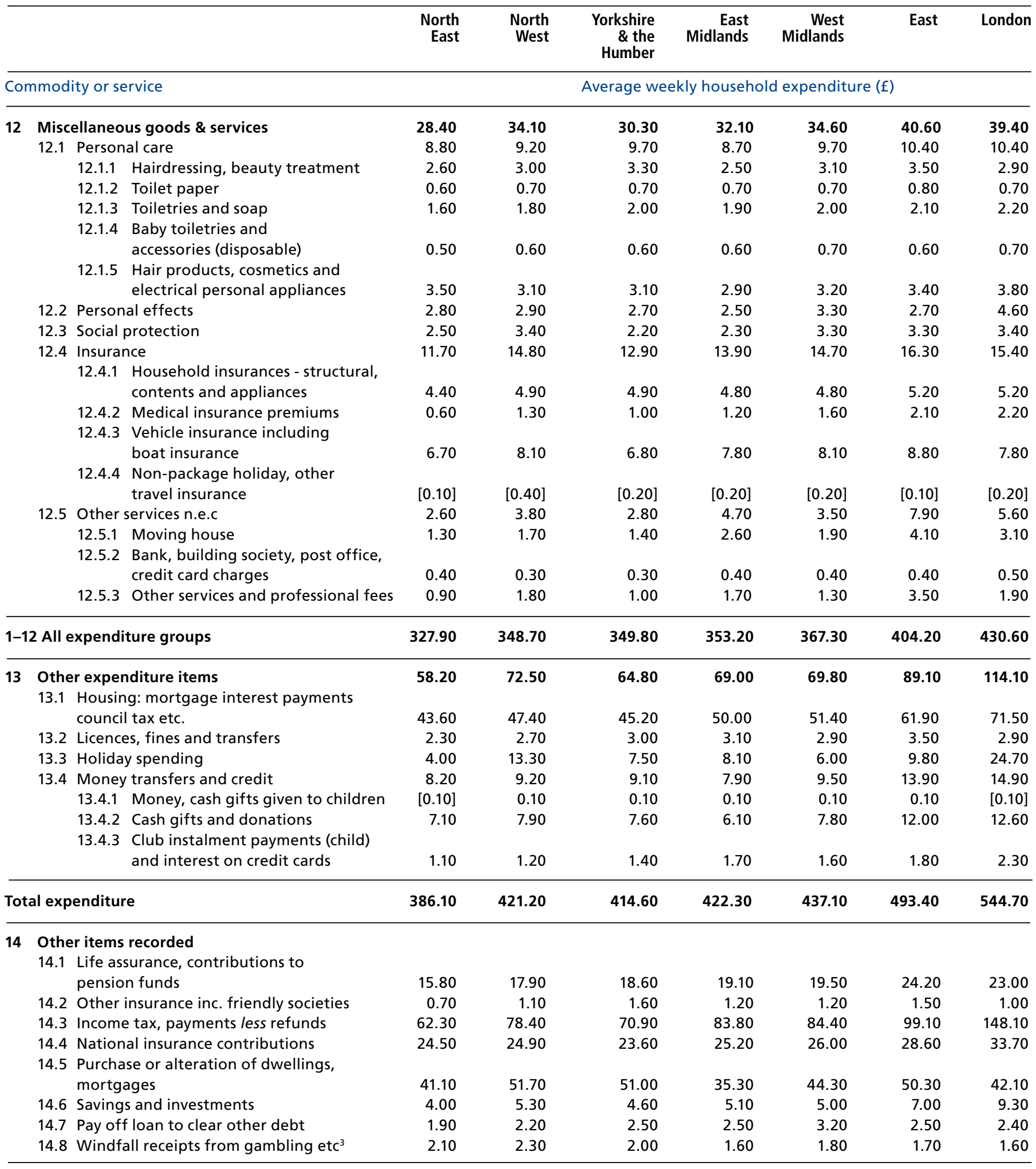

Note: $\quad$ The commodity and service categories are not comparable to those in publications before 2001-02.

The numbering system is sequential, it does not use actual COICOP codes.

Please see page xiii for symbols and conventions used in this report.

This table is based on a three year average.

3 Expressed as an income figure as opposed to an expenditure figure. 
Table A37

\section{Detailed household expenditure by UK countries and Government Office Regions, 2006-2008 (cont.)}

based on weighted data and including children's expenditure

\begin{tabular}{|c|c|c|c|c|c|c|c|c|c|}
\hline & & & $\begin{array}{r}\text { South } \\
\text { East }\end{array}$ & $\begin{array}{l}\text { South } \\
\text { West }\end{array}$ & England & Wales & Scotland & $\begin{array}{l}\text { Northern } \\
\text { Ireland }\end{array}$ & $\begin{array}{r}\text { United } \\
\text { Kingdom }\end{array}$ \\
\hline \multicolumn{5}{|c|}{ Commodity or service } & \multicolumn{5}{|c|}{ Average weekly household expenditure (f) } \\
\hline \multirow[t]{20}{*}{12} & Misc & ellaneous goods \& services & 42.00 & 35.20 & 36.10 & 29.70 & 32.50 & 39.20 & 35.50 \\
\hline & 12.1 & Personal care & 10.80 & 9.40 & 9.80 & 8.70 & 9.80 & 12.30 & 9.80 \\
\hline & & 12.1.1 Hairdressing, beauty treatment & 3.80 & 2.80 & 3.10 & 2.60 & 3.40 & 4.00 & 3.10 \\
\hline & & 12.1.2 Toilet paper & 0.80 & 0.80 & 0.70 & 0.70 & 0.70 & 0.90 & 0.70 \\
\hline & & 12.1.3 Toiletries and soap & 2.30 & 2.10 & 2.00 & 2.00 & 1.90 & 2.50 & 2.00 \\
\hline & & 12.1.4 Baby toiletries and & & & & & & & \\
\hline & & accessories (disposable) & 0.70 & 0.50 & 0.60 & 0.50 & 0.50 & 0.70 & 0.60 \\
\hline & & $\begin{array}{l}\text { 12.1.5 Hair products, cosmetics and } \\
\text { electrical personal appliances }\end{array}$ & 3.40 & 3.20 & 3.30 & 2.90 & 3.40 & 4.20 & 3.30 \\
\hline & 12.2 & Personal effects & 4.30 & 2.90 & 3.30 & 2.30 & 3.70 & 3.00 & 3.30 \\
\hline & 12.3 & Social protection & 3.40 & 2.50 & 3.00 & 2.50 & 2.50 & 3.40 & 3.00 \\
\hline & 12.4 & Insurance & 17.00 & 15.00 & 15.00 & 13.10 & 12.20 & 15.70 & 14.60 \\
\hline & & $\begin{array}{l}\text { 12.4.1 Household insurances - structural, } \\
\text { contents and appliances }\end{array}$ & 5.50 & 5.10 & 5.00 & 4.70 & 4.90 & 4.40 & 5.00 \\
\hline & & 12.4.2 Medical insurance premiums & 2.60 & 2.00 & 1.70 & 0.80 & 1.10 & 1.00 & 1.60 \\
\hline & & 12.4.3 Vehicle insurance including & 870 & 770 & 790 & 750 & 600 & 1020 & 780 \\
\hline & & 12.4.4 Non-package holiday, other & 0.10 & 1.10 & & & 0.00 & 10.20 & \\
\hline & & travel insurance & 0.20 & {$[0.30]$} & 0.20 & {$[0.10]$} & {$[0.20]$} & {$[0.10]$} & 0.20 \\
\hline & 12.5 & Other services n.e.c & 6.40 & 5.30 & 5.00 & 3.10 & 4.20 & 4.90 & 4.80 \\
\hline & & 12.5.1 Moving house & 4.90 & 3.30 & 2.90 & 1.60 & 2.40 & 1.50 & 2.70 \\
\hline & & $\begin{array}{l}\text { 12.5.2 Bank, building society, post office, } \\
\text { credit card charges }\end{array}$ & 0.50 & 0.50 & 0.40 & 0.30 & 0.40 & 0.50 & 0.40 \\
\hline & & 12.5.3 Other services and professional fees & 1.00 & 1.50 & 1.70 & 1.10 & 1.40 & 2.90 & 1.70 \\
\hline \multicolumn{3}{|c|}{ 1-12 All expenditure groups } & 417.50 & 392.50 & 383.20 & 346.30 & 361.70 & 407.30 & 380.00 \\
\hline \multirow[t]{8}{*}{13} & Othe & er expenditure items & 94.80 & 76.70 & 82.00 & 60.40 & 71.10 & 72.40 & 79.70 \\
\hline & 13.1 & $\begin{array}{l}\text { Housing: mortgage interest payments, } \\
\text { council tax etc. }\end{array}$ & 67.70 & 54.80 & 56.60 & 41.00 & 49.10 & 39.80 & 54.70 \\
\hline & 13.2 & Licences, fines and transfers & 3.50 & 3.60 & 3.10 & 3.00 & 2.60 & 3.30 & 3.10 \\
\hline & 13.3 & Holiday spending & 12.30 & 8.60 & 11.60 & 8.70 & 8.50 & 13.50 & 11.20 \\
\hline & 13.4 & Money transfers and credit & 11.30 & 9.80 & 10.80 & 7.80 & 10.80 & 15.80 & 10.70 \\
\hline & & 13.4.1 Money, cash gifts given to children & 0.10 & 0.10 & 0.10 & {$[0.10]$} & 0.10 & 0.20 & 0.10 \\
\hline & & 13.4.2 Cash gifts and donations & 9.20 & 7.70 & 8.90 & 6.30 & 9.10 & 14.70 & 9.00 \\
\hline & & $\begin{array}{l}\text { 13.4.3 Club instalment payments (child) } \\
\text { and interest on credit cards }\end{array}$ & 2.00 & 2.00 & 1.70 & 1.40 & 1.70 & 0.90 & 1.70 \\
\hline \multicolumn{3}{|c|}{ Total expenditure } & 512.30 & 469.20 & 465.20 & 406.70 & 432.80 & 479.70 & 459.70 \\
\hline \multirow[t]{11}{*}{14} & Othe & er items recorded & & & & & & & \\
\hline & 14.1 & Life assurance, contributions to & & & & & & & \\
\hline & & pension funds & 23.20 & 23.00 & 20.90 & 17.90 & 20.60 & 17.30 & 20.60 \\
\hline & 14.2 & Other insurance inc. friendly societies & 1.60 & 1.50 & 1.30 & 0.70 & 1.20 & 0.90 & 1.20 \\
\hline & 14.3 & Income tax, payments less refunds & 120.20 & 85.70 & 97.40 & 68.60 & 82.60 & 72.50 & 94.00 \\
\hline & 14.4 & National insurance contributions & 29.20 & 25.00 & 27.20 & 22.80 & 25.80 & 23.50 & 26.80 \\
\hline & 14.5 & Purchase or alteration of dwellings, & & & & & & & \\
\hline & & mortgages & 53.70 & 41.30 & 46.50 & 34.10 & 49.40 & 33.60 & 45.70 \\
\hline & 14.6 & Savings and investments & 8.20 & 10.20 & 6.90 & 3.40 & 6.40 & 5.90 & 6.60 \\
\hline & 14.7 & Pay off loan to clear other debt & 3.30 & 2.60 & 2.60 & 1.80 & 2.10 & 0.50 & 2.50 \\
\hline & 14.8 & Windfall receipts from gambling etc ${ }^{3}$ & 2.10 & 1.10 & 1.80 & 1.50 & 2.10 & 2.20 & 1.90 \\
\hline
\end{tabular}

Note: The commodity and service categories are not comparable to those in publications before 2001-02.

The numbering system is sequential, it does not use actual COICOP codes.

Please see page xiii for symbols and conventions used in this report.

This table is based on a three year average.

3 Expressed as an income figure as opposed to an expenditure figure. 


\section{Table A38}

Household expenditure by urban/rural areas (GB)'1, 2006-2008

based on weighted data and including children's expenditure

\begin{tabular}{lrr} 
& Urban & Rural \\
\hline Average number of weighted households (thousands) & 19,540 & 5,300 \\
Total number of households in sample (over 3 years) & 13,070 & 3,810 \\
Total number of persons in sample (over 3 years) & 30,900 & 8,860 \\
Total number of adults in sample (over 3 years) & 23,590 & 6,940 \\
Weighted average number of persons per household & 2.4 & 2.3
\end{tabular}

Commodity or service

Average weekly household expenditure (f)

\begin{tabular}{llrr}
\hline 1 & Food \& non-alcoholic drinks & 47.20 & 51.80 \\
2 & Alcoholic drinks, tobacco \& narcotics & 10.70 & 11.80 \\
3 & Clothing \& footwear & 22.00 & 21.40 \\
4 & Housing (net)', fuel \& power & 51.00 & 50.70 \\
5 & Household goods \& services & 28.70 & 35.90 \\
6 & Health & 5.20 & 6.90 \\
7 & Transport & 58.10 & 76.10 \\
8 & Communication & 11.80 & 11.50 \\
9 & Recreation \& culture & 56.00 & 67.40 \\
10 & Education & 6.20 & 8.70 \\
11 & Restaurants \& hotels & 36.80 & 39.20 \\
12 & Miscellaneous goods \& services & 34.40 & 39.30 \\
& & & 420.60 \\
$1-12$ & All expenditure groups & 368.10 & 84.80 \\
13 & Other expenditure items & 505.40 \\
\hline Total expenditure & 78.60 & 219.40 \\
\hline Average weekly expenditure per person (f) & 446.70 & \\
Total expenditure & & \\
\hline
\end{tabular}

Note: The commodity and service categories are not comparable to those in publications before 2001-02.

Please see page xiii for symbols and conventions used in this report.

This table is based on a three year average.

1 Combined urban/rural classification for England \& Wales and Scotland - see definitions in Appendix B.

2 Excludes mortgage interest payments and council tax. 


\section{Table A39}

\section{Government Office Regions of the United Kingdom}

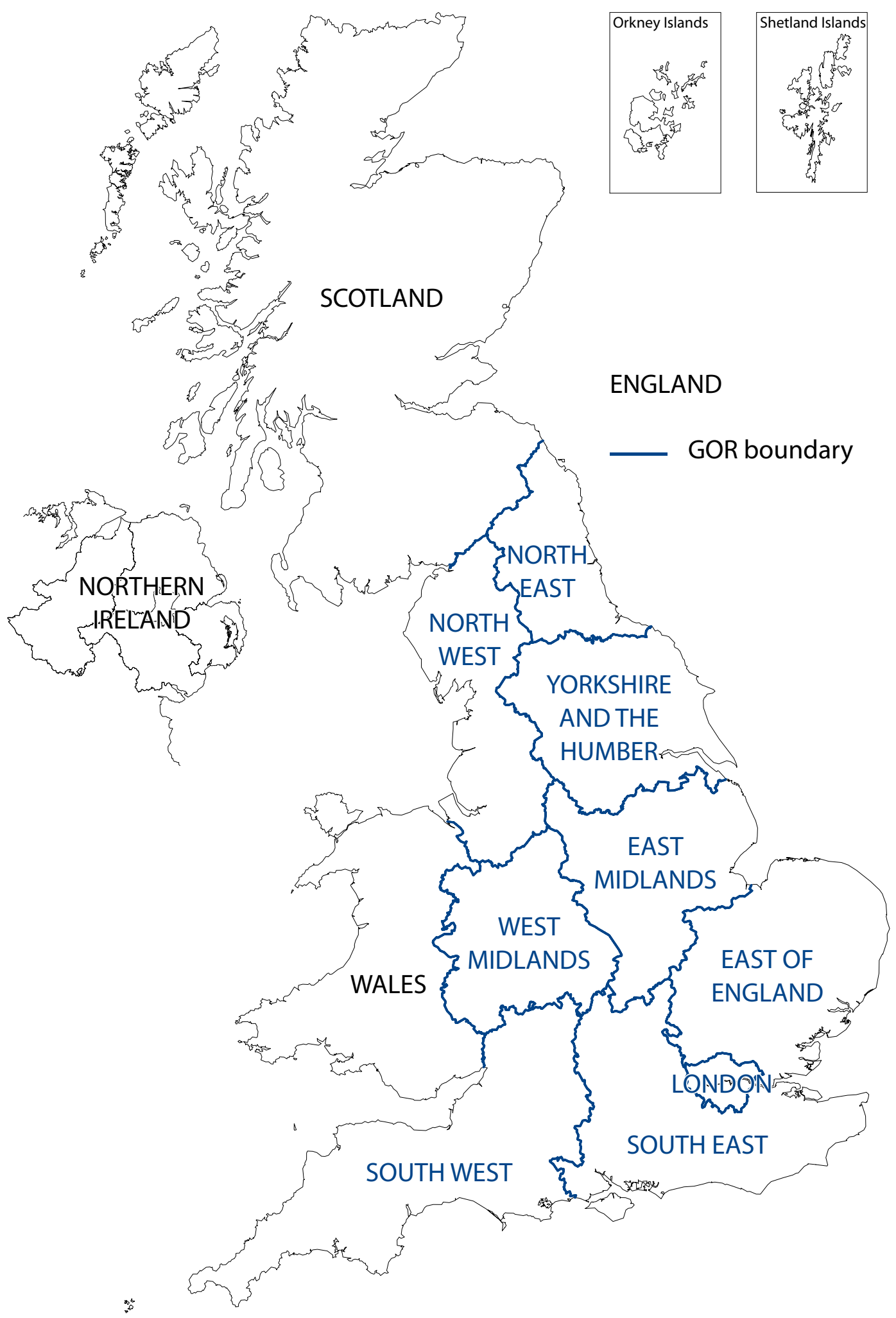




\section{Table A40}

Income and source of income by household composition, 2008

based on weighted data

\begin{tabular}{|c|c|c|c|c|c|c|c|c|c|c|}
\hline \multirow{3}{*}{ Composition of household } & \multirow{3}{*}{$\begin{array}{r}\begin{array}{r}\text { Weighted } \\
\text { number } \\
\text { of house- } \\
\text { holds }\end{array} \\
(000 \mathrm{~s})\end{array}$} & \multirow{3}{*}{$\begin{array}{r}\begin{array}{r}\text { Number } \\
\text { of house- } \\
\text { holds } \\
\text { in the } \\
\text { sample }\end{array} \\
\text { Number }\end{array}$} & \multicolumn{2}{|c|}{$\begin{array}{l}\text { Weekly } \\
\text { household } \\
\text { income }\end{array}$} & \multicolumn{6}{|c|}{ Source of income } \\
\hline & & & $\begin{array}{l}\text { Dispo- } \\
\text { sable }\end{array}$ & Gross & $\begin{array}{r}\text { Wages } \\
\text { and } \\
\text { salaries }\end{array}$ & $\begin{array}{r}\text { Self } \\
\text { employ- } \\
\text { ment }\end{array}$ & $\begin{array}{l}\text { Invest- } \\
\text { ments }\end{array}$ & $\begin{array}{r}\text { Annuities } \\
\text { and } \\
\text { pensions }^{1}\end{array}$ & $\begin{array}{r}\text { Social } \\
\text { security } \\
\text { benefits }^{2}\end{array}$ & $\begin{array}{r}\text { other } \\
\text { sources }\end{array}$ \\
\hline & & & $f$ & $f$ & \multicolumn{6}{|c|}{ Percentage of gross weekly household income } \\
\hline $\begin{array}{l}\text { All households } \\
\text { Composition of household }\end{array}$ & 25,690 & 5,850 & 582 & 713 & 67 & 9 & 4 & 7 & 12 & 1 \\
\hline $\begin{array}{l}\text { One adult } \\
\quad \text { Retired households mainly }\end{array}$ & 7,490 & 1,620 & 280 & 333 & 52 & 6 & 3 & 13 & 25 & 0 \\
\hline dependent on state pensions ${ }^{3}$ & 970 & 200 & 158 & 159 & - & - & 2 & 3 & 95 & [0] \\
\hline Other retired households & 2,660 & 570 & 226 & 244 & - & - & 7 & 39 & 54 & 0 \\
\hline Non-retired households & 3,860 & 850 & 347 & 438 & 77 & 9 & 2 & 4 & 8 & 0 \\
\hline One adult, one child & 830 & 200 & 297 & 337 & 56 & [6] & 1 & [1] & 32 & 3 \\
\hline One adult, two or more children & 650 & 180 & 315 & 346 & 40 & [3] & 0 & [0] & 52 & 4 \\
\hline $\begin{array}{l}\text { One man and one woman } \\
\text { Retired households mainly }\end{array}$ & 7,590 & 1,860 & 607 & 746 & 62 & 8 & 4 & 13 & 13 & 0 \\
\hline dependent on state pensions ${ }^{3}$ & 540 & 150 & 247 & 249 & [0] & {$[0]$} & 2 & 7 & 91 & [0] \\
\hline Other retired households & 2,000 & 530 & 419 & 466 & 5 & [1] & 9 & 42 & 43 & 0 \\
\hline Non-retired households & 5,050 & 1,170 & 720 & 910 & 75 & 10 & 3 & 7 & 4 & 0 \\
\hline Two men or two women & 560 & 110 & 554 & 677 & 79 & [5] & 1 & 4 & 9 & [2] \\
\hline Two men or two women with children & 120 & 30 & 392 & 434 & 56 & [5] & {$[0]$} & [0] & 36 & [2] \\
\hline One man one woman, one child & 1,860 & 420 & 689 & 869 & 79 & 11 & 2 & 1 & 6 & 0 \\
\hline One man one woman, two children & 2,150 & 540 & 884 & 1,098 & 73 & 18 & 2 & [0] & 6 & 1 \\
\hline One man one woman, three children & 640 & 160 & 1,626 & 2,071 & 71 & 5 & 18 & {$[0]$} & 5 & 1 \\
\hline Two adults, four or more children & 180 & 50 & 658 & 785 & 55 & [14] & [2] & [0] & 28 & 1 \\
\hline Three adults & 1,610 & 320 & 819 & 1,005 & 73 & 9 & 3 & 7 & 8 & 1 \\
\hline Three adults, one or more children & 830 & 180 & 802 & 978 & 77 & 10 & 2 & 1 & 10 & 1 \\
\hline Four or more adults & 870 & 150 & 985 & 1,213 & 79 & 9 & 1 & 2 & 6 & 3 \\
\hline $\begin{array}{l}\text { Four or more adults, } \\
\text { One or more children }\end{array}$ & 290 & 60 & 851 & 1,015 & 74 & [6] & 1 & [2] & 16 & [1] \\
\hline
\end{tabular}

Note: Please see page xiii for symbols and conventions used in this report.

1 Other than social security benefits.

2 Excluding housing benefit and council tax benefit (rates rebate in Northern Ireland) - see definitions in Appendix B.

3 Mainly dependent on state pension and not economically active - see defintions in Appendix B.

\section{Table A41}

Income and source of income by age of household reference person, 2008

based on weighted data

\begin{tabular}{|c|c|c|c|c|c|c|c|c|c|c|}
\hline & \multirow[t]{2}{*}{$\begin{array}{r}\text { Weighted } \\
\text { number } \\
\text { of house- } \\
\text { holds }\end{array}$} & \multirow{2}{*}{$\begin{array}{r}\text { Number } \\
\text { of house- } \\
\text { holds } \\
\text { in the } \\
\text { sample }\end{array}$} & \multicolumn{2}{|c|}{$\begin{array}{c}\text { Weekly } \\
\text { household } \\
\text { income }\end{array}$} & \multirow[b]{2}{*}{$\begin{array}{r}\text { Wages } \\
\text { and } \\
\text { salaries }\end{array}$} & \multicolumn{5}{|c|}{ Source of income } \\
\hline & & & $\begin{array}{c}\text { Dispo- } \\
\text { sable }\end{array}$ & Gross & & $\begin{array}{r}\text { Self } \\
\text { employ- } \\
\text { ment }\end{array}$ & $\begin{array}{l}\text { Invest- } \\
\text { ments }\end{array}$ & $\begin{array}{r}\text { Annuities } \\
\text { and } \\
\text { pensions }^{1}\end{array}$ & $\begin{array}{r}\text { Social } \\
\text { security } \\
\text { benefits }^{2}\end{array}$ & $\begin{array}{r}\text { Other } \\
\text { sources }\end{array}$ \\
\hline Age of head of household & (000s) & Number & $f$ & $f$ & & \multicolumn{5}{|c|}{ Percentage of gross weekly household income } \\
\hline 30 to 49 & 9,740 & 2,230 & 751 & 945 & 78 & 11 & 4 & {$[0]$} & 6 & 1 \\
\hline 50 to 64 & 6,750 & 1,590 & 622 & 771 & 67 & 10 & 4 & 9 & 8 & 1 \\
\hline 65 to 74 & 3,140 & 810 & 388 & 434 & 18 & 5 & 6 & 29 & 41 & 1 \\
\hline 75 or over & 3,520 & 760 & 272 & 290 & 3 & [2] & 6 & 32 & 57 & 0 \\
\hline
\end{tabular}

Note: Please see page xiii for symbols and conventions used in this report.

1 Other than social security benefits.

2 Excluding housing benefit and council tax benefit (rates rebate in Northern Ireland) - see definitions in Appendix B. 


\section{Table A42}

Income and source of income by gross income quintile group, 2008

based on weighted data

\begin{tabular}{|c|c|c|c|c|c|c|c|c|c|c|}
\hline & \multirow{2}{*}{$\begin{array}{r}\text { Weighted } \\
\text { number } \\
\text { of house- } \\
\text { holds }\end{array}$} & \multirow{2}{*}{$\begin{array}{r}\text { Number } \\
\text { of house- } \\
\text { holds } \\
\text { in the } \\
\text { sample }\end{array}$} & \multicolumn{2}{|c|}{$\begin{array}{l}\text { Weekly } \\
\text { household } \\
\text { income }\end{array}$} & \multirow[b]{2}{*}{$\begin{array}{l}\text { Wages } \\
\text { and } \\
\text { salaries }\end{array}$} & \multicolumn{5}{|c|}{ Source of income } \\
\hline & & & $\begin{array}{c}\text { Dispo- } \\
\text { sable }\end{array}$ & Gross & & $\begin{array}{r}\text { Self } \\
\text { employ- } \\
\text { ment }\end{array}$ & $\begin{array}{l}\text { Invest- } \\
\text { ments }\end{array}$ & $\begin{array}{r}\text { Annuities } \\
\text { and } \\
\text { pensions }{ }^{1}\end{array}$ & $\begin{array}{r}\text { Social } \\
\text { security } \\
\text { benefits }^{2}\end{array}$ & $\begin{array}{r}\text { Other } \\
\text { sources }\end{array}$ \\
\hline Gross income quintile group & (000s) & Number & $f$ & $f$ & & \multicolumn{5}{|c|}{ Percentage of gross weekly household income } \\
\hline Second quintile group & 5,130 & 1,240 & 285 & 310 & 31 & 4 & 3 & 16 & 45 & 1 \\
\hline Third quintile group & 5,140 & 1,190 & 450 & 528 & 59 & 6 & 3 & 13 & 18 & 1 \\
\hline Fourth quintile group & 5,140 & 1,160 & 673 & 826 & 75 & 8 & 2 & 7 & 7 & 1 \\
\hline Highest twenty per cent & 5,140 & 1,130 & 1,359 & 1,757 & 77 & 12 & 5 & 3 & 2 & 0 \\
\hline
\end{tabular}

Note: Please see page xiii for symbols and conventions used in this report.

1 Other than social security benefits.

2 Excluding housing benefit and council tax benefit (rates rebate in Northern Ireland) - see definitions in Appendix B.

\section{Table A43}

Income and source of income by household tenure, 2008

based on weighted data

\begin{tabular}{|c|c|c|c|c|c|c|c|c|c|c|}
\hline & \multirow{2}{*}{$\begin{array}{c}\text { Weighted } \\
\text { number } \\
\text { of house- } \\
\text { holds }\end{array}$} & \multirow{2}{*}{$\begin{array}{r}\text { Number } \\
\text { of house- } \\
\text { holds } \\
\text { in the } \\
\text { sample }\end{array}$} & \multicolumn{2}{|c|}{$\begin{array}{c}\text { Weekly } \\
\text { household } \\
\text { income }\end{array}$} & \multicolumn{6}{|c|}{ Source of income } \\
\hline & & & $\begin{array}{c}\text { Dispo- } \\
\text { sable }\end{array}$ & Gross & $\begin{array}{r}\text { Wages } \\
\text { and } \\
\text { salaries }\end{array}$ & $\begin{array}{r}\text { Self } \\
\text { employ- } \\
\text { ment }\end{array}$ & $\begin{array}{l}\text { Invest- } \\
\text { ments }\end{array}$ & $\begin{array}{r}\text { Annuities } \\
\text { and } \\
\text { pensions }^{1}\end{array}$ & $\begin{array}{r}\text { Social } \\
\text { security } \\
\text { benefits }{ }^{2}\end{array}$ & $\begin{array}{l}\text { Other } \\
\text { sources }\end{array}$ \\
\hline Tenure of dwelling & (000s) & Number & $f$ & $f$ & & Perce & age of $\mathrm{g}$ & oss weekly & household & come \\
\hline \multicolumn{11}{|l|}{ Owners } \\
\hline Owned outright & 8,010 & 1,950 & 488 & 577 & 41 & 9 & 7 & 21 & 21 & 0 \\
\hline Buying with a mortgage ${ }^{3}$ & 9,900 & 2,230 & 788 & 998 & 80 & 11 & 2 & 2 & 4 & 1 \\
\hline All & 17,910 & 4,180 & 654 & 810 & 68 & 10 & 3 & 8 & 10 & 1 \\
\hline \multicolumn{11}{|l|}{ Social rented from } \\
\hline Council $^{4}$ & 2,720 & 600 & 255 & 281 & 43 & 4 & 0 & 4 & 48 & 1 \\
\hline Registered social landlord 5 & 2,010 & 440 & 314 & 354 & 51 & [2] & 0 & 5 & 41 & 1 \\
\hline All & 4,730 & 1,040 & 280 & 312 & 47 & 3 & 0 & 4 & 45 & 1 \\
\hline \multicolumn{11}{|l|}{ Private rented $^{6}$} \\
\hline Rent free & 340 & 70 & 356 & 418 & 65 & [5] & 2 & [5] & 21 & [2] \\
\hline Rent paid, unfurnished ${ }^{7}$ & 2,130 & 450 & 710 & 877 & 72 & 6 & 13 & 1 & 8 & 1 \\
\hline Rent paid, furnished & 580 & 110 & 469 & 568 & 80 & [5] & 1 & - & 5 & 10 \\
\hline All & 3,040 & 630 & 625 & 768 & 72 & 6 & 11 & 1 & 8 & 2 \\
\hline
\end{tabular}

Note: Please see page xiii for symbols and conventions used in this report.

1 Other than social security benefits.

2 Excluding housing benefit and council tax benefit (rates rebate in Northern Ireland) - see defintions in Appendix B.

3 Including shared owners (who own part of the equity and pay mortgage, part rent).

4 "Council" includes local authorities, new towns, and Scottish homes, but see note 5 below.

5 Formerly housing association.

6 All tenants whose accomodation goes with the job of someone in the household are allocated to "rented privately", even if the landlord is a local authority, housing association, or housing action trust, or if the accomodation is rent free. Squatters are also included in this category.

7 'Unfurnished' includes the answers: 'partly furnished'. 


\section{Table A44}

\section{Income and source of income by UK countries and}

\section{Government Office Regions, 2006-2008}

based on weighted data

\begin{tabular}{|c|c|c|c|c|c|c|c|c|c|c|}
\hline \multirow[b]{3}{*}{ Government Office Regions } & \multirow{3}{*}{$\begin{array}{r}\begin{array}{r}\text { Weighted } \\
\text { number } \\
\text { of house- } \\
\text { holds }\end{array} \\
(000 \mathrm{~s})\end{array}$} & \multirow{3}{*}{$\begin{array}{r}\begin{array}{r}\text { Total } \\
\text { number }\end{array} \\
\text { of house- } \\
\text { holds }\end{array}$} & \multicolumn{2}{|c|}{$\begin{array}{l}\text { Weekly } \\
\text { household } \\
\text { income }\end{array}$} & \multicolumn{6}{|c|}{ Source of income } \\
\hline & & & $\begin{array}{c}\text { Dispo- } \\
\text { sable }\end{array}$ & Gross & $\begin{array}{r}\text { Wages } \\
\text { and } \\
\text { salaries }\end{array}$ & $\begin{array}{r}\text { Self } \\
\text { employ- } \\
\text { ment }\end{array}$ & $\begin{array}{l}\text { Invest- } \\
\text { ments }\end{array}$ & $\begin{array}{r}\text { Annuities } \\
\text { and } \\
\text { pensions }{ }^{1}\end{array}$ & $\begin{array}{r}\text { Social } \\
\text { security } \\
\text { benefits }^{2}\end{array}$ & $\begin{array}{l}\text { Other } \\
\text { sources }\end{array}$ \\
\hline & & & $\mathrm{f}$ & $f$ & \multicolumn{6}{|c|}{ Percentage of gross weekly household income } \\
\hline United Kingdom & 25,490 & 18,630 & 544 & 669 & 67 & 9 & 4 & 7 & 13 & 1 \\
\hline North East & 1,130 & 770 & 449 & 538 & 68 & 6 & 2 & 6 & 17 & 1 \\
\hline North West & 2,930 & 1,890 & 482 & 587 & 66 & 7 & 2 & 8 & 16 & 1 \\
\hline Yorkshire and the Humber & 2,150 & 1,570 & 475 & 575 & 67 & 8 & 2 & 7 & 15 & 1 \\
\hline East Midlands & 1,900 & 1,360 & 496 & 610 & 66 & 7 & 5 & 7 & 14 & 1 \\
\hline West Midlands & 2,150 & 1,510 & 509 & 622 & 67 & 9 & 2 & 6 & 14 & 1 \\
\hline East & 2,310 & 1,670 & 579 & 712 & 68 & 10 & 3 & 8 & 11 & 1 \\
\hline London & 3,040 & 1,540 & 712 & 900 & 73 & 8 & 5 & 4 & 8 & 1 \\
\hline South East & 3,280 & 2,530 & 626 & 783 & 66 & 12 & 4 & 7 & 10 & 1 \\
\hline South West & 2,350 & 1,580 & 528 & 642 & 62 & 10 & 4 & 10 & 13 & 1 \\
\hline England & 21,240 & 14,440 & 554 & 683 & 67 & 9 & 4 & 7 & 12 & 1 \\
\hline Wales & 1,270 & 860 & 474 & 570 & 64 & 9 & 3 & 8 & 15 & 1 \\
\hline Scotland & 2,320 & 1,580 & 499 & 611 & 67 & 8 & 3 & 8 & 14 & 1 \\
\hline Northern Ireland & 650 & 1,760 & 512 & 616 & 66 & 10 & 2 & 6 & 15 & 1 \\
\hline
\end{tabular}

Note: Please see page xiii for symbols and conventions used in this report.

This table is based on a three year average.

1 Other than social security benefits.

2 Excluding housing benefit and council tax benefit (rates rebate in Northern Ireland) - see defintions in Appendix B.

\section{Table A45}

Income and source of income by GB urban/rural area, 2006-2008

based on weighted data

\begin{tabular}{|c|c|c|c|c|c|c|c|c|c|c|}
\hline & \multirow{2}{*}{$\begin{array}{r}\text { Weighted } \\
\text { number } \\
\text { of house- } \\
\text { holds }\end{array}$} & \multirow{2}{*}{$\begin{array}{r}\text { Total } \\
\text { number } \\
\text { of house- } \\
\text { holds }\end{array}$} & \multicolumn{2}{|c|}{$\begin{array}{c}\text { Weekly } \\
\text { household } \\
\text { income }\end{array}$} & \multicolumn{6}{|c|}{ Source of income } \\
\hline & & & $\begin{array}{c}\text { Dispo- } \\
\text { sable }\end{array}$ & Gross & $\begin{array}{r}\text { Wages } \\
\text { and } \\
\text { salaries }\end{array}$ & $\begin{array}{r}\text { Self } \\
\text { employ- } \\
\text { ment }\end{array}$ & $\begin{array}{l}\text { Invest- } \\
\text { ments }\end{array}$ & $\begin{array}{r}\text { Annuities } \\
\text { and } \\
\text { pensions }^{1}\end{array}$ & $\begin{array}{r}\text { Social } \\
\text { security } \\
\text { benefits }^{2}\end{array}$ & $\begin{array}{l}\text { Other } \\
\text { sources }\end{array}$ \\
\hline GB urban rural areas & (000s) & Number & $f$ & $f$ & \multicolumn{6}{|c|}{ Percentage of gross weekly household income } \\
\hline Urban & 19,540 & 13,070 & 532 & 655 & 69 & 8 & 3 & 6 & 13 & 1 \\
\hline Rural & 5,300 & 3,810 & 591 & 729 & 61 & 12 & 5 & 9 & 11 & 1 \\
\hline
\end{tabular}

Note: Please see page xiii for symbols and conventions used in this report.

This table is based on a three year average

1 Other than social security benefits.

2 Excluding housing benefit and council tax benefit (rates rebate in Northern Ireland) - see defintions in Appendix B. 
Table A46

Income and source of income by socio-economic classification, 2008

based on weighted data

\begin{tabular}{|c|c|c|c|c|c|c|c|c|c|c|}
\hline & \multirow{2}{*}{$\begin{array}{r}\text { Weighted } \\
\text { number } \\
\text { of house- } \\
\text { holds }\end{array}$} & \multirow{2}{*}{$\begin{array}{l}\text { Number } \\
\text { of house- } \\
\text { holds } \\
\text { in the } \\
\text { sample }\end{array}$} & \multicolumn{2}{|c|}{$\begin{array}{c}\text { Weekly } \\
\text { household } \\
\text { income }\end{array}$} & \multicolumn{6}{|c|}{ Source of income } \\
\hline & & & $\begin{array}{c}\text { Dispo- } \\
\text { sable }\end{array}$ & Gross & $\begin{array}{r}\text { Wages } \\
\text { and } \\
\text { salaries }\end{array}$ & $\begin{array}{r}\text { Self } \\
\text { employ- } \\
\text { ment }\end{array}$ & $\begin{array}{c}\text { Invest- } \\
\text { ments }\end{array}$ & $\begin{array}{r}\text { Annuities } \\
\text { and } \\
\text { pensions }^{1}\end{array}$ & $\begin{array}{r}\text { Social } \\
\text { security } \\
\text { benefits }\end{array}$ & $\begin{array}{l}\text { Other } \\
\text { sources }\end{array}$ \\
\hline NS-SEC Group ${ }^{3}$ & (000s) & Number & $f$ & $f$ & \multicolumn{6}{|c|}{ Percentage of gross weekly household income } \\
\hline Large employers/higher managerial & 1,190 & 260 & 1,502 & 1,985 & 85 & 1 & 11 & 1 & 1 & 0 \\
\hline Higher professional & 1,940 & 430 & 1,057 & 1,359 & 73 & 19 & 3 & 2 & 2 & 1 \\
\hline Lower managerial and professional & 4,590 & 1,040 & 783 & 1,011 & 86 & 6 & 2 & 3 & 3 & 1 \\
\hline Intermediate & 1,310 & 290 & 533 & 654 & 82 & 3 & 2 & 4 & 8 & 1 \\
\hline Small employers & 1,510 & 360 & 686 & 765 & 23 & 65 & 3 & 2 & 7 & 1 \\
\hline Lower supervisory & 1,790 & 390 & 615 & 766 & 89 & [2] & 1 & 2 & 6 & 1 \\
\hline Semi-routine & 1,930 & 420 & 464 & 554 & 81 & [2] & 1 & 3 & 12 & 1 \\
\hline Routine & 1,700 & 380 & 451 & 544 & 83 & [1] & 1 & 3 & 13 & 0 \\
\hline Long-term unemployed ${ }^{4}$ & 520 & 120 & 264 & 285 & 34 & [0] & 1 & [3] & 61 & [1] \\
\hline Students & 320 & 60 & 421 & 477 & 63 & [4] & [1] & {$[0]$} & 9 & 23 \\
\hline Occupation not stated 5 & 8,900 & 2,080 & 309 & 338 & 12 & 2 & 7 & 31 & 49 & 1 \\
\hline
\end{tabular}

Note: Please see page xiii for symbols and conventions used in this report.

1 Other than social security benefits.

2 Excluding housing benefit and council tax benefit (rates rebate in Northern Ireland) - see definitions in Appendix B.

3 National Statistics Socio-Economic Classification (NS-SEC) - see defintions in Appendix B.

4 Includes those who have never worked.

5 Includes those who are economically inactive - see defintions in Appendix B.

Table A47

Income and source of income, 1970 to 2008

\begin{tabular}{|c|c|c|c|c|c|c|c|c|c|c|c|c|}
\hline & \multirow{3}{*}{$\begin{array}{r}\text { Weighted } \\
\text { number } \\
\text { of house- } \\
\text { holds }\end{array}$} & \multirow{3}{*}{$\begin{array}{r}\text { Number } \\
\text { of house- } \\
\text { holds } \\
\text { in the } \\
\text { sample }\end{array}$} & \multicolumn{4}{|c|}{ Weekly household income ${ }^{1}$} & \multicolumn{6}{|c|}{ Source of income } \\
\hline & & & \multicolumn{2}{|c|}{ Current prices } & \multicolumn{2}{|c|}{ Constant prices } & \multirow{2}{*}{$\begin{array}{r}\text { Wages } \\
\text { and } \\
\text { salaries }\end{array}$} & \multirow{2}{*}{$\begin{array}{l}\text { Self } \\
\text { employ- } \\
\text { ment }\end{array}$} & \multirow{2}{*}{$\begin{array}{l}\text { Invest- } \\
\text { ments }\end{array}$} & \multirow{2}{*}{$\begin{array}{r}\text { Annuities } \\
\text { and } \\
\text { pensions }^{2}\end{array}$} & \multirow{2}{*}{$\begin{array}{r}\text { Social } \\
\text { security } \\
\text { benefits }^{3}\end{array}$} & \multirow{2}{*}{$\begin{array}{l}\text { Other } \\
\text { sources }\end{array}$} \\
\hline & & & $\begin{array}{c}\text { Dispo- } \\
\text { sable }\end{array}$ & Gross & $\begin{array}{c}\text { Dispo- } \\
\text { sable }\end{array}$ & Gross & & & & & & \\
\hline & (000s) & Number & $f$ & $f$ & $f$ & $f$ & & \multicolumn{5}{|c|}{ Percentage of gross weekly household income } \\
\hline 1970 & & 6,390 & 28 & 34 & 330 & 398 & 77 & 7 & 4 & 3 & 9 & 1 \\
\hline 1980 & & 6,940 & 115 & 140 & 369 & 451 & 75 & 6 & 3 & 3 & 13 & 1 \\
\hline 1990 & & 7,050 & 258 & 317 & 439 & 540 & 67 & 10 & 6 & 5 & 11 & 1 \\
\hline $1995-96$ & & 6,800 & 307 & 381 & 439 & 545 & 64 & 9 & 5 & 7 & 14 & 2 \\
\hline $1996-97$ & & 6,420 & 325 & 397 & 454 & 555 & 65 & 9 & 4 & 7 & 14 & 1 \\
\hline $1997-98$ & & 6,410 & 343 & 421 & 464 & 569 & 67 & 8 & 4 & 7 & 13 & 1 \\
\hline $1998-99^{4}$ & 24,660 & 6,630 & 371 & 457 & 487 & 599 & 68 & 8 & 4 & 7 & 12 & 1 \\
\hline $1999-2000$ & 25,340 & 7,100 & 391 & 480 & 505 & 620 & 66 & 10 & 5 & 7 & 12 & 1 \\
\hline $2001-02^{5}$ & 24,450 & 7,470 & 442 & 541 & 546 & 668 & 68 & 8 & 4 & 7 & 12 & 1 \\
\hline $2002-03$ & 24,350 & 6,930 & 453 & 552 & 549 & 668 & 68 & 8 & 3 & 7 & 12 & 1 \\
\hline 2003-04 & 24,670 & 7,050 & 464 & 570 & 546 & 671 & 67 & 9 & 3 & 7 & 13 & 1 \\
\hline 2004-05 & 24,430 & 6,800 & 489 & 601 & 558 & 686 & 68 & 8 & 3 & 7 & 13 & 1 \\
\hline 2005-06 & 24,800 & 6,790 & 500 & 616 & 557 & 685 & 67 & 8 & 3 & 7 & 13 & 1 \\
\hline $2006^{6}$ & 24,790 & 6,650 & 521 & 642 & 565 & 696 & 67 & 9 & 3 & 7 & 12 & 1 \\
\hline $2006^{7}$ & 25,440 & 6,650 & 515 & 635 & 559 & 688 & 67 & 9 & 3 & 7 & 13 & 1 \\
\hline 2007 & 25,350 & 6,140 & 534 & 659 & 555 & 686 & 67 & 8 & 4 & 7 & 13 & 1 \\
\hline 2008 & 25,690 & 5,850 & 582 & 713 & 582 & 713 & 67 & 9 & 4 & 7 & 12 & 1 \\
\hline
\end{tabular}

Note: Please see page xiii for symbols and conventions used in this report.

1 Does not include imputed income from owner-occupied and rent-free households.

2 Other than social security benefits.

3 Excluding housing benefit and council tax benefit (rates rebate in Northern Ireland) and their predecessors in earlier years - see Appendix B.

4 Based on weighted data from 1998-99.

5 From 2001-02 onwards, weighting is based on the population estimates from the 2001 Census.

6 From 1998-99 to this version of 2006, figures shown are based on weighted data using non-response weights based on the 1991 Census and population figures from the 1991 and 2001 Censuses.

7 From this version of 2006, figures shown are based on weighted data using updated weights, with non-response weights and population figures based on the 2001 Census. 


\section{Table A48}

Characteristics of households, 2008

based on weighted data

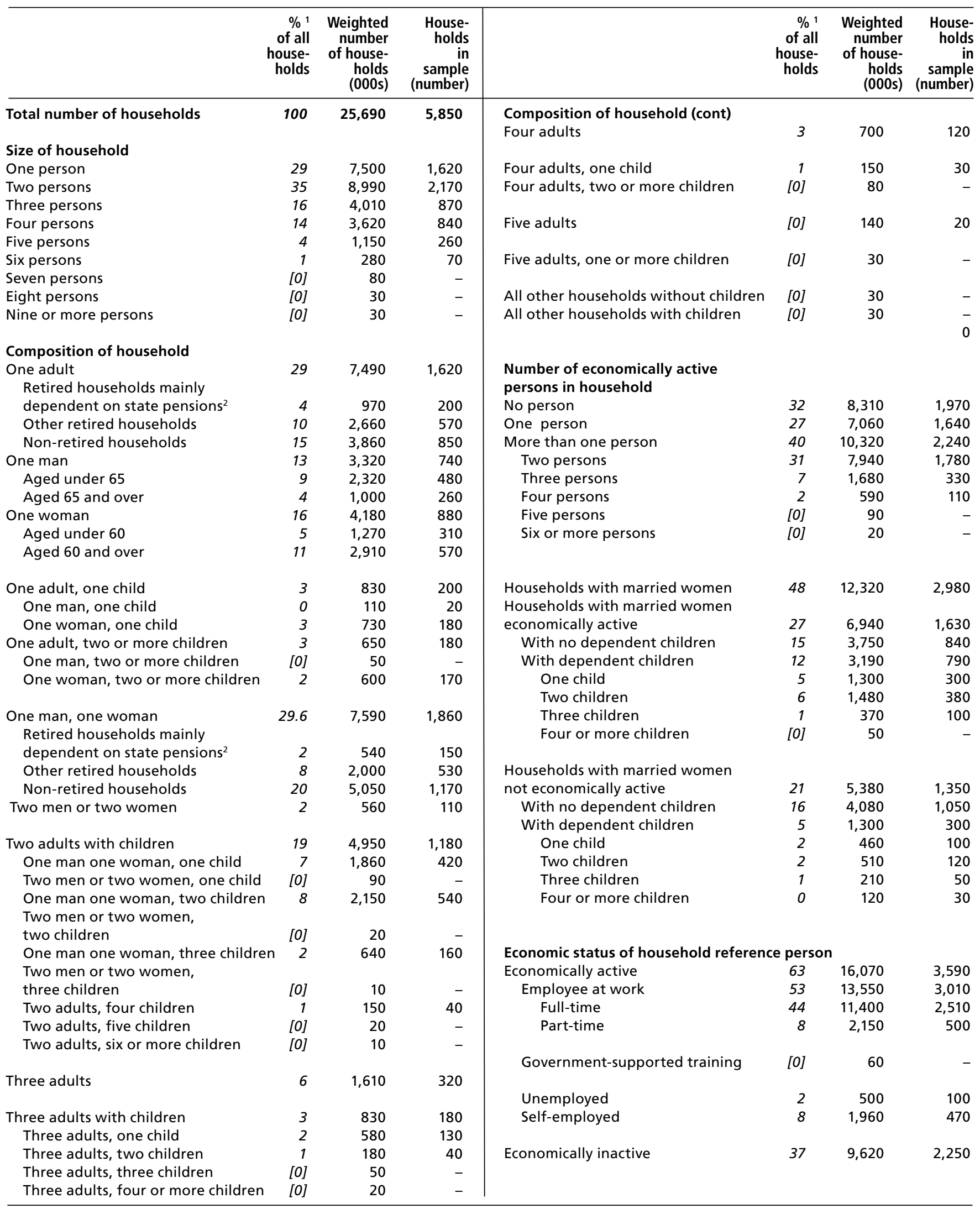

Note: Please see page xiii for symbols and conventions used in this report.

1 Based on weighted number of households.

2 Mainly dependent on state pensions and not economically active - see definitions in Appendix B. 
Table A48

Characteristics of households, 2008 (cont.)

based on weighted data

\begin{tabular}{|c|c|c|c|c|c|c|c|}
\hline & $\begin{array}{r}\% 1 \\
\text { of all } \\
\text { house- } \\
\text { holds }\end{array}$ & $\begin{array}{r}\text { Weighted } \\
\text { number } \\
\text { of house- } \\
\text { holds } \\
(000 \mathrm{~s})\end{array}$ & $\begin{array}{r}\text { House- } \\
\text { holds } \\
\text { in } \\
\text { sample } \\
\text { (number) }\end{array}$ & & $\begin{array}{r}\%^{1} \\
\text { of all } \\
\text { house- } \\
\text { holds }\end{array}$ & $\begin{array}{r}\text { Weighted } \\
\text { number } \\
\text { of house- } \\
\text { holds } \\
(000 \mathrm{~s})\end{array}$ & $\begin{array}{r}\text { House- } \\
\text { holds } \\
\text { in } \\
\text { sample } \\
\text { (number) }\end{array}$ \\
\hline Age of household reference person & & & & GB urban/rural areas (over 3 years) & & & \\
\hline 15 and under 20 years & [0] & 80 & - & GB Urban & 79 & 19,540 & 4,360 \\
\hline 20 and under 25 years & 3 & 810 & 130 & GB rural & 21 & 5,300 & 1,270 \\
\hline 25 and under 30 years & 6 & 1,640 & 310 & & & & \\
\hline 30 and under 35 years & 8 & 1,980 & 440 & & & & \\
\hline 35 and under 40 years & 10 & 2,520 & 570 & Tenure of dwelling 5 & & & \\
\hline \multirow[t]{2}{*}{40 and under 45 years } & 10 & 2,660 & 600 & Owners & & & \\
\hline & & & & Owned outright & 31 & 8,010 & 1,950 \\
\hline 45 and under 50 years & 10 & 2,580 & 620 & Buying with a mortgage & 39 & 9,900 & 2,230 \\
\hline 50 and under 55 years & 10 & 2,450 & 550 & All & 70 & 17,910 & 4,180 \\
\hline \multirow[t]{2}{*}{55 and under 60 years } & 8 & 2,010 & 490 & Social rented from & & & \\
\hline & & & & Council & 11 & 2,720 & 600 \\
\hline 60 and under 65 years & 9 & 2,290 & 550 & Registered social landlord & 8 & 2,010 & 440 \\
\hline 65 and under 70 years & 6 & 1,650 & 420 & All & 18 & 4,730 & 1,040 \\
\hline \multirow[t]{2}{*}{70 and under 75 years } & 6 & 1,490 & 390 & Private rented & & & \\
\hline & & & & Rent free & 1 & 340 & 70 \\
\hline 75 and under 80 years & 6 & 1,480 & 340 & Rent paid, unfurnished & 8 & 2,130 & 450 \\
\hline 80 and under 85 years & 4 & 1,080 & 240 & Rent paid, furnished & 2 & 580 & 110 \\
\hline 85 and under 90 years & 3 & 760 & 150 & All & 12 & 3,040 & 630 \\
\hline \multirow[t]{2}{*}{90 years or more } & 1 & 210 & 40 & & & & \\
\hline & & & & Households with durable goods & & & \\
\hline \multicolumn{4}{|l|}{ Government Office Regions and Countries } & Car/van & 74 & 19,140 & 4,500 \\
\hline \multirow[t]{2}{*}{ 2006-2008 (3 year average) } & & & & One & 43 & 11,070 & 2,620 \\
\hline & & & & Two & 25 & 6,450 & 1,540 \\
\hline United Kingdom & 100 & 25,490 & 6,210 & Three or more & 6 & 1,610 & 340 \\
\hline \multirow[t]{2}{*}{ North East } & 4 & 1,130 & 260 & Central heating, full or partial & 95 & 24,410 & 5,580 \\
\hline & & & & Fridge-freezer or deep freezer & 97 & 24,910 & 5,670 \\
\hline North West & 11 & 2,930 & 630 & Washing machine & 96 & 24,620 & 5,620 \\
\hline \multirow[t]{2}{*}{ Yorkshire and the Humber } & 8 & 2,150 & 520 & Tumble dryer & 59 & 15,100 & 3,520 \\
\hline & & & & Dishwasher & 37 & 9,630 & 2,330 \\
\hline East Midlands & 7 & 1,900 & 450 & Microwave oven & 92 & 23,650 & 5,400 \\
\hline West Midlands & 8 & 2,150 & 500 & & & & \\
\hline \multirow[t]{2}{*}{ East } & 9 & 2,310 & 560 & Telephone & 90 & 23,150 & 5,300 \\
\hline & & & & Mobile phone & 79 & 20,180 & 4,520 \\
\hline London & 12 & 3,040 & 510 & Video recorder & 70 & 17,980 & 4,160 \\
\hline South East & 13 & 3,280 & 840 & DVD Player & 88 & 22,510 & 5,110 \\
\hline \multirow[t]{2}{*}{ South West } & 9 & 2,350 & 530 & Satellite receiver ${ }^{6}$ & 82 & 21,170 & 4,850 \\
\hline & & & & Compact disc player & 86 & 22,140 & 5,070 \\
\hline England & 83 & 21,240 & 4,810 & Home computer & 72 & 18,520 & 4,190 \\
\hline Wales & 5 & 1,270 & 290 & Internet connection & 66 & 17,000 & 3,840 \\
\hline Scotland & 9 & 2,320 & 530 & & & & \\
\hline Northern Ireland & 3 & 650 & 590 & & & & \\
\hline \multicolumn{8}{|l|}{$\begin{array}{l}\text { Socio-economic classification } \\
\text { of household reference person }\end{array}$} \\
\hline Higher managerial and professional & 12 & 3,130 & 700 & & & & \\
\hline Large employers/higher managerial & 5 & 1,190 & 260 & & & & \\
\hline Higher professional & 8 & 1,940 & 430 & & & & \\
\hline Lower managerial and professional & 18 & 4,590 & 1,040 & & & & \\
\hline Intermediate & 5 & 1,310 & 290 & & & & \\
\hline Small employers & 6 & 1,510 & 360 & & & & \\
\hline Lower supervisory & 7 & 1,790 & 390 & & & & \\
\hline Semi-routine & 7 & 1,930 & 420 & & & & \\
\hline Routine & 7 & 1,700 & 380 & & & & \\
\hline Long-term unemployed ${ }^{3}$ & 2 & 520 & 120 & & & & \\
\hline Students & 1 & 320 & 60 & & & & \\
\hline Occupation not stated ${ }^{4}$ & 35 & 8,900 & 2,080 & & & & \\
\hline
\end{tabular}

Note: Please see page xiii for symbols and conventions used in this report.

1 Based on weighted number of households.

2 Mainly dependent on state pensions and not economically active - see definitions in Appendix B.

3 Includes those who have never worked.

4 Includes those who are economically inactive - see definitions in Appendix B.

5 See footnotes in Table A34.

6 Includes digital and cable receivers. 


\section{Table A49}

\section{Characteristics of persons, 2008}

based on weighted data

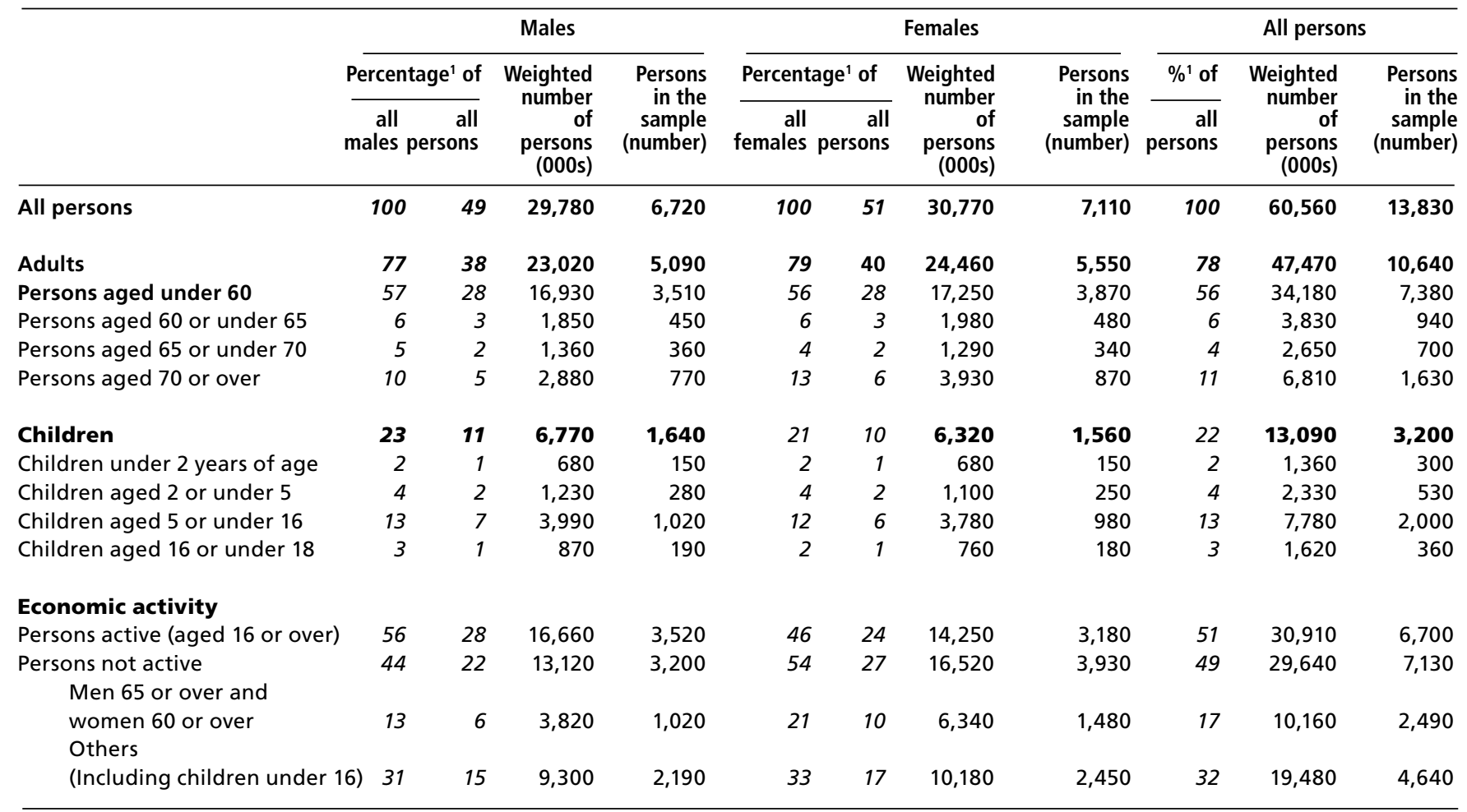

Note: Please see page xiii for symbols and conventions used in this report.

1 Based on weighted number of households. 


\section{Table A50}

\section{Percentage of households with durable goods, 1970 to 2008}

\begin{tabular}{|c|c|c|c|c|c|c|c|c|c|c|c|c|c|c|}
\hline & $\begin{array}{l}\text { Carl } \\
\text { van }\end{array}$ & $\begin{array}{r}\text { Central } \\
\text { heating }\end{array}$ & $\begin{array}{l}\text { Washing } \\
\text { machine }\end{array}$ & $\begin{array}{r}\text { Tumble } \\
\text { dryer }\end{array}$ & $\begin{array}{r}\text { Dish- } \\
\text { washer }\end{array}$ & $\begin{array}{l}\text { Micro- } \\
\text { wave }\end{array}$ & $\begin{array}{c}\text { Tele- } \\
\text { phone }\end{array}$ & $\begin{array}{c}\text { Mobile } \\
\text { phone }\end{array}$ & $\begin{array}{r}\text { Video } \\
\text { recorder }\end{array}$ & $\begin{array}{r}\text { DVD } \\
\text { Player }\end{array}$ & $\begin{array}{l}\text { Satellite } \\
\text { receiver }^{2}\end{array}$ & $\begin{array}{r}\text { Cd } \\
\text { player }\end{array}$ & $\begin{array}{c}\text { Home } \\
\text { computer }\end{array}$ & $\begin{array}{r}\text { Internet } \\
\text { connection }\end{array}$ \\
\hline 1970 & 52 & 30 & 65 & - & - & - & 35 & - & - & - & - & - & - & - \\
\hline 1975 & 57 & 47 & 72 & - & - & - & 52 & - & - & - & - & - & - & - \\
\hline 1980 & 60 & 59 & 79 & - & - & - & 72 & - & - & - & - & - & - & - \\
\hline 1985 & 63 & 69 & 83 & - & - & - & 81 & - & 30 & - & - & - & 13 & - \\
\hline 1990 & 67 & 79 & 86 & - & - & - & 87 & - & 61 & - & - & - & 17 & - \\
\hline 1994-95 & 69 & 84 & 89 & 50 & 18 & 67 & 91 & - & 76 & - & - & 46 & - & - \\
\hline $1995-96$ & 70 & 85 & 91 & 50 & 20 & 70 & 92 & - & 79 & - & - & 51 & - & - \\
\hline $1996-97$ & 69 & 87 & 91 & 51 & 20 & 75 & 93 & 16 & 82 & - & 19 & 59 & 27 & - \\
\hline $1997-98$ & 70 & 89 & 91 & 51 & 22 & 77 & 94 & 20 & 84 & - & 26 & 63 & 29 & - \\
\hline 1998-99 & 72 & 89 & 92 & 51 & 24 & 80 & 95 & 26 & 86 & - & 27 & 68 & 32 & 9 \\
\hline $1998-99^{3}$ & 72 & 89 & 92 & 51 & 23 & 79 & 95 & 27 & 85 & - & 28 & 68 & 33 & 10 \\
\hline 1999-2000 & 71 & 90 & 91 & 52 & 23 & 80 & 95 & 44 & 86 & - & 32 & 72 & 38 & 19 \\
\hline $2000-01$ & 72 & 91 & 92 & 53 & 25 & 84 & 93 & 47 & 87 & - & 40 & 77 & 44 & 32 \\
\hline $2001-02,{ }^{4}$ & 74 & 92 & 93 & 54 & 27 & 86 & 94 & 64 & 90 & - & 43 & 80 & 49 & 39 \\
\hline $2002-03$ & 74 & 93 & 94 & 56 & 29 & 87 & 94 & 70 & 90 & 31 & 45 & 83 & 55 & 45 \\
\hline 2003-04 & 75 & 94 & 94 & 57 & 31 & 89 & 92 & 76 & 90 & 50 & 49 & 86 & 58 & 49 \\
\hline 2004-05 & 75 & 95 & 95 & 58 & 33 & 90 & 93 & 78 & 88 & 67 & 58 & 87 & 62 & 53 \\
\hline 2005-06 & 74 & 94 & 95 & 58 & 35 & 91 & 92 & 79 & 86 & 79 & 65 & 88 & 65 & 55 \\
\hline $2006^{5}$ & 76 & 95 & 96 & 59 & 38 & 91 & 91 & 80 & 82 & 83 & 71 & 88 & 67 & 59 \\
\hline $2006^{6}$ & 74 & 95 & 96 & 59 & 37 & 91 & 91 & 79 & 82 & 83 & 70 & 87 & 67 & 58 \\
\hline 2007 & 75 & 95 & 96 & 57 & 37 & 91 & 89 & 78 & 75 & 86 & 77 & 86 & 70 & 61 \\
\hline 2008 & 74 & 95 & 96 & 59 & 37 & 92 & 90 & 79 & 70 & 88 & 82 & 86 & 72 & 66 \\
\hline
\end{tabular}

Note:

- Data not available.

1 Full or partial.

2 Includes digital and cable receivers.

3 From this version of 1998-99, figures shown are based on weighted data and including children's expenditure.

4 From 2001-02 onwards, weighting is based on the population figures from the 2001 census.

5 From 1998-99 to this version of 2006, figures shown are based on weighted data using non-response weights based on the 1991 Census and population figures from the 1991 and 2001 Censuses.

6 From this version of 2006, figures shown are based on weighted data using updated weights, with non-response weights and population figures based on the 2001 Census. 
Table A51

\title{
Percentage ${ }^{1}$ of households with durable goods by income group and household composition, 2008
}

based on weighted data

\begin{tabular}{|c|c|c|c|c|c|c|}
\hline & $\begin{array}{r}\text { Central } \\
\text { heating }^{2}\end{array}$ & $\begin{array}{l}\text { Washing } \\
\text { machine }\end{array}$ & $\begin{array}{r}\text { Tumble } \\
\text { dryer }\end{array}$ & $\begin{array}{l}\text { Micro- } \\
\text { wave }\end{array}$ & $\begin{array}{r}\text { Dish- } \\
\text { washer }\end{array}$ & $\begin{array}{r}\text { CD } \\
\text { player }\end{array}$ \\
\hline All households & 95 & 96 & 59 & 92 & 37 & 86 \\
\hline \multicolumn{7}{|c|}{ Gross income decile group } \\
\hline Lowest ten per cent & 93 & 85 & 37 & 86 & 11 & 70 \\
\hline Second decile group & 94 & 92 & 45 & 90 & 16 & 73 \\
\hline Third decile group & 93 & 92 & 49 & 92 & 18 & 79 \\
\hline Fourth decile group & 93 & 97 & 57 & 91 & 27 & 85 \\
\hline Fifth decile group & 94 & 98 & 58 & 93 & 33 & 90 \\
\hline Sixth decile group & 95 & 98 & 63 & 92 & 39 & 89 \\
\hline Seventh decile group & 96 & 99 & 65 & 95 & 42 & 93 \\
\hline Eighth decile group & 97 & 99 & 69 & 93 & 50 & 95 \\
\hline Ninth decile group & 97 & 100 & 70 & 95 & 61 & 93 \\
\hline Highest ten per cent & 99 & 100 & 75 & 92 & 77 & 96 \\
\hline
\end{tabular}

\section{Household composition}

One adult, retired households ${ }^{3}$

One adult, non-retired households

One adult, one child

One adult, two or more children

$93 \quad 86$

One man and one woman, retired households ${ }^{3}$

One man and one woman, non-retired households

One man and one woman, one child

One man and one woman, two or more children

\begin{tabular}{rrrrr}
86 & 39 & 85 & 12 & 57 \\
93 & 45 & 90 & 22 & 83 \\
99 & 63 & 94 & 27 & 88 \\
100 & 66 & 91 & 26 & 90 \\
& & & & \\
97 & 51 & 91 & 23 & 81 \\
99 & 64 & 93 & 46 & 92 \\
100 & 66 & 94 & 45 & 90 \\
99 & 75 & 95 & 60 & 91 \\
99 & 66 & 94 & 45 & 93 \\
96 & 67 & 98 & 43 & 91 \\
\hline rnet & Tele- & $\begin{array}{r}\text { Mobile } \\
\text { phone }\end{array}$ & $\begin{array}{c}\text { Satellite } \\
\text { receiver }\end{array}$ & $\begin{array}{r}\text { DVD } \\
\text { Phoner }\end{array}$ \\
\hline 66 & 90 & 79 & 82 & 88
\end{tabular}

\begin{tabular}{lrrrrrr} 
All other households without children & 96 & 99 & 66 & 94 & 95 & 93 \\
All other households with children & 99 & 96 & 97 & 98 \\
\hline & $\begin{array}{r}\text { Home } \\
\text { computer }\end{array}$ & $\begin{array}{r}\text { Internet } \\
\text { connection }\end{array}$ & $\begin{array}{r}\text { Tele- } \\
\text { phone }\end{array}$ & $\begin{array}{r}\text { Mobile } \\
\text { phone }\end{array}$ & $\begin{array}{r}\text { Satellite } \\
\text { receiver }\end{array}$ & $\begin{array}{r}\text { DVD } \\
\text { Player }\end{array}$ \\
\hline All households & $\mathbf{7 2}$ & $\mathbf{6 6}$ & $\mathbf{9 0}$ & $\mathbf{7 9}$ & $\mathbf{8 2}$ & $\mathbf{8 8}$ \\
\hline
\end{tabular}

\section{Gross income decile group}

Lowest ten per cent

Second decile group

Third decile group

Fourth decile group

Fifth decile group

\begin{tabular}{llllll}
33 & 26 & 74 & 61 & 63 & 66 \\
41 & 33 & 87 & 62 & 73 & 73 \\
47 & 40 & 88 & 66 & 74 & 78 \\
65 & 54 & 87 & 77 & 82 & 87 \\
75 & 68 & 91 & 83 & 86 & 91 \\
& & & & & \\
83 & 76 & 90 & 86 & 85 & 93 \\
91 & 85 & 94 & 87 & 88 & 95 \\
93 & 88 & 95 & 86 & 91 & 97 \\
95 & 94 & 97 & 89 & 91 & 97 \\
98 & 96 & 98 & 88 & 91 & 98 \\
\hline
\end{tabular}

Sixth decile group

Seventh decile group

Eighth decile group

Ninth decile group

Highest ten per cent

prop

\section{Household composition}

One adult, retired households ${ }^{3}$

One adult, non-retired households

One adult, one child

One adult, two or more children

\begin{tabular}{llllll}
15 & 10 & 96 & 34 & 53 & 48 \\
67 & 58 & 79 & 82 & 76 & 85 \\
73 & 61 & 68 & 90 & 86 & 97 \\
77 & 65 & 69 & 82 & 89 & 95 \\
35 & & & & & \\
87 & 29 & 97 & 63 & 81 & 81 \\
91 & 81 & 94 & 88 & 88 & 96 \\
93 & 84 & 90 & 86 & 91 & 97 \\
89 & 91 & 92 & 86 & 92 & 97 \\
85 & 86 & 91 & 85 & 84 & 97 \\
& 82 & 84 & 91 & 92 & 98 \\
\hline
\end{tabular}

One man and one woman, retired households ${ }^{3}$

One man and one woman, non-retired households

One man and one woman, one child

One man and one woman, two or more children

All other households without children

All other households with children

93
96
96

CD

(1)

Note: Please see page xiii for symbols and conventions used in this report..

1 See table A52 for number of recording households.

2 Full or partial.

3 Mainly dependent on state pensions and not economically active - see Appendix B.

4 Includes digital and cable receivers. 
Table A52

\section{Percentage of households with cars by income group, tenure and household} composition, 2008

based on weighted data

\begin{tabular}{|c|c|c|c|c|c|c|}
\hline & $\begin{array}{r}\text { One } \\
\text { car/van }\end{array}$ & $\begin{array}{r}\text { Two } \\
\text { cars/vans }\end{array}$ & $\begin{array}{r}\text { Three } \\
\text { or more } \\
\text { cars/vans }\end{array}$ & $\begin{array}{l}\text { All with } \\
\text { cars/vans }\end{array}$ & $\begin{array}{r}\text { Weighted } \\
\text { number } \\
\text { of house- } \\
\text { holds } \\
(000 s)\end{array}$ & $\begin{array}{r}\text { House- } \\
\text { holds } \\
\text { in the } \\
\text { sample } \\
\text { (number) }\end{array}$ \\
\hline All households & 43 & 25 & 6 & 74 & 25,690 & 5,850 \\
\hline \multicolumn{7}{|l|}{ Gross income decile group } \\
\hline Lowest ten per cent & 26 & [2] & {$[0]$} & 29 & 2,570 & 550 \\
\hline Second decile group & 38 & 4 & [1] & 44 & 2,570 & 580 \\
\hline Third decile group & 49 & 5 & [0] & 55 & 2,570 & 610 \\
\hline Fourth decile group & 62 & 11 & [1] & 74 & 2,570 & 630 \\
\hline Fifth decile group & 63 & 18 & [3] & 84 & 2,570 & 590 \\
\hline Sixth decile group & 53 & 28 & 4 & 85 & 2,570 & 600 \\
\hline Seventh decile group & 48 & 35 & 8 & 91 & 2,570 & 590 \\
\hline Eighth decile group & 41 & 43 & 8 & 93 & 2,560 & 570 \\
\hline Ninth decile group & 31 & 50 & 14 & 94 & 2,570 & 550 \\
\hline Highest ten per cent & 19 & 53 & 23 & 96 & 2,560 & 580 \\
\hline \multicolumn{7}{|l|}{ Tenure of dwelling ${ }^{1}$} \\
\hline \multicolumn{7}{|l|}{ Owners } \\
\hline Owned outright & 50 & 21 & 6 & 77 & 8,010 & 1,950 \\
\hline Buying with a mortgage & 41 & 41 & 9 & 92 & 9,900 & 2,230 \\
\hline All & 45 & 32 & 8 & 85 & 17,910 & 4,180 \\
\hline \multicolumn{7}{|l|}{ Social rented from } \\
\hline Council & 30 & 5 & [1] & 35 & 2,720 & 600 \\
\hline Registered social landlord ${ }^{2}$ & 37 & 7 & [4] & 48 & 2,010 & 440 \\
\hline All & 33 & 6 & [2] & 41 & 4,730 & 1,040 \\
\hline \multicolumn{7}{|l|}{ Private rented } \\
\hline Rent free & 39 & [15] & [4] & 59 & 340 & 70 \\
\hline Rent paid, unfurnished & 49 & 16 & [3] & 67 & 2,130 & 450 \\
\hline Rent paid, furnished & 40 & [11] & [1] & 51 & 580 & 110 \\
\hline All & 46 & 15 & [2] & 63 & 3,040 & 630 \\
\hline \multicolumn{7}{|l|}{ Household composition } \\
\hline One adult, retired mainly dependent on state pensions ${ }^{3}$ & 28 & [0] & - & 28 & 970 & 200 \\
\hline One adult, other retired & 41 & [0] & [0] & 41 & 2,660 & 570 \\
\hline One adult, non-retired & 57 & 6 & [1] & 64 & 3,860 & 850 \\
\hline One adult, one child & 54 & [2] & [0] & 56 & 830 & 200 \\
\hline One adult, two or more children & 46 & [1] & - & 47 & 650 & 180 \\
\hline $\begin{array}{l}\text { One man and one woman, retired mainly } \\
\text { dependent on state pensions }{ }^{3}\end{array}$ & 71 & [5] & [1] & 76 & 540 & 150 \\
\hline One man and one woman, other retired & 65 & 17 & [2] & 84 & 2,000 & 530 \\
\hline One man and one woman, non-retired & 41 & 44 & 5 & 89 & 5,050 & 1,170 \\
\hline One man and one woman, one child & 39 & 43 & 5 & 87 & 1,860 & 420 \\
\hline One man and one woman, two children & 36 & 51 & 5 & 91 & 2,150 & 540 \\
\hline One man and one woman, three children & 31 & 50 & [6] & 87 & 640 & 160 \\
\hline Two adults, four or more children & [22] & 43 & [7] & 72 & 180 & 50 \\
\hline Three adults & 31 & 34 & 24 & 89 & 1,610 & 320 \\
\hline Three adults, one or more children & 29 & 35 & 21 & 86 & 830 & 180 \\
\hline All other households without children & 27 & 26 & 27 & 80 & 1,440 & 260 \\
\hline All other households with children & 33 & {$[20]$} & 27 & 80 & 410 & 80 \\
\hline
\end{tabular}

Note: Please see page xiii for symbols and conventions used in this report.

1 See footnotes in Table A34.

2 Formerly housing association.

3 Mainly dependent on state pensions and not economically active - see Appendix B. 


\section{Table A53}

Percentage of households with durable goods by UK countries and Government Office Regions, 2006-2008

based on weighted data

\begin{tabular}{|c|c|c|c|c|c|c|c|}
\hline & $\begin{array}{r}\text { North } \\
\text { East }\end{array}$ & $\begin{array}{l}\text { North } \\
\text { West }\end{array}$ & $\begin{array}{l}\text { Yorks } \\
\text { \& the } \\
\text { Humber }\end{array}$ & $\begin{array}{r}\text { East } \\
\text { Midlands }\end{array}$ & $\begin{array}{r}\text { West } \\
\text { Midlands }\end{array}$ & East & London \\
\hline Average weighted number of households (thousands) & 1,130 & 2,930 & 2,150 & 1,900 & 2,150 & 2,310 & 3,040 \\
\hline Total number of households in sample (over 3 years) & 770 & 1,890 & 1,570 & 1,360 & 1,510 & 1,670 & 1,540 \\
\hline \multicolumn{8}{|l|}{$\begin{array}{l}\text { Percentage of households } \\
\text { by Government Office Region and country }\end{array}$} \\
\hline Car/van & 66 & 71 & 73 & 79 & 75 & 83 & 63 \\
\hline One & 41 & 42 & 45 & 45 & 43 & 45 & 44 \\
\hline Two & 20 & 25 & 24 & 28 & 25 & 30 & 16 \\
\hline Three or more & 4 & 5 & 5 & 7 & 7 & 8 & 3 \\
\hline Central heating full or partial & 98 & 94 & 94 & 96 & 94 & 96 & 95 \\
\hline Fridge-freezer or deep freezer & 96 & 96 & 96 & 97 & 95 & 96 & 94 \\
\hline Washing machine & 98 & 97 & 96 & 97 & 97 & 97 & 96 \\
\hline Tumble dryer & 55 & 59 & 59 & 59 & 63 & 61 & 45 \\
\hline Dishwasher & 25 & 32 & 32 & 37 & 32 & 43 & 37 \\
\hline Microwave & 92 & 94 & 94 & 93 & 93 & 91 & 87 \\
\hline Telephone & 88 & 88 & 88 & 91 & 89 & 93 & 90 \\
\hline Mobile phone & 73 & 78 & 81 & 84 & 84 & 83 & 79 \\
\hline DVD player & 87 & 88 & 85 & 86 & 86 & 86 & 83 \\
\hline Satellite receiver ${ }^{1}$ & 79 & 83 & 77 & 76 & 75 & 77 & 71 \\
\hline CD player & 86 & 87 & 86 & 88 & 87 & 89 & 82 \\
\hline Home computer & 64 & 68 & 66 & 70 & 69 & 71 & 73 \\
\hline \multirow[t]{2}{*}{ Internet connection } & 57 & 59 & 58 & 62 & 61 & 65 & 66 \\
\hline & $\begin{array}{l}\text { South } \\
\text { East }\end{array}$ & $\begin{array}{l}\text { South } \\
\text { West }\end{array}$ & England & Wales & Scotland & $\begin{array}{l}\text { Northern } \\
\text { Ireland }\end{array}$ & $\begin{array}{r}\text { United } \\
\text { Kingdom }\end{array}$ \\
\hline Weighted number of households (thousands) & 3,280 & 2,350 & 21,240 & 1,270 & 2,320 & 650 & 25,490 \\
\hline Total number of households in sample (over 3 years) & 2,530 & 1,580 & 14,440 & 860 & 1,580 & 1,760 & 18,630 \\
\hline
\end{tabular}

Percentage of households

by Government Office Region and country

Car/van

One

Two

Three or more

Central heating full or partial

Fridge-freezer or deep freezer

Washing machine

Tumble dryer

Dishwasher

Microwave

Telephone

Mobile phone

DVD Player

Satellite receiver ${ }^{1}$

CD player

Home computer

Internet connection

$\begin{array}{rrrrrrr}80 & 83 & 75 & 76 & 70 & 77 & 75 \\ 41 & 46 & 43 & 44 & 44 & 44 & 44 \\ 30 & 29 & 25 & 26 & 22 & 26 & 25 \\ 8 & 8 & 6 & 6 & 4 & 7 & 6 \\ 95 & 94 & 95 & 94 & 95 & 98 & 95 \\ 96 & 96 & 96 & 96 & 97 & 98 & 96 \\ 97 & 96 & 97 & 97 & 96 & 96 & 97 \\ 59 & 62 & 58 & 60 & 60 & 61 & 58 \\ 45 & 41 & 37 & 34 & 38 & 47 & 37 \\ 89 & 92 & 91 & 94 & 91 & 92 & 92 \\ 92 & 93 & 90 & 90 & 91 & 87 & 90 \\ 79 & 84 & 81 & 51 & 83 & 54 & 79 \\ 87 & 84 & & & & & \\ 76 & 76 & 86 & 83 & 87 & 81 & 86 \\ 88 & 88 & 76 & 77 & 79 & 78 & 77 \\ 74 & 70 & 87 & 86 & 87 & 81 & 87 \\ 68 & 63 & 70 & 68 & 68 & 64 & 70 \\ & & 63 & 59 & 60 & 55 & 62\end{array}$

Note: This table is based on a three year average.

1 Includes digital and cable receivers. 


\section{Table A54}

\section{Percentage of households by size, composition and age in each gross income decile group, 2008}

based on weighted data

\begin{tabular}{|c|c|c|c|c|c|c|}
\hline & $\begin{array}{r}\text { Lowest } \\
\text { ten } \\
\text { per cent }\end{array}$ & $\begin{array}{l}\text { Second } \\
\text { decile } \\
\text { group }\end{array}$ & $\begin{array}{l}\text { Third } \\
\text { decile } \\
\text { group }\end{array}$ & $\begin{array}{l}\text { Fourth } \\
\text { decile } \\
\text { group }\end{array}$ & $\begin{array}{l}\text { Fifth } \\
\text { decile } \\
\text { group }\end{array}$ & $\begin{array}{l}\text { Sixth } \\
\text { decile } \\
\text { group }\end{array}$ \\
\hline Lower boundary of group ( $\mathrm{f}$ per week) & & 146 & 224 & 305 & 408 & 522 \\
\hline Weighted number of households (thousands) & 2,570 & 2,570 & 2,570 & 2,570 & 2,570 & 2,570 \\
\hline Number of households in the sample & 550 & 580 & 610 & 630 & 590 & 600 \\
\hline \multicolumn{7}{|l|}{ Size of household } \\
\hline One person & 80 & 58 & 44 & 32 & 26 & U \\
\hline Two persons & 13 & 25 & 42 & 45 & 40 & 41 \\
\hline Three persons & 4 & 10 & 6 & 12 & 16 & 18 \\
\hline Four persons & [2] & 5 & 4 & 7 & 12 & 14 \\
\hline Five persons & [1] & [1] & [2] & [3] & 5 & 4 \\
\hline Six or more persons & - & [0] & [1] & [1] & [1] & [2] \\
\hline All sizes & 100 & 100 & 100 & 100 & 100 & 100 \\
\hline
\end{tabular}

Household composition

One adult, retired mainly

dependent on state pensions ${ }^{1}$

One adult, other retired

One adult, non-retired

One adult, one child

One adult, two or more children

$\begin{array}{rr}20 & 1 \\ 25 & 3 \\ 34 & 13 \\ 8 & \\ {[2]} & \end{array}$

$\begin{array}{rrrrr}12 & 5 & {[0]} & - & - \\ 33 & 22 & 11 & 8 & {[2]} \\ 13 & 17 & 20 & 19 & 18 \\ 5 & 6 & 6 & {[2]} & {[3]} \\ 7 & 5 & 5 & 3 & {[1]}\end{array}$

One man and one woman, retired

mainly dependent on state pensions ${ }^{1}$

One man and one woman, other retired

[0] 8

One man and one woman, non-retired

One man and one woman, one child

One man and one woman, two children

One man and one woman, three children

Two adults, four or more children

[2] 4

[1]

[0]

[2]

$[0]$

[0]

Three adults

Three adults, one or more children

[0] [0]

[0] [4]

$[1]$

$\left[\begin{array}{ll}{[0]} & {[1]} \\ {[4]} & {[1]}\end{array}\right.$

5

[1]

All other households without children

All other households with children

All compositions

$\begin{array}{rr}{[2]} & {[1]} \\ {[0]} & {[1]} \\ 100 & 100\end{array}$

[1] [2]

[1] [1]

$100 \quad 100$

$\begin{array}{rrr}3 & {[1]} & - \\ 20 & 14 & 9 \\ 14 & 20 & 25 \\ & & \\ 5 & 8 & 8 \\ 5 & 7 & 10 \\ {[2]} & {[3]} & {[3]} \\ {[1]} & {[0]} & {[1]} \\ & & \\ {[1]} & 5 & 8 \\ {[1]} & {[3]} & 5 \\ & & \\ {[2]} & 5 & 5 \\ {[2]} & {[2]} & {[1]} \\ 100 & 100 & 100\end{array}$

\section{Age of household reference person}

15 and under 20 years

20 and under 25 years

25 and under 30 years

30 and under 35 years

35 and under 40 years

40 and under 45 years

45 and under 50 years

50 and under 55 years

55 and under 60 years

60 and under 65 years

[0]

65 and under 70 years 70 and under 75 years

75 and under 80 years

80 and under 85 years

85 and under 90 years

90 years or more

All ages

$\begin{array}{rr}{[1]} & {[0]} \\ 6 & \\ 5 & \\ {[3]} & \\ 6 & \\ & \\ 7 & \\ 8 & \\ 6 & \\ 8 & 13] \\ 7 & 1 \\ 8 & 16 \\ 7 & 11 \\ 8 & \\ 10 & \\ 8 & 10 \\ {[2]} & \\ 100 & \end{array}$

Note: Please see page xiii for symbols and conventions used in this report

1 Mainly dependent on state pensions and not economically active - see Appendix B. 


\section{Table A54}

\section{Percentage of households by size, composition and age in each gross income decile group, 2008 (cont.)}

based on weighted data

\begin{tabular}{|c|c|c|c|c|c|}
\hline & $\begin{array}{r}\text { Seventh } \\
\text { decile } \\
\text { group }\end{array}$ & $\begin{array}{l}\text { Eighth } \\
\text { decile } \\
\text { group }\end{array}$ & $\begin{array}{l}\text { Ninth } \\
\text { decile } \\
\text { group }\end{array}$ & $\begin{array}{r}\text { Highest } \\
\text { ten } \\
\text { per cent }\end{array}$ & $\begin{array}{r}\text { All } \\
\text { house- } \\
\text { holds }\end{array}$ \\
\hline Lower boundary of group (f per week) & 664 & 817 & 1026 & 1356 & \\
\hline Weighted number of households (thousands) & 2,570 & 2,560 & 2,570 & 2,560 & 25,690 \\
\hline Number of households in the sample & 590 & 570 & 550 & 580 & 5,850 \\
\hline \multicolumn{6}{|l|}{ Size of household } \\
\hline One person & 15 & 8 & 6 & [3] & 29 \\
\hline Two persons & 34 & 40 & 35 & 34 & 35 \\
\hline Three persons & 21 & 25 & 23 & 21 & 16 \\
\hline Four persons & 23 & 19 & 24 & 30 & 14 \\
\hline Five persons & 6 & 7 & 8 & 8 & 4 \\
\hline Six or more persons & [2] & [2] & [3] & 4 & 2 \\
\hline All sizes & 100 & 100 & 100 & 100 & 100 \\
\hline
\end{tabular}

Household composition

One adult, retired mainly

dependent on state pensions ${ }^{1}$

One adult, other retired

One adult, non-retired

One adult, one child

One adult, two or more children

$\begin{array}{rrrrr}- & - & - & - & 4 \\ {[2]} & {[0]} & - & - & 10 \\ 13 & 7 & 6 & {[3]} & 15 \\ {[1]} & {[1]} & {[0]} & {[0]} & 3 \\ {[1]} & {[1]} & {[0]} & {[0]} & 3 \\ & & & & \\ - & - & - & - & 2 \\ 5 & 4 & {[2]} & {[2]} & 8 \\ 26 & 33 & 29 & 31 & 20 \\ & & & & \\ 11 & 12 & 10 & 10 & 7 \\ 16 & 11 & 14 & 16 & 8 \\ {[3]} & 4 & 3 & 5 & 2 \\ {[1]} & {[0]} & {[1]} & {[1]} & 1 \\ 8 & 12 & 13 & 11 & 6 \\ 5 & 4 & 7 & 6 & 3 \\ 7 & 8 & 11 & 13 & 6 \\ {[1]} & {[2]} & 4 & {[2]} & 2 \\ 100 & 100 & 100 & 100 & 100 \\ & & & & \end{array}$

One man and one woman, retired

mainly dependent on state pensions ${ }^{1}$

One man and one woman, other retired

One man and one woman, non-retired

One man and one woman, one child

One man and one woman, two children

One man and one woman, three children

Two adults, four or more children

Three adults

Three adults, one or more children

All other households without children

All other households with children

All compositions

100

Age of household reference person

15 and under 20 years

20 and under 25 years

25 and under 30 years

30 and under 35 years

35 and under 40 years

$\begin{array}{rrrrr}{[1]} & {[0]} & - & - & {[0]} \\ {[2]} & {[3]} & {[1]} & {[1]} & 3 \\ 9 & 9 & 10 & {[3]} & 6 \\ 11 & 12 & 9 & 9 & 8 \\ 14 & 13 & 15 & 15 & 10 \\ & & & & \\ 15 & 14 & 15 & 14 & 10 \\ 11 & 12 & 17 & 18 & 10 \\ 10 & 13 & 13 & 18 & 10 \\ 8 & 7 & 8 & 12 & 8 \\ 8 & 8 & 7 & 7 & 9 \\ & & & & \\ 4 & 5 & {[3]} & {[2]} & 6 \\ {[3]} & {[2]} & {[1]} & {[0]} & 6 \\ {[2]} & {[0]} & {[1]} & {[1]} & 6 \\ {[1]} & {[1]} & {[0]} & - & 4 \\ {[0]} & {[0]} & {[0]} & {[0]} & 3 \\ {[0]} & - & {[0]} & {[0]} & 1 \\ 100 & 100 & 100 & 100 & 100 \\ & & & & \end{array}$

40 and under 45 years

45 and under 50 years

50 and under 55 years

55 and under 60 years

60 and under 65 years

65 and under 70 years

70 and under 75 years

75 and under 80 years

80 and under 85 years

85 and under 90 years

90 years or more

All ages

100

Note: Please see page xiii for symbols and conventions used in this report.

1 Mainly dependent on state pensions and not economically active - see Appendix B. 


\section{Table A55}

\section{Percentage of households by economic activity, tenure and socio-economic} classification in each gross income decile group, 2008

based on weighted data

\begin{tabular}{|c|c|c|c|c|c|c|}
\hline & $\begin{array}{r}\text { Lowest } \\
\text { ten } \\
\text { per cent }\end{array}$ & $\begin{array}{l}\text { Second } \\
\text { decile } \\
\text { group }\end{array}$ & $\begin{array}{l}\text { Third } \\
\text { decile } \\
\text { group }\end{array}$ & $\begin{array}{l}\text { Fourth } \\
\text { decile } \\
\text { group }\end{array}$ & $\begin{array}{l}\text { Fifth } \\
\text { decile } \\
\text { group }\end{array}$ & $\begin{array}{r}\text { Sixth } \\
\text { decile } \\
\text { group }\end{array}$ \\
\hline Lower boundary of group ( $f$ per week) & & 146 & 224 & 305 & 408 & 522 \\
\hline Weighted number of households (thousands) & 2,570 & 2,570 & 2,570 & 2,570 & 2,570 & 2,570 \\
\hline Number of households in the sample & 550 & 580 & 610 & 630 & 590 & 600 \\
\hline \multicolumn{7}{|c|}{ Number of economically active persons in household } \\
\hline No person & 79 & 80 & 64 & 42 & 28 & 14 \\
\hline One person & 19 & 17 & 29 & 42 & 43 & 41 \\
\hline Two persons & [2] & 4 & 6 & 14 & 25 & 40 \\
\hline Three persons & - & - & - & [1] & [3] & 5 \\
\hline Four or more persons & - & - & - & {$[0]$} & [1] & [0] \\
\hline All economically active persons & 100 & 100 & 100 & 100 & 100 & 100 \\
\hline
\end{tabular}

Tenure of dwelling ${ }^{1}$

Owners

Owned outright

Buying with a mortgage

All

$28 \quad 46$

44

12

42

25

37

34

28

32

52

66

46

Social rented from

Council

Registered social landlord ${ }^{2}$

All

$\begin{array}{ll}36 & 21 \\ 15 & 16 \\ 50 & 37\end{array}$

$\begin{array}{ll}21 & 17 \\ 16 & 16 \\ 37 & 33\end{array}$

17
16
33

10

10

20

$\begin{array}{rr}8 & 5 \\ 6 & 7 \\ 15 & 12\end{array}$

Private rented

\section{Rent free}

Rent paid, unfurnished

Rent paid, furnished

All

All tenures

\begin{tabular}{rrrrrr}
4 & {$[1]$} & {$[1]$} & {$[1]$} & {$[2]$} & {$[1]$} \\
10 & 9 & 9 & 11 & 11 & 9 \\
4 & {$[1]$} & {$[2]$} & {$[2]$} & {$[1]$} & {$[4]$} \\
18 & 11 & 12 & 14 & 14 & 14 \\
100 & 100 & 100 & 100 & 100 & 100 \\
\hline
\end{tabular}

Socio-economic classification

Higher managerial and professional

Large employers/higher managerial

Higher professional

Lower managerial and professional

Intermediate

Small employers

Lower supervisory

Semi-routine

Routine

Long-term unemployed ${ }^{3}$

Students

Occupation not stated 4

All occupational groups

\begin{tabular}{rrrrrr}
- & {$[0]$} & {$[0]$} & {$[1]$} & {$[1]$} & {$[2]$} \\
{$[2]$} & {$[1]$} & {$[1]$} & {$[1]$} & {$[3]$} & 6 \\
{$[1]$} & {$[2]$} & 4 & 8 & 16 & 19 \\
{$[1]$} & {$[2]$} & 3 & 9 & 8 & 8 \\
{$[3]$} & {$[3]$} & 4 & 5 & 6 & 8 \\
{$[1]$} & {$[1]$} & {$[3]$} & 6 & 9 & 12 \\
5 & 3 & 10 & 11 & 11 & 12 \\
4 & 4 & 6 & 10 & 12 & 11 \\
8 & 4 & {$[2]$} & {$[2]$} & {$[2]$} & {$[1]$} \\
{$[3]$} & {$[2]$} & {$[1]$} & {$[1]$} & {$[1]$} & {$[1]$} \\
73 & 77 & 65 & 46 & 32 & 21 \\
100 & 100 & 100 & 100 & 100 & 100 \\
\hline
\end{tabular}

Note: Please see page xiii for symbols and conventions used in this report.

1 See footnotes in Table A34.

2 Formerly housing association.

3 Includes those who have never worked.

4 Includes those who are economically inactive - see definitions in Appendix B. 


\section{Table A55}

Percentage of households by economic activity, tenure and socio-economic classification in each gross income decile group, 2008 (cont.)

based on weighted data

\begin{tabular}{|c|c|c|c|c|c|}
\hline & $\begin{array}{r}\text { Seventh } \\
\text { decile } \\
\text { group }\end{array}$ & $\begin{array}{l}\text { Eighth } \\
\text { decile } \\
\text { group }\end{array}$ & $\begin{array}{l}\text { Ninth } \\
\text { decile } \\
\text { group }\end{array}$ & $\begin{array}{r}\text { Highest } \\
\text { ten } \\
\text { per cent }\end{array}$ & $\begin{array}{r}\text { All } \\
\text { house- } \\
\text { holds }\end{array}$ \\
\hline Lower boundary of group ( $f$ per week) & 664 & 817 & 1026 & 1356 & \\
\hline Weighted number of households (thousands) & 2,570 & 2,560 & 2,570 & 2,560 & 25,690 \\
\hline Number of households in the sample & 590 & 570 & 550 & 580 & 5,850 \\
\hline
\end{tabular}

Number of economically active persons in household

No person

One person

Two persons

Three persons

Four or more persons

All economically active persons

\begin{tabular}{rrrrr}
8 & 4 & {$[2]$} & {$[2]$} & 32 \\
31 & 21 & 15 & 15 & 27 \\
49 & 57 & 58 & 55 & 31 \\
9 & 14 & 16 & 18 & 7 \\
{$[3]$} & {$[4]$} & 8 & 11 & 3 \\
100 & 100 & 100 & 100 & 100 \\
\hline
\end{tabular}

Tenure of dwelling ${ }^{1}$

Owners

Owned outright

Buying with a mortgage

All

24

56

79
20

64

84

20

69

89

[2] [1]

[2]

5

All

Registered social landlord ${ }^{2}$

Private rented

Rent free

Rent paid, unfurnished

Rent paid, furnished

All

All tenures

Socio-economic classification

Higher managerial and professional

Large employers/higher managerial

Higher professional

Lower managerial and professional

Intermediate

Small employers

Lower supervisory

Semi-routine

Routine

Long-term unemployed ${ }^{3}$

Students

Occupation not stated ${ }^{4}$

All occupational groups

Note: Please see page xiii for symbols and conventions used in this report.

1 See footnotes in Table A34.

2 Formerly housing association.

3 Includes those who have never worked.

4 Includes those who are economically inactive - see definitions in Appendix B. 


\section{Table A56}

Average weekly household expenditure by OAC supergroup, 2008

based on weighted data and including children's expenditure

\begin{tabular}{|c|c|c|c|c|c|c|c|c|c|}
\hline & & $\begin{array}{r}\text { OAC } \\
\text { Super- } \\
\text { group } \\
1\end{array}$ & $\begin{array}{r}\text { OAC } \\
\text { Super- } \\
\text { group } \\
2\end{array}$ & $\begin{array}{r}\text { OAC } \\
\text { Super- } \\
\text { group } \\
3\end{array}$ & $\begin{array}{r}\text { OAC } \\
\text { Super- } \\
\text { group } \\
4\end{array}$ & $\begin{array}{r}\text { OAC } \\
\text { Super- } \\
\text { group } \\
5\end{array}$ & $\begin{array}{r}\text { OAC } \\
\text { Super- } \\
\text { group } \\
6\end{array}$ & $\begin{array}{r}\text { OAC } \\
\text { Super- } \\
\text { group } \\
7\end{array}$ & $\begin{array}{r}\text { All } \\
\text { house- } \\
\text { holds }\end{array}$ \\
\hline \multicolumn{2}{|r|}{ Weighted number of households (thousands) } & 4,180 & 1,520 & 3,450 & 5,480 & 3,290 & 4,890 & 2,890 & 25,690 \\
\hline \multicolumn{2}{|c|}{ Total number of households in sample } & 990 & 290 & 880 & 1,360 & 740 & 1,080 & 500 & 5,850 \\
\hline \multicolumn{2}{|c|}{ Total number of persons in sample } & 2,480 & 560 & 2,130 & 3,430 & 1,500 & 2,430 & 1,300 & 13,830 \\
\hline \multirow{2}{*}{\multicolumn{2}{|c|}{$\begin{array}{l}\text { Total number of adults in sample } \\
\text { Weighted average number of persons per household }\end{array}$}} & 1,800 & 480 & 1,650 & 2,680 & 1,170 & 1,930 & 930 & 10,640 \\
\hline & & 2.5 & 1.9 & 2.4 & 2.5 & 2.0 & 2.3 & 2.6 & 2.4 \\
\hline \multicolumn{3}{|c|}{ Commodity or service } & \multicolumn{7}{|c|}{ Average weekly household expenditure $(f)$} \\
\hline 1 & Food \& non-alcoholic drinks & 48.50 & 45.30 & 56.70 & 59.00 & 37.80 & 50.60 & 48.70 & 50.70 \\
\hline 2 & Alcoholic drinks, tobacco \& narcotics & 11.80 & 10.40 & 11.40 & 11.30 & 10.70 & 10.10 & 9.00 & 10.80 \\
\hline 3 & Clothing \& footwear & 17.60 & 24.40 & 22.00 & 27.20 & 15.20 & 21.50 & 22.10 & 21.60 \\
\hline 4 & Housing (net)', fuel \& power & 46.20 & 95.20 & 54.00 & 47.20 & 46.40 & 48.60 & 65.70 & 53.00 \\
\hline 5 & Household goods \& services & 23.60 & 29.80 & 36.80 & 42.80 & 17.20 & 30.80 & 21.20 & 30.10 \\
\hline 6 & Health & 4.80 & 5.90 & 5.70 & 6.50 & 3.20 & 5.10 & 4.30 & 5.10 \\
\hline 7 & Transport & 52.60 & 55.90 & 79.10 & 83.40 & 35.50 & 68.90 & 48.80 & 63.40 \\
\hline 8 & Communication & 11.30 & 13.10 & 12.10 & 12.50 & 10.50 & 12.20 & 12.40 & 12.00 \\
\hline 9 & Recreation \& culture & 50.50 & 54.20 & 76.80 & 87.60 & 38.70 & 55.30 & 37.20 & 60.10 \\
\hline 10 & Education & 2.20 & 19.70 & 11.00 & 7.70 & 2.00 & 3.50 & 5.80 & 6.20 \\
\hline 11 & Restaurants \& hotels & 31.20 & 48.70 & 41.90 & 47.70 & 22.60 & 38.30 & 33.70 & 37.70 \\
\hline 12 & Miscellaneous goods \& services & 27.20 & 48.60 & 41.50 & 47.10 & 20.90 & 36.00 & 28.50 & 35.60 \\
\hline $1-12$ & All expenditure groups & 327.50 & 451.40 & 448.90 & 479.90 & 260.80 & 380.70 & 337.50 & 386.30 \\
\hline
\end{tabular}

Note: Please see page xiii for symbols and conventions used in this report.

1 Excluding mortgage interest payments, council tax and Northern Ireland rates. 


\section{Table A57}

Average weekly household expenditure by OAC group, 2008

based on weighted data and including children's expenditure

\begin{tabular}{|c|c|c|c|c|c|c|c|c|c|c|c|c|}
\hline & & $\begin{array}{r}\text { OAC } \\
\text { group } \\
1 \mathrm{~A}\end{array}$ & $\begin{array}{r}\text { OAC } \\
\text { group } \\
1 \mathrm{~B}\end{array}$ & $\begin{array}{r}O A C \\
\text { group } \\
1 C\end{array}$ & $\begin{array}{r}O A C \\
\text { group } \\
2 A\end{array}$ & $\begin{array}{r}\text { OAC } \\
\text { group } \\
2 B\end{array}$ & $\begin{array}{r}O A C \\
\text { group } \\
3 A\end{array}$ & $\begin{array}{r}O A C \\
\text { group } \\
3 B\end{array}$ & $\begin{array}{r}O A C \\
\text { group } \\
3 C\end{array}$ & $\begin{array}{r}O A C \\
\text { group } \\
4 A\end{array}$ & $\begin{array}{r}O A C \\
\text { group } \\
4 B\end{array}$ & $\begin{array}{r}O A C \\
\text { group } \\
4 C\end{array}$ \\
\hline \multicolumn{2}{|r|}{ Weighted number of households (thousands) } & 1,090 & 1,760 & 1,340 & 560 & 960 & 1,430 & 960 & 1,050 & 1,140 & 1,540 & 1,790 \\
\hline \multicolumn{2}{|c|}{ Total number of households in sample } & 250 & 410 & 330 & 100 & 200 & 350 & 280 & 260 & 310 & 390 & 410 \\
\hline \multicolumn{2}{|c|}{ Total number of persons in sample } & 600 & 1,040 & 840 & 170 & 390 & 820 & 730 & 590 & 830 & 960 & 960 \\
\hline \multirow{2}{*}{\multicolumn{2}{|c|}{$\begin{array}{l}\text { Total number of adults in sample } \\
\text { Weighted average number of persons per household }\end{array}$}} & 460 & 720 & 620 & 160 & 330 & 630 & 560 & 460 & 610 & 770 & 780 \\
\hline & & 2.5 & 2.6 & 2.5 & 1.8 & 2.0 & 2.4 & 2.4 & 2.3 & 2.7 & 2.4 & 2.4 \\
\hline \multicolumn{4}{|c|}{ Commodity or service } & \multicolumn{9}{|c|}{ Average weekly household expenditure ( $\mathrm{f}$ ) } \\
\hline 1 & Food \& non-alcoholic drinks & 45.20 & 47.60 & 52.20 & 41.90 & 47.20 & 55.70 & 57.90 & 57.10 & 57.00 & 61.20 & 53.80 \\
\hline 2 & Alcoholic drinks, tobacco \& narcotics & 11.50 & 13.10 & 10.50 & 10.60 & 10.30 & 11.80 & 10.50 & 11.70 & 11.40 & 12.30 & 10.20 \\
\hline 3 & Clothing \& footwear & 17.10 & 18.30 & 16.90 & 30.80 & 20.70 & 18.70 & 26.10 & 22.50 & 35.90 & 28.00 & 21.40 \\
\hline 4 & Housing (net)', fuel \& power & 44.30 & 48.10 & 45.30 & 91.60 & 97.30 & 55.10 & 54.90 & 51.80 & 45.50 & 48.50 & 42.80 \\
\hline 5 & Household goods \& services & 18.00 & 21.00 & 31.60 & 25.70 & 32.30 & 31.50 & 38.70 & 42.40 & 44.30 & 42.10 & 36.10 \\
\hline 6 & Health & 3.50 & 6.50 & 3.80 & 4.40 & 6.80 & 3.90 & 5.60 & 8.20 & 4.50 & 6.40 & 7.10 \\
\hline 7 & Transport & 52.10 & 41.50 & 67.60 & 51.60 & 58.50 & 72.60 & 89.70 & 78.10 & 100.80 & 82.80 & 64.70 \\
\hline 8 & Communication & 10.20 & 11.80 & 11.60 & 13.30 & 12.90 & 11.70 & 12.50 & 12.20 & 14.30 & 11.90 & 11.00 \\
\hline 9 & Recreation \& culture & 54.80 & 43.70 & 55.90 & 50.60 & 56.30 & 62.60 & 92.00 & 82.30 & 94.90 & 116.20 & 63.80 \\
\hline 10 & Education & 1.20 & 3.10 & 1.70 & 10.20 & 25.20 & 10.00 & 13.60 & 9.90 & 12.40 & 8.60 & 2.60 \\
\hline 11 & Restaurants \& hotels & 25.80 & 33.20 & 33.10 & 45.40 & 50.70 & 35.80 & 38.80 & 53.00 & 54.80 & 50.40 & 37.90 \\
\hline 12 & Miscellaneous goods \& services & 23.80 & 25.50 & 32.30 & 39.80 & 53.80 & 40.10 & 39.30 & 45.40 & 54.00 & 45.00 & 42.70 \\
\hline
\end{tabular}

1-12 All expenditure groups

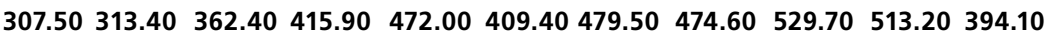

Note: Please see page xiii for symbols and conventions used in this report.

1 Excluding mortgage interest payments, council tax and Northern Ireland rates 


\section{Table A57}

Average weekly household expenditure by OAC group, 2008 (cont.)

based on weighted data and including children's expenditure

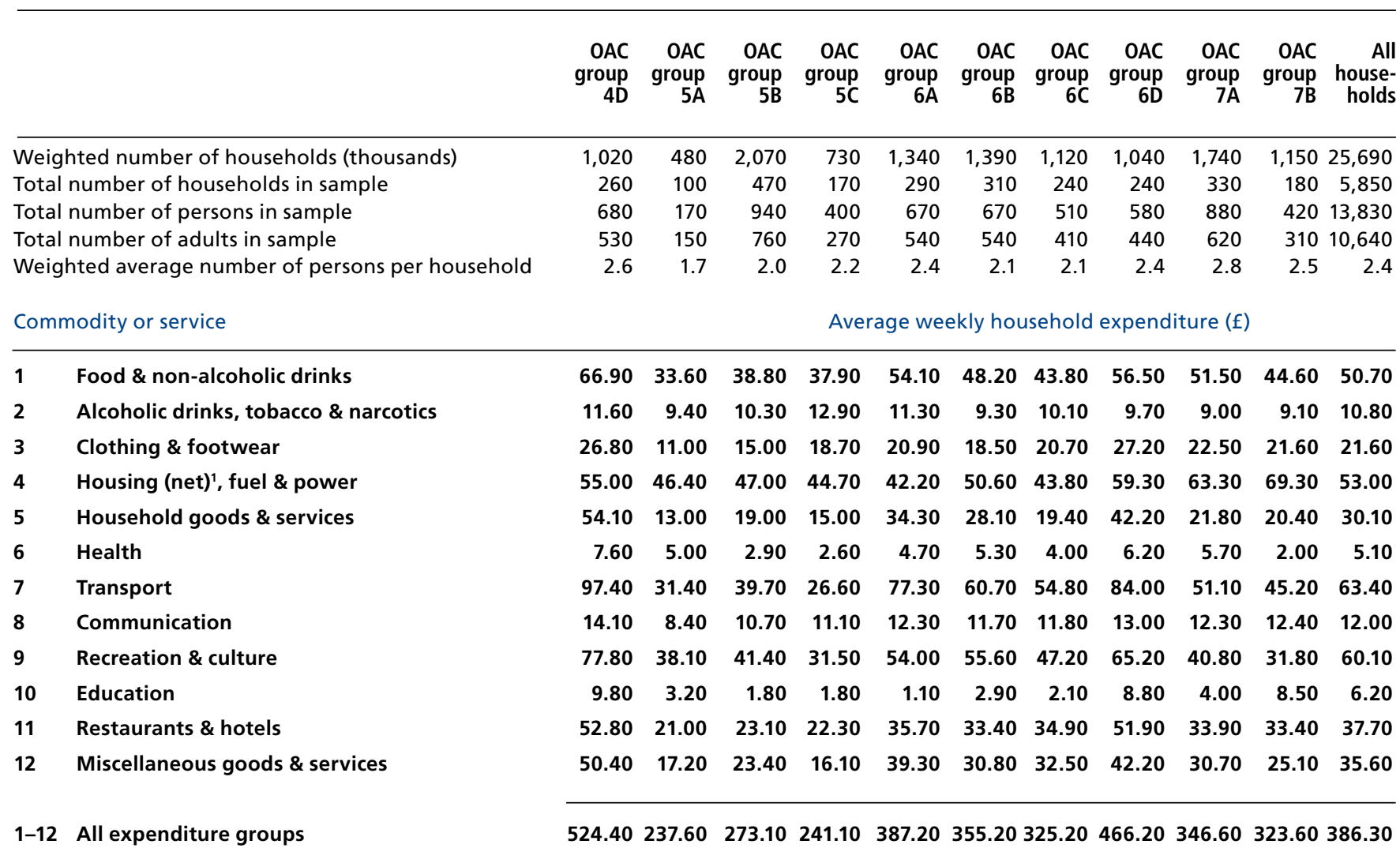

Note: Please see page xiii for symbols and conventions used in this report.

1 Excluding mortgage interest payments, council tax and Northern Ireland rates 
Table A58

Average gross normal weekly household income by OAC supergroup, 2008

based on weighted data

\begin{tabular}{|c|c|c|c|c|c|c|c|c|}
\hline & $\begin{array}{r}\text { OAC } \\
\text { Super- } \\
\text { group } \\
1\end{array}$ & $\begin{array}{r}\text { OAC } \\
\text { Super- } \\
\text { group } \\
2\end{array}$ & $\begin{array}{r}\text { OAC } \\
\text { Super- } \\
\text { group } \\
3\end{array}$ & $\begin{array}{r}\text { OAC } \\
\text { Super- } \\
\text { group } \\
4\end{array}$ & $\begin{array}{r}\text { OAC } \\
\text { Super- } \\
\text { group } \\
5\end{array}$ & $\begin{array}{r}\text { OAC } \\
\text { Super- } \\
\text { group } \\
6\end{array}$ & $\begin{array}{r}\text { OAC } \\
\text { Super- } \\
\text { group } \\
7\end{array}$ & $\begin{array}{r}\text { All } \\
\text { house- } \\
\text { holds }\end{array}$ \\
\hline Weighted number of households (thousands) & 4,180 & 1,520 & 3,450 & 5,480 & 3,290 & 4,890 & 2,890 & 25,690 \\
\hline Total number of households in sample & 990 & 290 & 880 & 1,360 & 740 & 1,080 & 500 & 5,850 \\
\hline Total number of persons in sample & 2,480 & 560 & 2,130 & 3,430 & 1,500 & 2,430 & 1,300 & 13,830 \\
\hline Total number of adults in sample & 1,800 & 480 & 1,650 & 2,680 & 1,170 & 1,930 & 930 & 10,640 \\
\hline Weighted average number of persons per household & 2.5 & 1.9 & 2.4 & 2.5 & 2.0 & 2.3 & 2.6 & 2.4 \\
\hline Gross normal weekly household income & 527.80 & 1226.70 & 819.70 & 899.60 & 413.90 & 683.60 & 620.90 & 713.10 \\
\hline
\end{tabular}

Note: Please see page xiii for symbols and conventions used in this report. 